DATA LIBFAPY \& APICHIVES Woods Hole Oceanographic Institution

271
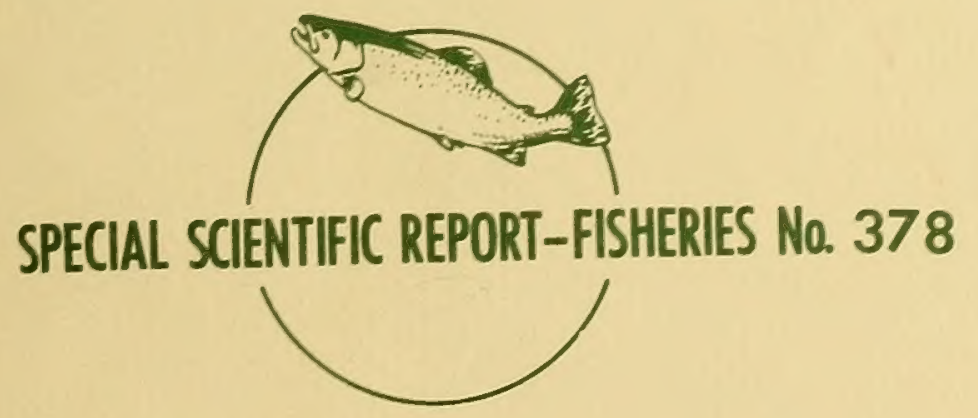

United States Department of the Interior, Stewart L. Udall, Secretary

Fish and Wildlife Service, Clarence F. Pautzke, Commissioner

Bureau of Commercial Fisheries, Donald L. McKernan, Director

\section{DRIFT BOTTLE RECORDS FOR THE GULF OF MAINE, GEORGES BANK AND BAY OF FUNDY, 1956-58}

by

Dean F. Bumpus

Contribution No. 1146 from the Woods Hole Oceanographic Institution

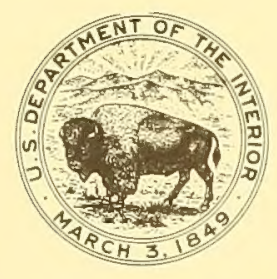

United States Fish and Wildlife Service

Special Scientific Report--Fisheries No. 378

Washington, D. C. May 1961 
This work was financed by the Bureau of Commercial Fisheries under Contract No. 14-17-008-62, with funds made available under the Act of July 1, 1954 (68 Stat. 376), commonly known as the SaltonstallKennedy Act. 


\title{
DRIFT BOTTLE RECORDS FOR THE GULF OF MAINE, GEORGES BANK AND BAY OF FUNDY, 1956-58
}

\author{
by
}

Dean F. Bumpus

The drift-bottle data tabulated here are the result of extended studies by the United States Fish and Wildlife Service, the Fisheries Research Board of Canada, and the Woods Hole Oceanographic Institution in the Gulf of Maine area.

Since the previous data report, Bumpus and Day (1957), drift bottles have not only been released during voyages of research vessels in the area but also from Lightships and the U.S. Air Force Texas Towers. One set of data, Crawford 18, represents bottles released in Raleigh Bay off North Carolina. A number of bottles launched prior to the autumn of 1959 have been reported since the above-mentioned report went to press. These are included as an appendix as is one return from $T . N_{\bullet}$ Gill Cruise 8 , supplementary to those reported by Anderson and Gehringer (1959).

Some of the results of these and previous data are reported by Bumpus (1960). The several thousand drift bottles released during 1957 and 1958 , as part of the Passamaquoddy Fisheries Investigations, are not included here. The results of that study are found in Chevrier and Trites (1960).

The drift bottle paths, drawn on the accompanying figures, were deduced in a manner similar to that described by

Note.--Dean F. Bumpus, Oceanographer, Woods Hole Oceanographic Institution, Woods Hole, Mass.
Day (1958). Briefly, the final paths drawn on each chart resulted from constructing a consistent field of motion for any given set of data, commencing with th shortest drifts in both time and distance and continuing until all paths had been drawn.

\section{Bottle Type and Distribution}

The bottles used were those commonly employed for carbonated drinks. They were of clear glass and measured approximately $81 / 2$ inches high and $21 / 4$ inches in diameter with a capacity of 8 fluid ounces. Dry sand ballast was added to the majority of the bottles with the intention that they should float vertically at or near the surface and thus be less influenced by the action of winds and breaking waves. Where bottles were not ballasted, it is so indicated.

\section{The Data}

The original data are on file at the Woods Hole Oceanographic Institution, where a punch card system is maintained and supplemented as new returns are received and further studies initiated.

The listings below include all $\mathrm{re}-$ coveries on record as of August 31, 1960, from the following cruises and static locations. Diagrams of the surface circulation as deduced from the recoveries of the drift bottles launched on these cruises accompany the data. 
Silver Bay

Silver Bay

Harengus

Silver Bay

Harengus

19427 Delaware

Albatross III

Albatross III

Albatross III

Albatross III

Albatross III

Albatross III

Harengus

Harengus

Albatross III

Albatross III

Harengus

Albatross III

Delaware

1958 Albatross III

Delaware

Sackville

Sackville

Metacomet

Albatross III

Metacomet

Crawford

Albatross III

Sackville
Cruise AH5

\section{1}

2

AH6

4

AH7

57-1

88

90

92

93

94

95

17

18

104

105

106

$57-9$

107

$57-9(111)$

37

38

$58-1$

110

58-2

18

111

39
Sept. 19-Oct. 4

Sept. 21-Oct. 5

Figure 1

Oct. 15-Oct. 25

Oct. 16-Oct. 28

Figure 2

Nov, 14-Nov. 30

Nov. 14-Dec. 1

Jan. 26-Feb. 4

Feb. 19-Feb. 28

Figure 4

Apr. 11-Apr. 17 Figure 5

Apr. 25-May 1 Figure 6

May 8-May $16 \quad$ Figure 7

May 22-May 28 Figure 8

June 5-June 9 Figure 9

Sept. 9-Sept. 15 Figure 10

Oct. 7 -Oct. 25

Oct. 9-Oct. 17

Figure 11

Oct. 29-Nov. 8

Figure 12

Nov. 5-Nov. 21

Der 3-Dec. 19

Dec. 17-Dec. 19

Figure 13

Jan. 18-Jan. 20

Jan. 28-Jan. 29

Figure 14

Feb. 6-Feb. 27

Figure 15

Apr. 4-Apr. 5

Apr. 8-Apr. 26

Figure 16

May 6-May 7

May 8-May 11

Figure 17

May 17 -June 3

Figure 18

May 20-May 24

May 22-May 27

Figure 19 
Sept. 9-Sept. 15

Dartmouth

Albatross III

118

Delaware

Albatross III

Harengus

Albatross III

Harengus

Delaware

Sambro Lightship

Lurcher Lightship

Princess Helene

Portland Lightship

Texas Tower \#2

Nantucket Lightship

Texas Tower \#4

\section{LITERATURE CITED}

ANDERSON, W. W., AND J. W. GEHRINGER.

1959. Physical, oceanographic, biological and chemical data, South Atlantic coast of the United States. M/V Theodore N. Gill Cruise 8. U.S. Fish and Wildlife Service, Special Scientific Report--Fisheries No. 303, 227 p.

BUMPUS, D. F., AND C. G. DAY.

1957. Drift bottle records for the Gulf of Maine and Georges Bank, 1931-56. U. S. Fish and Wildlife Service, Special Scientific Report--Fisheries No. 242, 64 p.
Sept. 9-Sept. 15

Figure 20

Sept. 23-Oct. 1

Oct. 6-Oct. 14

Oct. 8-Oct. 16

Figure 21

Oct. 15-Oct. 30

Oct. 21 -Oct. 28

Nov. 10-Nov. 24

Dec. 12,18 and 19

Figure 22

Oct. 9, 1958-Dec. 30, 1958

Oct. 28, 1956-Dec. 31, 1958

Dec. 18, 1958-Dec. 31, 1958

July 23, 1958-Dec. 31, 1958

Jan. 31, 1958-Dec. 31, 1958

July 28, 1958 -Dec . 27, 1958

Oct. 7, 1958-Dec. 31, 1958

BUMPUS, D. F.

1960. Sources of water contributed to the Bay of Fundy by surface circulation. J. Fish. Res. Bd. Canada, 17 (2): 181-197.

CHEVRIER, J. R., AND R. W. TRITES. 1960. Drift bottle experiments in the Quoddy region, Bay of Fundy. J. Fish. Res. Bd. Canada, 17 (6): 743-762.

DAY, C. G.

1958. Surface circulation in the Gulf of Maine as deduced from drift bottles. U. S. Fish and Wildlife Service, Fishery Bulletin, vol. 58, no. 141 , p. 443-472. 


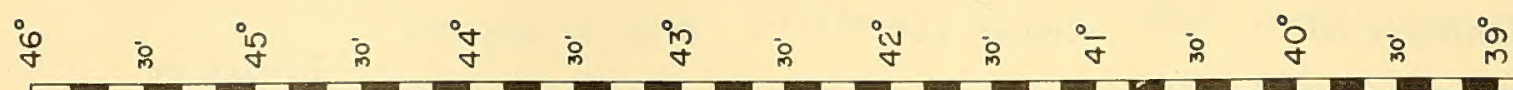

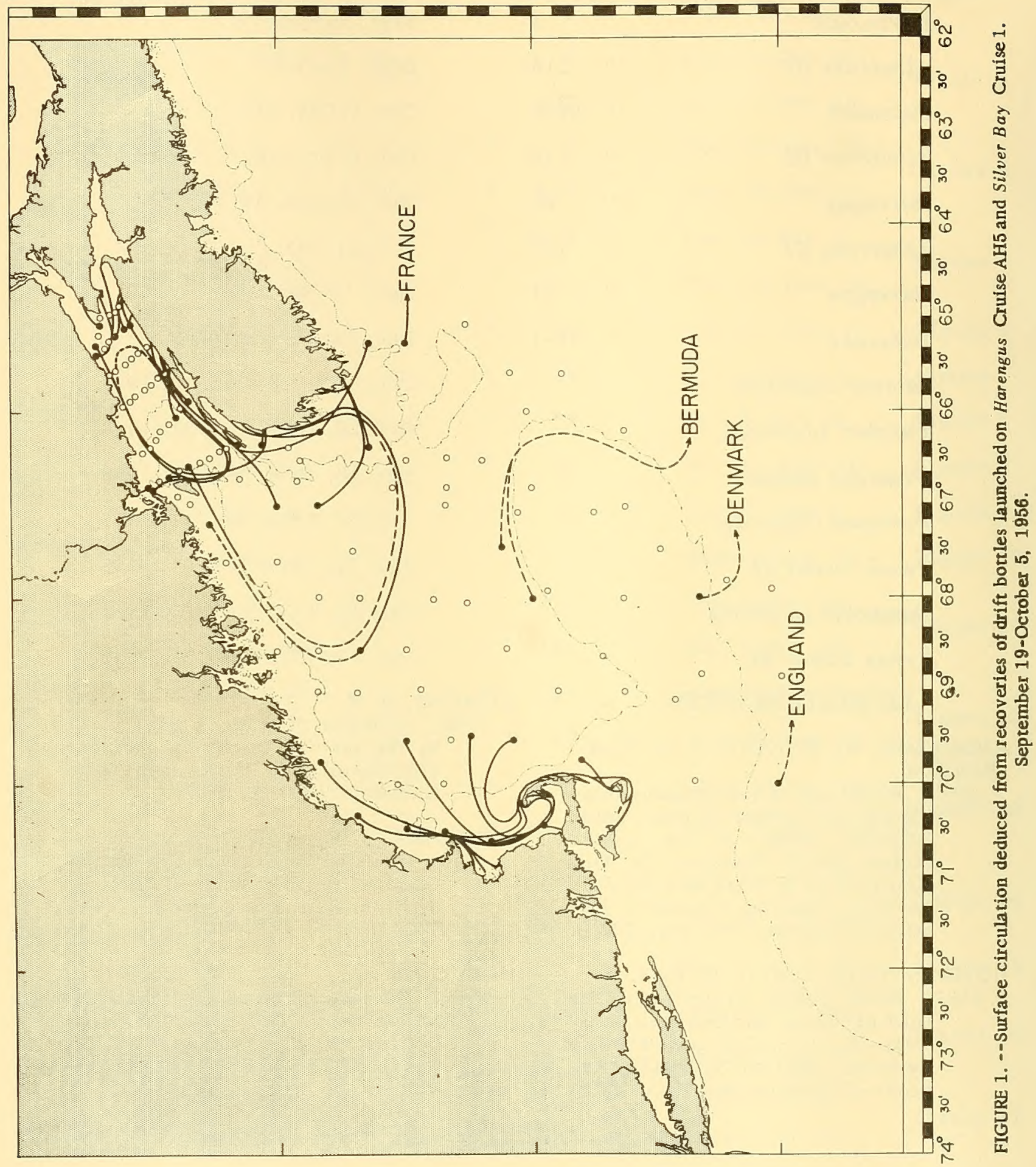




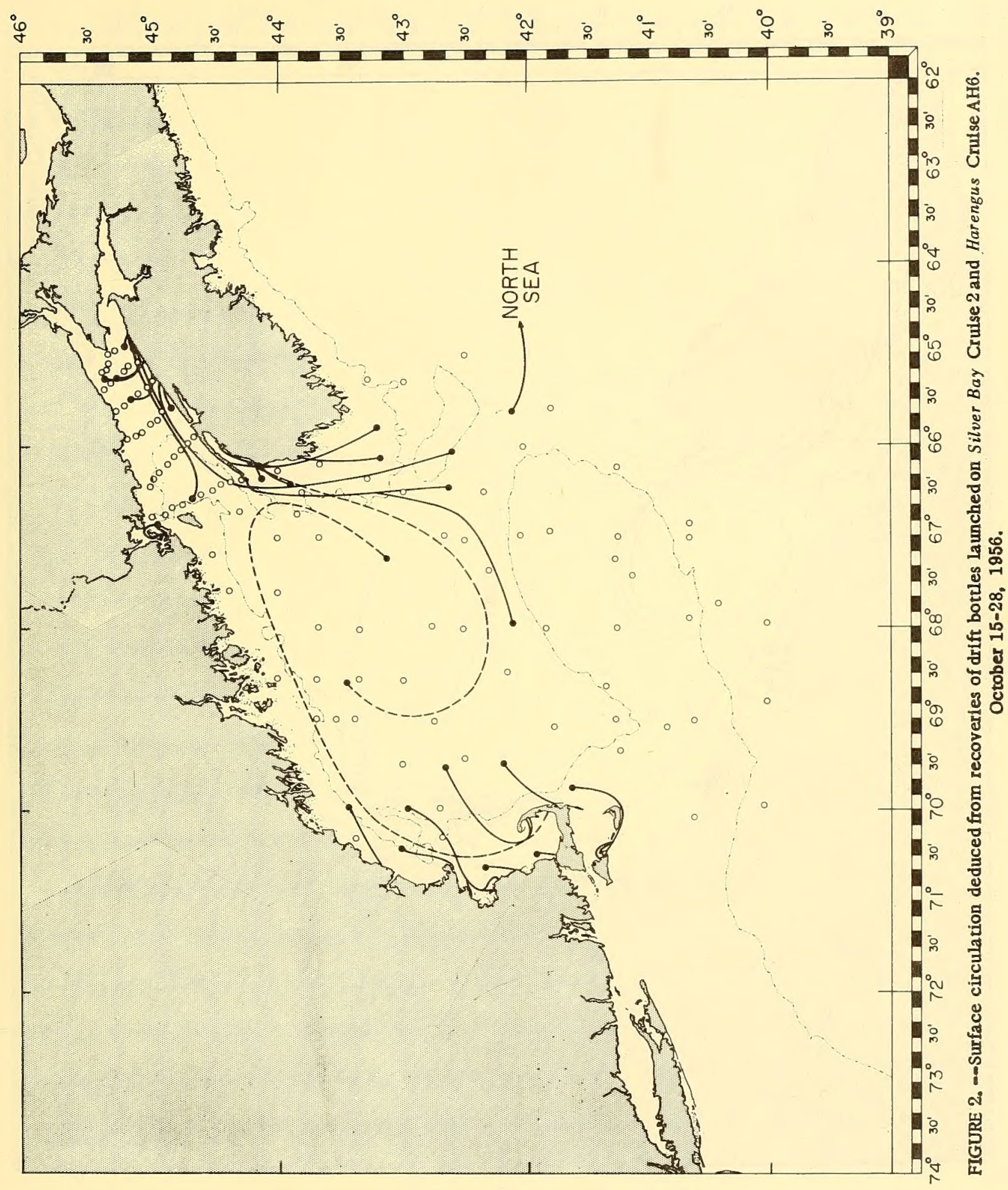




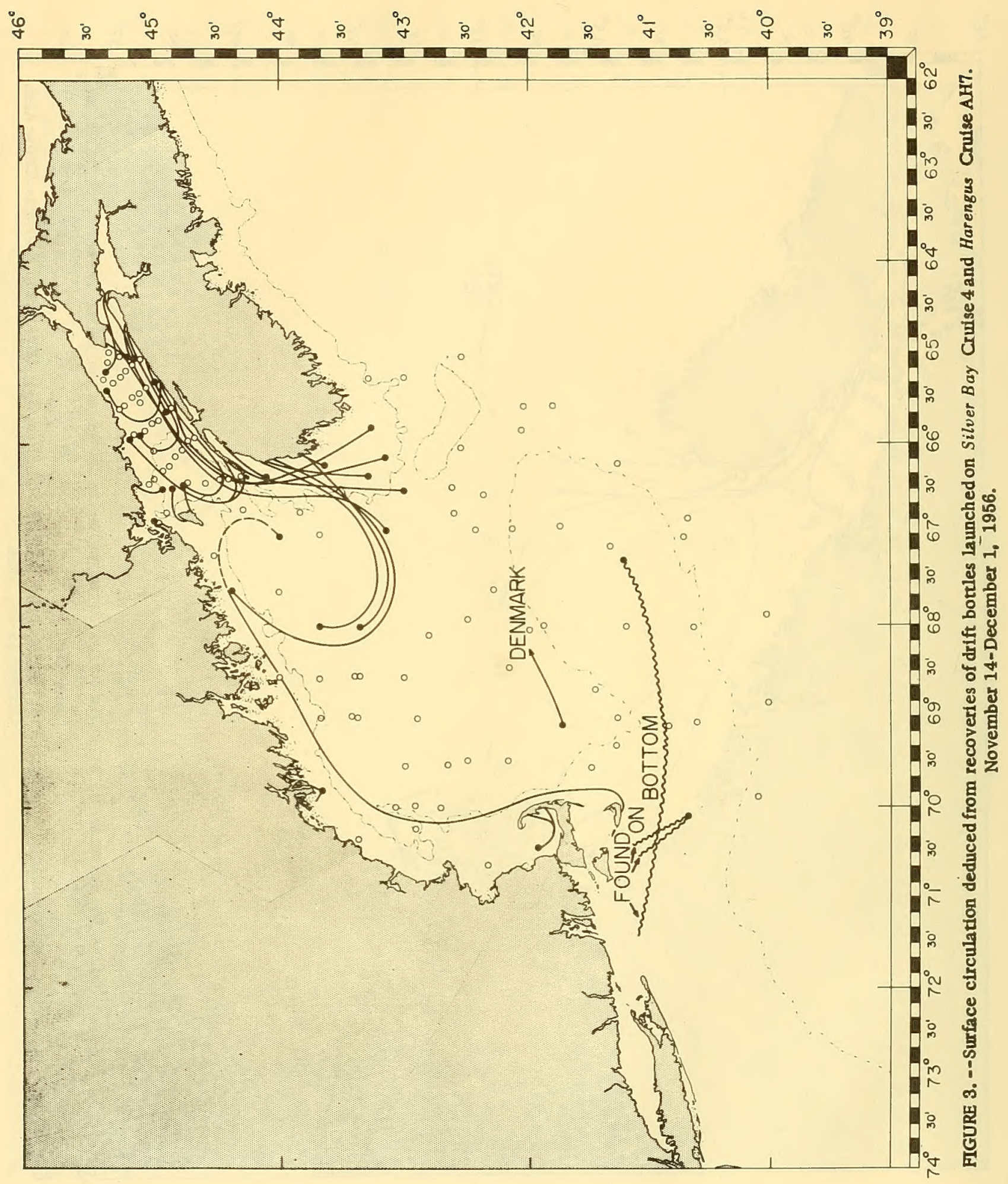




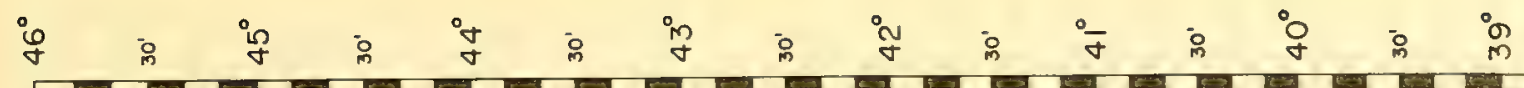

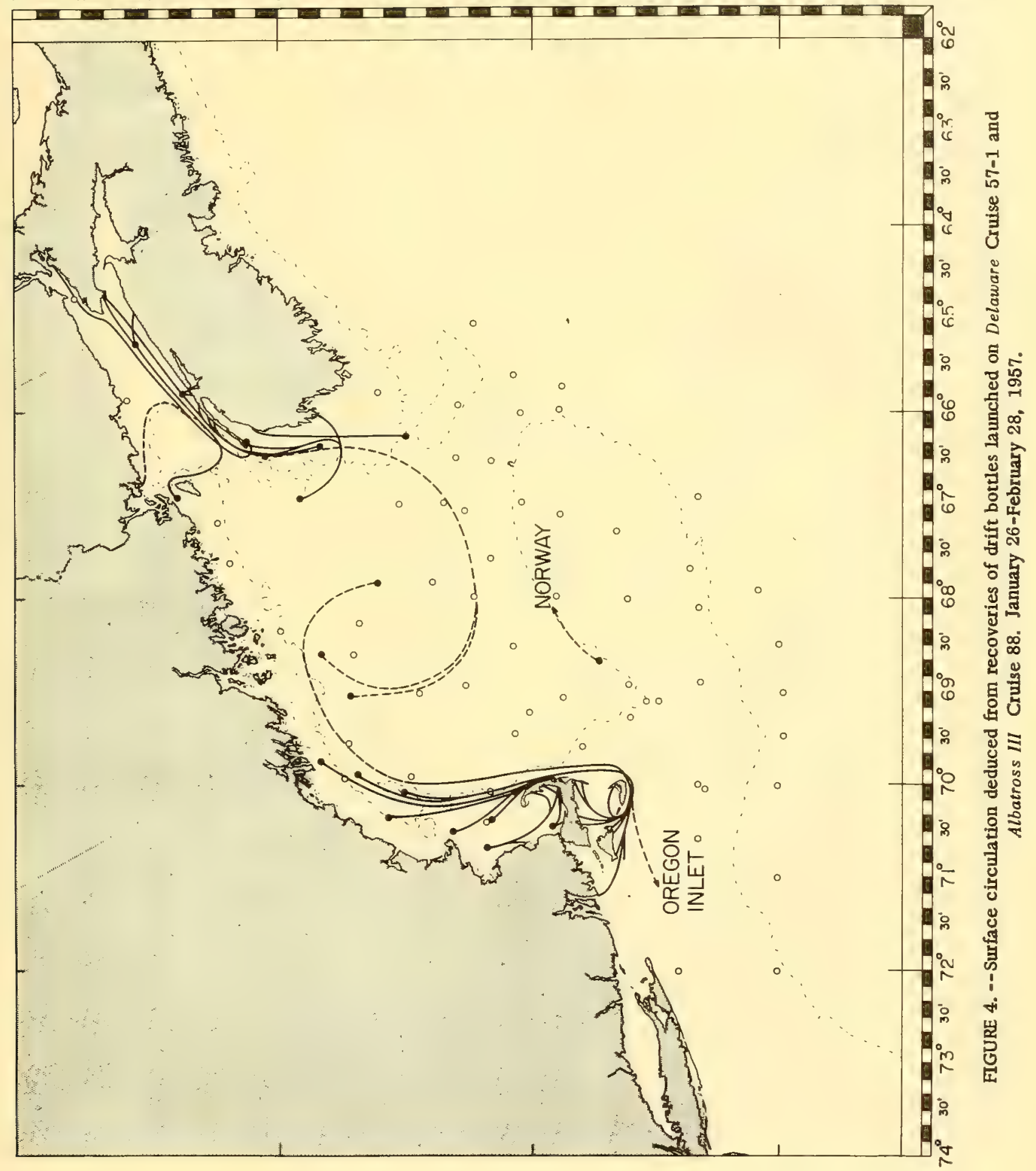



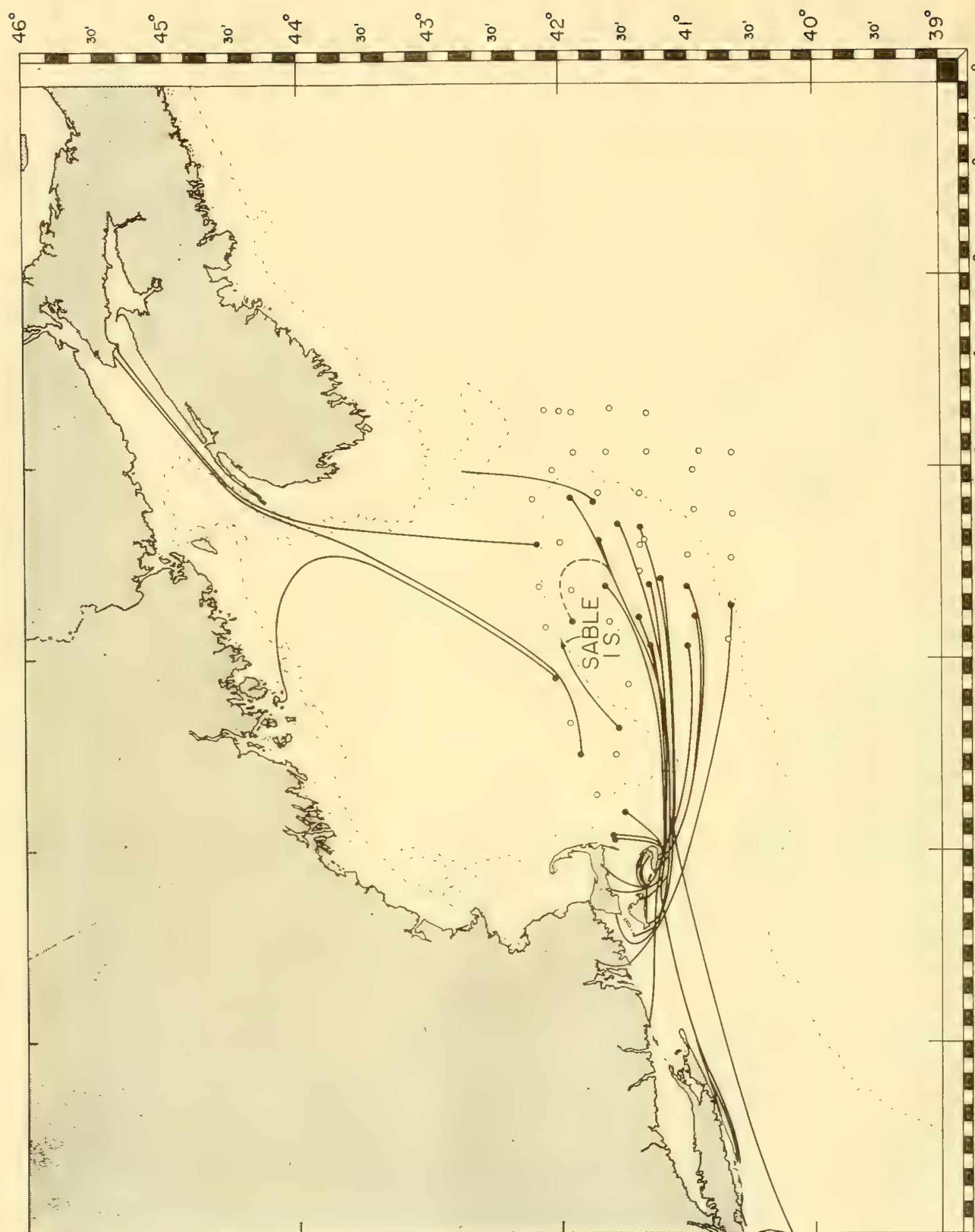


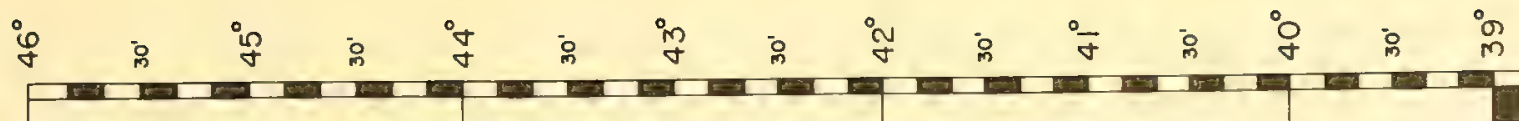

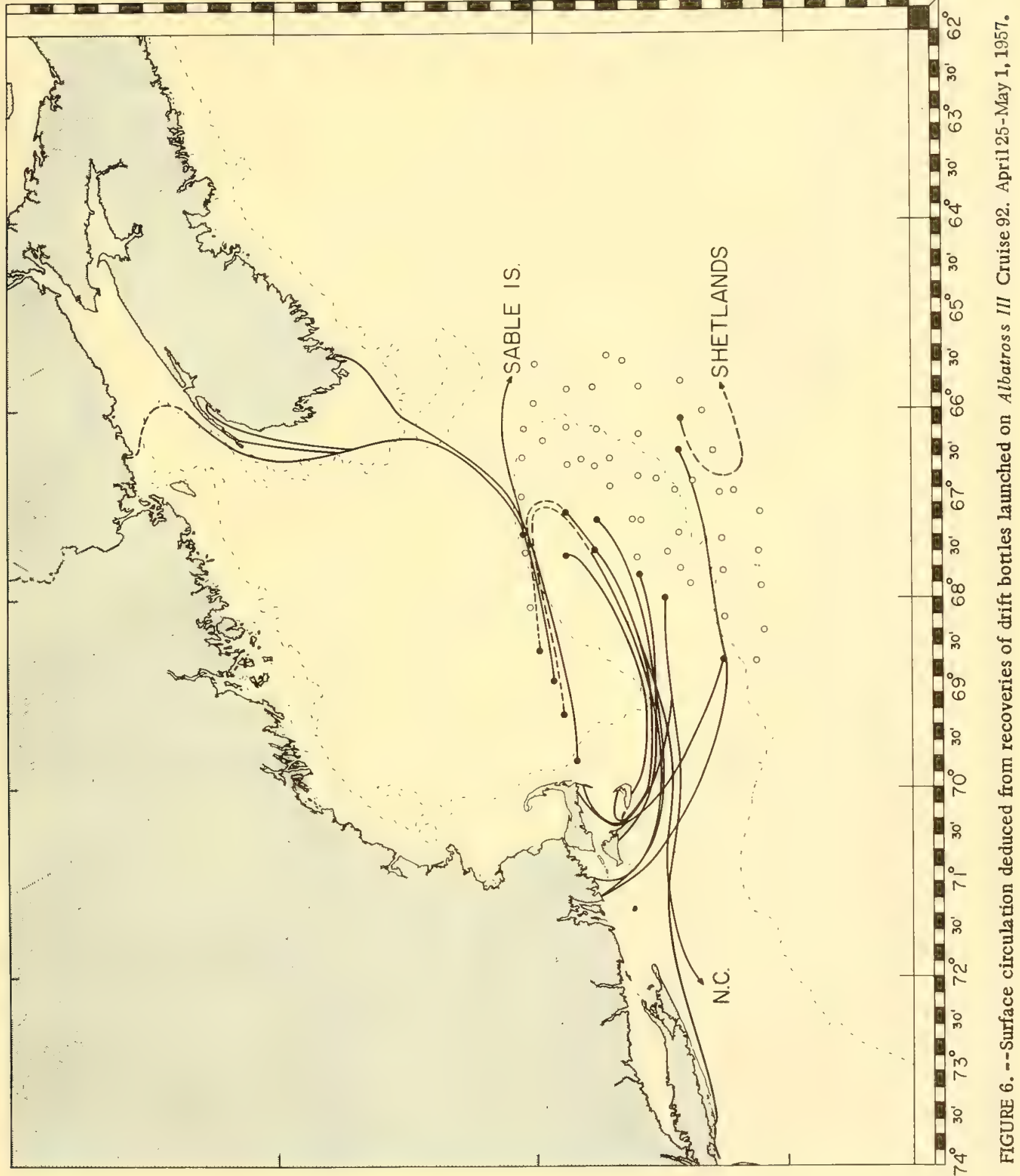









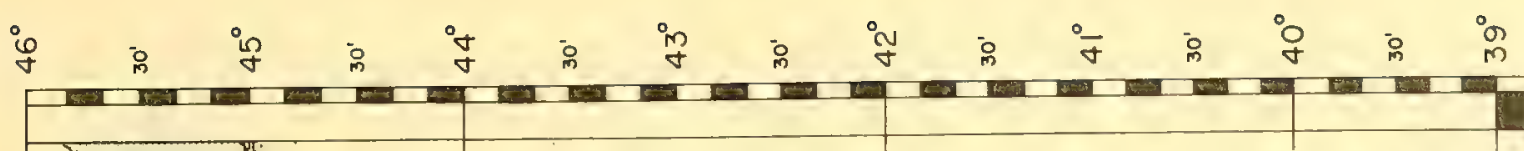

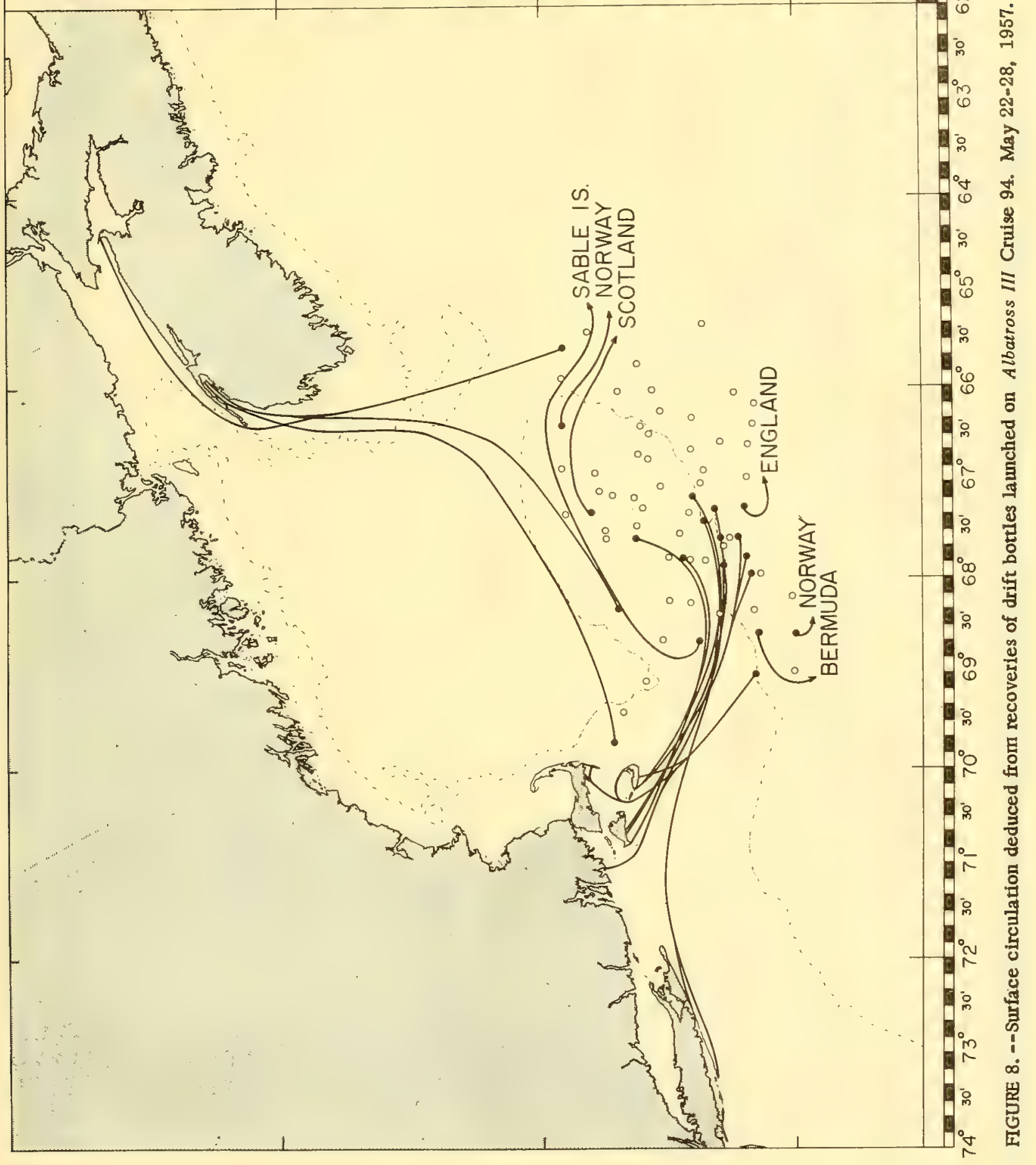




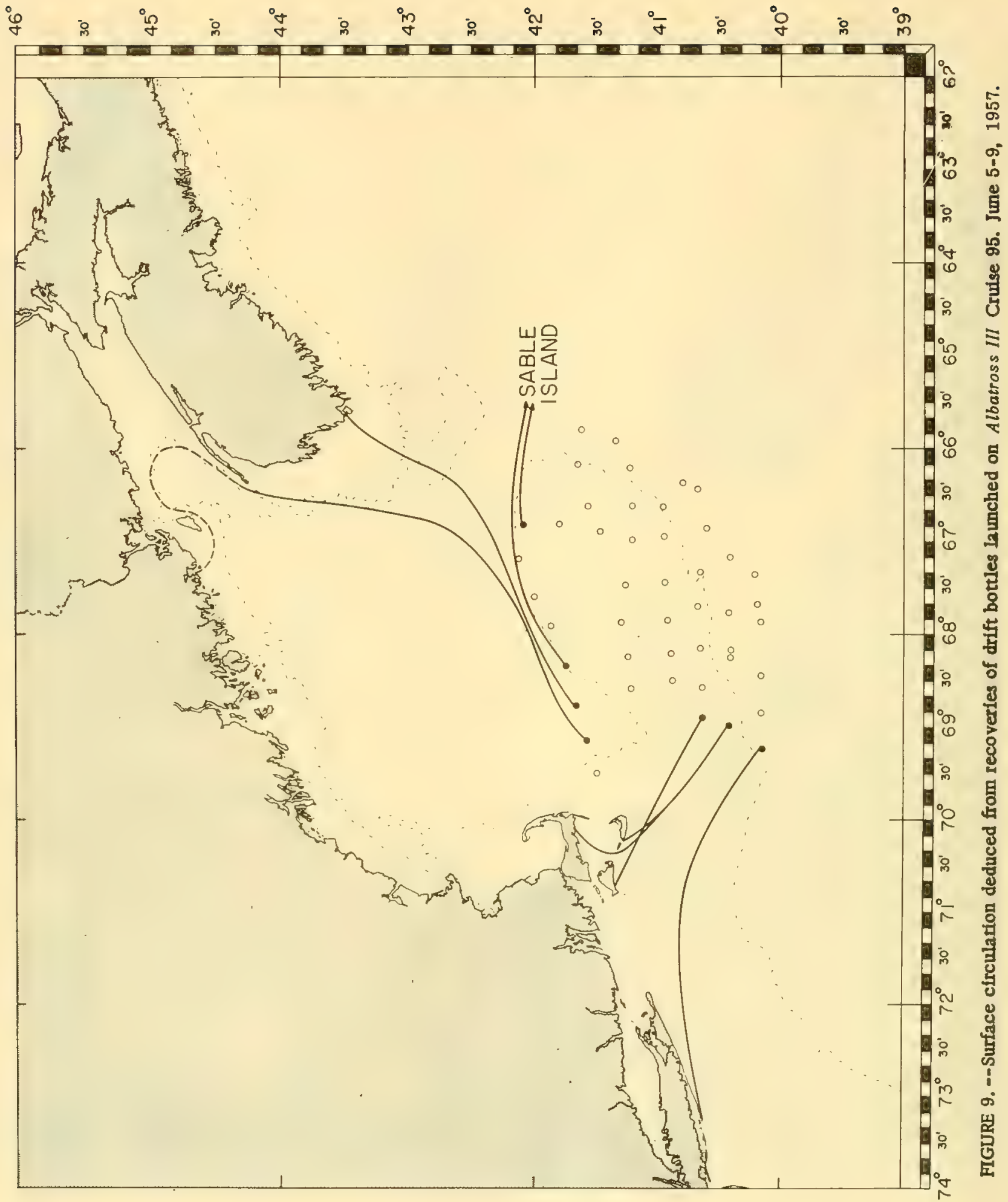




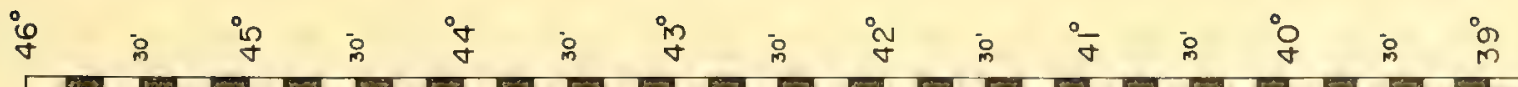

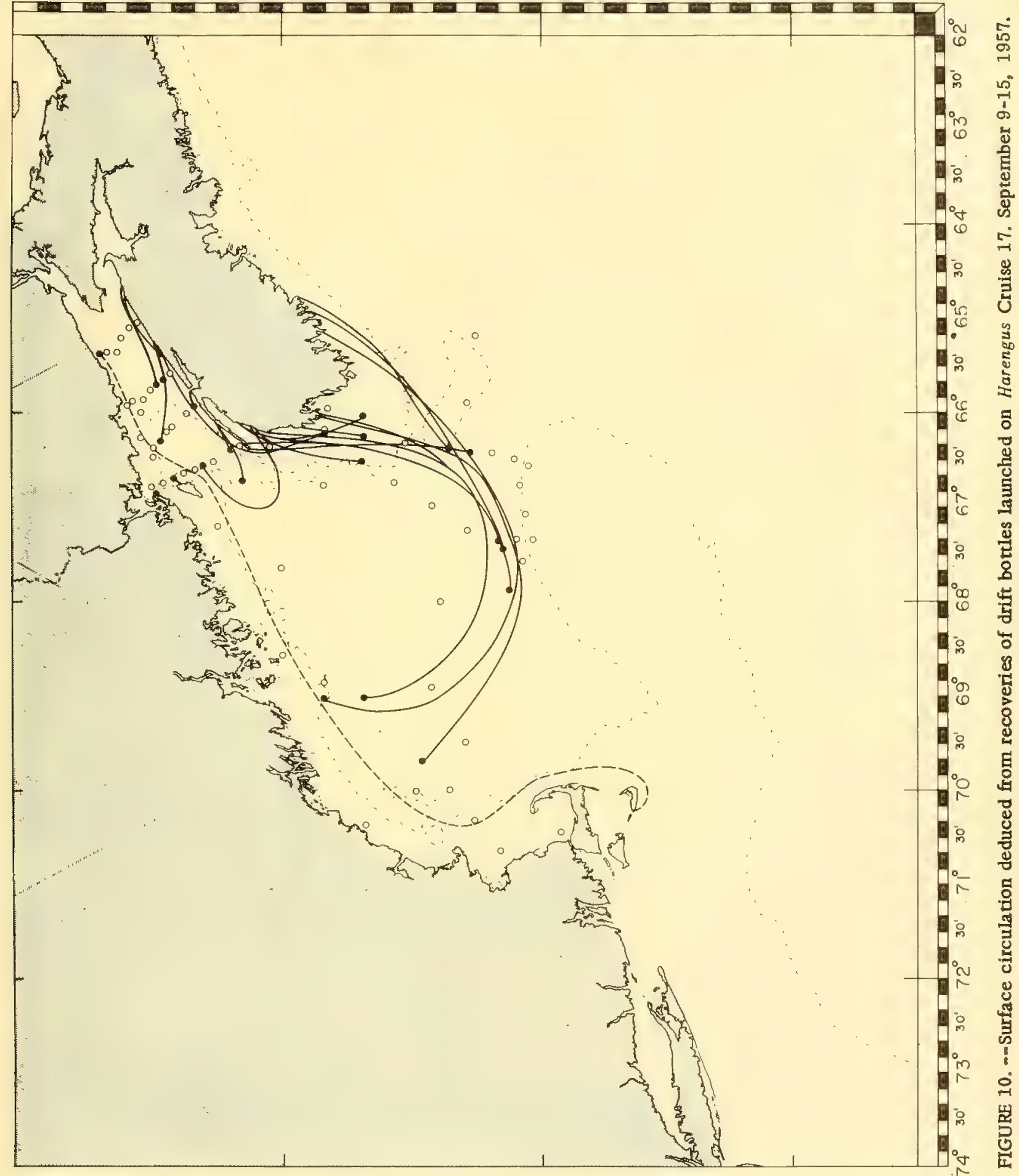






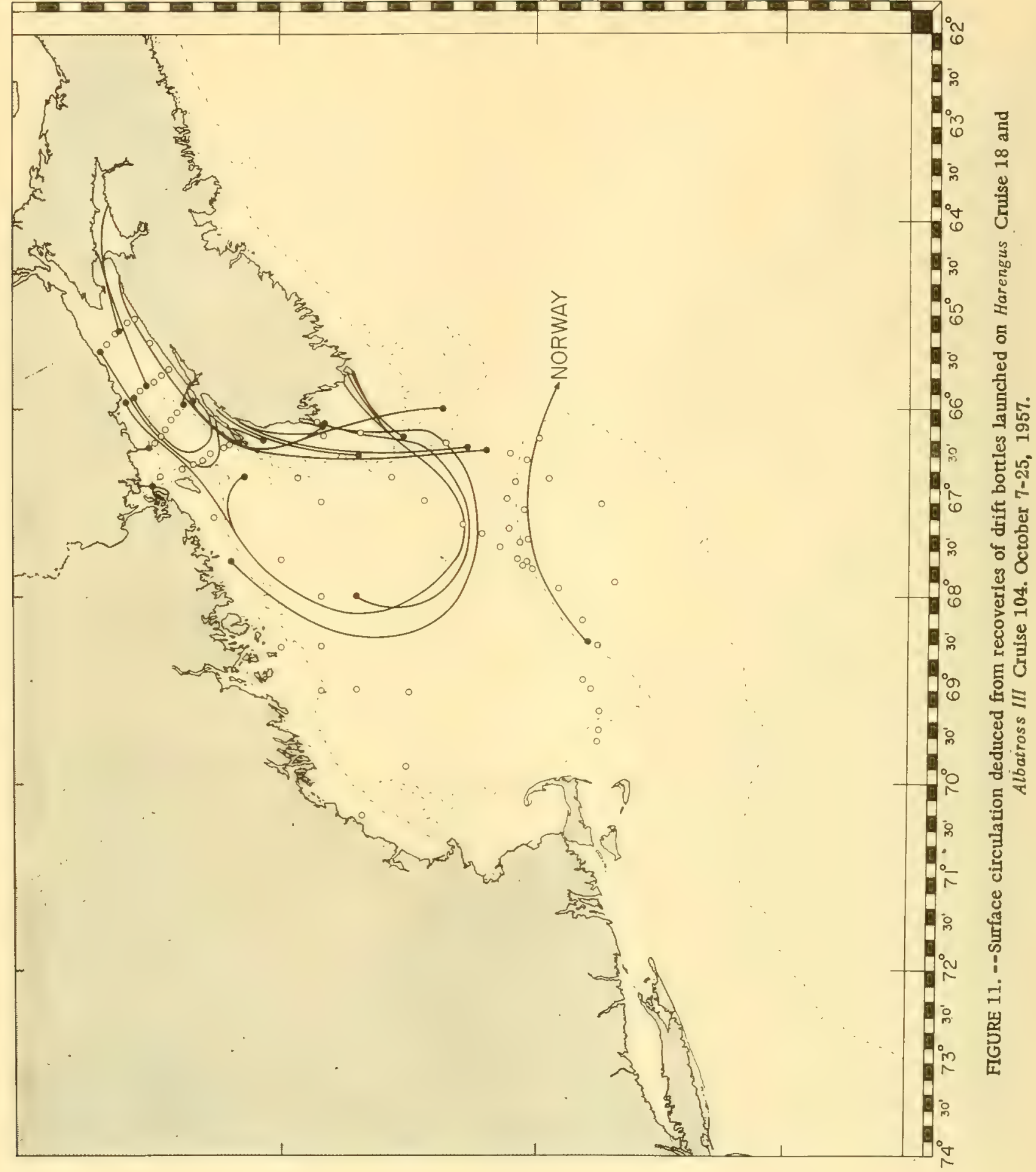




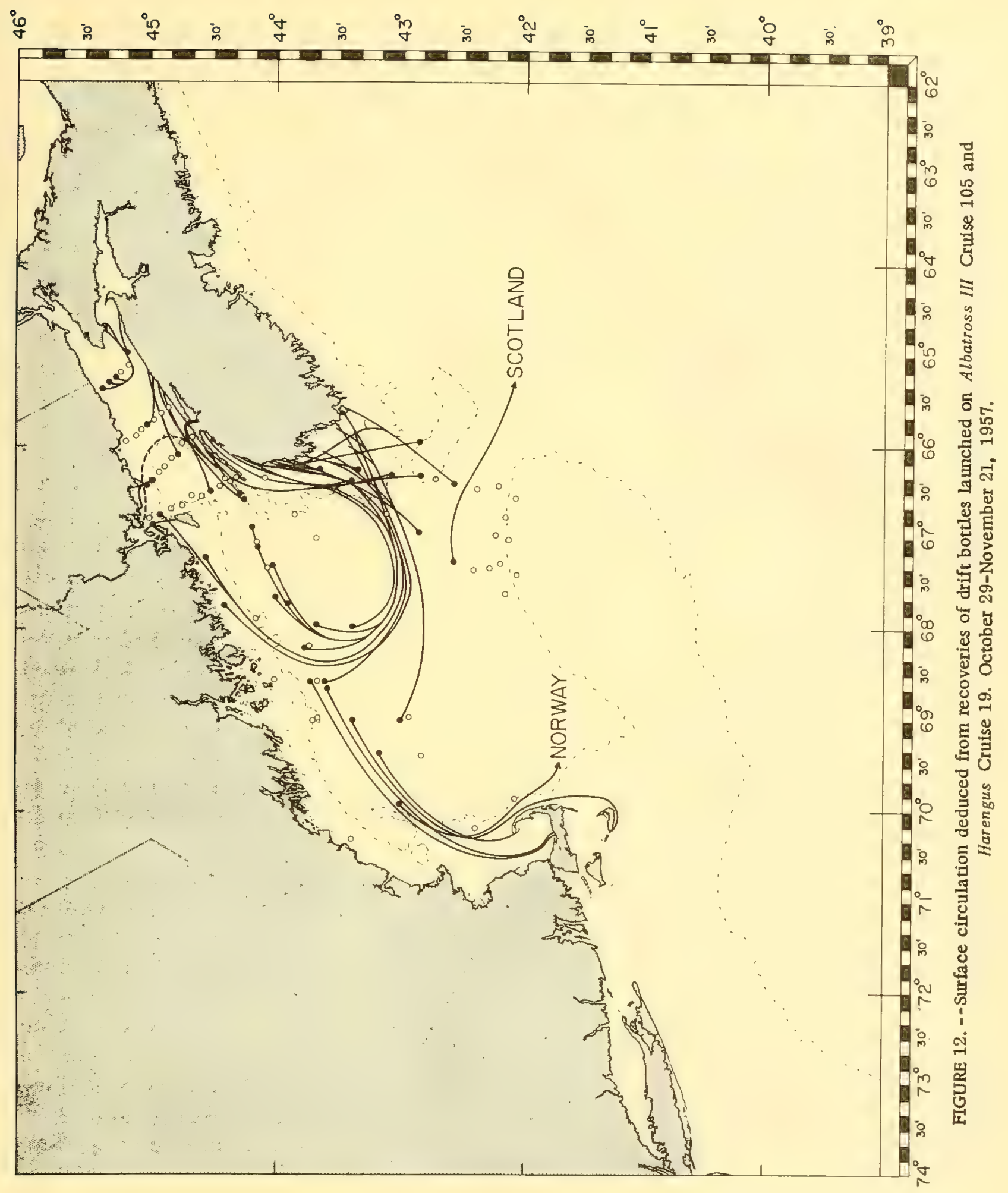









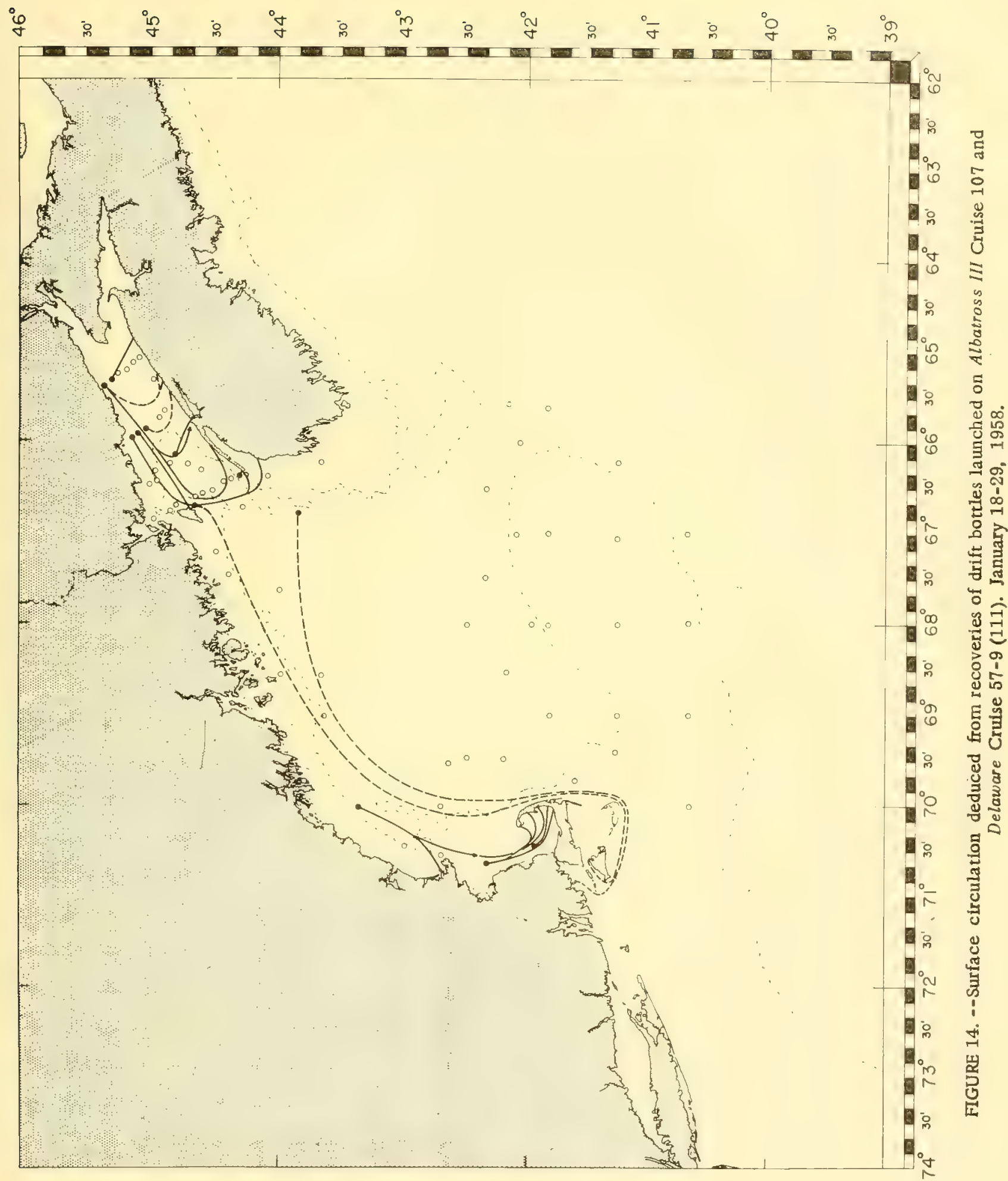




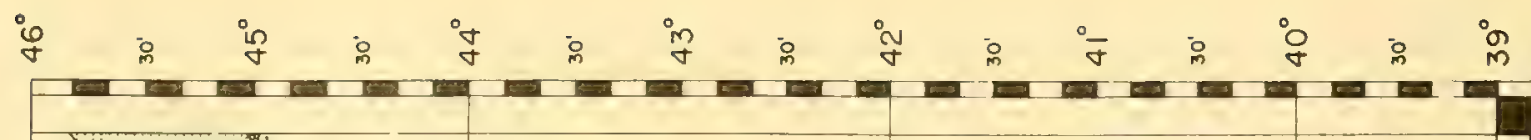

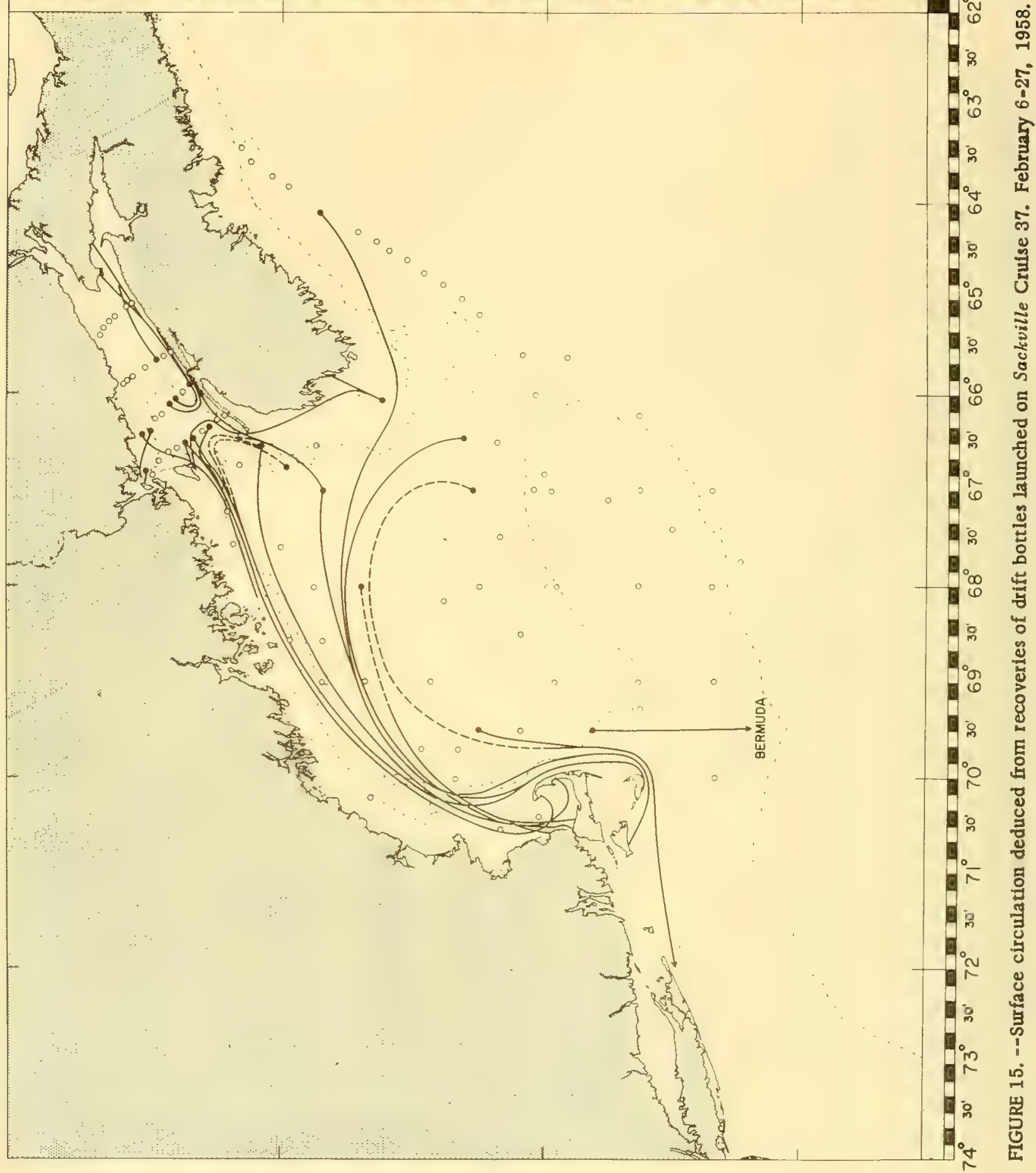




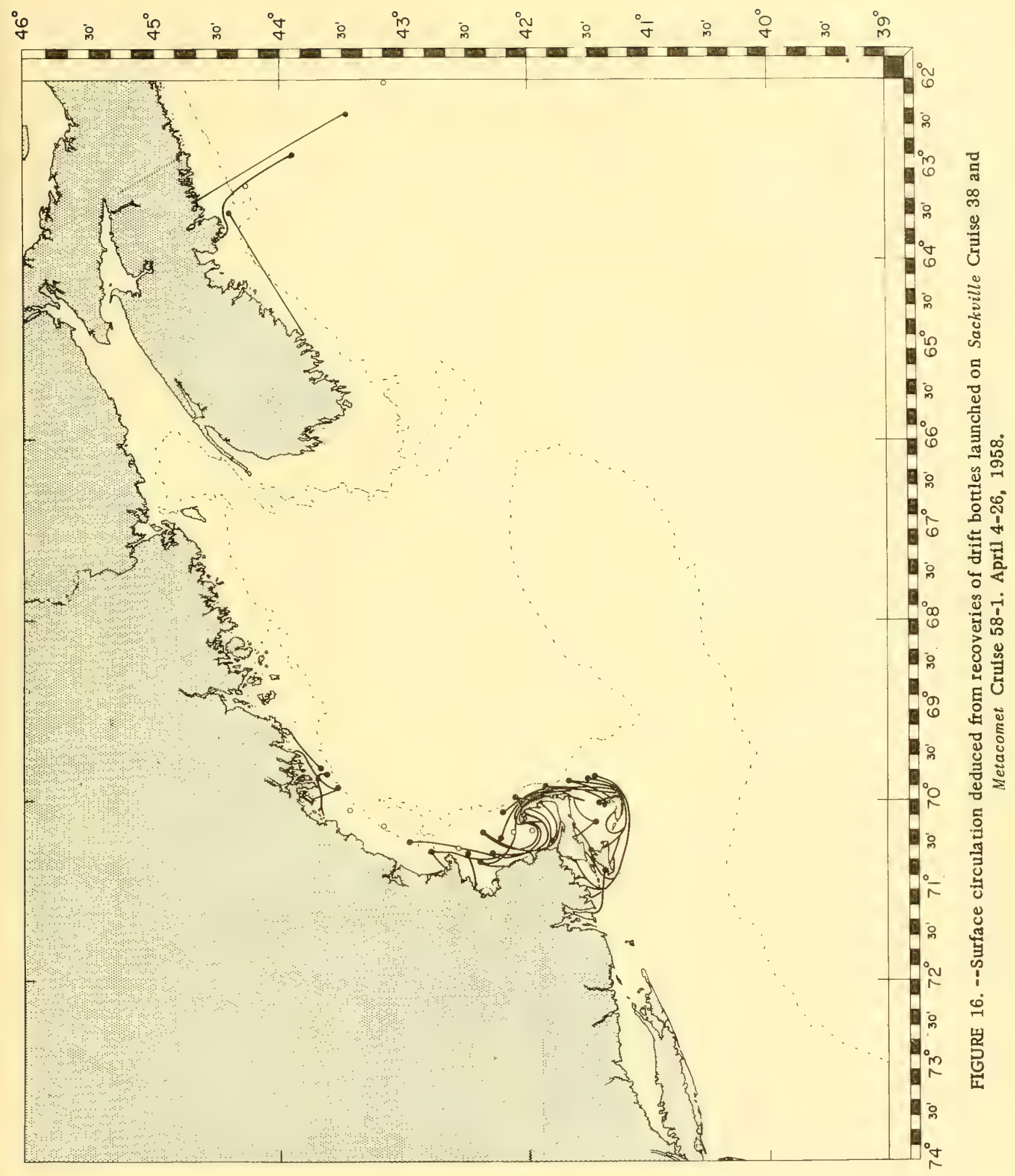




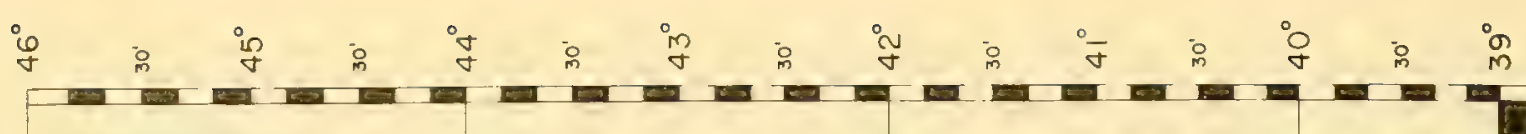

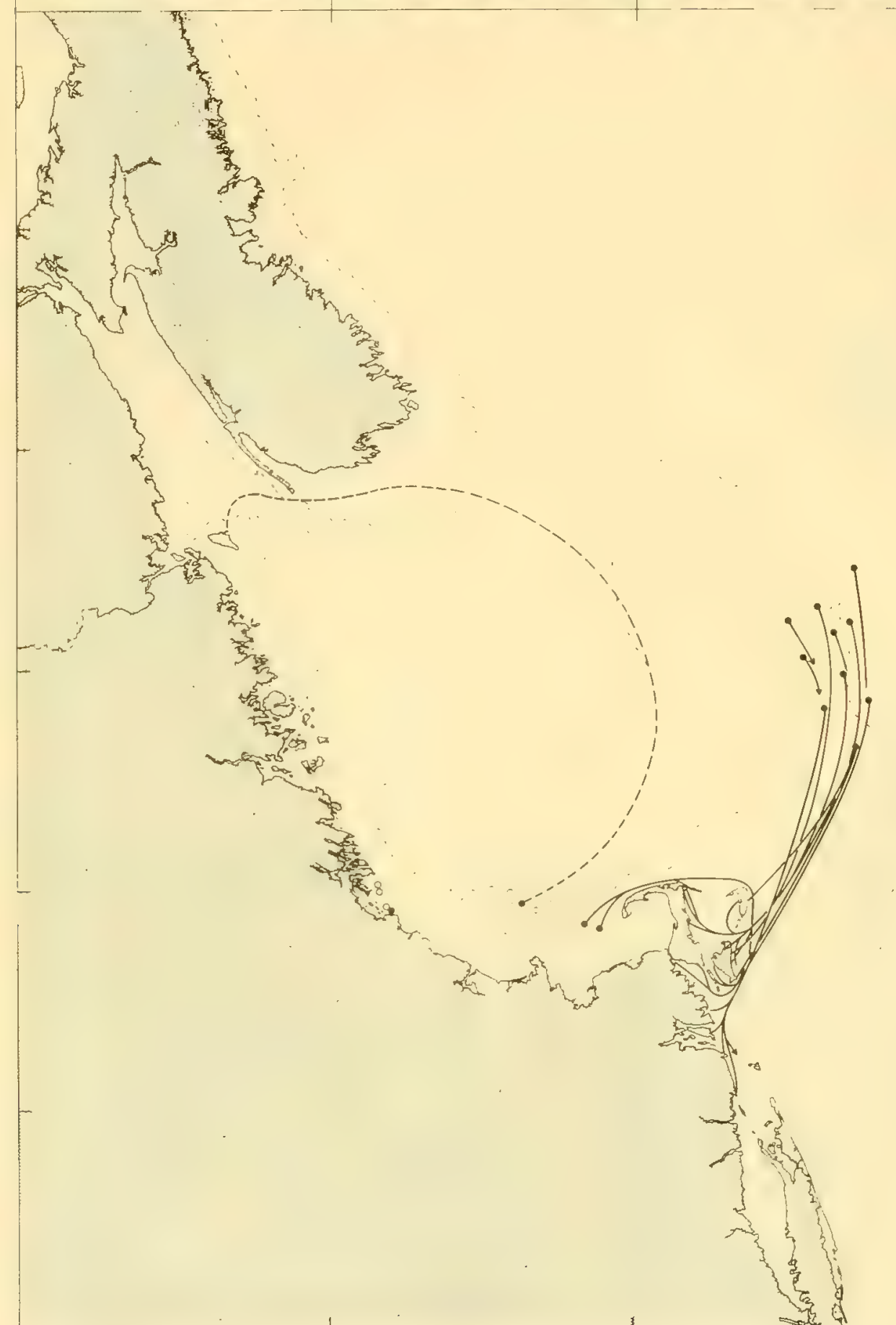

这

$a_{\text {in }} \overbrace{\tilde{T}}$

1 6 응

0.

日. 竞

10

a.

1.

1. 0

음

A. चु

1. 6 (

- 홍

Q D.

- 6 :

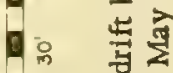

日.

10 0 品

日.



di

밍ㅎㅁ

d.

田 总

is

日.

a

回

月.

经总

这

告 


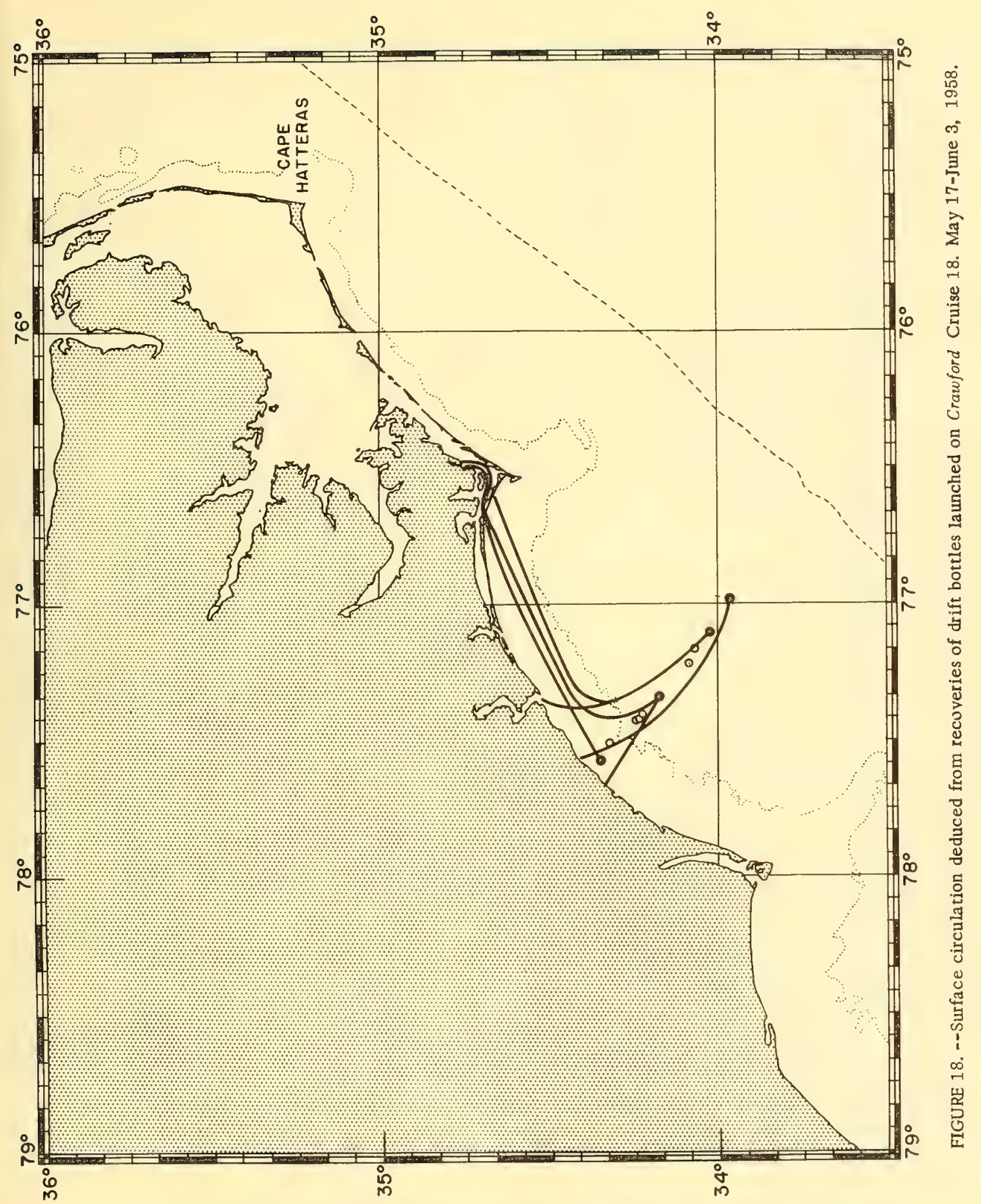




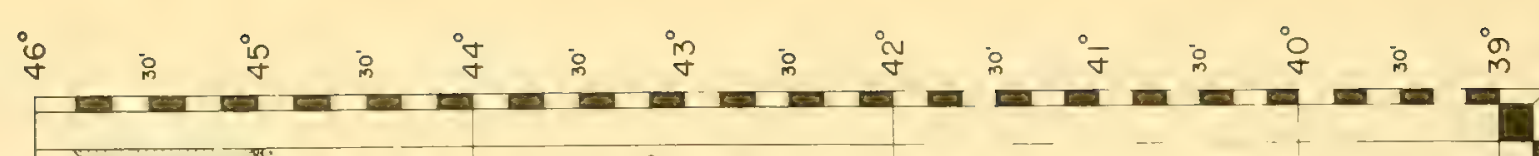

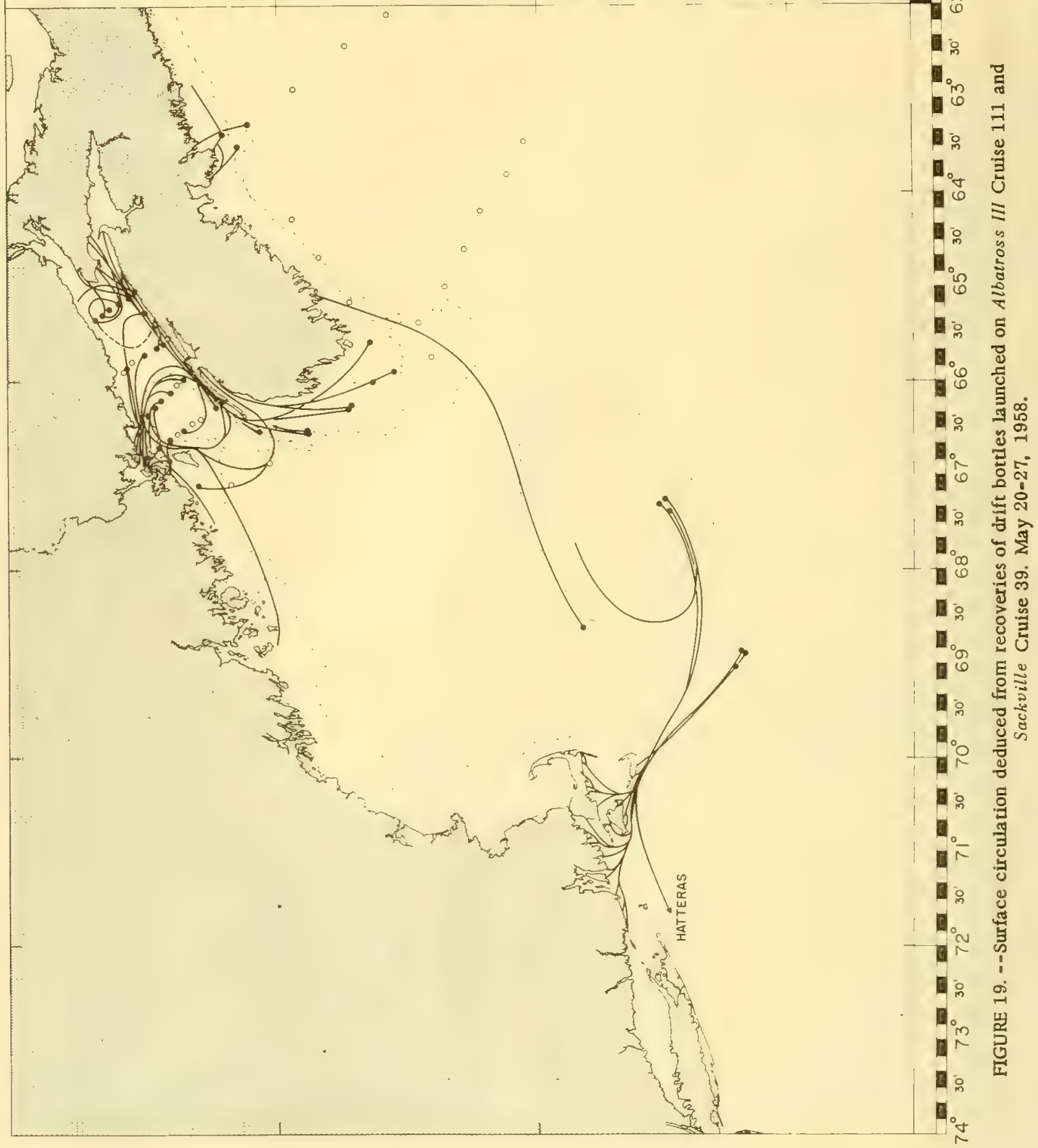




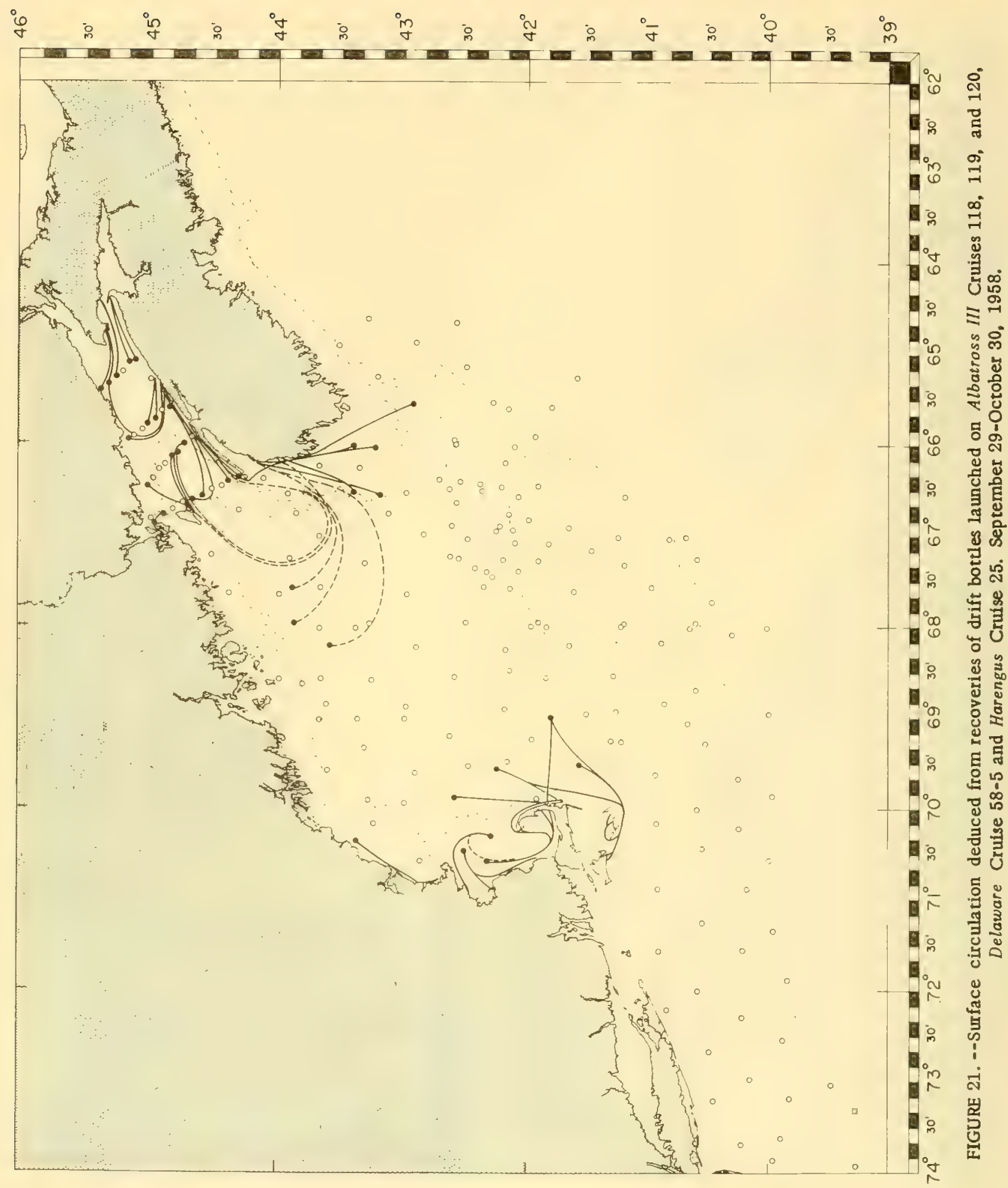




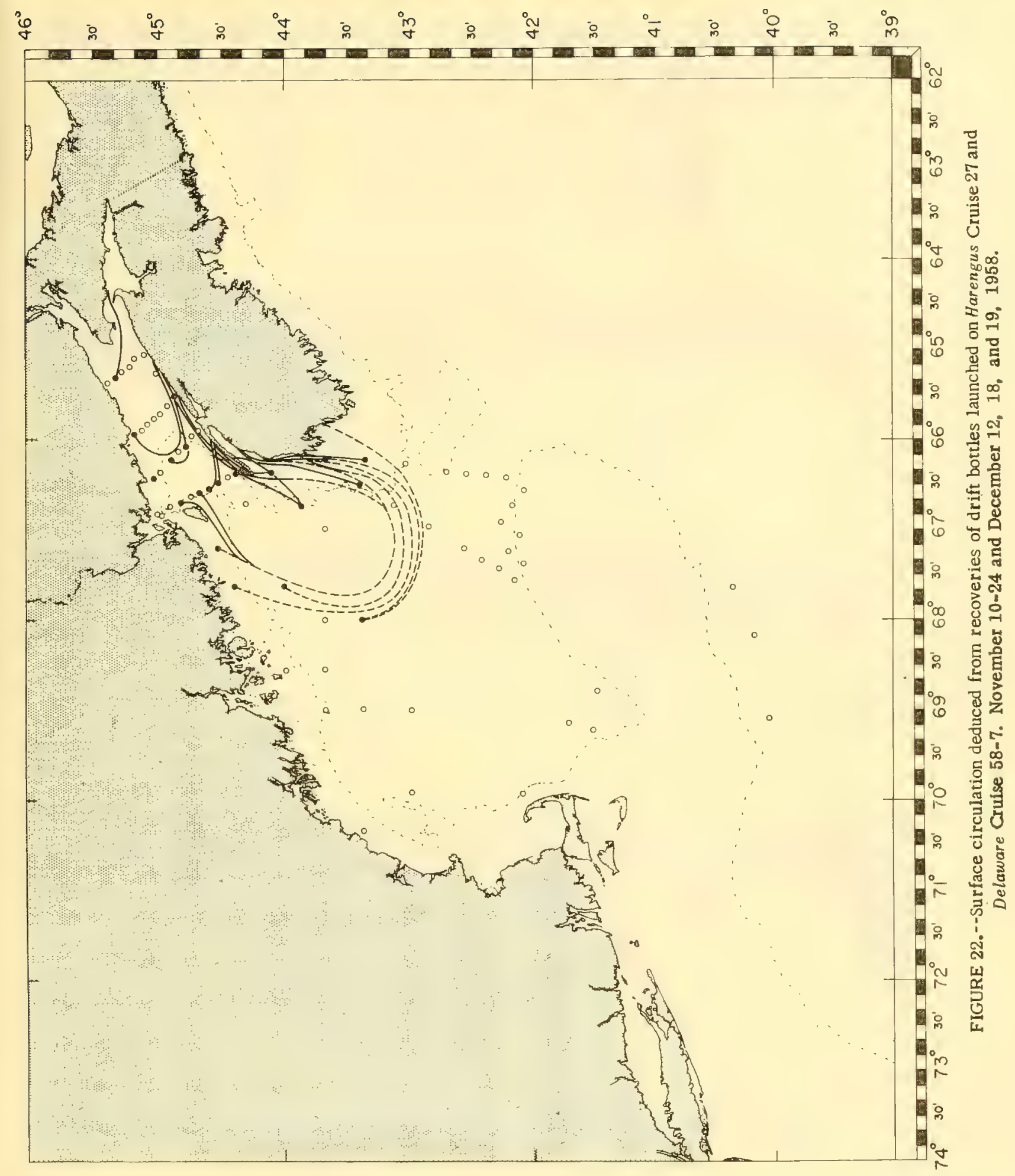




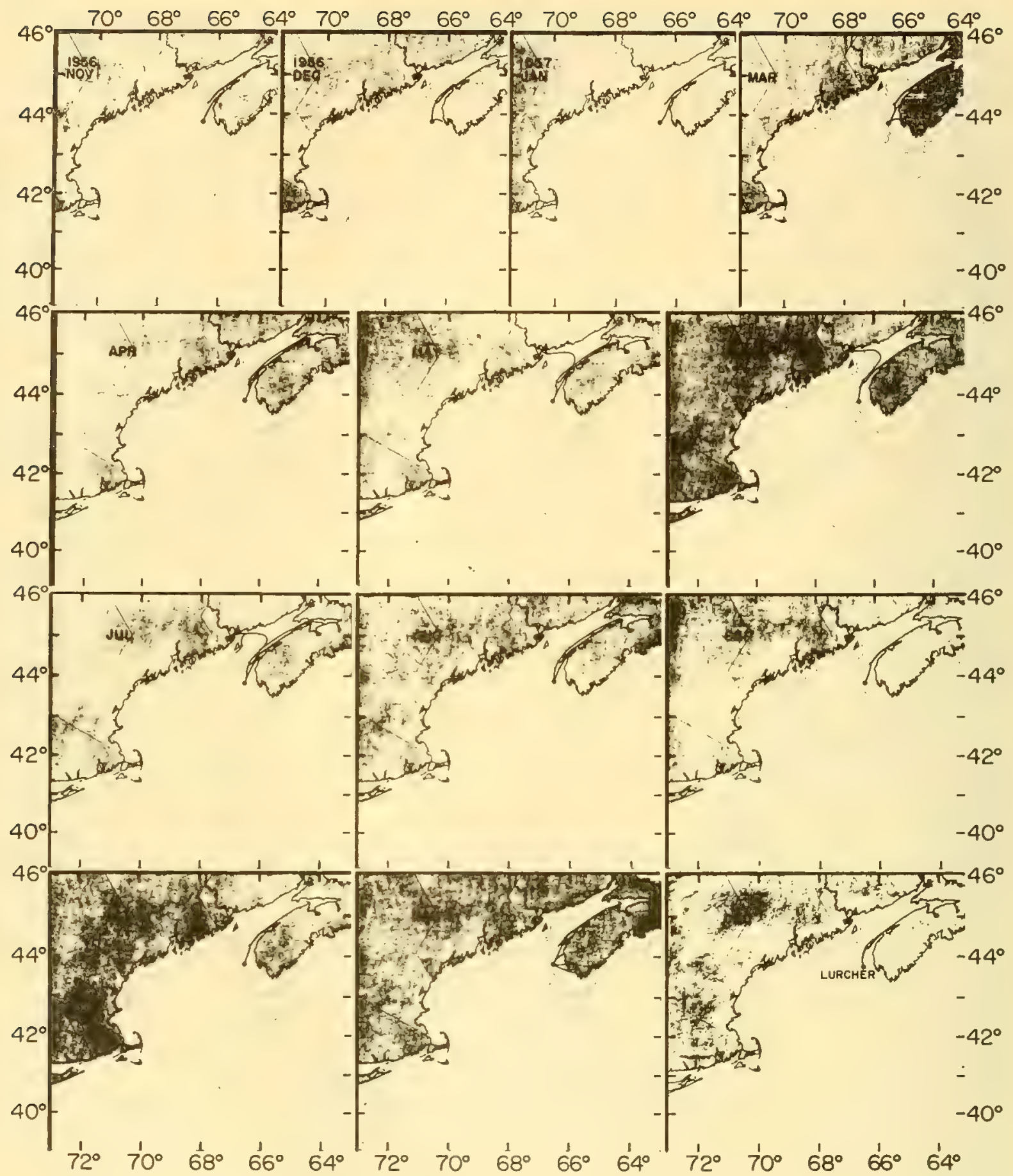

FIGURE 23.--Surface circulation deduced from recoveries of drift bottles launched from Lurcher Lightship each day during the period October 9, 1956-December 31, 1957. 




FIGURE 24.--Surface circulation deduced from recoveries of drift bottles launched from several positions each day during 1958. 


\section{Harengus Cruise AH5}

\section{September - 4 October}

1956

Stations 1-35, 67-74, et seq. , 6 bottles dropped, all ballasted.

Stations 36-66, 12 bottles dropped, even numbered bottles ballasted, odd numbered bottles not ballasted.

Released

\begin{tabular}{|c|c|c|c|c|c|c|c|}
\hline Sta.\# & Bottle \# & & at. & & ong. & & ate \\
\hline 1 & 24105 & 44 & 59.5 & 66 & 49.2 & 19 & Sept. \\
\hline 2 & & 45 & 01.2 & 66 & 26.5 & 19 & $"$ \\
\hline 3 & & 44 & 58.5 & 66 & 22.3 & 19 & $"$ \\
\hline 4 & & 44 & 55.5 & 66 & 17.8 & 19 & $"$ \\
\hline 5 & & 44 & 52.5 & 66 & 13.0 & 19 & $"$ \\
\hline 6 & & 44 & 49.5 & 66 & 08.0 & 19 & $"$ \\
\hline 7 & 24141 & 44 & 46.5 & 66 & 03.5 & 19 & $"$ \\
\hline 8 & & 44 & 43.5 & 65 & 58.8 & 19 & $"$ \\
\hline 9 & 24149 & 44 & 40.2 & 65 & $54: 0$ & 19 & $"$ \\
\hline 10 & & 44 & 51.0 & 65 & 35.0 & 19 & $"$ \\
\hline 11 & & 44 & 54.2 & 65 & 38.5 & 19 & $"$ \\
\hline 12 & & 44 & 57.5 & 65 & 42.2 & 19 & $"$ \\
\hline 13 & & 45 & 00.8 & 65 & 46.0 & 19 & $"$ \\
\hline 14 & & 45 & 04.0 & 65 & 49.8 & 19 & $"$ \\
\hline 15 & & 45 & 07.0 & 65 & 53.2 & 19 & $"$ \\
\hline 16 & & 45 & 10.0 & 65 & 57.0 & 19 & $"$ \\
\hline 17 & & 45 & 15.5 & 65 & 37.0 & 19 & $"$ \\
\hline 18 & & 45 & 12.5 & 65 & 33.0 & 20 & $"$ \\
\hline 19 & & 45 & 09.0 & 65 & 29.0 & 20 & $"$ \\
\hline 20 & & 45 & 05.8 & 65 & 25.2 & 20 & $"$ \\
\hline 21 & & 45 & 02.5 & 65 & 21.2 & 20 & $"$ \\
\hline 22 & & 44 & 59.5 & 65 & 17.5 & 20 & $"$ \\
\hline 23 & 24234 & 45 & 05.8 & 65 & 03.0 & 20 & $"$ \\
\hline 24 & 24242 & 45 & 09.2 & 65 & 07.0 & 20 & $"$ \\
\hline 25 & 24245 & 45 & 12.5 & 65 & 11.0 & 20 & $"$ \\
\hline 26 & & 45 & 15.8 & 65 & 15.0 & 20 & $"$ \\
\hline 27 & & 45 & 19.0 & 65 & 19.0 & 20 & $"$ \\
\hline 28 & 24266 & 45 & 22.2 & 65 & 22.8 & 20 & $"$ \\
\hline & 24267 & 45 & 22.2 & 65 & 22.8 & 20 & $"$ \\
\hline
\end{tabular}

Recovered

Lat. Long. Date

4501.1

$\begin{array}{lll}66 & 51 & 27 \\ \text { Sept. ' } 56\end{array}$

No Returns



$"$ "

"

$\begin{array}{llllll}44 & 48.5 & 65 & 33 & 20 & \text { Nov. '56 }\end{array}$

No Returns

$45 \quad 10$

6446

6 Nov. ' 56

No Returns

$45 \quad 06$

$45 \quad 11$

$45 \quad 10$

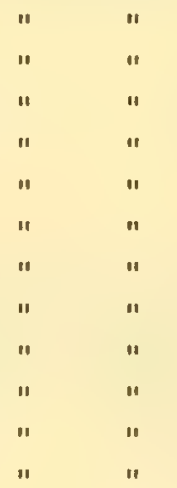

$\begin{array}{llll}64 & 57 \quad 30 \text { Oct. ' } 56\end{array}$ $\begin{array}{lllll}64 & 42 & 17 & \text { Oct. '56 }\end{array}$

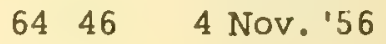
No Returns

$\begin{array}{ll}45 & 20\end{array}$

$45 \quad 20.4$ $\begin{array}{lllll}64 & 27 & 21 \text { Oct. ' } 56\end{array}$ $\begin{array}{lllll}64 & 47.7 & 5 & \text { Oct. ' } 56\end{array}$ 
Released

\begin{tabular}{|c|c|c|c|c|c|c|c|}
\hline \multirow{2}{*}{$\begin{array}{c}\text { Sta.\# } \\
29\end{array}$} & \multirow{2}{*}{$\begin{array}{c}\text { Bottle \# } \\
24272\end{array}$} & \multicolumn{2}{|c|}{ Lat. } & \multicolumn{2}{|c|}{ Long . } & \multicolumn{2}{|c|}{ Late } \\
\hline & & 45 & 21.8 & 65 & 17.0 & 20 & Sept. \\
\hline 30 & & 45 & 21.2 & 65 & 11.0 & 20 & $"$ \\
\hline 31 & 24285 & 45 & 20.8 & 65 & 05.2 & 20 & $"$ \\
\hline 32 & & 42 & 20.2 & 64 & 59.5 & 20 & $"$ \\
\hline 33 & & 45 & 16.2 & 64 & 58.0 & 20 & $"$ \\
\hline 34 & & 45 & 12.5 & 64 & 56.5 & 20 & $"$ \\
\hline 35 & & 45 & 08.5 & 64 & 55.2 & 20 & $"$ \\
\hline 36 & & 44 & 18.0 & 66 & 43.0 & 20 & $"$ \\
\hline \multirow[t]{3}{*}{37} & 24364 & 44 & 07.0 & 66 & 21.0 & 21 & $"$ \\
\hline & 24370 & 44 & 07.0 & 66 & 21.0 & 21 & $"$ \\
\hline & 24372 & 44 & 07.0 & 66 & 21.0 & 21 & $"$ \\
\hline 38 & & 43 & 51.0 & 66 & 45.0 & 21 & $"$ \\
\hline \multirow[t]{2}{*}{39} & 24388 & 43 & 40.0 & 66 & 12.0 & 24 & $"$ \\
\hline & $24390^{\circ}$ & 43 & 40.0 & 66 & 12.0 & 24 & $"$ \\
\hline 40 & 24400 & 43 & 18.0 & 66 & 22.0 & 24 & $"$ \\
\hline \multirow[t]{2}{*}{41} & 24421 & 43 & 18.0 & 65 & 16.0 & 24 & $"$ \\
\hline & 24422 & 43 & 18.0 & 65 & 16.0 & 24 & $"$ \\
\hline 42 & 24430 & 43 & 00.0 & 65 & 16.0 & 25 & $"$ \\
\hline 43 & & 43 & 00.0 & 66 & 31.5 & 25 & $"$ \\
\hline 44 & 24452 & 43 & 41.0 & 67 & 00.0 & 25 & $"$ \\
\hline 45 & & 43 & 41.0 & 68 & 00.0 & 25 & $"$ \\
\hline 46 & & 43 & 22.0 & 68 & 00.0 & 25 & $"$ \\
\hline 47 & 24483 & 43 & 22.0 & 68 & 33.0 & 26 & $"$ \\
\hline 48 & & 43 & 00.0 & 68 & 33.0 & 26 & $"$ \\
\hline 49 & 24512 & 43 & 00.0 & 69 & 31.5 & 26 & $"$ \\
\hline 50 & & 42 & 39.5 & 69 & 31.5 & 26 & $"$ \\
\hline \multirow[t]{7}{*}{51} & 24532 & 41 & 55.5 & 70 & 27.0 & 29 & $"$ \\
\hline & 24534 & 41 & 55.5 & 70 & 27.0 & 29 & $"$ \\
\hline & 24537 & 41 & 55.5 & 70 & 27.0 & 29 & $"$ \\
\hline & 24538 & 41 & 55.5 & 70 & 27.0 & 29 & $"$ \\
\hline & 24539 & 41 & 55.5 & 70 & 27.0 & 29 & $"$ \\
\hline & 24540 & 41 & 55.5 & 70 & 27.0 & 29 & $"$ \\
\hline & 24542 & 41 & 55.5 & 70 & 27.0 & 29 & $"$ \\
\hline \multirow[t]{4}{*}{52} & 24543 & 42 & 20.0 & 70 & 38.5 & 29 & $"$ \\
\hline & 24547 & 42 & 20.0 & 70 & 38.5 & 29 & $"$ \\
\hline & 24550 & 42 & 20.0 & 70 & 38.5 & 29 & $"$ \\
\hline & 24554 & 42 & 20.0 & $7 n$ & 38.5 & 29 & $"$ \\
\hline
\end{tabular}

Recovered

Lat.

Long. Date

$44 \quad 05$

$45 \quad 28$

$\begin{array}{llll}66 & 12.8 & 22 \text { Jan. '57 }\end{array}$ No Returns

$6450 \quad 20$ Oct. '56

No Returns

$\begin{array}{ll}44 & 05\end{array}$

$44 \quad 05$

$44 \quad 05$
-14

$\begin{array}{ll}44 & 17\end{array}$

$44 \quad 52$

$43 \quad 30$

$\begin{array}{ll}43 & 37\end{array}$

$44 \quad 52$

$43 \quad 29$

$43 \quad 29$

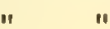

"11

II

" "

$\begin{array}{llll}66 & 11 & 29 & \text { Oct. '56 }\end{array}$

$\begin{array}{llll}66 & 12.5 & 24 & \text { Oct. '56 }\end{array}$

$\begin{array}{llll}66 & 12.5 & 24 & \text { Oct. '56 }\end{array}$ No Returns

$\begin{array}{llll}66 & 08.2 & 12 & \text { Oct. '56 }\end{array}$

$\begin{array}{lll}65 & 27 & 10 \text { Nov. '56 }\end{array}$

$\begin{array}{lll}65 & 32 \quad 12 \text { Dec. '56 }\end{array}$

$\begin{array}{lllll}65 & 46 & 18 & \text { Oct. '56 }\end{array}$

$\begin{array}{lll}65 & 27 \quad 10 \text { Nov. '56 }\end{array}$

$0132 \quad 29$ Jun. '58

No Returns

$6540 \quad 20$ Dec. 156

No Returns

$\begin{array}{llllll}43 & 48.5 & 66 & 09 & 28 \text { Jan. '57 }\end{array}$ No Returns

$\begin{array}{llllll}42 & 01.9 & 70 & 05.6 & 23 & \text { Nov. } ' 56\end{array}$ No Returns

$\begin{array}{lllllll}41 & 59.5 & 70 & 05 & 24 & \text { Oct. } 156\end{array}$

$\begin{array}{llllll}41 & 58 & 70 & 05 & 20 & \text { Oct. '56 }\end{array}$

$\begin{array}{lllllll}42 & 02 & 70 & 05.7 & 13 & \text { Oct. '56 }\end{array}$

$\begin{array}{llllll}41 & 57.5 & 70 & 04.7 & 13 & \text { Oct. '56 }\end{array}$

$\begin{array}{llllll}41 & 47 & 70 & 30 & 19 \text { Oct. '56 }\end{array}$

$4158 \quad 70 \quad 05 \quad 30$ Oct. '56

$\begin{array}{llllll}41 & 55.5 & 70 & 00.8 & 8 & \text { Oct. ' } 56\end{array}$

$\begin{array}{lllll}41 & 17.5 & 70 & 05.7 & 10 \\ \text { Nov. } & 56\end{array}$

$\begin{array}{llllll}41 & 47 & 69 & 56 & 25 & \text { Oct. '56 }\end{array}$

$\begin{array}{llllll}41 & 16 & 70 & 12.5 & 21 & \mathrm{Jan} .\end{array}$

$\begin{array}{lllllll}41 & 35 & 69 & 59 & 27 & \text { Oct. '56 }\end{array}$ 
Released

Recovered

Sta.\# Bottle \# Lat. Long. Date Lat. Long. Date

53

54

55

56

57

58

59

60

61

62

63

64

65

66

67

68

69

70

71

72

73

74

$\mathrm{Y}$

$\mathrm{X}$

Y

C

B

C $\begin{array}{lllll}42 & 42.5 & 70 & 00.0 & 29 \\ \text { Sept. }\end{array}$

24573

24575

24578

24598

$42 \quad 42.5$

$42 \quad 42.5$

$42 \quad 42.5$

4304.5

$43 \quad 22.5$

$43 \quad 22.5$

$43 \quad 41.5$

$43 \quad 41.5$

$44 \quad 00.0$

$44 \quad 00.0$

$44 \quad 23.5$

4400.0

4400.0

4400.0

24682
24683

24684

24689

$44 \quad 31.5$

$44 \quad 53.8$

$\begin{array}{ll}44 & 57.8\end{array}$

$\begin{array}{ll}44 & 57.8\end{array}$

$44 \quad 49.2$

$44 \quad 44.8$

$\begin{array}{ll}44 & 40.5\end{array}$

$44 \quad 35.8$

$44 \quad 31.5$

$44 \quad 27.0$

$\begin{array}{ll}44 & 22.5\end{array}$

15921

$44 \quad 18.0$

$43 \quad 55$

$43 \quad 49$

$43 \quad 55$

$44 \quad 40$

$44 \quad 45.8$

$44 \quad 40$

\section{$\begin{array}{ll}70 & 31\end{array}$}

$\begin{array}{ll}70 & 31\end{array}$

$\begin{array}{ll}70 & 31\end{array}$

$\begin{array}{ll}70 & 00.0\end{array}$

$\begin{array}{ll}70 & 20.0\end{array}$

$\begin{array}{ll}69 & 00.0\end{array}$

6900.0

$68 \quad 34.0$

$\begin{array}{ll}68 & 34.0\end{array}$

$\begin{array}{ll}67 & 37.0\end{array}$

$\begin{array}{ll}67 & 37.0\end{array}$

$\begin{array}{ll}67 & 00.0\end{array}$

$\begin{array}{ll}67 & 00.0\end{array}$

$\begin{array}{ll}67 & 00.0\end{array}$

$\begin{array}{ll}67 & 11.5\end{array}$

$\begin{array}{ll}66 & 45.0\end{array}$

$\begin{array}{ll}66 & 51.0\end{array}$

$\begin{array}{ll}66 & 51.0\end{array}$

$\begin{array}{ll}66 & 42.0\end{array}$

$\begin{array}{ll}66 & 38.8\end{array}$

$\begin{array}{ll}66 & 35.8\end{array}$

$\begin{array}{ll}66 & 32.8\end{array}$

$\begin{array}{ll}66 & 29.5\end{array}$

$\begin{array}{ll}66 & 26.5\end{array}$

$\begin{array}{ll}66 & 23.5\end{array}$

$\begin{array}{ll}66 & 20.5\end{array}$

$\begin{array}{ll}66 & 23.2\end{array}$

$\begin{array}{ll}66 & 31\end{array}$

$\begin{array}{ll}66 & 23\end{array}$

$\begin{array}{ll}67 & 00\end{array}$

$66 \quad 55$

6700

29 "

29 "

29 "

30 "

$30 \quad$

$30 "$

$30 "$

30

30

30 "

1 Oct.

1 "

1 "

1 "

1 "

$$
1 \text { " }
$$

1 "

1

4 "

4 "

4 "

4 "

4 "

$$
4 \text { " }
$$

4 "

4 "

7

7
7
9

$1 "$

$41 \quad 48$

4151

4154

$42 \quad 28$

\section{No Returns}

$\begin{array}{rrrr}69 & 56 & 27 \text { Oct. '56 } \\ 69 & 57 & 28 \text { Oct. '56 } \\ 69 & 58 & 4 \text { Nov. '56 }\end{array}$

No Returns $\begin{array}{lllll}70 & 55 & 31 \text { Oct. '56 }\end{array}$ No Returns
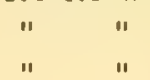

"

"

"

$\begin{array}{lllll}44 & 50.8 & 65 & 29 & 16 \text { Nov. '56 }\end{array}$

$\begin{array}{llllll}44 & 40 & 66 & 43.5 & 28 & \text { Mar. } 57\end{array}$

$\begin{array}{lllllll}44 & 24 & 66 & 14 & 13 & \text { Oct. '56 }\end{array}$

$\begin{array}{lllll}43 & 59.5 & 66 & 09 & 14 \mathrm{Dec} .\end{array}$

No Returns

$\begin{array}{llllll}44 & 39.5 & 66 & 47.5 & 10 & \text { Oct. } 156\end{array}$

$\begin{array}{lllll}44 & 56.8 & 65 & 15.8 & 5\end{array}$ Dec.' 56

$\begin{array}{lllll}44 & 53 & 65 & 24 & 9\end{array}$ Nov.' 56

4420

No Rețurns

$\begin{array}{lll}66 & 07.5 & 3 \text { Dec. } 57\end{array}$

No Returns
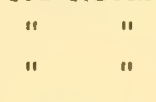

$44 \quad 37$

$6555 \quad 15$ Oct. ' 56
No Returns


Silver Bay Cruise 1

21 September - 5 October

1956

12 bottles dropped at each station, all ballasted.

Released

\begin{tabular}{|c|c|c|c|c|c|c|c|}
\hline Sta.\# & Bottle \# & & at. & & ng. & & ate \\
\hline 1 & 25806 & 43 & 40 & 69 & 46.2 & 21 & Sept. \\
\hline 7 & & 43 & 25.8 & 68 & 31 & 21 & $"$ \\
\hline 16 & & 43 & 07.5 & 66 & 58.2 & 22 & $"$ \\
\hline 22 & & 42 & 41.7 & 67 & 00 & 22 & $"$ \\
\hline 28 & & 42 & 31.8 & 68 & 03 & 22 & $"$ \\
\hline 38 & 25868 & 42 & 29.5 & 69 & 29 & 23 & $"$ \\
\hline & 25871 & 42 & 29.5 & 69 & 29 & 23 & $"$ \\
\hline $\mathrm{X}$ & 25877 & 42 & 07 & 69 & 30.8 & 23 & $"$ \\
\hline 46 & & 42 & 12.7 & 68 & 33.2 & 24 & $"$ \\
\hline 51 & 25902 & 41 & 59.5 & 68 & 01 & 24 & $"$ \\
\hline 54 & 25914 & 42 & 14.5 & 67 & 28.7 & 24 & $"$ \\
\hline 57 & & 42 & 07.5 & 67 & 04.2 & 24 & $"$ \\
\hline 61 & & 42 & 22.7 & 66 & 31 & 24 & $"$ \\
\hline 65 & & 42 & 02.5 & 66 & 00 & 24 & $"$ \\
\hline 68 & & 42 & 10 & 65 & 36.2 & 25 & $"$ \\
\hline 71 & & 41 & 46.5 & 65 & 35.5 & 25 & $"$ \\
\hline 79 & & 41 & 46.5 & 67 & 02.7 & 25 & $"$ \\
\hline 85 & & 41 & 52 & 67 & 56.5 & 26 & $"$ \\
\hline 91 & & 41 & 48.5 & 69 & 01 & 26 & $"$ \\
\hline 95 & 26018 & 41 & 37 & 69 & 44.7 & 26 & $"$ \\
\hline & 26023 & 41 & 37 & 69 & 44.7 & 26 & $"$ \\
\hline & 26025 & 41 & 37 & 69 & 44.7 & 26 & $"$ \\
\hline & 26026 & 41 & 37 & 69 & 44.7 & 26 & $"$ \\
\hline & 26027 & 41 & 37 & 69 & 44.7 & 26 & $"$ \\
\hline & 26029 & 41 & 37 & 69 & 44.7 & 26 & $"$ \\
\hline 103 & & 41 & 25.5 & 68 & 37 & 26 & $"$ \\
\hline 109 & & 40 & 50.5 & 69 & 06.5 & 29 & $"$ \\
\hline 115 & & 41 & 15 & 69 & 00 & 30 & $"$ \\
\hline 121 & & 41 & 15.7 & 68 & 00 & 30 & $"$ \\
\hline
\end{tabular}

Recovered

Lat.

Long.

Date

$\begin{array}{llllll}42 & 04.5 & 70 & 13.2 & 11 & \text { Nov. '56 }\end{array}$

No Returns

\begin{tabular}{llllll}
41 & 59.5 & 70 & 04.8 & $18 \mathrm{Jan}$. & \\
\hline & 57
\end{tabular}

\begin{tabular}{llllll}
41 & 23 & 70 & 30.3 & 30 \\
\hline
\end{tabular}

$\begin{array}{lllll}42 & 04.9 & 70 & 11.9 & 29 \text { Sept. '56 }\end{array}$

No Returns

$\begin{array}{lllll}32 & 17.8 & 64 & 48.2 & 8 \\ 3 & \text { May '58 }\end{array}$

$\begin{array}{lllll}32 & 14.9 & 64 & 49.7 & 6 \text { Apr. '57 }\end{array}$

No Returns

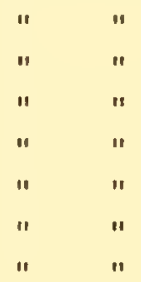

$\begin{array}{llllll}41 & 18 & 70 & 05 & 4 & \text { Oct. '56 }\end{array}$

\begin{tabular}{llllll}
41 & 20 & 70 & 02 & 28 & $\mathrm{Jul}$. \\
\hline
\end{tabular}

$\begin{array}{llllll}41 & 18.2 & 70 & 01.7 & 19 & \text { Mar. '57 }\end{array}$

\begin{tabular}{llllll}
41 & 23.5 & 70 & 02.8 & 23 & $\mathrm{Dec}$. \\
\hline
\end{tabular}

$\begin{array}{lllll}41 & 21.6 & 70 & 08.1 & 5 \\ \text { May '58 }\end{array}$

$\begin{array}{lllllll}41 & 19 & 70 & 03 & 27 & \text { Oct. '56 }\end{array}$

No Returns 
Released

\begin{tabular}{|c|c|c|c|c|c|c|c|}
\hline Sta.\# & Bottle \# & \multicolumn{2}{|c|}{ Lat. } & \multicolumn{2}{|c|}{ Long. } & \multicolumn{2}{|c|}{ Date } \\
\hline 127 & & 41 & 14 & 67 & 02 & 30 & Sept. \\
\hline 133 & & 41 & 15 & 66 & 12.5 & 30 & $"$ \\
\hline 139 & & 40 & 40 & 66 & 59 & 1 & Oct. \\
\hline 144 & 26116 & 40 & 39.5 & 68 & 00.5 & 1 & $"$ \\
\hline 149 & & 40 & 38.5 & 68 & 59 & 1 & $"$ \\
\hline 155 & & 40 & 40.5 & 69 & 58.7 & 1 & $"$ \\
\hline 159 & 26161 & 40 & 00 & 70 & 00 & 1 & $"$ \\
\hline 165 & & 39 & 58.5 & 68 & 51 & 2 & $"$ \\
\hline 171 & & 40 & 03 & 67 & 54 & 2 & $"$ \\
\hline 173 & & 40 & 25 & 67 & 48 & 2 & $"$ \\
\hline 178 & & 40 & 57 & 67 & 27 & 2 & $"$ \\
\hline 182 & & 41 & 29.5 & 67 & 04.5 & 2 & $"$ \\
\hline 186 & & 41 & 59.5 & 66 & 49 & 3 & $"$ \\
\hline 192 & & 42 & 40.7 & 66 & 30.5 & 3 & $"$ \\
\hline 202 & & 42 & 31.5 & 65 & 03.5 & 3 & $"$ \\
\hline 210 & & 42 & 40.5 & 66 & 59 & 4 & $"$ \\
\hline 215 & & 42 & 47.2 & 68 & 00.5 & 4 & $"$ \\
\hline 220 & & 42 & 53.5 & 68 & 59 & 5 & $"$ \\
\hline 228 & 26294 & 43 & 00 & 70 & 27.5 & 5 & $"$ \\
\hline & 26295 & 43 & 00 & 70 & 27.5 & 5 & $"$ \\
\hline & 26296 & 43 & 00 & 70 & 27.5 & 5 & $"$ \\
\hline & 26297 & 43 & 00 & 70 & 27.5 & 5 & $"$ \\
\hline & 26304 & 43 & 00 & 70 & 27.5 & 5 & $"$ \\
\hline
\end{tabular}

Recovered

Lat. Long . Date

\section{No Returns}

" "

"

$5544 \quad 08 \quad 08 \mathrm{E} \quad 1$ Jan. '59 No Returns

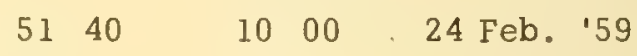
No Returns

$\begin{array}{ll}\text { " } & \text { " } \\ \text { " } & \text { " } \\ \text { " } & \text { " } \\ \text { " } & \text { " } \\ \text { " } & \text { " } \\ \text { " } & \text { " } \\ \text { " } & \text { " }\end{array}$

$\begin{array}{lllll}42 & 18 & 70 & 53 & 4 \text { Nov. '56 }\end{array}$

$\begin{array}{lllll}42 & 18.5 & 70 & 53 & 3 \text { Nov. '56 }\end{array}$

$\begin{array}{llllll}41 & 59.5 & 70 & 04.3 & 28 & \text { Aug. '57 }\end{array}$

$\begin{array}{llllll}42 & 03 & 70 & 13 & 26 \mathrm{Jul} . & 157\end{array}$

$\begin{array}{llllll}42 & 22 & 70 & 58 & 29 & \text { Oct. '56 }\end{array}$ 
Silver Bay Cruise 2

$15-25$ October

1956

12 bottles dropped at each station, all ballasted.

Released

\begin{tabular}{|c|c|c|c|c|c|c|c|}
\hline Sta.\# & Bottle \# & & at. & & ng. & & ate \\
\hline 1 & & 43 & 39.9 & 69 & 45 & 15 & Oct. \\
\hline 3 & 26325 & 43 & 25.5 & 69 & 59.2 & 15 & $"$ \\
\hline 7 & 26339 & 43 & 00 & 70 & 27.5 & 16 & $"$ \\
\hline & 26341 & 43 & 00 & 70 & 27.5 & 16 & $"$ \\
\hline 10 & 26342 & 42 & 57.2 & 70 & 00 & 16 & $"$ \\
\hline & 26344 & 42 & 57.2 & 70 & 00 & 16 & $"$ \\
\hline & 26350 & 42 & 57.2 & 70 & 00 & 16 & $"$ \\
\hline & 26351 & 42 & 57.2 & 70 & 00 & 16 & $"$ \\
\hline & 26353 & 42 & 57.2 & 70 & 00 & 16 & " \\
\hline 15 & & 42 & 52 & 69 & 01.8 & 16 & $"$ \\
\hline 19 & & 42 & 46.2 & 68 & 00 & 16 & $"$ \\
\hline 24 & & 42 & 40 & 66 & 59.8 & 16 & $"$ \\
\hline 29 & 26392 & 42 & 35.4 & 66 & 04.4 & 17 & $"$ \\
\hline & 26396 & 42 & 35.4 & 66 & 04.4 & 17 & $"$ \\
\hline 34 & & 42 & 29.8 & 65 & 00 & 17 & $"$ \\
\hline 40 & 26416 & 43 & 12 & 65 & 49.2 & 17 & $"$ \\
\hline & 26421 & 43 & 12 & 65 & 49.2 & 17 & $"$ \\
\hline 43 & 26430 & 43 & 10 & 66 & 08.2 & 17 & $"$ \\
\hline 47 & 26443 & 42 & 37.8 & 66 & 28.2 & 17 & $"$ \\
\hline 53 & & 41 & 48 & 66 & 57.9 & 18 & " \\
\hline 57 & & 41 & 16.2 & 67 & 15.9 & 18 & $"$ \\
\hline 59 & & 40 & 58.5 & 67 & 26.5 & 18 & $"$ \\
\hline 63 & & 40 & 25.2 & 67 & 44 & 18 & " \\
\hline 66 & & 40 & 01 & 67 & 57 & 18 & 11 \\
\hline 71 & & 40 & 00.5 & 68 & 48.5 & 18 & " \\
\hline 75 & & 40 & 02 & 69 & 58 & 19 & " \\
\hline 79 & & 40 & 37 & 70 & 05.5 & 19 & $"$ \\
\hline 85 & & 40 & 38.5 & 69 & 02 & 20 & $"$ \\
\hline 92 & & 40 & 40.2 & 67 & 53.4 & 20 & " \\
\hline 97 & & 40 & 40.5 & 66 & 59 & 20 & " \\
\hline 98 & & 40 & 40 & 66 & 52 & 20 & " \\
\hline
\end{tabular}

Recovered

Lat. Long. Date

No Returns

$\begin{array}{lllll}43 & 10.6 & 70 & 36.5 & 2 \text { Nov. '56 }\end{array}$

$\begin{array}{lllll}42 & 39.5 & 70 & 35.9 & 4 \text { Nov. '56 }\end{array}$

$\begin{array}{lllll}42 & 36 & 70 & 40 & 14 \\ & \text { Nov. '56 }\end{array}$

$\begin{array}{lllll}42 & 14.5 & 70 & 46 & 29 \text { Nov. ' } 57\end{array}$

$\begin{array}{lllll}42 & 17.7 & 70 & 53.2 & 12 \text { Nov. '56 }\end{array}$

$\begin{array}{lllll}42 & 16.5 & 70 & 53 & \end{array}$

$\begin{array}{lllll}42 & 25.9 & 70 & 56.2 & 22 \\ 40 v . ~ ' 56\end{array}$

$\begin{array}{lllll}42 & 18 & 70 & 55 & 5\end{array}$ Nov. '56

No Returns

\begin{tabular}{lllll}
4500 & 64 & 56.5 & 24 & $\mathrm{Dec}$. \\
\hline
\end{tabular}

$4456.8 \quad 65 \quad 15.8 \quad 5$ Dec. '56 No Returns

$\begin{array}{llllll}44 & 24 & 66 & 13 & 9 & \text { Jan. '57 }\end{array}$

$\begin{array}{lllll}44 & 29 & 65 & 57 & 24 \text { Nov. '56 }\end{array}$

$\begin{array}{llllll}43 & 49 & 66 & 08 & 29 & \text { Mar. '57 }\end{array}$

$\begin{array}{lllll}44 & 17.2 & 66 & 19.6 & 3 \\ \text { Jan. } & 57\end{array}$




Released

\begin{tabular}{|c|c|c|c|c|c|c|c|}
\hline Sta.\# & Bottle \# & & at. & & ng. & & ate \\
\hline 104 & & 41 & 16 & 66 & 13.5 & 20 & Oct. \\
\hline 108 & & 41 & 15.5 & 66 & 59.5 & 21 & $"$ \\
\hline 112 & & 41 & 15.5 & 68 & 00 & 21 & $"$ \\
\hline 118 & & 41 & 17.3 & 69 & 01.5 & 21 & $"$ \\
\hline 120 & & 41 & 13.8 & 69 & 22.3 & 21 & $"$ \\
\hline 123 & & 40 & 52 & 69 & 05.2 & 21 & $"$ \\
\hline 127 & & 41 & 26 & 68 & 38 & 21 & $"$ \\
\hline 133 & 26679 & 41 & 37.2 & 69 & 46 & 22 & $"$ \\
\hline & 26680 & 41 & 37.2 & 69 & 46 & 22 & $"$ \\
\hline & 26688 & 41 & 37.2 & 69 & 46 & 22 & $"$ \\
\hline 136 & & 41 & 46.5 & 69 & 04.5 & 22 & $"$ \\
\hline 141 & & 41 & 50.5 & 68 & 01 & 22 & $"$ \\
\hline 154 & & 41 & 47 & 65 & 36.5 & 23 & $"$ \\
\hline 156 & 26730 & 42 & 07.9 & 65 & 38.5 & 23 & $"$ \\
\hline 158 & & 42 & 03.5 & 66 & 00.5 & 23 & $"$ \\
\hline 161 & & 42 & 21 & 66 & 31.5 & 23 & $"$ \\
\hline 165 & & 42 & 02.3 & 67 & 00 & 23 & $"$ \\
\hline 167 & & 42 & 17.5 & 67 & 22 & 23 & $"$ \\
\hline 172 & 26796 & 42 & 05.5 & 67 & 58 & 23 & $"$ \\
\hline 176 & & 42 & 09.5 & 68 & 28.7 & 24 & $"$ \\
\hline 180 & 26816 & 42 & 10.8 & 69 & 30.6 & 24 & $"$ \\
\hline 182 & & 42 & 29.2 & 69 & 26 & 24 & $"$ \\
\hline 190 & & 42 & 31 & 68 & 00.8 & 24 & $"$ \\
\hline 195 & & 42 & 29.5 & 67 & 02.2 & 24 & $"$ \\
\hline 201 & 26868 & 43 & 08.8 & 67 & 15 & 25 & " \\
\hline 206 & & 43 & 22 & 68 & 01.2 & 25 & $"$ \\
\hline 209 & 26891 & 43 & 27.6 & 68 & 36.5 & 25 & $"$ \\
\hline 211 & & 43 & 32 & 69 & 01 & 25 & $"$ \\
\hline
\end{tabular}

Recovered

Lat. Long. Date

No Returns

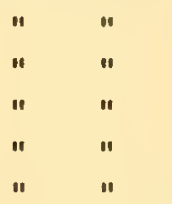

$\begin{array}{llllll}41 & 25 & 70 & 33 & 2 & \text { May '57 } \\ 41 & 21 & 70 & 27 & 1 & \text { Nov. '56 } \\ 41 & 24 & 70 & 27 & 1 \text { Nov. '56 }\end{array}$

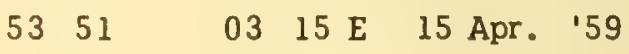
No Returns

" "

"

"

$\begin{array}{llllll}44 & 17.5 & 66 & 19 & 5 \text { Feb. } 57\end{array}$ No Returns

$\begin{array}{llllll}41 & 48 & 69 & 56 & 5 & \text { Jan. '57 }\end{array}$ No Returns

" "

$\begin{array}{llllll}41 & 49.3 & 70 & 00.3 & 23 & \text { Mar. '58 }\end{array}$ No Returns

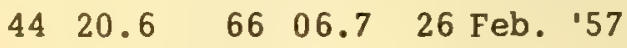
No Returns 
Harengus Cruise AH6

$16-25$ October $\quad 2-8$ November

1956

Stations 1-35, 67-74, X, Y, and Z; 6 bottles dropped, all ballasted.

Stations 36-66; 12 bottles dropped, even numbered bottles ballasted, odd numbered bottles not ballasted.

Released

Sta.\# Bottle \# Lat. Long. Date

$\begin{array}{llllll}44 & 59.5 & 66 & 49.2 & 16 & \text { Oct. } \\ 45 & 01.2 & 66 & 26.5 & 16 & " \\ 44 & 58.5 & 66 & 22.5 & 16 & " \\ 44 & 55.5 & 66 & 17.8 & 16 & " \\ 44 & 52.5 & 66 & 13 & 16 & " \\ 44 & 49.5 & 66 & 08 & 16 & " \\ 44 & 46.5 & 66 & 03.5 & 16 & " \\ 44 & 43.5 & 65 & 58.8 & 16 & " \\ 44 & 40.2 & 65 & 54 & 16 & " \\ 44 & 51.0 & 65 & 35.0 & 16 & " \\ 44 & 54.2 & 65 & 38.5 & 16 & " \\ 44 & 57.5 & 65 & 42.2 & 16 & " \\ 45 & 00.8 & 65 & 46.0 & 16 & " \\ 45 & 04.0 & 65 & 49.8 & 16 & " \\ 45 & 07.0 & 65 & 53.2 & 16 & " \\ 45 & 10.0 & 65 & 57.0 & 16 & " \\ 45 & 15.5 & 65 & 37.0 & 16 & " \\ 45 & 12.5 & 65 & 33.0 & 17 & " \\ 45 & 09.0 & 65 & 29.0 & 17 & " \\ 45 & 05.8 & 65 & 25.2 & 17 & " \\ 45 & 02.5 & 65 & 21.2 & 17 & " \\ 44 & 59.2 & 65 & 17.5 & 17 & " \\ 45 & 05.8 & 65 & 03.0 & 17 & " \\ 45 & 09.2 & 65 & 07.0 & 17 & " \\ 45 & 12.5 & 65 & 11.0 & 17 & " \\ 45 & 15.8 & 65 & 15.0 & 17 & " \\ 45 & 15.8 & 65 & 15.0 & 17 & " \\ 45 & 15.8 & 65 & 15.0 & 17 & "\end{array}$

\section{6}

6515.

$\begin{array}{lll}24864 & 45 & 09.0 \\ & 45 & 05.8 \\ & 45 & 02.5 \\ & 44 & 59.2 \\ & 45 & 05.8 \\ & 45 & 09.2 \\ & 45 & 12.5 \\ 24901 & 45 & 15.8 \\ 24903 & 45 & 15.8 \\ 24904 & 45 & 15.8\end{array}$

24904
Recovered

Lat. Long. Date

No Returns

4454

$$
\begin{array}{ll}
\text { " } & \text { " } \\
\text { " } & \text { " } \\
\text { " } & \text { " } \\
\text { " } & \text { " } \\
\text { " } & \text { " }
\end{array}
$$


Harengus Cruise AH6 (cont'd)

Released

\begin{abstract}
Sta.\# Bottle \#
\end{abstract}

\begin{tabular}{|c|c|c|c|c|c|c|c|}
\hline 27 & & 45 & 19.0 & 65 & 19.0 & 17 & Oct. \\
\hline 28 & & 45 & 22.2 & 65 & 22.8 & 17 & $"$ \\
\hline 29 & 24922 & 45 & 21.8 & 65 & 17 & 17 & " \\
\hline 30 & & 45 & 21.2 & 65 & 11.0 & 17 & - \\
\hline 31 & & 45 & 20.8 & 65 & 05.2 & 17 & $"$ \\
\hline 32 & & 45 & 20.2 & 64 & 59.5 & 17 & $"$ \\
\hline 33 & & 45 & 16.2 & 64 & 58.0 & 17 & $"$ \\
\hline \multirow[t]{2}{*}{34} & 24950 & 45 & 12.5 & 64 & 56.5 & 17 & $"$ \\
\hline & 24954 & 45 & 12.5 & 64 & 56.5 & 17 & $"$ \\
\hline 35 & \multirow[t]{6}{*}{24959} & 45 & 08.5 & 64 & 55.2 & 17 & $"$ \\
\hline 74 & & 44 & 18.1 & 66 & 21.0 & 17 & " \\
\hline 73 & & 44 & 22.5 & 66 & 23.5 & 17 & $"$ \\
\hline 72 & & 44 & 27.0 & 66 & 26.5 & 17 & $"$ \\
\hline 71 & & 44 & 31.5 & 66 & 29.5 & 17 & $"$ \\
\hline 70 & & 44 & 35.8 & 66 & 32.8 & 17 & $"$ \\
\hline 69 & \multirow[t]{4}{*}{24933} & 44 & 40.5 & 66 & 35.8 & 17 & $"$ \\
\hline 68 & & 44 & 44.8 & 66 & 38.8 & 17 & $"$ \\
\hline 67 & & 44 & 49.2 & 66 & 42.0 & 17 & $"$ \\
\hline 36 & & 44 & 18.0 & 66 & 43.0 & 18 & $"$ \\
\hline 37 & \multirow[t]{13}{*}{25027} & 44 & 07.0 & 66 & 21.0 & 18 & $"$ \\
\hline 38 & & 43 & 51.0 & 66 & 45.0 & 18 & $"$ \\
\hline 39 & & 43 & 40 & 66 & 12.0 & 18 & $"$ \\
\hline 40 & & 43 & 18.0 & 66 & 22.0 & 18 & " \\
\hline 41 & & 43 & 18.0 & 65 & 16.0 & 18 & $"$ \\
\hline 42 & & 43 & 00.0 & 65 & 16.0 & 22 & $"$ \\
\hline 43 & & 43 & 00.0 & 66 & 31.5 & 22 & $"$ \\
\hline 44 & & 43 & 41.0 & 67 & 00.0 & 23 & $"$ \\
\hline 45 & & 43 & 41.0 & 68 & 00.0 & 23 & " \\
\hline 46 & & 43 & 22.0 & 68 & 00.0 & 23 & $"$ \\
\hline 47 & & 43 & 22.0 & 68 & 33.0 & 23 & $"$ \\
\hline 48 & & 43 & 00.0 & 68 & 33.0 & 23 & $"$ \\
\hline 49 & & 43 & 00.0 & 69 & 31.5 & 24 & $"$ \\
\hline \multirow[t]{2}{*}{50} & 25184 & 42 & 39.5 & 69 & 31.5 & 24 & $"$ \\
\hline & 25188 & 42 & 39.5 & 69 & 31.5 & 24 & $"$ \\
\hline \multirow[t]{3}{*}{51} & 25189 & 41 & 55.5 & 70 & 27.0 & 24 & $"$ \\
\hline & 25190 & 41 & 55.5 & 70 & 27.0 & 24 & $"$ \\
\hline & 25191 & 41 & 55.5 & 70 & 27.0 & 24 & $"$ \\
\hline
\end{tabular}

Recovered

Lat. Long. Date

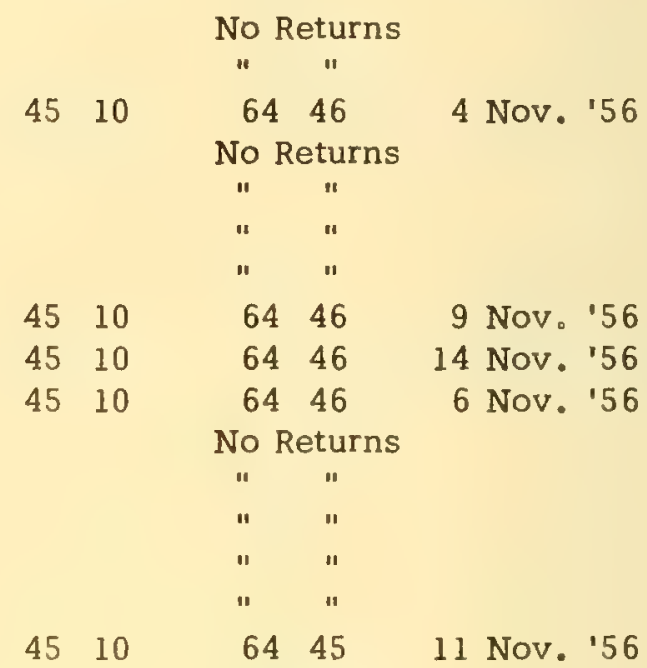

No Returns

$\begin{array}{lllll}44 & 11.5 & 66 & 10 & 12 \mathrm{Nov} \cdot \text { '56 }\end{array}$ No Returns

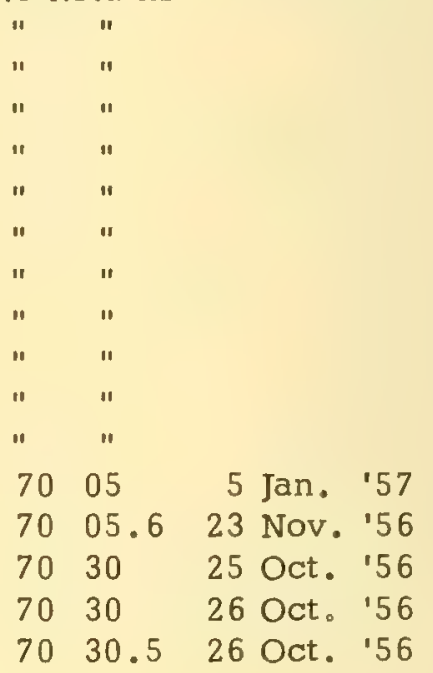


Harengus Cruise AH6 (cont'd)

Released

\begin{tabular}{|c|c|c|c|c|c|c|c|}
\hline \multirow{2}{*}{$\begin{array}{c}\text { Sta.\# } \\
51\end{array}$} & \multirow{2}{*}{$\begin{array}{c}\text { Bottle \# } \\
25195\end{array}$} & \multicolumn{2}{|c|}{ Lat. } & \multicolumn{2}{|c|}{ Long. } & \multicolumn{2}{|c|}{ Date } \\
\hline & & 41 & 55.5 & 70 & 27.0 & 24 & Oct. \\
\hline & 25197 & 41 & 55.5 & 70 & 27.0 & 24 & $"$ \\
\hline & 25200 & 41 & 55.5 & 70 & 27.0 & 24 & $"$ \\
\hline 52 & 25201 & 42 & 20.0 & 70 & 38.5 & 24 & $"$ \\
\hline & 25210 & 42 & 20.0 & 70 & 38.5 & 24 & $"$ \\
\hline 53 & & 42 & 42.5 & 70 & 00.0 & 26 & $"$ \\
\hline 54 & & 42 & 43.0 & 70 & 19.1 & 26 & $"$ \\
\hline 55 & & 43 & 04.5 & 70 & 00.0 & 26 & $"$ \\
\hline 56 & & 43 & 22.5 & 70 & 20.0 & 26 & $"$ \\
\hline 57 & & 43 & 22.5 & 69 & 00.0 & 27 & $"$ \\
\hline 58 & & 43 & 41.5 & 69 & 00.0 & 27 & $"$ \\
\hline 59 & & 43 & 41.5 & 68 & 34.0 & 27 & $"$ \\
\hline 60 & & 44 & 00.0 & 68 & 34.0 & 27 & $"$ \\
\hline 61 & & 44 & 00.0 & 67 & 37.0 & 27 & $"$ \\
\hline 62 & & 44 & 23.5 & 67 & 37.0 & 27 & $"$ \\
\hline 63 & & 44 & 00.0 & 67 & 00.0 & 28 & $"$ \\
\hline 64 & & 44 & 31.5 & 67 & 11.5 & 28 & $"$ \\
\hline 65 & & 44 & 53.8 & 66 & 45.0 & 28 & $"$ \\
\hline 66 & 25379 & 44 & 57.8 & 66 & 51.0 & 28 & $"$ \\
\hline $\mathrm{Z}$ & & 44 & 01 & 66 & 16 & 2 & Nov. \\
\hline$Y$ & & 43 & 55 & 66 & 23 & 2 & $"$ \\
\hline $\mathrm{X}$ & & 43 & 49 & 66 & 31 & 2 & $"$ \\
\hline $\mathrm{Z}$ & & 44 & 01 & 66 & 16 & 2 & $"$ \\
\hline$Y$ & & 43 & 55 & 66 & 23 & 2 & $"$ \\
\hline $\mathrm{Y}$ & 15683 & 43 & 55 & 66 & 23 & 5 & $"$ \\
\hline & 15684 & 43 & 55 & 66 & 23 & 5 & $"$ \\
\hline $\mathrm{Z}$ & & 44 & 01 & 66 & 16 & 8 & $"$ \\
\hline
\end{tabular}

Recovered

Lat. Long. Date

$\begin{array}{lllllll}41 & 46.9 & 70 & 30.1 & 26 & \text { Oct. '56 }\end{array}$

$\begin{array}{lllllll}41 & 47 & 70 & 30 & 25 & \text { Oct. '56 }\end{array}$

$\begin{array}{llllll}41 & 46.7 & 70 & 29.9 & 26 & \text { Oct. } 56\end{array}$

$\begin{array}{llllll}42 & 08.5 & 70 & 41.5 & 25 & \text { Oct. '56 }\end{array}$

$\begin{array}{lllllll}42 & 08.5 & 70 & 41.5 & 27 & \text { Oct. '56 }\end{array}$

No Returns

$$
\begin{array}{ll}
\text { " } & \text { " } \\
\text { " } & \text { " } \\
\text { " } & \text { " } \\
\text { " } & \text { " } \\
\text { " } & \text { " } \\
\text { " } & \text { " } \\
\text { " } & \text { " } \\
\text { " } & \text { " }
\end{array}
$$

$\begin{array}{llllll}44 & 51 & 67 & 00 & 24 & \text { Feb. } 57\end{array}$ No Returns

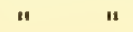

"1

11 11

"

$\begin{array}{llllll}44 & 25 & 66 & 03 & 14 \text { Nov. '56 }\end{array}$

$\begin{array}{lllll}44 & 11.2 & 66 & 10.3 & 28 \\ \text { Nov. '56 }\end{array}$ No Returns 
Sllver Bay Cruise 4

14 - 30 November

1956

12 bottles dropped at each station, all ballasted.

Released

\begin{tabular}{|c|c|c|c|c|c|c|c|}
\hline \multirow{2}{*}{$\begin{array}{c}\text { Sta.\# } \\
1\end{array}$} & \multirow{2}{*}{$\begin{array}{c}\text { Bottle \# } \\
27008\end{array}$} & \multicolumn{2}{|c|}{ Lat. } & \multicolumn{2}{|c|}{ Long. } & \multicolumn{2}{|c|}{ Date } \\
\hline & & 43 & 39.5 & 69 & 47 & 14 & Nov. \\
\hline & 27010 & 43 & 39.5 & 69 & 47 & 14 & $"$ \\
\hline 3 & & 43 & 25.2 & 69 & 58.2 & 14 & $"$ \\
\hline 7 & & 42 & 54.2 & 70 & 14.5 & 15 & $"$ \\
\hline 9 & & 42 & 54.6 & 69 & 59 & 15 & $"$ \\
\hline 13 & & 42 & 52.6 & 69 & 00.5 & 15 & $"$ \\
\hline 18 & & 42 & 48.8 & 68 & 03.3 & 15 & $"$ \\
\hline 24 & & 42 & 36.5 & 66 & 48 & 15 & $"$ \\
\hline 26 & & 42 & 37.8 & 66 & 28.8 & 15 & $"$ \\
\hline 28 & & 42 & 33.5 & 66 & 03 & 16 & $"$ \\
\hline 34 & & 42 & 33 & 65 & 02 & 16 & $"$ \\
\hline 40 & 27131 & 43 & 15 & 65 & 49.8 & 16 & $"$ \\
\hline 42 & 27137 & 43 & 09.8 & 66 & 08 & 16 & $"$ \\
\hline 59 & 27145 & 41 & 16.5 & 67 & 16 & 17 & $"$ \\
\hline 69 & & 40 & 01 & 67 & 52.2 & 17 & $"$ \\
\hline 75 & & 40 & 00.5 & 68 & 50 & 17 & $"$ \\
\hline 81 & & 40 & 06.5 & 69 & 51.8 & 17 & $"$ \\
\hline 87 & 27198 & 40 & 44 & 70 & 05.8 & 18 & $"$ \\
\hline & 27199 & 40 & 44 & 70 & 05.8 & 18 & $"$ \\
\hline 95 & & 40 & 37.7 & 69 & 02.6 & 18 & $"$ \\
\hline 102 & & 40 & 39 & 68 & 00 & 19 & $"$ \\
\hline 108 & & 40 & 43 & 67 & 01.5 & 19 & $"$ \\
\hline 109 & & 40 & 41.3 & 66 & 50.2 & 19 & $"$ \\
\hline 115 & & 41 & 16.5 & 66 & 14.5 & 19 & $"$ \\
\hline 119 & & 41 & 20.2 & 67 & 08 & 19 & $"$ \\
\hline 123 & & 43 & 26 & 68 & 59.5 & 23 & $"$ \\
\hline 125 & & 43 & 24 & 68 & 31.2 & 23 & $"$ \\
\hline 133 & 27308 & 43 & 10.2 & 66 & 58 & 24 & $"$ \\
\hline 140 & & 42 & 26.5 & 66 & 58 & 24 & $"$ \\
\hline 146 & & 42 & 29.5 & 67 & 57 & 25 & $"$ \\
\hline
\end{tabular}

Recovered

Lat. Long. Date

$\begin{array}{llllll}43 & 42 & 69 & 53.7 & 27 & \text { Jul. }\end{array}$

$\begin{array}{llllll}43 & 45.1 & 69 & 46.6 & 18 \text { Jun. '59 }\end{array}$ No Returns

$\begin{array}{ll}\text { " } & \text { " } \\ \text { " } & \text { " } \\ \text { " } & \text { " } \\ \text { " } & \text { " } \\ \text { " } & \text { " }\end{array}$

$\begin{array}{lllllll}45 & 17.5 & 64 & 47 & 7 & \text { Jul. } & 157\end{array}$

$\begin{array}{llllll}44 & 48.8 & 65 & 32.3 & 11 & \text { Jan. ' } 57\end{array}$

$4107 \quad 7126 \quad 30$ Dec. ' 59 * No Returns

" +

"

\begin{tabular}{llllll}
41 & 09.5 & 70 & 24 & 15 \\
\hline
\end{tabular}

$\begin{array}{llllll}41 & 07 & 70 & 31 & 26 & \text { Mar. '57 }\end{array}$

No Rèturns

" "

"

" 1

it

" 1

"

11

\begin{tabular}{llllll}
45 & 12 & 64 & 23 & 9 & Dec. \\
\hline 57
\end{tabular}

* In 31 fathoms 
Sllver Bay Cruise 4 (cont'd)

Released

\begin{tabular}{|c|c|c|c|c|c|c|c|}
\hline Sta. \# & Bottle \# & \multicolumn{2}{|c|}{ Lat. } & \multicolumn{2}{|c|}{ Long. } & \multicolumn{2}{|c|}{ Date } \\
\hline 154 & & 42 & 30 & 69 & 29 & 25 & Nov. \\
\hline 156 & & 42 & 10.5 & 69 & 29.5 & 25 & $"$ \\
\hline 162 & & 42 & 10 & 68 & 28.5 & 27 & $"$ \\
\hline 165 & & 41 & 59.8 & 68 & 03 & 27 & $"$ \\
\hline 169 & & 42 & 18.2 & 67 & 35.5 & 27 & $"$ \\
\hline 172 & & 42 & 07.5 & 66 & 58.5 & 27 & $"$ \\
\hline 176 & & 42 & 22.3 & 66 & 34.8 & 27 & $"$ \\
\hline 180 & & 42 & 04.5 & 65 & 52 & 27 & $"$ \\
\hline 181 & & 42 & 03 & 65 & 36.5 & 27 & $"$ \\
\hline 183 & & 41 & 48.2 & 65 & 36.5 & 28 & $"$ \\
\hline 196 & & 41 & 44.5 & 66 & 55 & 28 & $"$ \\
\hline 200 & & 41 & 12.2 & 68 & 00 & 28 & $"$ \\
\hline 206 & & 41 & 16.5 & 69 & 01 & 29 & $"$ \\
\hline 207 & & 41 & 17.5 & 69 & 19.5 & 29 & $"$ \\
\hline 211 & & 40 & 51.5 & 69 & 05.8 & 29 & $"$ \\
\hline 216 & & 41 & 27.5 & 68 & 41.2 & 29 & $"$ \\
\hline 221 & & 41 & 29.5 & 69 & 32.5 & 29 & $"$ \\
\hline 224 & 27549 & 41 & 45.5 & 69 & 04 & 29 & $"$ \\
\hline 230 & & 41 & 52.5 & 67 & 59 & 30 & $"$ \\
\hline
\end{tabular}

Recovered

Lat. Long. Date

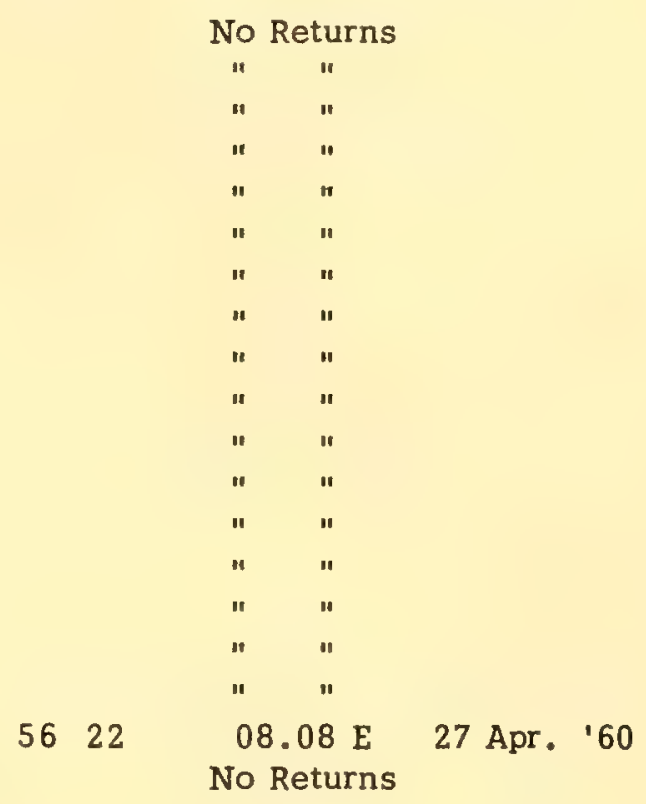




\section{November - 8 December}

1956

Stations 1-35, 67-74, P, Q, R, S, T, U; 6 bottles dropped, all ballasted.

Stations 36-66; 12 bottles dropped, even numbered bottles ballasted, odd numbered bottles not ballasted.

\section{Released}

\begin{tabular}{|c|c|c|c|c|c|c|c|}
\hline Sta.\# & Bottle \# & \multicolumn{2}{|c|}{ Lat. } & \multicolumn{2}{|c|}{ Long. } & \multicolumn{2}{|c|}{ Date } \\
\hline 1 & 25419 & 44 & 59.5 & 66 & 49.2 & 14 & Nov. \\
\hline 2 & & 45 & 01.2 & 66 & 26.5 & 14 & $"$ \\
\hline 3 & & 44 & 58.5 & 66 & 22.5 & 14 & " \\
\hline 4 & & 44 & 55.5 & 66 & 17.8 & 14 & $"$ \\
\hline 5 & & 44 & 52.5 & 66 & 13.0 & 14 & $"$ \\
\hline 6 & & 44 & 49.5 & 66 & 08.0 & 14 & $"$ \\
\hline 7 & & 44 & 46.5 & 66 & 03.5 & 14 & $"$ \\
\hline 8 & & 44 & 43.5 & 65 & 58.8 & 15 & $"$ \\
\hline 9 & & 44 & $40: 2$ & 65 & 54.0 & 15 & $"$ \\
\hline 10 & & 44 & 51.0 & 65 & 35.0 & 15 & $"$ \\
\hline 11 & 25495 & 44 & 54.3 & 65 & 38.5 & 15 & $"$ \\
\hline 12 & & 44 & 57.5 & 65 & 42.2 & 15 & $"$ \\
\hline 13 & & 45 & 00.2 & 65 & 46.0 & 15 & $"$ \\
\hline 14 & & 45 & 04.0 & 65 & 49.8 & 15 & $"$ \\
\hline 15 & 25500 & 45 & 07.0 & 65 & 53.2 & 15 & $"$ \\
\hline 16 & & 45 & 10.0 & 65 & 57.0 & 15 & $"$ \\
\hline 17 & & 45 & 15.5 & 65 & 37.0 & 15 & $"$ \\
\hline 18 & & 45 & 12.5 & 65 & 33.0 & 15 & $"$ \\
\hline 19 & & 45 & 09.0 & 65 & 29.0 & 15 & $"$ \\
\hline 20 & & 45 & 05.8 & 65 & 25.2 & 15 & $"$ \\
\hline 21 & & 45 & 02.5 & 65 & 21.2 & 15 & $"$ \\
\hline 22 & 25543 & 44 & 59.2 & 65 & 17.5 & 15 & $"$ \\
\hline 23 & & 45 & 05.8 & 65 & 03.0 & 15 & " \\
\hline 24 & & 45 & 09.2 & 65 & 07.0 & 15 & " \\
\hline 25 & & 45 & 12.5 & 65 & 11.0 & 15 & $"$ \\
\hline 26 & & 45 & 15.8 & 65 & 15.0 & 15 & " \\
\hline 27 & & 45 & 19.0 & 65 & 19.0 & 15 & $"$ \\
\hline
\end{tabular}

Recovered

Lat. Long. Date

$44 \quad 55.9 \quad 66 \quad 54.4 \quad 19$ Nov. '56 No Returns

$\begin{array}{lllll}45 & 10 & 64 & 46 & 21 \text { Dec. } 56\end{array}$ No Returns

" "

$\begin{array}{lllll}45 & 03 & 65 & 04 & 22 \mathrm{Dec} \cdot{ }^{\prime} 56\end{array}$ No Returns

4456
4 Dec. '56 
Harengus Cruise AH7 (cont'd)

Released

\begin{tabular}{|c|c|c|c|c|c|c|c|}
\hline Sta.\# & Bottle \# & & at. & & ng. & & ate \\
\hline 28 & 25575 & 45 & 22.2 & 65 & 22.7 & 15 & Nov \\
\hline 29 & & 45 & 21.8 & 65 & 17.0 & 15 & $"$ \\
\hline 30 & 25589 & 45 & 21.2 & 65 & 11 & 15 & $"$ \\
\hline 31 & & 45 & 20.8 & 65 & 05.2 & 15 & $"$ \\
\hline 32 & & 45 & 20.2 & 64 & 59.5 & 15 & $"$ \\
\hline 33 & & 45 & 16.2 & 64 & 58.0 & 15 & $"$ \\
\hline 34 & 25612 & 45 & 12.5 & 64 & 56.5 & 15 & $"$ \\
\hline 35 & 25618 & 45 & 08.5 & 64 & 55.2 & 15 & $"$ \\
\hline 74 & & 44 & 18.0 & 66 & 20.5 & 16 & $"$ \\
\hline 73 & & 44 & 24.2 & 66 & 22.3 & 16 & $"$ \\
\hline 72 & & 44 & 29.4 & 66 & 23.3 & 16 & $"$ \\
\hline 71 & & 44 & 35.0 & 66 & 24.8 & 16 & $"$ \\
\hline 70 & & 44 & 44.4 & 66 & 26.0 & 16 & $"$ \\
\hline 69 & 25653 & 44 & 45.6 & 66 & 27.0 & 16 & $"$ \\
\hline & 25657 & 44 & 45.6 & 66 & 27.0 & 16 & $"$ \\
\hline 68 & 25660 & 44 & 50.6 & 66 & 28.0 & 16 & $"$ \\
\hline 67 & 25665 & 44 & 55.8 & 66 & 29.8 & 16 & $"$ \\
\hline 36 & & 44 & 18.0 & 66 & 43.0 & 16 & $"$ \\
\hline 37 & 25687 & 44 & 07 & 66 & 21 & 16 & $"$ \\
\hline 38 & & 43 & 51.0 & 66 & 45.0 & 19 & $"$ \\
\hline 39 & 25715 & 43 & 40.0 & 66 & 12.0 & 20 & $"$ \\
\hline 40 & 25725 & 43 & 18.0 & 66 & 22.0 & 20 & $"$ \\
\hline 41 & & 43 & 18.0 & 65 & 16.0 & 20 & $"$ \\
\hline 42 & & 43 & 00.0 & 65 & 16.0 & 21 & $"$ \\
\hline 43 & 25763 & 43 & 00.0 & 66 & 31.5 & 21 & $"$ \\
\hline 44 & & 43 & 41.0 & 67 & 00.0 & 22 & $"$ \\
\hline 64 & & 44 & 31.5 & 67 & 11.5 & 27 & $"$ \\
\hline 62 & 15761 & 44 & 23.5 & 67 & 37 & 27 & $"$ \\
\hline & 15763 & 44 & 23.5 & 67 & 37 & 27 & $"$ \\
\hline 61 & & 44 & 00.0 & 67 & 37.0 & 27 & $"$ \\
\hline 60 & & 44 & 00.0 & 68 & 34.0 & 27 & " \\
\hline 59 & & 43 & 41.5 & 68 & 34.0 & 28 & $"$ \\
\hline 58 & & 43 & 41.5 & 69 & 00.0 & 28 & $"$ \\
\hline 57 & & 43 & 22.5 & 69 & 00.0 & 28 & $"$ \\
\hline 56 & & 43 & 22.5 & 70 & 20.0 & 28 & $"$ \\
\hline 55 & & 43 & 04.5 & 70 & 00.0 & 28 & $"$ \\
\hline
\end{tabular}

Recovered

Lat. Long. Date
$\begin{array}{llllll}44 & 56.7 & 65 & 16.5 & 20 & \text { Dec. } \\ & 56\end{array}$ No Returns
$\begin{array}{lllll}45 & 17 & 64 & 20 & 17 \\ \text { Dec. } & 56\end{array}$
No Returns
" "

$\begin{array}{lllll}45 & 19.6 & 64 & 46.5 & 24 \text { Nov. '56 }\end{array}$

$\begin{array}{lllll}45 & 22 & 64 & 20 & 20 \\ & \text { May '57 }\end{array}$

No Returns $\begin{array}{llllll}44 & 42 & 66 & 43 & 15 & \text { Sept. } \\ & 57\end{array}$

$\begin{array}{lllll}44 & 55.2 & 65 & 19 & 22 \mathrm{Dec} . \\ 4 & 56\end{array}$

$\begin{array}{lllll}44 & 44.6 & 66 & 50.6 & 19 \\ \text { Nov. '56 }\end{array}$

$45 \quad 05.3 \quad 66 \cdot 28.2 \quad 24$ Nov. '56

No Returns

$\begin{array}{lllll}44 & 26.4 & 66 & 01 & 25 \\ \text { Nov. } & 56\end{array}$

No Returns

$\begin{array}{llllll}43 & 48.8 & 66 & 08.7 & 22 \\ & \text { Nov. '56 }\end{array}$

$\begin{array}{llllll}44 & 05 & 66 & 12.6 & 27 & \text { Nov. '56 }\end{array}$

No Returns

$\begin{array}{llllll}44 & 30 \quad 66 & 06 & 17 & \text { Oct. } & \text { '58 }\end{array}$

No Returns

$\begin{array}{lllllll}44 & 03 & 66 & 10 & 11 & \text { Apr . '57 }\end{array}$

$\begin{array}{lllll}43 \quad 48 & 66 & 10 & 14 \mathrm{Feb} \cdot & 157\end{array}$
No Returns

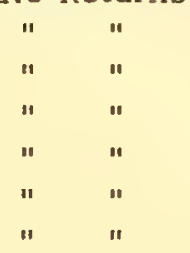


Harengus Cruise AH7 (cont'd)

Released

\begin{tabular}{|c|c|c|c|c|c|c|c|}
\hline Sta.\# & Bottle \# & \multicolumn{2}{|c|}{ Lat. } & \multicolumn{2}{|c|}{ Long. } & \multicolumn{2}{|c|}{ Date } \\
\hline 53 & & 42 & 42.5 & 70 & 00.0 & 28 & Nov. \\
\hline 52 & & 42 & 20 & 70 & 39 & 28 & $"$ \\
\hline \multirow[t]{3}{*}{51} & 15876 & 41 & 55.5 & 70 & 27 & 29 & $"$ \\
\hline & 15879 & 41 & 55.5 & 70 & 27 & 29 & $"$ \\
\hline & 15882 & 41 & 55.5 & 70 & 27 & 29 & $"$ \\
\hline 50 & & 42 & 39.5 & 69 & 31.5 & 29 & $"$ \\
\hline 49 & & 43 & 00.0 & 69 & 31.5 & 29 & $"$ \\
\hline 48 & & 43 & 00.0 & 68 & 33.0 & 30 & $"$ \\
\hline 47 & & 43 & 22.0 & 68 & 33.0 & 30 & $"$ \\
\hline 46 & 25405 & 43 & 22.0 & 68 & 00.0 & 30 & $"$ \\
\hline 45 & 24731 & 43 & 41.0 & 68 & 00.0 & 30 & $"$ \\
\hline 63 & 24743 & 44 & 00.0 & 67 & 00.0 & 30 & $"$ \\
\hline 65 & & 44 & 53.8 & 66 & 45.0 & 30 & $"$ \\
\hline 66 & & 44 & 57.8 & 66 & 51.0 & 1 & Dec. \\
\hline$Q$ & & 45 & 05.4 & 65 & 53.2 & 4 & $"$ \\
\hline \multirow[t]{2}{*}{$\mathrm{P}$} & 28308 & 45 & 10.0 & 65 & 57.5 & 5 & $"$ \\
\hline & 28309 & 45 & 10.0 & 65 & 57.5 & 5 & $"$ \\
\hline S & & 44 & 58.4 & 65 & 44.2 & 5 & $"$ \\
\hline$T$ & & 45 & 08.5 & 65 & 51.4 & 7 & $"$ \\
\hline U & & 44 & 59.2 & 66 & 02.6 & 8 & $"$ \\
\hline
\end{tabular}

Recovered

$$
\text { Lat. Long. Date }
$$

No Returns

$4200 \quad 70 \quad 05 \quad 6$ Dec. 56

$\begin{array}{llllll}41 & 59.5 & .70 & 04.8 & 13 & \text { Apr. } 57\end{array}$

$\begin{array}{lllll}4152 & 70 & 00.6 & 21 & \text { Dec. '56 }\end{array}$

No Returns

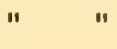

" 11

" "

$\begin{array}{llllll}43 & 48.5 & 66 & 10 & 23 \mathrm{Feb} . & \end{array}$

$\begin{array}{lllll}44 & 09 & 66 & 12 & 25 \\ \text { Jan. } & 57\end{array}$

$\begin{array}{llllll}41 & 14: 5 & 70 & 03 & 4 & \mathrm{Jul}\end{array}$

No Returns

" "

$\begin{array}{lllll}44 & 15.3 & 66 & 20.6 & 5 \\ \text { Jan. } & 57\end{array}$

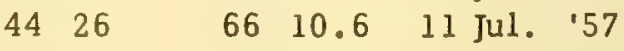

No Returns

" $"$ 


\section{Delaware Cruise 57-1}

26 January - 4 February

\section{7}

12 ballasted bottles released at each station.

Released

\begin{tabular}{|c|c|c|c|c|c|c|c|}
\hline Sta.\# & Bottle \# & \multicolumn{2}{|c|}{ Lat. } & \multicolumn{2}{|c|}{ Long. } & \multicolumn{2}{|c|}{ Date } \\
\hline 1 & & 42 & 31.5 & 68 & 56.5 & 26 & Jan. \\
\hline 2 & & 42 & 27.5 & 67 & 59 & 26 & $"$ \\
\hline 4 & & 42 & 32 & 67 & 02 & 26 & $"$ \\
\hline 6 & & 43 & 03 & 66 & 59 & 26 & $"$ \\
\hline 10 & 27897 & 43 & 13 & 67 & 50 & 27 & $"$ \\
\hline 12 & & 43 & 22 & 68 & 15 & 27 & $"$ \\
\hline 15 & & 43 & 24 & 68 & 37 & 27 & $"$ \\
\hline 20 & & 43 & 26.5 & 69 & 32 & 27 & $"$ \\
\hline 22 & & 43 & 28.5 & 69 & 57 & 27 & $"$ \\
\hline 27 & 27956 & 43 & 08 & 70 & 20.5 & 28 & $"$ \\
\hline 29 & & 42 & 58 & 69 & 54.5 & 28 & $"$ \\
\hline 33 & & 42 & 53 & 69 & 00 & 29 & $"$ \\
\hline 39 & & 42 & 47 & 67 & 49 & 29 & $"$ \\
\hline 44 & & 42 & 42 & 66 & 58 & 29 & $"$ \\
\hline 53 & & 42 & 35.5 & 65 & 54 & 29 & $"$ \\
\hline 58 & & 42 & 28.5 & 65 & 02 & 30 & $"$ \\
\hline 64 & & 43 & 13 & 65 & 47.5 & 30 & $"$ \\
\hline 66 & 28050 & 43 & 00 & 66 & 14 & 30 & $"$ \\
\hline 70 & & 42 & 36.5 & 66 & 29 & 30 & $"$ \\
\hline 76 & & 41 & 46.5 & 67 & 05.5 & 30 & $"$ \\
\hline 79 & & 41 & 20 & 67 & 17 & 31 & $"$ \\
\hline 83 & & 40 & 44 & 67 & 40 & 31 & $"$ \\
\hline 87 & & 40 & 11 & 67 & 53 & 31 & $"$ \\
\hline 90 & & 40 & 00 & 68 & 30 & 31 & " \\
\hline 92 & & 39 & 58 & 69 & 00 & 31 & $"$ \\
\hline 95 & & 39 & 57.5 & 69 & 28.5 & 31 & $"$ \\
\hline 97 & & 40 & 00 & 70 & 00 & 31 & $"$ \\
\hline 101 & & 40 & 37 & 70 & 02 & 31 & " \\
\hline 106 & & 40 & 39 & 68 & 55 & 1 & Feb \\
\hline 1? 1 & & 40 & 40 & 68 & 05 & 1 & $"$ \\
\hline
\end{tabular}

Recovered

Lat. Long. Date

No Returns

"

11

11

\begin{tabular}{llllll}
41 & 37.5 & 70 & 25 & 6 & Oct. \\
\hline & 57
\end{tabular}

No Returns

" 1

"

"

$\begin{array}{llllll}41 & 48.4 & 69 & 57.4 & 8 & \text { Jan. '58 }\end{array}$ No Returns

$$
\begin{array}{ll}
\text { " } & \text { " } \\
\text { " } & \text { " } \\
\text { " } & 11 \\
\text { " } & \text { " }
\end{array}
$$

$\begin{array}{lllllll}44 & 11.7 & 66 & 09.8 & 31 & \text { May } & 57\end{array}$ No Returns

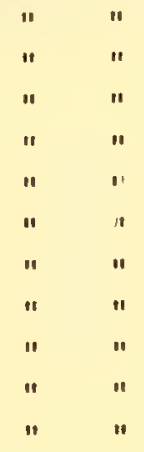


Released

\begin{tabular}{|c|c|c|c|c|c|c|c|}
\hline Sta.\# & Bottle \# & & at. & & ng. & & te \\
\hline 116 & & 40 & 40 & 66 & 54 & 1 & $\mathrm{Feb}$. \\
\hline 121 & & 41 & 14 & 66 & 21 & 1 & $"$ \\
\hline 129 & & 41 & 14.5 & 68 & 00.5 & 2 & " \\
\hline 133 & & 41 & 13.5 & 68 & 54 & 2 & " \\
\hline 135 & & 41 & 12.5 & 69 & 16 & 2 & $"$ \\
\hline 137 & & 41 & 05 & 69 & 06 & 2 & $"$ \\
\hline 141 & 28280 & 41 & 27 & 68 & 39.5 & 2 & " \\
\hline 146 & & 41 & 36 & 69 & 35 & 2 & $"$ \\
\hline 149 & & 41 & 45.5 & 69 & 02.5 & 2 & $"$ \\
\hline 154 & & 41 & 48.5 & 67 & 58.5 & 3 & " \\
\hline 163 & & 41 & 47 & 65 & 58 & 3 & $"$ \\
\hline 165 & & 41 & 46 & 65 & 41.5 & 3 & " \\
\hline 167 & & 42 & 09.5 & 65 & 36.5 & 3 & $"$ \\
\hline 169 & & 42 & 06 & 66 & 00 & 3 & $"$ \\
\hline 171 & & 42 & 19.5 & 66 & 30.5 & 3 & $"$. \\
\hline 175 & & 42 & 04.5 & 66 & 58 & 4 & $"$ \\
\hline 178 & & 42 & 20 & 67 & 32.5 & 4 & $"$ \\
\hline 182 & & 41 & 57.5 & 67 & 59 & 4 & $"$ \\
\hline 184 & & 42 & 10 & 68 & 30 & 4 & " \\
\hline 189 & & 42 & 08.5 & 69 & 27 & 4 & $"$ \\
\hline 193 & & 42 & 20 & 70 & 07 & 4 & $"$ \\
\hline 194 & & 42 & 21.5 & 70 & 23.5 & 4 & $"$ \\
\hline 196 & 28511 & 42 & 21 & 70 & 41 & 4 & " \\
\hline & 28514 & 42 & 21 & 70 & 41 & 4 & $"$ \\
\hline & 28517 & 42 & 21 & 70 & 41 & 4 & $"$ \\
\hline & 28518 & 42 & 21 & 70 & 41 & 4 & $"$ \\
\hline
\end{tabular}

Recovered

Lat. Long. Date

No Returns



$\begin{array}{lllllll}66 & 00 & 12 & 01 \mathrm{E} & 29 & \text { Oct. '59 }\end{array}$ No Returns

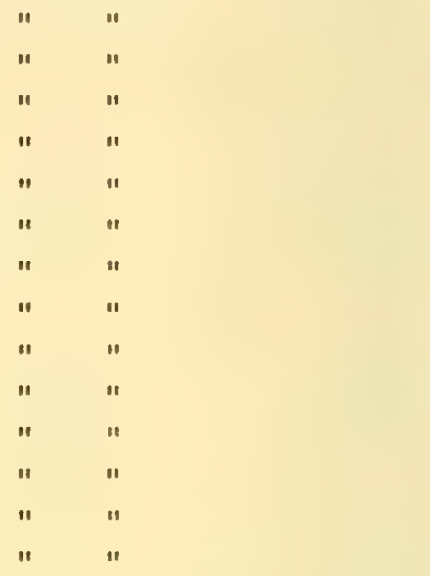

$\begin{array}{llll}41 & 48 & 70 & 00.7 \mathrm{~W} \\ 28 & \text { Apr. } 57\end{array}$

$\begin{array}{llllll}42 & 01.8 & 70 & 37.5 & 7 \text { Apr. } & \end{array}$

$\begin{array}{llllll}42 & 01 & 70 & 05 & 9 & \text { Mar. '57 }\end{array}$

$\begin{array}{llllll}41 & 49 & 70 & 00 & 26 & \text { Oct. } 157\end{array}$ 
Albatross III Cruise 88

$$
19-28 \text { February }
$$

1957

12 ballasted bottles released at each station.

\section{Released}

\begin{tabular}{|c|c|c|c|c|c|c|c|}
\hline Sta.\# & Bottle \# & & at. & & ng. & & \\
\hline 7 & & 40 & 49.4 & 72 & 00 & 19 & eb. \\
\hline 13 & & 40 & 00 & 72 & 00 & 20 & " \\
\hline 20 & & 40 & 00 & 71 & 00.5 & 20 & $"$ \\
\hline 26 & & 40 & 00 & 70 & 00 & 20 & $"$ \\
\hline 35 & & 40 & 59 & 69 & 05 & 21 & $"$ \\
\hline 48 & 28527 & 42 & 08 & 70 & 05.5 & 21 & $"$ \\
\hline 52 & 28532 & 41 & 49.7 & 70 & 27.7 & 21 & $"$ \\
\hline & 28535 & 41 & 49.7 & 70 & 27.7 & 21 & " \\
\hline & 28537 & 41 & 49.7 & 70 & 27.7 & 21 & $"$ \\
\hline & 28538 & 41 & 49.7 & 70 & 27.7 & 21 & $"$ \\
\hline & 28539 & 41 & 49.7 & 70 & 27.7 & 21 & $"$ \\
\hline & 28540 & 41 & 49.7 & 70 & 27.7 & 21 & $"$ \\
\hline & 28542 & 41 & 49.7 & 70 & 27.7 & 21 & " \\
\hline 59 & 28547 & 42 & 38 & 70 & 30 & 22 & $"$ \\
\hline & 28549 & 42 & 38 & 70 & 30 & 22 & $"$ \\
\hline 64 & & 43 & 06.7 & 70 & 23 & 22 & $"$ \\
\hline 70 & 28568 & 43 & 40 & 69 & 45 & 22 & " \\
\hline & 28573 & 43 & 40 & 69 & 45 & 22 & $"$ \\
\hline 78 & & 43 & 59 & 68 & 20 & 22 & $"$ \\
\hline 83 & & 44 & 22.5 & 67 & 37 & 23 & $"$ \\
\hline 88 & 19775 & 44 & 46.5 & 66 & 53 & 23 & $"$ \\
\hline 94 & & 45 & 09.5 & 65 & 51.5 & 23 & $"$ \\
\hline 101 & & 45 & 34 & 64 & 46 & 23 & " \\
\hline 105 & 19863 & 45 & 05 & 65 & 15 & 24 & $"$ \\
\hline & 19878 & 45 & 05 & 65 & 15 & 24 & $"$ \\
\hline 121 & 23954 & 43 & 40.5 & 66 & 20.5 & 24 & $"$ \\
\hline & 23955 & 43 & 40.5 & 66 & 20.5 & 24 & " \\
\hline
\end{tabular}

\section{Recovered}

Lat. Long. Date

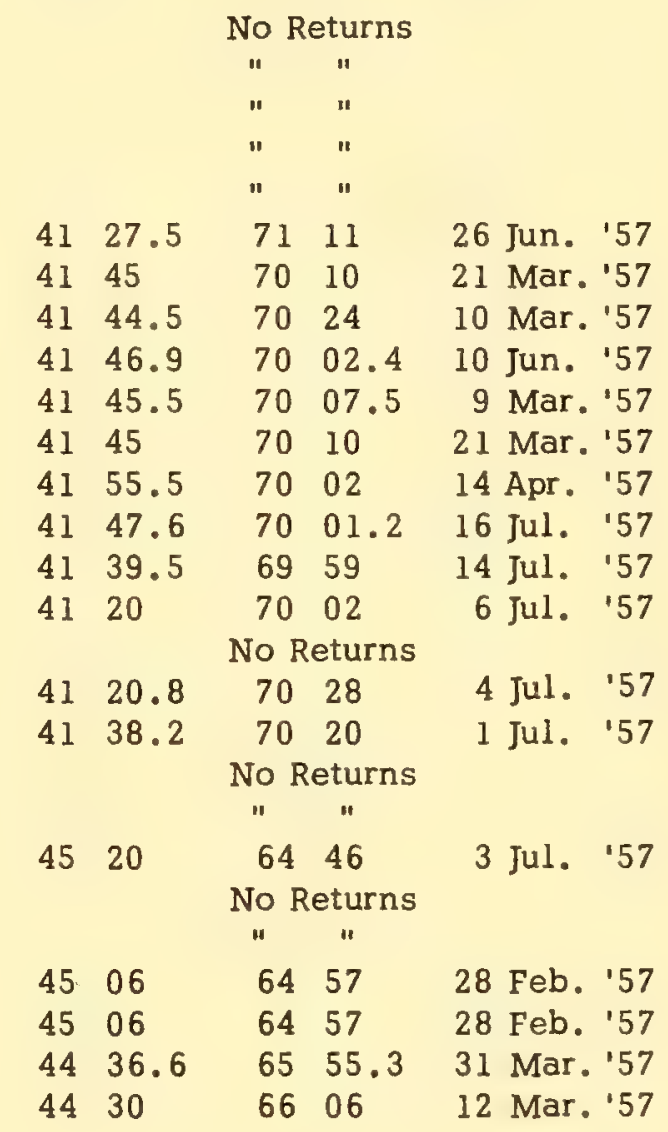


Released

\begin{tabular}{|c|c|c|c|c|c|c|c|c|c|c|c|c|}
\hline \multirow{2}{*}{$\begin{array}{l}\text { Sta.\# } \\
125\end{array}$} & \multirow{2}{*}{$\begin{array}{c}\text { Bottle \# } \\
23969\end{array}$} & \multicolumn{2}{|c|}{ Lat. } & \multicolumn{2}{|c|}{ Long . } & \multicolumn{2}{|c|}{ Date } & \multicolumn{2}{|c|}{ Lat. } & \multicolumn{2}{|c|}{ Long. } & \multirow{2}{*}{$\begin{array}{l}\text { Date } \\
20 \text { Sept.'57 }\end{array}$} \\
\hline & & 44 & 15 & 66 & 18 & 24 & Feb. & 45 & 41.7 & 64 & 27.3 & \\
\hline 132 & 24018 & 44 & 05.3 & 66 & 28 & 25 & $"$ & 44 & 29.8 & 66 & 06.1 & 22 Mar. '57 \\
\hline 137 & & 44 & 28.7 & 67 & 10.5 & 25 & $"$ & & & No $R$ & eturns & \\
\hline \multirow[t]{4}{*}{141} & 23989 & 43 & 49 & 66 & 55.5 & 25 & $"$ & 43 & 41.5 & 66 & 02 & 14 Apr. '57 \\
\hline & 23995 & 43 & 49 & 66 & 55.5 & 25 & $"$ & 44 & 33 & 66 & 02 & $12 \mathrm{Apr} . \quad 57$ \\
\hline & 23998 & 43 & 49 & 66 & 55.5 & 25 & $"$ & 45 & 17 & 64 & 47 & 3 Jul. $\quad 57$ \\
\hline & 23999 & 43 & 49 & 66 & 55.5 & 25 & $"$ & 44 & 17.5 & 66 & 19 & $24 \mathrm{Mar}$. 57 \\
\hline 156 & 24010 & 43 & 39 & 68 & 35 & 26 & $"$ & 45 & 03 & 66 & 48.5 & 27 Oct. '57 \\
\hline 161 & 29611 & 43 & 25 & 69 & 01.5 & 26 & $"$ & 44 & 30 & 66 & 06 & 28 Sept.' 57 \\
\hline \multirow[t]{4}{*}{165} & 29616 & 43 & 22 & 69 & 53.5 & 26 & $"$ & 41 & 19.4 & 70 & 45.4 & 13 Sept.'57 \\
\hline & 29618 & 43 & 22 & 69 & 53.5 & 26 & $"$ & 41 & 15.7 & 70 & 49 & $11 \mathrm{Jul} . \quad 57$ \\
\hline & 29619 & 43 & 22 & 69 & 53.5 & 26 & $"$ & 41 & 36.5 & 69 & 59.4 & 22 Nov. '57 \\
\hline & 29626 & 43 & 22 & 69 & 53.5 & 26 & $"$ & 41 & 20.8 & 70 & 30 & 23 Sept.'57 \\
\hline 169 & 29629 & 43 & 00.5 & 70 & 05 & 26 & $"$ & 41 & 54 & 69 & 58 & 18 Mar. 57 \\
\hline \multirow{7}{*}{174} & 29640 & 42 & 19.5 & 70 & 22 & 26 & $"$ & 42 & 04 & 70 & 14 & 9 Mar. '57 \\
\hline & 29641 & 42 & 19.5 & 70 & 22 & 26 & $"$ & 35 & 50 & 75 & 33 & 15 Oct. '57 \\
\hline & 29642 & 42 & 19.5 & 70 & 22 & 26 & $"$ & 42 & 04 & 70 & 14.5 & 23 Mar. '57 \\
\hline & 29644 & 42 & 19.5 & 70 & 22 & 26 & $"$ & 42 & 04 & 70 & 14 & 9 Mar. '57 \\
\hline & 29646 & 42 & 19.5 & 70 & 22 & 26 & $"$ & 42 & 04 & 70 & 14 & 9 Mar. '57 \\
\hline & 29650 & 42 & 19.5 & 70 & 22 & 26 & $"$ & 41 & 21.5 & 70 & 28.3 & 7 Aug. ' 57 \\
\hline & 29651 & 42 & 19.5 & 70 & 22 & 26 & $"$ & 42 & 04 & 70 & 14 & 9 Mar. '57 \\
\hline 181 & & 42 & 01 & 69 & 13.5 & 27 & $"$ & \multicolumn{5}{|c|}{ No Returns } \\
\hline 187 & & 41 & 34 & 68 & 29 & 27 & $"$ & & & $"$ & $n$ & \\
\hline \multirow[t]{3}{*}{191} & 20008 & 44 & 44.5 & 65 & 47 & 24 & $"$ & 44 & 40.5 & 65 & 48.6 & $25 \mathrm{Feb} . ' 57$ \\
\hline & 20010 & 44 & 44.5 & 65 & 47 & 24 & $"$ & 44 & 40.5 & 65 & 48.6 & 27 Feb. '57 \\
\hline & 20011 & 44 & 44.5 & 65 & 47 & 24 & $"$ & 44 & 37 & 65 & 41.5 & 30 Mar. '57 \\
\hline 195 & & 40 & 39 & 68 & 53 & 27 & $"$ & \multicolumn{5}{|c|}{ No Returns } \\
\hline 202 & & 40 & 40 & 70 & 00 & 28 & $"$ & & & $"$ & $"$ & \\
\hline 205 & & 40 & 40 & 70 & 35 & 28 & $"$ & & & $"$ & $"$ & \\
\hline
\end{tabular}




\title{
Albatross III Cruise 90
}

\author{
11 - 17 April
}

1957

12 bottles released at each station, odd numbers - ballasted, even numbers - no ballast.

Released

\begin{tabular}{|c|c|c|c|c|c|c|c|}
\hline Sta.\# & Bottle \# & & at. & & ng. & & ate \\
\hline 1 & 29719 & 41 & 36 & 69 & 49.5 & 11 & Apr . \\
\hline & 29721 & 41 & 36 & 69 & 49.5 & 11 & $"$ \\
\hline & 29722 & 41 & 36 & 69 & 49.5 & 11 & $"$ \\
\hline & 29723 & 41 & 36 & 69 & 49.5 & 11 & $"$ \\
\hline 2 & & 41 & 43.5 & 69 & 24 & 11 & $"$ \\
\hline 3 & 29740 & 41 & 50.5 & 68 & 59 & 11 & $"$ \\
\hline 4 & & 41 & 56 & 68 & 40 & 11 & $"$ \\
\hline 5 & 29760 & 42 & 05.5 & 68 & 11 & 11 & $"$ \\
\hline 6 & & 42 & 08.5 & 67 & 39.5 & 12 & $"$ \\
\hline 7 & & 42 & 10 & 67 & 14 & 12 & $"$ \\
\hline 8 & 29801 & 42 & 12 & 66 & 48.5 & 12 & $"$ \\
\hline 9 & & 42 & 13.5 & 66 & 19 & 12 & $"$ \\
\hline 10 & & 42 & 08.3 & 65 & 23.9 & 12 & $"$ \\
\hline 11 & & 42 & 01 & 65 & 24 & 12 & $"$ \\
\hline 12 & & 41 & 55.5 & 65 & 26 & 12 & $"$ \\
\hline 13 & & 41 & 54 & 65 & 50 & 12 & $"$ \\
\hline 14 & 29870 & 41 & 56 & 66 & 19 & 12 & $"$ \\
\hline & 29877 & 41 & 56 & 66 & 19 & 12 & $"$ \\
\hline 15 & & 42 & 01 & 66 & 47 & 12 & $"$ \\
\hline 16 & & 41 & 55.8 & 67 & 17.2 & 13 & $"$ \\
\hline 17 & 29912 & 41 & 54 & 67 & 36.8 & 13 & $"$ \\
\hline & 29913 & 41 & 54 & 67 & 36.8 & 13 & $"$ \\
\hline 18 & & 41 & 38.7 & 67 & 36 & 13 & $"$ \\
\hline 19 & 29938 & 41 & 39 & 67 & 13.5 & 13 & $"$ \\
\hline 20 & 29951 & 41 & 42.5 & 66 & 45 & 13 & $"$ \\
\hline 21 & & 41 & 42.2 & 66 & 16 & 13 & $"$ \\
\hline 22 & & 41 & 39.3 & 65 & 49 & 13 & $"$ \\
\hline 23 & & 41 & 37.8 & 65 & 22.7 & 13 & $"$ \\
\hline 24 & & 41 & 21.3 & 65 & 24 & 13 & $"$ \\
\hline 25 & & 41 & 21.5 & 65 & 50 & 13 & $"$ \\
\hline
\end{tabular}

\section{Recovered}

Lat. Long. Date

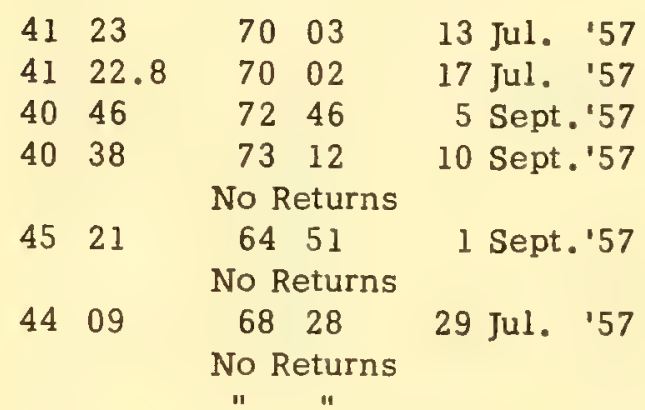

$45 \quad 20$

$64 \quad 46$

3 Jul. '57

$$
\begin{array}{ll}
\text { No Returns } & \text { " } \\
\text { " } & \text { " } \\
\text { " } & \text { " }
\end{array}
$$

$\begin{array}{llllll}41 & 20 & 70 & 49.5 & 26 \mathrm{Jul} . & \end{array}$

$\begin{array}{llllll}41 & 20.8 & 70 & 30.6 & \text { Jul. '57 }\end{array}$ No Returns

$\begin{array}{llllll}41 & 20.8 & 70 & 30 & 8 & \text { Dec. } 57\end{array}$

$\begin{array}{llllll}41 & 21.1 & 70 & 46.3 & 24 & \text { Sept.'57 }\end{array}$ No Returns

$\begin{array}{llllll}41 & 36.4 & 70 & 24 & 29 & \text { Jun. ' } 57\end{array}$

$\begin{array}{llllll}41 & 21 & 70 & 02 & 13 & \text { Aug. '57 }\end{array}$ 
Released

\begin{tabular}{|c|c|c|c|c|c|c|c|}
\hline \multirow{2}{*}{$\begin{array}{c}\text { Sta.\# } \\
26\end{array}$} & \multirow[t]{2}{*}{ Bottle \# } & \multicolumn{2}{|c|}{ Lat. } & \multicolumn{2}{|c|}{ Long. } & \multicolumn{2}{|c|}{ Date } \\
\hline & & 41 & 23 & 66 & 15 & 13 & Apr \\
\hline 27 & 30034 & 41 & 23 & 66 & 37.5 & 13 & $"$ \\
\hline 28 & & 41 & 21 & 66 & 45.3 & 14 & $"$ \\
\hline 29 & 30048 & 41 & 19 & 67 & 13 & 14 & $"$ \\
\hline 30 & 30098 & 41 & 18.5 & 67 & 51 & 14 & $"$ \\
\hline 31 & 30076 & 41 & 00.8 & 67 & 51.8 & 14 & $"$ \\
\hline 32 & 30092 & 41 & 01.5 & 67 & 14 & 14 & $"$ \\
\hline 33 & & 41 & 00 & 66 & 54.5 & 14 & $"$ \\
\hline 34 & & 40 & 58 & 66 & 26.5 & 14 & $"$ \\
\hline 35 & & 40 & 58.5 & 66 & 01 & 14 & $"$ \\
\hline 36 & & 40 & 56.5 & 65 & 49 & 14 & $"$ \\
\hline 37 & & 40 & 40 & 65 & 50 & 14 & $"$ \\
\hline 38 & & 40 & 39.3 & 66 & 28.7 & 14 & $"$ \\
\hline 39 & & 40 & 40.5 & 66 & 56 & 15 & $"$ \\
\hline 40 & 30186 & 40 & 40.5 & 67 & 26 & 15 & $"$ \\
\hline 41 & & 40 & 41.5 & 67 & 48.3 & 15 & $"$ \\
\hline \multirow[t]{2}{*}{42} & 30207 & 40 & 57 & 67 & 32.5 & 15 & $"$ \\
\hline & 30210 & 40 & 57 & 67 & 32.5 & 15 & $"$ \\
\hline \multirow[t]{2}{*}{43} & 30218 & 41 & 13 & 67 & 09 & 15 & $"$ \\
\hline & 30225 & 41 & 13 & 67 & 09 & 15 & $"$ \\
\hline 44 & & 41 & 23 & 66 & 48 & 15 & $"$ \\
\hline 45 & 30250 & 41 & 33 & 66 & 35.7 & 15 & $"$ \\
\hline 46 & 30260 & 41 & 46 & 66 & 19.7 & 15 & $"$ \\
\hline 47 & & 42 & 04 & 66 & 03 & 15 & $"$ \\
\hline 48 & & 41 & 23 & 67 & 04 & 16 & $"$ \\
\hline 49 & 30288 & 41 & 23 & 67 & 33 & 16 & " \\
\hline 50 & & 41 & 28 & 68 & 16 & 16 & $"$ \\
\hline 51 & 30312 & $4 I$ & 32 & 68 & 43 & 16 & $"$ \\
\hline 52 & & 41 & 34 & 68 & 58.5 & 16 & $"$ \\
\hline \multirow[t]{3}{*}{53} & 30341 & 41 & 29 & 69 & 36 & 17 & $"$ \\
\hline & 30343 & 41 & 29 & 69 & 36 & 17 & $"$ \\
\hline & 30344 & 41 & 29 & 69 & 36 & 17 & $"$ \\
\hline \multirow[t]{5}{*}{54} & 30348 & 41 & 35.5 & 69 & 51 & 17 & $"$ \\
\hline & 30351 & 41 & 35.5 & 69 & 51 & 17 & $"$ \\
\hline & 30353 & 41 & 35.5 & 69 & 51 & 17 & $"$ \\
\hline & 30354 & 41 & 35.5 & 69 & 51 & 17 & $"$ \\
\hline & 30358 & 41 & 35.5 & 69 & 51 & 17 & $"$ \\
\hline
\end{tabular}

Recovered

Long. Date

No Returns

$41 \quad 20$

$\begin{array}{ll}70 & 49.5\end{array}$

No Returns

$\begin{array}{llllll}41 & 15.1 & 70 & 10 & 4 \text { Jul. '57 }\end{array}$

\begin{tabular}{llllll}
41 & 16.2 & 70 & 13 & 7 & $\mathrm{Jul}$ \\
\hline & 157
\end{tabular}

$\begin{array}{llllll}41 & 14.4 & 70 & 02.5 & 4 \text { Jul. } 157\end{array}$

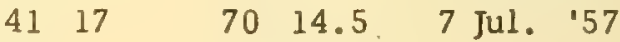

No Returns

$\begin{array}{llll}41 & 28.5 & 71 & 09\end{array}$

31 Jul. 157

No Returns

$\begin{array}{llllll}41 & 21 & 70 & 01.6 & 10 & \mathrm{Jul}\end{array}$

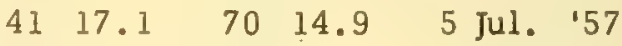

$\begin{array}{llllll}41 & 14.6 & 70 & 05 & 2 \mathrm{Jul} . & 57\end{array}$

$\begin{array}{llllll}40 & 11.5 & 74 & 00.5 & 24 & \text { Aug. } 57\end{array}$

No Returns

$\begin{array}{llllll}41 & 26 & 70 & 54 & 9 & \text { Aug. '57 }\end{array}$

$42 \quad 47$

6601

8 Jun. 157

No Returns

$\begin{array}{llllll}40 & 37.3 & 73 & 17.2 & 21 & \text { Sept. } 57\end{array}$ No Returns

$43 \quad 58$

5948

1 Oct. '57

No Returns

\begin{tabular}{llllll}
41 & 19 & 70 & 03 & 10 \\
\hline
\end{tabular}

\begin{tabular}{llllll}
41 & 14.6 & 70 & 07.7 & 25 & Jun. \\
\hline & 57
\end{tabular}

$\begin{array}{llllll}41 & 20.8 & 70 & 30.7 & 23 & \text { Jun. ' } 57\end{array}$

$4120 \quad 70 \quad 44 \quad 30$ Jun. '57

$\begin{array}{lllll}41 & 20 & 70 & 49.5 & 23 \\ \text { Jun. '57 }\end{array}$

$\begin{array}{llllll}41 & 34 & 70 & 39 & 13 & \text { Oct. ' } 57\end{array}$

$\begin{array}{llllll}41 & 19 & 71 & 49 & 29 & \text { Jul. }\end{array}$

$\begin{array}{lllll}41 & 39 & 70 & 11.6 & 27 \\ \text { Jun. '57 }\end{array}$ 
Albatross III Cruise 92

25 April - 1 May

1957

12 bottles released at each station, odd numbers - ballasted, even numbers - no ballast.

Released

.

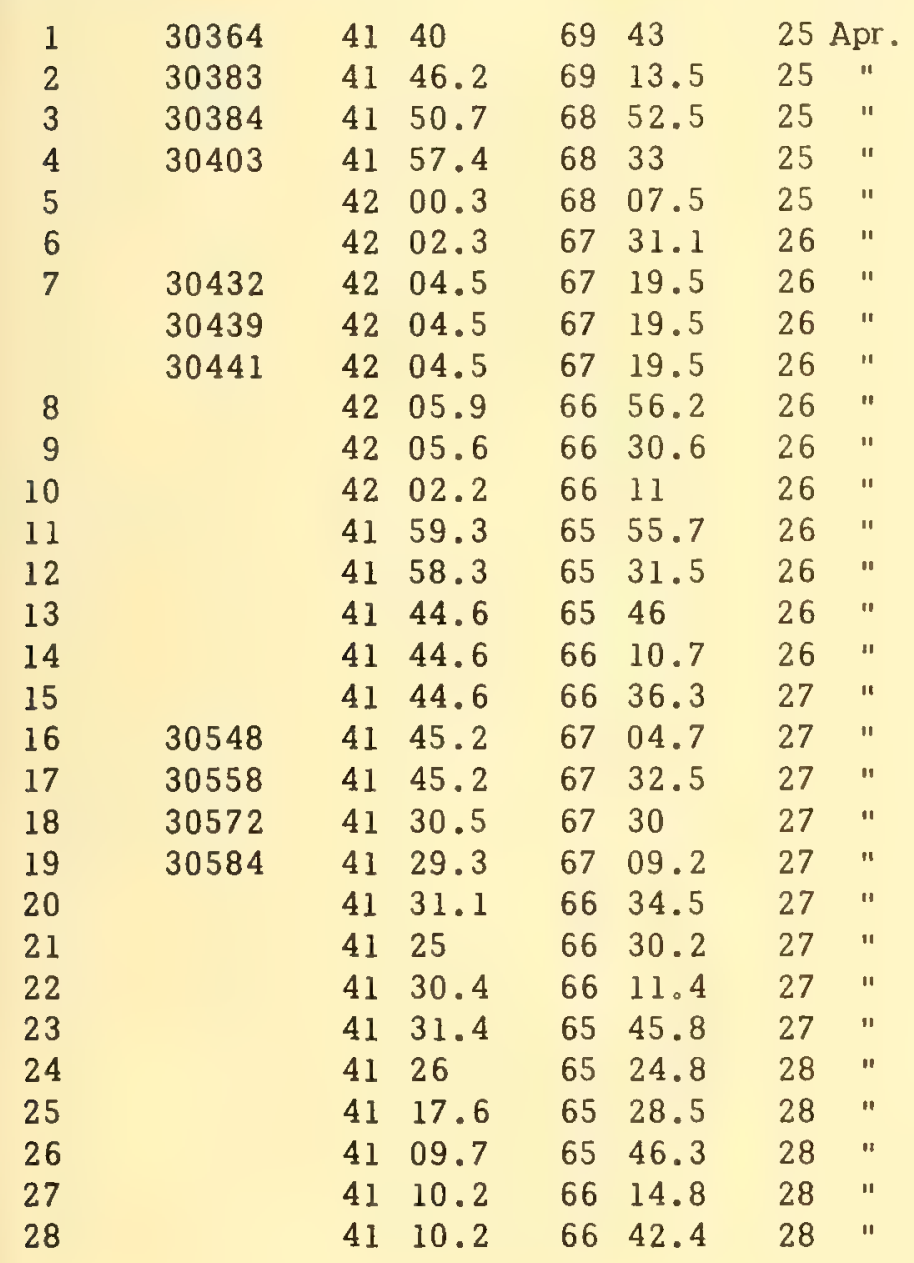

Recovered

Lat. Long. Date

\begin{tabular}{|c|c|c|}
\hline $43 \quad 32$ & $\begin{array}{ll}65 & 29.5\end{array}$ & 29 Jun. '57 \\
\hline 4130.5 & 7059 & 17 Apr. '58 \\
\hline $43 \quad 56$ & $\begin{array}{ll}60 & 03\end{array}$ & 29 Sept.' 57 \\
\hline 4140 & $70 \quad 00$ & 17 Oct.' 57 \\
\hline
\end{tabular}

$\begin{array}{lllllll}45 & 07.3 & 66 & 28.7 & 12 \mathrm{Jul} . & 157\end{array}$

\begin{tabular}{llllll}
44 & 16.2 & 66 & 20 & 17 & $\mathrm{Jun}$. \\
\hline & 57
\end{tabular}

$\begin{array}{llllll}44 & 30.5 & 65 & 55 & \text { Jul. } & 57\end{array}$

No Returns

$\begin{array}{llllll}41 & 40 & 70 & 02.5 & 28 \text { Jun. '57 }\end{array}$

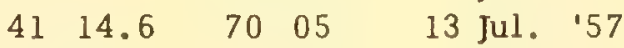

$\begin{array}{lllllll}35 & 45.5 & 75 & 30.6 & 17 & \text { Oct. } 157\end{array}$

\begin{tabular}{llllll}
41 & 29 & 71 & 09 & 29 & Jul. \\
\hline
\end{tabular}

No Returns

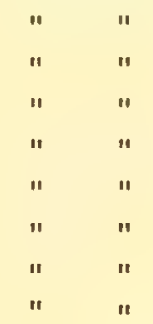


Released

\begin{tabular}{|c|c|c|c|c|c|c|c|}
\hline Sta.\# & Bottle \# & & at. & & ng. & & ate \\
\hline 29 & & 41 & 12.3 & 67 & 09.1 & 28 & $"$ \\
\hline 30 & & 41 & 11.3 & 67 & 34.5 & 28 & $"$ \\
\hline 31 & 30721 & 41 & 09.8 & 67 & 45.6 & 28 & $"$ \\
\hline 32 & 30734 & 40 & 57.2 & 67 & 59.8 & 28 & $"$ \\
\hline & 30736 & 40 & 57.2 & 67 & 59.8 & 28 & $"$ \\
\hline 33 & & 40 & 50.6 & 67 & 41.9 & 28 & $"$ \\
\hline 34 & & 40 & 52 & 67 & 18 & 28 & $"$ \\
\hline 35 & & 40 & 52.1 & 66 & 51.8 & 28 & $"$ \\
\hline 36 & 30781 & 40 & 50.7 & 66 & 25.5 & 28 & $"$ \\
\hline 37 & 30800 & 40 & 51.4 & 66 & 05.3 & 29 & $"$ \\
\hline 38 & & 40 & 50 & 65 & 42 & 29 & $"$ \\
\hline 39 & & 40 & 39 & 66 & 01.7 & 29 & $"$ \\
\hline 40 & & 40 & 33.9 & 66 & 25.2 & 29 & " \\
\hline 41 & & 40 & 31.9 & 66 & 52.4 & 29 & $"$ \\
\hline 42 & & 40 & 30.2 & 67 & 22.4 & 29 & $"$ \\
\hline 43 & & 40 & 29.5 & 67 & 43.7 & 29 & $"$ \\
\hline 44 & & 40 & 28.9 & 68 & $11 . \dot{7}$ & 29 & $"$ \\
\hline 45 & 30888 & 40 & 29.6 & 68 & 38.2 & 29 & $"$ \\
\hline & 30898 & 40 & 29.6 & 68 & 38.2 & 29 & $"$ \\
\hline 46 & & 40 & 12.2 & 68 & 41.7 & 29 & $"$ \\
\hline 47 & & 40 & 10.2 & 68 & 20.7 & 29 & $"$ \\
\hline 48 & & 40 & 11.4 & 67 & 52.2 & 29 & $"$ \\
\hline 49 & & 40 & 12.3 & 67 & 29.7 & 30 & $"$ \\
\hline 50 & & 40 & 12 & 67 & 04.2 & 30 & $"$ \\
\hline 51 & & 40 & 25.2 & 66 & 51.2 & 30 & $"$ \\
\hline 52 & & 40 & 43 & 66 & 46.8 & 30 & " \\
\hline 53 & & 41 & 02.1 & 66 & 43.4 & 30 & $"$ \\
\hline 54 & & 42 & 02.4 & 66 & 11.8 & 1 & May \\
\hline 55 & & 41 & 55.8 & 66 & 19.8 & 1 & $"$ \\
\hline 56 & & 41 & 37 & 66 & 32.7 & 1 & $"$ \\
\hline 57 & & 41 & 22.9 & 66 & 48.2 & 1 & " \\
\hline 58 & & 41 & 08.2 & 67 & 10.8 & 1 & $"$ \\
\hline 59 & & 40 & 56.8 & 67 & 31.4 & 1 & $"$ \\
\hline 60 & & 40 & 44.7 & 67 & 50.7 & 1 & $"$ \\
\hline
\end{tabular}

Recovered

Lat. Long. Date

No Returns

$\begin{array}{lllll}41 & 20.9 & 70 & 35 & 22 \text { Nov. } ' 57\end{array}$

$\begin{array}{lllll}40 & 35.4 & 73 & 34.6 & 3 \text { Sept.'57 }\end{array}$

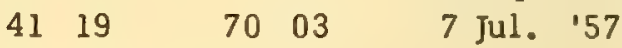

No Returns

$"$

"

$\begin{array}{llllll}41 & 27.7 & 71 & 10.2 & 22 & \text { Sept. } 57\end{array}$ 60

$$
00
$$

24 Nov. '58

No Returns

$\begin{array}{llllll}41 & 21 & 70 & 27 & 19 \mathrm{Jul} . & 157\end{array}$

$\begin{array}{lllll}41 & 36.5 & 69 & 59.3 & 11\end{array}$ Jan. '58 


\section{Albatross III Cruise 93}

$$
8 \text { - } 16 \text { May }
$$

\section{7}

12 bottles released at each station, odd numbers - ballasted, even numbers - no ballast.

Released

Sta.\# Bottle \# Lat. Long.

$1 \quad 31080 \quad 41 \quad 34.5 \quad 69 \quad 52$

31081

2

3

4

5

6

7

8

9

10

11

12

13

14

15

16

17

18

19
31082

31084

$41 \quad 34.5$

4134.5

4134.5

4134.5

4133

4132.3

4127.2

4124.1

31152

4122.2

4124.9

4133.8

31192

31194

31208

$41 \quad 32.5$

4132.5

4152

$42 \quad 10.9$

4150

31237

31241

31247

$41 \quad 49.2$

4149.2

4149.2

$41 \quad 37.9$

4130.6

4130.5

$41 \quad 28.7$

4154.4

31308

31312

31314

31315

31318
$42 \quad 10$

$42 \quad 10$

$42 \quad 10$

$42 \quad 10$

$42 \quad 10$
6952

6952

6952

$69 \quad 24$

$\begin{array}{ll}68 & 57.7\end{array}$

$\begin{array}{ll}68 & 31.8\end{array}$

$\begin{array}{ll}68 & 05.5\end{array}$

$\begin{array}{ll}67 & 41.8\end{array}$

$\begin{array}{ll}67 & 18.2\end{array}$

6654.1

$\begin{array}{ll}66 & 30.2\end{array}$

$\begin{array}{ll}66 & 30.2\end{array}$

$\begin{array}{ll}66 & 22.8\end{array}$

$\begin{array}{ll}66 & 10.5\end{array}$

$\begin{array}{ll}65 & 55.4\end{array}$

$\begin{array}{ll}65 & 26.9\end{array}$

$\begin{array}{ll}65 & 26.9\end{array}$

$\begin{array}{ll}65 & 26.9\end{array}$

$\begin{array}{ll}65 & 16\end{array}$

$65 \quad 25$

$\begin{array}{ll}65 & 48\end{array}$

$\begin{array}{ll}66 & 08.5\end{array}$

$\begin{array}{ll}65 & 40.3\end{array}$

$\begin{array}{ll}65 & 36.5\end{array}$

6536.5

6536.5

6536.5

$65 \quad 36.5$
Date

8 May

8 "

8 "

8 ."

8 "

8 "

8 "

8 "

8 "

9 "

9 "

9 "

9 ."

9 "

9 "

9 "

$9 "$

9 "

$9 "$

9 "

10 "

$10 "$

$10 "$

$10 \quad 1$

$10 "$

$10 "$

$10 "$

10 "

$10 \quad 11$

$10 "$
Recovered

Lat. Long. Late

$\begin{array}{llllll}41 & 32.5 & 70 & 36.4 & 12 \text { Jun. '57 }\end{array}$

$\begin{array}{llllll}41 & 42 & 70 & 39.8 & 17 & \text { Aug. '57 }\end{array}$

$\begin{array}{llllll}41 & 30 & 71 & 03 & 15 & \text { Jun. ' } 57\end{array}$

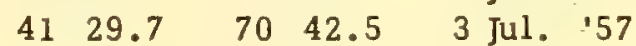

No Returns

" "

"

"

$\begin{array}{lllll}52 & 31 & 09 & 10 & 28 \text { Sept。'58 }\end{array}$

No Returns

$\begin{array}{lllllll}41 & 39.1 & 70 & 47 & 20 & \text { Aug. '57 }\end{array}$

$\begin{array}{llllll}41 & 14.6 & 70 & 05 & 12 \mathrm{Jul} . & 157\end{array}$

$\begin{array}{lllll}43 & 58 & 58 & 47 & 16 \text { Sept.'57 }\end{array}$

No Returns

$\begin{array}{llllll}44 & 23.8 & 66 & 12.2 & 12 & \text { Aug • } 57\end{array}$

$\begin{array}{lllll}44 & 40 & 66 & 45 & 20 \text { Nov. '57 }\end{array}$

$\begin{array}{lllll}44 & 43.6 & 62 & 56 & 27 \\ \text { Jun. '59 }\end{array}$

No Returns

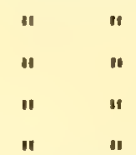

$\begin{array}{llllll}45 & 01 & 66 & 55.7 & 31 \text { Aug. } 157\end{array}$

$\begin{array}{llllll}58 & 35 & 03 & 25 & 6 & \text { Aug. '59 }\end{array}$

$\begin{array}{llllll}45 & 00.1 & 66 & 53.8 & 3 \mathrm{Jul} . & \text { "57 }\end{array}$



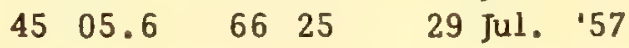


Released

\begin{tabular}{|c|c|c|c|c|c|c|c|}
\hline Sta. \# & Bottle \# & & at. & & ng. & & ate \\
\hline 20 & & 42 & 09.3 & 66 & 02 & 10 & May \\
\hline 21 & 31342 & 42 & 08.5 & 66 & 21.5 & 10 & $"$ \\
\hline 22 & & 42 & 06 & 66 & 27.5 & 11 & $"$ \\
\hline 23 & & 42 & 05.5 & 66 & 49 & 11 & " \\
\hline 24 & 31374 & 42 & 06.7 & 67 & 13.5 & 11 & $"$ \\
\hline 25 & 31384 & 42 & 03.6 & 67 & 32 & 11 & $"$ \\
\hline 26 & & 41 & 50 & 67 & 21 & 11 & $"$ \\
\hline 27 & & 41 & 48.2 & 66 & 51.8 & 11 & $"$ \\
\hline 28 & & 41 & 47.2 & 66 & 36.8 & 11 & $"$ \\
\hline 29 & & 41 & 48.8 & 66 & 13.8 & 11 & $"$ \\
\hline 30 & & 41 & 29.3 & 66 & 25.4 & 11 & $"$ \\
\hline 31 & & 41 & 15.8 & 66 & 30.8 & 11 & $"$ \\
\hline 32 & & 41 & 15.7 & 66 & 04.3 & 11 & $"$ \\
\hline 33 & & 41 & 00 & 66 & 19.5 & 12 & $"$ \\
\hline 34 & 31492 & 40 & 57.9 & 66 & 47.5 & 12 & $"$ \\
\hline 35 & 31510 & 40 & 58.8 & 67 & 15 & 12 & $"$ \\
\hline 36 & & 41 & 00.8 & 67 & 42.1 & 12 & $"$ \\
\hline 37 & 31524 & 41 & 01 & 68 & 00 & 12 & $"$ \\
\hline & 31526 & 41 & 01 & 68 & 00 & 12 & $"$ \\
\hline & 31528 & 41 & 01 & 68 & 00 & 12 & $"$ \\
\hline & 31530 & 41 & 01 & 68 & 00 & 12 & $"$ \\
\hline 38 & & 41 & 00 & 68 & 23.2 & 12 & $"$ \\
\hline 39 & 31548 & 40 & 42 & 68 & 28.8 & 12 & $"$ \\
\hline & 31549 & 40 & 42 & 68 & 28.8 & 12 & $"$ \\
\hline & 31552 & 40 & 42 & 68 & 28.8 & 12 & " \\
\hline & 31555 & 40 & 42 & 68 & 28.8 & 12 & $"$ \\
\hline & 31556 & 40 & 42 & 68 & 28.8 & 12 & $"$ \\
\hline & 31558 & 40 & 42 & 68 & 28.8 & 12 & " \\
\hline 40 & 31568 & 40 & 38.5 & 68 & 06.8 & 12 & $"$ \\
\hline 41 & 31572 & 40 & 39 & 67 & 42.9 & 12 & $"$ \\
\hline 42 & 31586 & 40 & 38 & 67 & 20 & 12 & $"$ \\
\hline 43 & & 40 & 40.4 & 66 & 45 & 12 & $"$ \\
\hline 44 & & 40 & 31.6 & 66 & 38.5 & 13 & $"$ \\
\hline 45 & & 40 & 20.5 & 66 & 56.8 & 13 & " \\
\hline 46 & & 40 & 19.5 & 67 & 19.5 & 13 & $"$ \\
\hline 47 & & 40 & 17.7 & 67 & 46 & 13 & $"$ \\
\hline
\end{tabular}

Recovered

Lat. Long 。 Date

\begin{tabular}{|c|c|c|}
\hline & No Returns & \\
\hline $45 \quad 22$ & $\begin{array}{c}6420.3 \\
\text { No Returns }\end{array}$ & 2 Sept.' 57 \\
\hline
\end{tabular}



$41 \quad 40 \quad 69 \quad 57 \quad 30$ Jun. '57 $\begin{array}{llllll}41 & 18.5 & 70 & 48 & 21 \text { Sept.'57 }\end{array}$ No Returns

$\begin{array}{llllrlr}41 & 15 & 70 & 10 & 14 \mathrm{Jul} . & 157 \\ 41 & 40.1 & 70 & 00 & 17 \mathrm{Dec} . & 57 \\ 41 & 20 & 70 & 40 & 6 \mathrm{Jul} . & ' 57 \\ 41 & 20.8 & 70 & 29 & 23 \mathrm{Jun} . & ' 57\end{array}$
$\begin{array}{lllll}41 & 39.5 & 69 & 59 & 26 \text { Jun. '57 }\end{array}$ $4130 \quad 71 \quad 02.5 \quad 3$ Aug. '57 $\begin{array}{llllll}41 & 23 & 70 & 30.3 & 24 \text { Jun. '57 }\end{array}$ $\begin{array}{lllll}41 & 28 & 70 & 34.6 & 9 \\ \text { JuI. } & 57\end{array}$ $\begin{array}{llllll}41 & 15.5 & 70 & 13.5 & 30 \text { Jun. '57 }\end{array}$ $41 \quad 32.9 \quad 70 \quad 31.9 \quad 30$ Jun. '57 $41 \quad 16.5 \quad 70 \quad 13.5 \quad 6$ Jul. 57 $\begin{array}{lllll}41 & 16.5 & 70 & 13.5 & 6 \text { Sept.'57 }\end{array}$ $\begin{array}{llllll}41 & 36.5 & 69 & 59 & 26 \mathrm{Jul} . & \text { '57 }\end{array}$ No Returns 


\section{Released}

\section{Sta.\# Bottle \# Lat. Long. Date}

$48 \quad 31659$

$49 \quad 31674$

50

51

52

53

54

55

56

57

58

59

60

61

62

63

64

65

66

67

68

69

\section{4}

31898

31909

31910

31914

31915

31916

31919

31921

31922

31925

31927

31929

31930

31931

31933

31934 $\begin{array}{llll}40 & 20.5 & 68 & 11\end{array}$

$\begin{array}{ll}40 & 20.3\end{array}$

$40 \quad 14$

$\begin{array}{ll}39 & 59.2\end{array}$

$40 \quad 00$

$40 \quad 04.5$

$\begin{array}{ll}40 & 22.7\end{array}$

$\begin{array}{ll}40 & 39.5\end{array}$

$40 \quad 55$

4108.2

4124.8

4108.2

$41 \quad 25$

4140.5

4131.6

$41 \quad 15.8$

4102

$40 \quad 56$

$40 \quad 54.5$

$40 \quad 52$

$40 \quad 47.5$

$40 \quad 40.6$

$40 \quad 40.6$

$40 \quad 40.6$

$40 \quad 40.6$

$40 \quad 40.6$

$40 \quad 40.6$

$40 \quad 37.4$

$40 \quad 37.4$

$40 \quad 37.4$

$40 \quad 37.4$

$40 \quad 37.4$

$\begin{array}{ll}40 & 37.4\end{array}$

$40 \quad 37.4$

$40 \quad 39.5$

$40 \quad 39.5$
$68 \quad 36$

$\begin{array}{llll}68 & 46.6 \quad 13 \quad "\end{array}$

$\begin{array}{lll}68 & 43.5 \quad 13 \text { " }\end{array}$

$\begin{array}{llll}68 & 21 & 13\end{array}$

$\begin{array}{llll}67 & 57.5 & 13\end{array}$

$\begin{array}{llll}67 & 45.8 \quad 13 \quad\end{array}$

$\begin{array}{llll}67 & 33.4 \quad 13 \quad "\end{array}$

$\begin{array}{llll}67 & 18.4 \quad 14\end{array}$

$\begin{array}{llll}67 & 03 & 14\end{array}$

$\begin{array}{llll}66 & 55.2 \quad 14\end{array}$

$\begin{array}{llll}65 & 23.8 \quad 14\end{array}$

$\begin{array}{llll}65 & 38.5 \quad 14 \quad\end{array}$

$\begin{array}{lll}65 & 56.8 \quad 15 \quad "\end{array}$

$\begin{array}{llll}66 & 05.8 \quad 15 \quad "\end{array}$

$\begin{array}{llll}66 & 23.1 & 15 \quad "\end{array}$

$\begin{array}{llll}66 & 44 & 15\end{array}$

$\begin{array}{llll}66 & 54.8 \quad 15 \quad "\end{array}$

$\begin{array}{lll}67 & 28.7 & 15\end{array}$

$\begin{array}{lll}67 & 49.7 & 15\end{array}$

$\begin{array}{ll}68 & 06\end{array}$

6826

$\begin{array}{ll}68 & 26\end{array}$

$68 \quad 26$

$68 \quad 26$

$68 \quad 26$

$\begin{array}{ll}68 & 26\end{array}$

$\begin{array}{ll}68 & 51.5\end{array}$

$68 \quad 51.5$

$\begin{array}{ll}68 & 51.5\end{array}$

$\begin{array}{ll}68 & 51.5\end{array}$

$68 \quad 51.5$

$68 \quad 51.5$

$68 \quad 51.5$

$69 \quad 15.5$

$69 \quad 15.5$
Recovered

Lat.

Long.

Date

$\begin{array}{llllll}35 & 38 & 75 & 27.5 & 1\end{array}$

$\begin{array}{llllll}41 & 39.2 & 70 & 07.5 & 30 & \text { Jun. '57 }\end{array}$

No Returns

$\begin{array}{lllll}40 & 38 & 73 & 12 & 13\end{array}$ Sept.'57

No Returns

" "

" 1

"

$\begin{array}{llllll}41 & 39.3 & 70 & 07.5 & \text { I Aug. '57 }\end{array}$

No Returns

$\begin{array}{lllll}40 & 38.6 & 73 & 09.3 & 16 \text { Sept. } 57\end{array}$

No Returns

$\begin{array}{llllll}45 & 18 & 64 & 24 & 9 \text { Nov. } 157\end{array}$

$\begin{array}{lllll}41 & 21.8 & 70 & 29 & 30 \text { Jun. '57 }\end{array}$

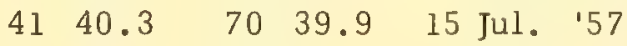

$\begin{array}{lllllll}41 & 37 & 70 & 16 & 28 & \text { Jul. } & 157\end{array}$

$\begin{array}{llllll}40 & 35 & 73 & 39 & 19 & \text { Sept.' } 57\end{array}$

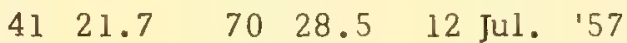

$\begin{array}{lllll}41 & 21 & 70 & 29 & 24 \text { Jun. '57 }\end{array}$

$\begin{array}{lllll}41 & 20.9 & 70 & 34 & 25 \text { Jun. '57 }\end{array}$

$\begin{array}{lllll}41 & 39.2 & 70 & 07.5 & 29 \\ \text { Jun. ' } 57\end{array}$

$\begin{array}{llllll}41 & 39.8 & 70 & 05.3 & 28 \\ \text { Jun. '57 }\end{array}$

$\begin{array}{llllll}41 & 39.5 & 69 & 59 & 8 \mathrm{Jul} . & 57\end{array}$

$\begin{array}{llllll}41 & 17.6 & 70 & 14.5 & 3 \text { Jul. } & 157\end{array}$

$\begin{array}{llllll}41 & 20.5 & 70 & 42.5 & 30 \text { Jun. '57 }\end{array}$

$\begin{array}{lllll}41 & 21 & 70 & 28 & 24 \\ \text { Jun. '57 }\end{array}$

$\begin{array}{lllll}41 & 39.2 & 70 & 07.5 & 27 \\ \text { Jun. } & 57\end{array}$

$\begin{array}{llllll}41 & 20.8 & 70 & 30.6 & \text { Jul. } 57\end{array}$

$\begin{array}{llllll}41 & 30.5 & 71 & 04.5 & 30 & \text { Jun. '57 }\end{array}$ 
Released

\begin{tabular}{|c|c|c|c|c|c|c|c|}
\hline \multirow{2}{*}{$\begin{array}{l}\text { Sta.\# } \\
71\end{array}$} & \multirow{2}{*}{$\begin{array}{c}\text { Bottle \# } \\
31937\end{array}$} & \multicolumn{2}{|c|}{ Lat. } & \multicolumn{2}{|c|}{ Long. } & \multicolumn{2}{|c|}{ Date } \\
\hline & & 40 & 39.5 & 69 & 15.5 & 15 & May \\
\hline & 31940 & 40 & 39.5 & 69 & 15.5 & 15 & $"$ \\
\hline & 31941 & 40 & 39.5 & 69 & 15.5 & 15 & $"$ \\
\hline & 31942 & 40 & 39.5 & 69 & 15.5 & 15 & " \\
\hline & 31943 & 40 & 39.5 & 69 & 15.5 & 15 & $"$ \\
\hline \multirow[t]{4}{*}{72} & 31944 & 40 & 37.4 & 69 & 40 & 15 & $"$ \\
\hline & 31950 & 40 & 37.4 & 69 & 40 & 15 & $"$ \\
\hline & 31954 & 40 & 37.4 & 69 & 40 & 15 & $"$ \\
\hline & 31955 & 40 & 37.4 & 69 & 40 & 15 & $"$ \\
\hline \multirow[t]{5}{*}{73} & 31956 & 40 & 41 & 70 & 03 & 15 & $"$ \\
\hline & 31957 & 40 & 41 & 70 & 03 & 15 & $"$ \\
\hline & 31959 & 40 & 41 & 70 & 03 & 15 & $"$ \\
\hline & 31966 & 40 & 41 & 70 & 03 & 15 & $"$ \\
\hline & 31967 & 40 & 41 & 70 & 03 & 15 & $"$ \\
\hline \multirow[t]{3}{*}{74} & 31972 & 40 & 51.7 & 70 & 20 & 16 & $"$ \\
\hline & 31974 & 40 & 51.7 & 70 & 20 & 16 & $"$ \\
\hline & 31975 & 40 & 51.7 & 70 & 20 & 16 & $"$ \\
\hline
\end{tabular}

Recovered

Long 。 Date

$\begin{array}{lllllll}41 & 20.8 & 70 & 30.6 & \text { Jul. '57 } \\ 41 & 19 & 70 & 48.5 & 18 \text { Jun. '57 } \\ 41 & 39.2 & 70 & 07.5 & 25 \text { Jun. '57 } \\ 41 & 20 & 70 & 49.5 & 25 \text { Jun. '57 } \\ 41 & 23 & 70 & 30.3 & 24 \text { Jun. '57 } \\ 41 & 38.4 & 70 & 13 & 25 \text { Oct. '57 } \\ 41 & 20.9 & 70 & 35 & 22 \text { Nov. '57 } \\ 40 & 52.5 & 72 & 22.5 & 12 \text { Sept.' } 57 \\ 41 & 20.8 & 70 & 30.6 & \text { Jul. '57 } \\ 39 & 57.4 & 74 & 04 & \text { 23 Aug. '57 } \\ 41 & 38.6 & 70 & 15.3 & 10 \text { Jul. '57 } \\ 41 & 39.4 & 70 & 06.9 & 10 \text { Jul. '57 } \\ 41 & 35.3 & 70 & 50 & 1 \text { Jul. '57 } \\ 41 & 38.5 & 70 & 15 & 7 \text { Jul. '57 } \\ 41 & 33 & 70 & 34.5 & \text { 28 Jul. '57 } \\ 41 & 30.5 & 71 & 04 & 1 \text { Aug. '57 } \\ 41 & 23.5 & 70 & 03 & 19 \text { May '60 }\end{array}$




\section{$\underline{\text { Albatross III Cruise } 94}$}

$$
22-28 \text { May }
$$

1957

12 bottles released at each station, odd numbers - ballasted, even numbers - no ballast.

Released

\begin{tabular}{|c|c|c|c|c|c|c|c|}
\hline Sta.\# & Bottle \# & & at. & & ing. & & ate \\
\hline 1 & 31980 & 41 & 26.5 & 69 & 43.5 & 22 & May \\
\hline 2 & & 41 & 22 & 69 & 24 & 22 & $"$ \\
\hline 3 & & 41 & 11 & 69 & 05 & 22 & $"$ \\
\hline 4 & & 41 & 02.5 & 68 & 40 & 22 & $"$ \\
\hline 5 & & 41 & 00.8 & 68 & 14.8 & 22 & $"$ \\
\hline 6 & & 41 & 01 & 67 & 47.8 & 23 & $"$ \\
\hline 7 & 32056 & 40 & 53 & 67 & 48.5 & 23 & $"$ \\
\hline 8 & & 40 & 42.2 & 67 & 49 & 23 & $"$ \\
\hline 9 & 32084 & 40 & 44 & 67 & 24 & 23 & $"$ \\
\hline 10 & & 40 & 45.2 & 67 & 01.1 & 23 & $"$ \\
\hline 11 & & 40 & 46.5 & 66 & 55.5 & 23 & $"$ \\
\hline 12 & & 41 & 03.2 & 67 & 02.2 & 23 & $"$ \\
\hline 13 & & 41 & 16.3 & 67 & 09 & 23 & $"$ \\
\hline 14 & 32138 & 41 & 37 & 67 & 18.5 & 23 & $"$ \\
\hline 15 & & 41 & 48.4 & 67 & 19.2 & 23 & $"$ \\
\hline 16 & & 41 & 50.2 & 66 & 51.5 & 23 & $"$ \\
\hline 17 & 32181 & 41 & 49.7 & 66 & 23.2 & 23 & $"$ \\
\hline 18 & & 41 & 49.9 & 65 & 55.8 & 23 & $"$ \\
\hline 19 & 32198 & 41 & 49.3 & 65 & 35.6 & 24 & $"$ \\
\hline & 32207 & 41 & 49.3 & 65 & 35.6 & 24 & $"$ \\
\hline 20 & & 41 & 38.6 & 65 & 25.3 & 24 & $"$ \\
\hline 21 & & 41 & 25.8 & 65 & 46.5 & 24 & $"$ \\
\hline 22 & & 41 & 25.8 & 65 & 46.5 & 24 & " \\
\hline 23 & & 41 & 25.8 & 66 & 05 & 24 & $"$ \\
\hline 24 & & 41 & 24 & 66 & 23.3 & 24 & $"$ \\
\hline 25 & & 41 & 25 & 66 & 43 & 24 & $"$ \\
\hline 26 & & 41 & 27 & 67 & 10 & 24 & " \\
\hline 27 & & 41 & 29.3 & 67 & 34.8 & 24 & " \\
\hline 28 & 32308 & 41 & 16 & 67 & 36.2 & 24 & " \\
\hline
\end{tabular}

Recovered

Lat. Long. Date

$\begin{array}{lllll}44 & 34 & 65 & 55 & 16\end{array}$

No Returns

" "

" 1

" 1

"

$\begin{array}{llllll}41 & 19 & 70 & 48.5 & 24 & \text { Aug. '57 }\end{array}$ No Returns

$\begin{array}{llllll}40 & 37.3 & 73 & 17 & 14 & \text { Sept.'57 }\end{array}$

No Returns

" "

" 1

"

$\begin{array}{llllll}57 & 27 & 07 & 30 & 8 \text { Nov . '58 }\end{array}$

No Returns

" "

Norway 31 Dec. '58

No Returns

$\begin{array}{llllll}44 & 34 & 65 & 54.5 & 1\end{array}$ Aug. '57

$\begin{array}{lllll}45 & 19 & 64 & 25 & 15\end{array}$ Sept.'57

No Returns

" "

"

"

" "

" 1

" "

" 1

$\begin{array}{llllll}41 & 20 & 70 & 49.5 & 7 & \text { Oct. }\end{array}$ 
Released

\begin{tabular}{|c|c|c|c|c|c|c|c|}
\hline Sta.\# & Bottle \# & & at. & Lo & ng. & & ate \\
\hline 29 & & 41 & 11.7 & 67 & 14.2 & 24 & May \\
\hline 30 & & 41 & 10.3 & 66 & 45.2 & 24 & $"$ \\
\hline 31 & & 41 & 09.7 & 66 & 29.3 & 25 & $"$ \\
\hline 32 & & 41 & 08.5 & 66 & 01.6 & 25 & " \\
\hline 33 & & 40 & 45 & 65 & 20 & 25 & " \\
\hline 34 & & 41 & 04.1 & 66 & 14.3 & 25 & $"$ \\
\hline 35 & & 40 & 49.7 & 66 & 20.1 & 25 & $"$ \\
\hline 36 & & 40 & 49.8 & 66 & 39.1 & 25 & $"$ \\
\hline 37 & 32416 & 40 & 39 & 67 & 17 & 25 & $"$ \\
\hline 38 & & 40 & 34.2 & 67 & 40.8 & 26 & $"$ \\
\hline 39 & 32438 & 40 & 34.9 & 67 & 53 & 26 & $"$ \\
\hline 40 & & 40 & 36.5 & 68 & 23.1 & 26 & $"$ \\
\hline 41 & 32464 & 40 & 45.9 & 68 & 40.8 & 26 & $"$ \\
\hline 42 & & 40 & 50.7 & 68 & 16.4 & 26 & $"$ \\
\hline 43 & & 40 & 50.4 & 67 & 49 & 26 & " \\
\hline 44 & & 40 & 51.5 & 67 & 19.5 & 26 & $"$ \\
\hline 45 & 32513 & 40 & 48.4 & 67 & 09.4 & 26 & $"$ \\
\hline 46 & & 40 & 44 & 66 & 53 & 26 & " \\
\hline 47 & & 40 & 36.2 & 66 & 33 & 26 & " \\
\hline 48 & & 40 & 28.5 & 66 & 05.8 & 26 & " \\
\hline 49 & & 40 & 20 & 66 & 09.1 & 26 & " \\
\hline 50 & & 40 & 20.2 & 66 & 22.6 & 26 & " \\
\hline 51 & & 40 & 22 & 66 & 37.5 & 27 & " \\
\hline 52 & & 40 & 23.5 & 66 & 56 & 27 & $"$ \\
\hline 53 & 32604 & 40 & 24.5 & 67 & 15.5 & 27 & $"$ \\
\hline 54 & 32620 & 40 & 27 & 67 & 34.5 & 27 & " \\
\hline 55 & 32628 & 40 & 20.9 & 67 & 57.2 & 27 & $"$ \\
\hline 56 & & 40 & 20.5 & 68 & 20.7 & 27 & $"$ \\
\hline 57 & 32662 & 40 & 17.8 & 68 & 36 & 27 & " \\
\hline 58 & 32666 & 40 & 18.7 & 69 & 01.3 & 27 & $"$ \\
\hline & 32668 & 40 & 18.7 & 69 & 01.3 & 27 & $"$ \\
\hline & 32669 & 40 & 18.7 & 69 & 01.3 & 27 & " \\
\hline & 32670 & 40 & 18.7 & 69 & 01.3 & 27 & $"$ \\
\hline & 32674 & 40 & 18.7 & 69 & 01.3 & 27 & $"$ \\
\hline & 32675 & 40 & 18.7 & 69 & 01.3 & 27 & $"$ \\
\hline
\end{tabular}

Recovered

Lat.

Long .

Date

No Returns

$41 \quad 17$

$\begin{array}{ll}70 & 13\end{array}$

No Returns

4121

$70 \quad 40$

No Returns

4356

$\begin{array}{ll}60 & 03\end{array}$

No Returns

" $"$

$41 \quad 30.5 \quad 71 \quad 05$

31 Jul. 157 No Returns

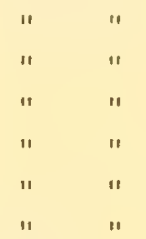

$\begin{array}{ll}51 & 08\end{array}$

$04 \quad 15$

$41 \quad 17.5$

$70 \quad 14.5$

17 Aug. '58

41 17

$\begin{array}{ll}70 & 14.5\end{array}$

No Returns

$32 \quad 17$

6444

25 Jun. 157

4140

$\begin{array}{ll}70 & 02.5\end{array}$

23 Nov. '57

4139.8

$\begin{array}{ll}70 & 04.5\end{array}$

28 Jun. '57

4123

$\begin{array}{ll}70 & 03\end{array}$

28 Jun. '57

4136.5

$\begin{array}{ll}69 & 59.5\end{array}$

16 Jul. 157

$41 \quad 14.6$

$\begin{array}{ll}70 & 07.7\end{array}$

23 Jan. 158

$\begin{array}{ll}41 & 29.8\end{array}$ $\begin{array}{ll}71 & 02.5\end{array}$
25 Jun. 57

12 Aug. '57 
Albatross III Cruise 94 (cont'd)

Released

\begin{tabular}{|c|c|c|c|c|c|c|c|}
\hline Sta.\# & Bottle \# & & at. & & ng . & & ate \\
\hline 59 & & 40 & 00 & 69 & 01 & 27 & May \\
\hline 60 & 32697 & 40 & 00 & 68 & 35.3 & 27 & $"$ \\
\hline 61 & & 40 & 02.8 & 68 & 10.8 & 27 & $"$ \\
\hline 62 & & 40 & 16 & 67 & 57.7 & 27 & $"$ \\
\hline 63 & 32726 & 40 & 23 & 67 & 47 & 28 & $"$ \\
\hline 64 & & 40 & 31.3 & 67 & 34.9 & 28 & $"$ \\
\hline 65 & 32748 & 40 & 36 & 67 & 35 & 28 & $"$ \\
\hline & 32750 & 40 & 36 & 67 & 35 & 28 & $"$ \\
\hline & 32758 & 40 & 36 & 67 & 35 & 28 & $"$ \\
\hline 66 & & 40 & 55.3 & 67 & 32.4 & 28 & $"$ \\
\hline 67 & & 41 & 16 & 67 & 28 & 28 & $"$ \\
\hline 68 & & 41 & 35.6 & 66 & 54 & 28 & $"$ \\
\hline 69 & & 41 & 33.5 & 67 & 06 & 28 & $"$ \\
\hline 70 & & 41 & 30 & 67 & 30 & 28 & $"$ \\
\hline 71 & 32828 & 41 & 25.2 & 68 & 19 & 28 & $"$ \\
\hline
\end{tabular}

Recovered

Lat. Long. Date

No Returns

$6415 \quad 10 \quad 19 \mathrm{E} \quad 21$ Jun. '59
No Returns

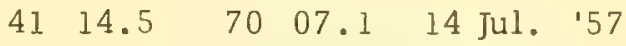
No Returns

$\begin{array}{lllllll}41 & 15.5 & 70 & 11.2 & 7 & \text { Jul. } & 57\end{array}$

$\begin{array}{lllll}40 & 50.5 & 72 & 29 & 24 \\ \text { Sept.'57 }\end{array}$

$41 \quad 20.5 \quad 70 \quad 42.5 \quad 21$ Sept.'57 No Returns

$\begin{array}{lrl}" & " & \\ " & " & \\ " & " & \\ 65 & 51 & 28 \text { Sept. } 57\end{array}$




\section{Albatross III Cruise 95}

$$
5 \text { - } 9 \text { June }
$$

1957

12 bottles released at each station, odd numbers - ballasted, even numbers - no ballast.

\section{Released}

\begin{tabular}{|c|c|c|c|c|c|c|c|}
\hline Sta.\# & Bottle \# & \multicolumn{2}{|c|}{ Lat. } & \multicolumn{2}{|c|}{ Long. } & \multicolumn{2}{|c|}{ Date } \\
\hline 1 & & 41 & 29 & 69 & 31 & 5 & Jun. \\
\hline 2 & 32862 & 41 & 34.5 & 69 & 09 & 5 & $"$ \\
\hline 3 & 32870 & 41 & 39 & 68 & 46.8 & 5 & $"$ \\
\hline 4 & 32886 & 41 & 44.8 & 68 & 20 & 5 & $"$ \\
\hline 5 & & 41 & 51 & 67 & 55 & 5 & $"$ \\
\hline 6 & & 42 & 00.5 & 67 & 34.2 & 6 & $n$ \\
\hline 7 & & 42 & 07.7 & 67 & 10.4 & 6 & $"$ \\
\hline 8 & 32934 & 42 & 06.2 & 66 & 48.3 & 6 & $n$ \\
\hline 9 & & 41 & 48.1 & 66 & 49 & 6 & $"$ \\
\hline 10 & & 41 & 28.4 & 66 & 52.1 & 6 & $n$ \\
\hline 11 & & 41 & 33.3 & 66 & 37.1 & 6 & $"$ \\
\hline 12 & & 41 & 39.4 & 66 & 09.2 & 6 & $"$ \\
\hline 13 & & 41 & 37 & 65 & 47 & 6 & $"$ \\
\hline 14 & & 41 & 21.9 & 65 & 54.6 & 6 & $"$ \\
\hline 15 & & 41 & 14.8 & 66 & 12.1 & 6 & " \\
\hline 16 & & 41 & 13.5 & 66 & 37.7 & 6 & $"$ \\
\hline 17 & & 41 & 13.8 & 66 & 58.5 & 7 & $"$ \\
\hline 18 & & 41 & 16.7 & 67 & 26.8 & 7 & $"$ \\
\hline 19 & & 41 & 18.8 & 67 & 51.5 & 7 & $"$ \\
\hline 20 & & 41 & 15.8 & 68 & 13.8 & 7 & $"$ \\
\hline 21 & & $4 I$ & 13 & 68 & 34.9 & 7 & $"$ \\
\hline 22 & & 40 & 52.8 & 68 & 29.8 & 7 & $"$ \\
\hline 23 & & 40 & 53.8 & 68 & 12.8 & 7 & $"$ \\
\hline 24 & & 40 & 56.7 & 67 & 49.5 & 7 & w \\
\hline 25 & & 40 & 57.9 & 67 & 25 & 7 & " \\
\hline 26 & & 40 & 57 & 66 & 57.2 & 7 & $"$ \\
\hline 27 & & 40 & 57.3 & 66 & 38 & 7 & $"$ \\
\hline 28 & & 40 & 48.8 & 66 & 21.8 & 8 & $"$ \\
\hline 29 & & 40 & 41.8 & 66 & 26.1 & 8 & $"$ \\
\hline 30 & & 40 & 37.5 & 66 & 52 & 8 & " \\
\hline
\end{tabular}

\section{Recovered}

Lat. Long. Date

No Returns

\begin{tabular}{rrrrrr}
44 & 40 & 67 & 20 & 15 Oct. '57 \\
43 & 30 & 65 & 35.5 & 19 Aug. '58 \\
43 & 58 & 58 & 47 & 9 Sept. '57 \\
& \multicolumn{4}{c}{ No Returns } \\
& \multicolumn{4}{c}{ " " } \\
& \multicolumn{4}{c}{ " } &
\end{tabular}

$43 \quad 56$ 


\section{Albatross III Cruise 95 (cont'd)}

\section{Released}

Sta.\#

31

32

33

34

35

36

37

38

39

40

41

42

43

44

45

46
Bottle \#



Long .

Date

$\begin{array}{llll}67 & 17.5 & 8 & \text { Jun. }\end{array}$

$40 \quad 40$

$40 \quad 41.6$

$\begin{array}{ll}40 & 39.9\end{array}$

$\begin{array}{lll}67 & 42.7\end{array}$

$\begin{array}{ll}68 & 08.6\end{array}$

$\begin{array}{ll}68 & 33.7\end{array}$

$\begin{array}{ll}68 & 52.9\end{array}$

6858

6858

$\begin{array}{ll}68 & 15\end{array}$

$\begin{array}{ll}68 & 10\end{array}$

$\begin{array}{ll}67 & 44.7\end{array}$

$\begin{array}{ll}67 & 10.8\end{array}$

6720

$40 \quad 12.8$

$40 \quad 11.3$

$40 \quad 11.2$

$\begin{array}{ll}40 & 10.7\end{array}$

$\begin{array}{ll}40 & 09.6\end{array}$

$\begin{array}{ll}67 & 41\end{array}$

6751

6826.3

$\begin{array}{ll}68 & 50.5\end{array}$

6915.5
Recovered

Lat. Long. Date

No Returns

" "

$\begin{array}{llllll}41 & 20.5 & 70 & 42.5 & 21 & \text { Sept. } 157\end{array}$

$\begin{array}{llllll}41 & 39.3 & 70 & 07 & 17 \text { Sept.'57 }\end{array}$

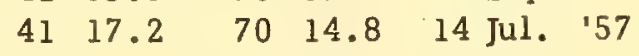

No Returns

" "

It

11

11

II II

II

II

II II

$\begin{array}{llllll}40 & 36.8 & 73 & 24.4 & 22 & \text { Sept.'57 }\end{array}$ 
9 - 26 September

1957

At stations where 12 bottles were released, six were ballasted, six had no ballast.

At stations where 6 bottles were released, all were ballasted.

Released

Recuvered

No.

Bottles

Sta.\# Released Bottle \# Lat. Long. Date Lat. Long. Date

\begin{tabular}{|c|c|c|c|c|c|c|c|c|c|c|c|c|c|}
\hline 95 & 12 & $\begin{array}{l}37575 \mathrm{~N} \\
37576 \mathrm{~N}\end{array}$ & $\begin{array}{l}44 \\
44\end{array}$ & $\begin{array}{l}57.8 \\
57.8\end{array}$ & $\begin{array}{l}66 \\
66\end{array}$ & $\begin{array}{l}51.2 \\
51.2\end{array}$ & $\begin{array}{l}9 \\
9\end{array}$ & $\begin{array}{l}\text { Sept. } \\
\text { " }\end{array}$ & $\begin{array}{l}44 \\
44\end{array}$ & $\begin{array}{l}55.9 \\
57.4\end{array}$ & $\begin{array}{l}66 \\
66\end{array}$ & $\begin{array}{l}56.4 \\
54.3\end{array}$ & $\begin{array}{l}17 \text { Sept. } ' 57 \\
20 \text { Nov. } 57\end{array}$ \\
\hline 94 & 12 & & 44 & 30.0 & 67 & 13.0 & 10 & $"$ & \multicolumn{5}{|c|}{ No Returns } \\
\hline 92 & 12 & & 44 & 00.0 & 67 & 38.8 & 10 & $"$ & & & $"$ & $"$ & \\
\hline 91 & 12 & & 44 & 00.0 & 68 & 34.0 & 10 & $"$ & & & $"$ & $"$ & \\
\hline 90 & 12 & & 43 & 41.0 & 68 & 51.0 & 10 & $"$ & & & $"$ & $"$ & \\
\hline 89 & 12 & $37036 \mathrm{~B}$ & 43 & 41.0 & 69 & 01.0 & 10 & $"$ & 43 & 40 & 65 & 09 & 12 Dec. '57 \\
\hline 88 & 12 & $37611 \mathrm{~N}$ & 43 & 22.2 & 69 & 01.0 & 10 & $"$ & 44 & 06 & 66 & 13 & 21 Oct. '57 \\
\hline & & $37607 \mathrm{~N}$ & 43 & 22.2 & 69 & 01.0 & 10 & $"$ & 43 & 41 & 66 & 01 & 29 Oct. '57 \\
\hline 87 & 12 & & 43 & 22.0 & 70 & 21.5 & 11 & $"$ & \multicolumn{5}{|c|}{ No Returns } \\
\hline 86 & 12 & & 42 & 35.2 & 69 & 29.5 & 11 & $"$ & & & $"$ & $"$ & \\
\hline 85 & 12 & & 41 & 51.0 & 70 & 26.0 & 11 & $"$ & & & $"$ & $"$ & \\
\hline 84 & 12 & & 42 & 20.0 & 70 & 38.8 & 11 & $"$ & & & $"$ & $"$ & \\
\hline 82 & 12 & & 42 & 31.2 & 70 & 18.2 & 11 & $"$ & & & $"$ & $"$ & \\
\hline 83 & 12 & & 42 & 41.8 & 70 & 00.0 & 12 & $"$ & & & $"$ & $"$ & \\
\hline 81 & 12 & & 42 & 57.5 & 70 & 00.0 & 12 & $"$ & & & $"$ & $"$ & \\
\hline 80 & 12 & $37687 \mathrm{~B}$ & 42 & 55.8 & 69 & 41.5 & 12 & $"$ & 43 & 42.1 & 66 & 03.5 & 27 Dec. '57 \\
\hline 79 & 12 & & 42 & 51.2 & 68 & 54.8 & 12 & $"$ & \multicolumn{5}{|c|}{ No Returns } \\
\hline 78 & 12 & & 42 & 46.5 & 68 & 00.0 & 12 & $"$ & & & $"$ & $"$ & \\
\hline 77 & 12 & & 42 & 34.5 & 65 & 54.0 & 13 & $"$ & & & $"$ & $"$ & \\
\hline 76 & 12 & & 42 & 30.8 & 65 & 10.5 & 13 & $"$ & & & $"$ & " & \\
\hline 75 & 12 & $37689 \mathrm{~B}$ & 43 & 22.1 & 66 & 02.1 & 13 & $"$ & 44 & 20 & 66 & 07.6 & 2 Dec. ' 57 \\
\hline & & $37118 \mathrm{~B}$ & 43 & 22.1 & 66 & 02.1 & 13 & $"$ & 44 & 54.3 & 65 & 21.2 & 13 Nov. 57 \\
\hline 96 & 12 & & 43 & 40.5 & 66 & 11.0 & 18 & $"$ & \multicolumn{5}{|c|}{ No Returns } \\
\hline 97 & 12 & $37127 \mathrm{~B}$ & 43 & 24.0 & 66 & 30.0 & 18 & " & 44 & 20 & 66 & 07.5 & 5 Oct. '57 \\
\hline & & $37132 \mathrm{~B}$ & 43 & 24.0 & 66 & 30.0 & 18 & $"$ & 44 & 13.6 & 66 & 08.5 & 13 Jun. '59 \\
\hline 98 & 12 & & 43 & 08.0 & 66 & 44.5 & 18 & $"$ & \multirow{2}{*}{\multicolumn{5}{|c|}{$\begin{array}{l}\text { No Returns } \\
\text { " }\end{array}$}} \\
\hline 99 & 12 & & 42 & 51.0 & 66 & 59.0 & 18 & $"$ & & & & & \\
\hline
\end{tabular}


Harengus Cruise 17 (cont'd)

Released

Recovered

No.

Bottles

Sta.\# Released Bottle \# Lat. Long. Date Lat. Long. Date

\begin{tabular}{|c|c|c|c|c|c|c|c|c|c|c|c|c|}
\hline 100 & 12 & & 42 & 34.0 & 67 & 13.5 & 18 & Sept. & & & No Returns & \\
\hline 101 & 12 & $37725 \mathrm{~N}$ & 42 & 21.0 & 67 & 21.0 & 18 & $"$ & 44 & 17 & 6620 & 20 Jan. \\
\hline 102 & 12 & $37729 \mathrm{~N}$ & 42 & I7. 0 & 67 & 27.8 & 18 & $"$ & 43 & 48 & 6453 & 8 Apr. \\
\hline 103 & 12 & & 42 & 09.0 & 67 & 34.5 & 18 & $"$ & & & No Returns & \\
\hline 104 & 12 & & 42 & 04.0 & 67 & 20.5 & 18 & $"$ & & & $"$ & \\
\hline 105 & 12 & & 42 & 12.0 & 67 & 20.2 & 19 & $"$ & & & $"$ & \\
\hline 106 & 12 & & 42 & 07.0 & 67 & 05.0 & 19 & $"$ & & & $"$ & \\
\hline 107 & 12 & & 42 & 15.5 & 67 & 52.5 & 19 & $"$ & 44 & 15.5 & $\begin{array}{ll}66 & 08\end{array}$ & 2 Dec. \\
\hline 08 & 12 & & 42 & 10.0 & 66 & 46.5 & 19 & $"$ & & & No Returns & \\
\hline 109 & 12 & & 42 & 06.0 & 66 & 34.0 & 19 & $"$ & & & $"$ & \\
\hline 110 & 12 & & 42 & 13.2 & 66 & 28.0 & 19 & $"$ & & & $"$ & \\
\hline 1 & 12 & & 42 & 23.0 & 66 & 26.2 & 19 & $"$ & & & $"$ & \\
\hline 112 & 12 & $37222 \mathrm{~B}$ & 42 & 32.5 & 66 & 24.5 & 19 & $"$ & 44 & 15 & $66 \quad 23$ & 23 Jan. \\
\hline 113 & 12 & $37226 \mathrm{~B}$ & 42 & 42.5 & 66 & 22.5 & 19 & $"$ & 44 & 03 & 6438 & 20 Nov. \\
\hline 114 & 12 & & 43 & 02.3 & 66 & 18.5 & 19 & $"$ & & & No Returns & \\
\hline 115 & 12 & $37806 \mathrm{~N}$ & 43 & 22.0 & 66 & 14.5 & 19 & $"$ & 43 & 52 & $\begin{array}{ll}66 & 10.5\end{array}$ & 29 Sept. \\
\hline 116 & 12 & & 43 & 40.5 & 66 & 10.8 & 19 & $"$ & & & No Returns & \\
\hline 36 & 12 & $37250 \mathrm{~B}$ & 43 & 40.5 & 66 & 10.8 & 24 & $"$ & 44 & 18 & $\begin{array}{ll}66 & 08\end{array}$ & 20 Nov. \\
\hline 35 & 12 & & 43 & 51.2 & 66 & 45.5 & 24 & $"$ & & & No Returns & \\
\hline 34 & 12 & & 44 & 06.2 & 66 & 21.0 & 24 & $"$ & & & $"$ & \\
\hline 33 & 12 & $37270 \mathrm{~B}$ & 44 & 18.0 & 66 & 43.0 & 24 & $"$ & 44 & 30 & $66 \quad 06$ & 27 Sept. \\
\hline 32 & 11 & $37843 \mathrm{~N}$ & 44 & 39.0 & 65 & 56.5 & 24 & $"$ & 44 & 41 & $\begin{array}{ll}65 & 48\end{array}$ & 28 Sept. \\
\hline 31 & 6 & & 44 & 51.5 & 66 & 03.5 & 24 & $"$ & & & No Returns & \\
\hline 30 & 5 & & 44 & 49.5 & 66 & 08.2 & 25 & $"$ & & & $"$ & \\
\hline 29 & 6 & & 44 & 52.5 & 66 & 12.8 & 25 & $"$ & & & $"$ & \\
\hline 28 & 6 & $37298 \mathrm{~B}$ & 44 & 55.5 & 66 & 17.8 & 25 & $"$ & 44 & 57 & $65 \quad 16$ & 13 Nov. \\
\hline 27 & 6 & & 44 & 58.8 & 66 & 22.5 & 25 & $"$ & & & No Returns & \\
\hline 26 & 12 & & 45 & 01.2 & 66 & 28.8 & 25 & $"$ & & & $"$ & \\
\hline Z & 6 & & 45 & 03.2 & 66 & 00.0 & 25 & $"$ & & & $"$ & \\
\hline $\mathrm{Y}$ & 6 & & 45 & 04.2 & 66 & 16.2 & 25 & $"$ & & & $"$ & \\
\hline 25 & 6 & & 45 & 10.2 & 65 & 56.8 & 25 & $"$ & & & $"$ & \\
\hline 24 & 6 & & 45 & 07.0 & 65 & 53.5 & 25 & $"$ & & & $"$ & \\
\hline 23 & 6 & & 45 & 03.8 & 65 & 49.7 & 25 & $"$ & & & $"$ & \\
\hline 22 & 12 & & 45 & 00.5 & 65 & 46.0 & 25 & $"$ & & & $"$ & \\
\hline
\end{tabular}


Harengus Cruise 17 (cont'd)

Released

Recovered

No.

Bottles

Sta.\# Released Bottle\# Lat. Long. Date Lat. Long. Date

\begin{tabular}{|c|c|c|c|c|c|c|c|c|c|c|c|c|c|}
\hline 21 & 6 & $37350 \mathrm{~B}$ & 44 & 57.5 & 65 & 42.5 & 25 & Sept. & 45 & 10.2 & $\begin{array}{ll}64 & 45.8\end{array}$ & 5 & Oct. \\
\hline 20 & 6 & $37359 \mathrm{~B}$ & 44 & 54.2 & 65 & 38.8 & 25 & $"$ & 44 & 57 & $65 \quad 16$ & 28 & Sept. \\
\hline 19 & 6 & & 44 & 51.0 & 65 & 35.0 & 25 & $"$ & & & No Returns & & \\
\hline 18 & 6 & $37370 \mathrm{~B}$ & 44 & 54.2 & 65 & 22.5 & 25 & $"$ & 45 & 13 & $\begin{array}{ll}64 & 31\end{array}$ & 20 & Oct. \\
\hline 17 & 6 & & 45 & 06.0 & 65 & 03.0 & 25 & $"$ & & & No Returns & & \\
\hline 16 & 6 & & 45 & 09.2 & 65 & 06.0 & 25 & $"$ & & & $"$ & & \\
\hline 15 & 6 & & 45 & 12.5 & 65 & 11.0 & 25 & $"$ & & & $"$ & & \\
\hline 14 & 12 & & 45 & 15.8 & 65 & 18.8 & 25 & $"$ & & & $"$ & & \\
\hline 13 & 6 & & 45 & 18.8 & 65 & 18.8 & 25 & $"$ & & & $"$ & & \\
\hline 12 & 6 & $37403 \mathrm{~B}$ & 45 & 22.2 & 65 & 22.8 & 25 & $"$ & 41 & 17.2 & $\begin{array}{ll}70 & 14.7\end{array}$ & 29 & Jun. \\
\hline il & 6 & & 44 & 43.8 & 66 & 00.0 & 25 & $"$ & & & No Returns & & \\
\hline 10 & 6 & & 44 & 18.8 & 66 & 21.2 & 25 & $"$ & & & $"$ & & \\
\hline 9 & 6 & $37421 \mathrm{~B}$ & 44 & 22.5 & 66 & 23.7 & 25 & $"$ & 44 & 30 & $66 \quad 06$ & 27 & Sept. \\
\hline 7 & 6 & & 44 & 31.5 & 66 & 29.8 & 26 & $"$ & & & No Returns & & \\
\hline 6 & 12 & 37437 B & 44 & 35.0 & 66 & 32.8 & 26 & $"$ & 44 & 16.8 & $\begin{array}{ll}66 & 08.2\end{array}$ & 5 & Oct. \\
\hline 5 & 6 & & 44 & 40.2 & 66 & 35.8 & 26 & $"$ & & & No Returns & & \\
\hline 4 & 6 & & 44 & 45.8 & 66 & 38.8 & 26 & $"$ & & & $"$ & & \\
\hline 3 & 6 & 37452 B & 44 & 49.0 & 66 & 42.0 & 26 & $"$ & 44 & 46.3 & $66 \quad 45.5$ & 8 & Oct. \\
\hline 2 & 6 & & 44 & 53.5 & 66 & 45.0 & 26 & $"$ & & & No Returns & & \\
\hline 1 & 12 & & 44 & 59.8 & 66 & 48.2 & 26 & $"$ & & & $"$ & & \\
\hline
\end{tabular}


Harengus Cruise 18

$7-25$ October

1957

At stations where 12 bottles were released, six were ballasted, six had no ballast.

At stations where 6 bottles were released, all were ballasted.

Released

Recovered

No.

Bottles

Sta.\# Released Bottle \# Lat. Long. Date Lat. Long. Date

95

12

$\begin{array}{lllllll}37878 \mathrm{~N} & 44 & 57.8 & 66 & 51.2 & 7 . & \text { Oct. } \\ 37879 & \mathrm{~N} & 44 & 57.8 & 66 & 51.2 & 7\end{array}$

$\begin{array}{llllll}44 & 57.3 & 67 & 02.4 & 9 & \text { Oct.' '57 }\end{array}$

$\begin{array}{llllllll}37880 \mathrm{~N} & 44 & 57.8 & 66 & 51.2 & 7 & \text { " }\end{array}$

\begin{tabular}{lllll}
44 & 52.2 & 67 & 00.1 & 12 \\
\hline & Oct. '57
\end{tabular}

$\begin{array}{lllll}45 & 03.8 & 66 & 50 & 21 \text { Nov. ' } 57\end{array}$

$\begin{array}{lllllll}37882 & N & 44 & 57.8 & 66 & 51.2 & 7\end{array}$

$\begin{array}{llllll}44 & 54 & 67 & 00.5 & 2 \text { Jan. '58 }\end{array}$

$\begin{array}{lllllll}37469 \text { B } & 44 & 57.8 & 66 & 51.2 & 7\end{array}$

$\begin{array}{llllll}44 & 53 & 66 & 57.1 & 11 & \text { Oct. ' } 57\end{array}$

$\begin{array}{lllllll}37470 & \text { B } & 44 & 57.8 & 66 & 51.2 & 7\end{array}$

$\begin{array}{lllll}44 & 57 & 66 & 54.7 & 3 \text { Nov. ' } 57\end{array}$

$\begin{array}{lllllllll}37472 & \text { B } & 44 & 57.8 & 66 & 51.2 & 7 & \text { " }\end{array}$

$\begin{array}{llllll}44 & 56.2 & 66 & 56 & 16 & \text { Oct. ' } 57\end{array}$

$\begin{array}{lllllllll}37473 & \text { B } & 44 & 57.8 & 66 & 51.2 & 7 & \text { " }\end{array}$

$\begin{array}{lllllll}44 & 50.4 & 66 & 56.4 & 11 & \text { Oct. '57 }\end{array}$

$\begin{array}{llllllll}37474 \text { B } & 44 & 57.8 & 66 & 51.2 & 7 & \text { " }\end{array}$

$\begin{array}{llllll}44 & 57.3 & 66 & 54.2 & 24 & \text { Oct. '57 }\end{array}$

$\begin{array}{llllll}44 & 31.2 & 67 & 12.0 & 7 & 11\end{array}$

No Returns

$\begin{array}{lllllll}43 & 50.8 & 66 & 09 & 22 & \text { Dec. } & 57\end{array}$

No Returns

$\begin{array}{lllllll}44 & 00.0 & 67 & 37.5 & 7 & 1 "\end{array}$

$\begin{array}{llllll}44 & 00.0 & 68 & 33.5 & 7 & \text { " }\end{array}$

$\begin{array}{lllllll}43 & 41.5 & 68 & 33.8 & 8 & \end{array}$

$\begin{array}{lllllll}43 & 41.5 & 69 & 00.0 & 8 & \end{array}$

$\begin{array}{llllll}43 & 22.0 & 70 & 21.0 & 8 & \end{array}$

$\begin{array}{llllll}43 & 01.5 & 69 & 50.2 & 8 & \end{array}$

$\begin{array}{llllll}43 & 00.0 & 69 & 00.0 & 8 & \end{array}$

$\begin{array}{llllll}43 & 22.5 & 69 & 00.0 & 8 & \end{array}$

$\begin{array}{llllll}43 & 47.8 & 66 & 09.5 & 26 & \text { Dec. } ' 57\end{array}$

$\begin{array}{lllllll}37563 \mathrm{~B} & 43 & 22.5 & 68 & 00.0 & 9 & " \\ 37564 \mathrm{~B} & 43 & 22.5 & 68 & 00.0 & 9 & "\end{array}$

$43 \quad 48$

$66 \quad 08$

2 Dec. ' 57

$\begin{array}{lllllll}43 & 40.8 & 68 & 00.0 & 9 & \end{array}$

No Returns

$\begin{array}{lllllll}43 & 41.0 & 67 & 00.0 & 9 & \text { " " " }\end{array}$

$\begin{array}{lllllllllll}43 & 40.8 & 66 & 11.0 & 9 & \text { " " }\end{array}$

$\begin{array}{llllll}43 & 40.0 & 66 & 15.0 & 15 \quad "\end{array}$

St. Marys Bay 5 Nov. ' 57 No Returns

$\begin{array}{lllllll}43 & 07.8 & 66 & 44.5 & 16 & " 1\end{array}$

$4251.0 \quad 66 \cdot 59.0 \quad 16 \quad "$ 
Harengus Cruise 18 (cont'd)

Released

Recovered

No.

Bottles

Sta.\# Released Bottle \# Lat. Long. Date

Lat. Iong. Date

\begin{tabular}{|c|c|c|c|c|c|c|c|c|}
\hline 100 & 12 & & 42 & 34.0 & 67 & 13.5 & 16 & Oct. \\
\hline 101 & 12 & & 42 & 25.8 & 67 & 21.0 & 16 & $"$ \\
\hline 102 & 12 & & 42 & 17.2 & 67 & 28.0 & 16 & " \\
\hline 103 & 12 & & 42 & 09.0 & 67 & 34.8 & 16 & H \\
\hline 104 & 12 & & 42 & 04.0 & 67 & 23.5 & 16 & n \\
\hline 105 & 12 & & 42 & 12.0 & 67 & 16.0 & 16 & $"$ \\
\hline 106 & 12 & & 42 & 07.0 & 67 & 05.0 & 16 & $"$ \\
\hline 107 & 12 & & 42 & 15.5 & 66 & 57.2 & 16 & $"$ \\
\hline 108 & 12 & & 42 & 10.5 & 66 & 46.8 & 16 & $"$ \\
\hline 109 & 12 & & 42 & 06.0 & 66 & 32.5 & 16 & $"$ \\
\hline 110 & 12 & & 42 & 13.0 & 66 & 28.0 & 16 & $"$ \\
\hline 111 & 12 & $38266 \mathrm{~B}$ & 42 & 23.0 & 66 & 26.0 & 16 & $"$ \\
\hline 112 & 12 & 3827 I B & 42 & 32.8 & 66 & 23.8 & 16 & $"$ \\
\hline 113 & 12 & & 42 & 42.5 & 66 & 22.2 & 17 & $"$ \\
\hline 114 & 12 & $38080 \mathrm{~N}$ & 43 & 02.5 & 66 & 18.5 & 17 & $"$ \\
\hline 115 & 12 & & 43 & 22.2 & 66 & 14.5 & 17 & $"$ \\
\hline 116 & 12 & $38089 \mathrm{~N}$ & 43 & 40.8 & 66 & 11 & 17 & $"$ \\
\hline 36 & 12 & $38093 \mathrm{~N}$ & 43 & 40.5 & 66 & 10.8 & 22 & $"$ \\
\hline 35 & 12 & & 43 & 51.2 & 66 & 45.5 & 22 & $"$ \\
\hline 34 & 12 & $38109 \mathrm{~N}$ & 44 & 07.2 & 66 & 21 & 22 & $"$ \\
\hline \multirow[t]{2}{*}{33} & 12 & $38114 \mathrm{~N}$ & 44 & 17.0 & 66 & 44.0 & 22 & $"$ \\
\hline & & $38319 \mathrm{~B}$ & 44 & 17.0 & 66 & 44.0 & 22 & $"$ \\
\hline 32 & 12 & $38123 \mathrm{~N}$ & 44 & 40.2 & 65 & 55.5 & 22 & $"$ \\
\hline 31 & 6 & & 44 & 46.5 & 66 & 03.8 & 23 & $"$ \\
\hline 30 & 6 & & 44 & 49.2 & 66 & 08.2 & 23 & $"$ \\
\hline 29 & 6 & & 44 & 52.2 & 66 & 13.0 & 23 & " \\
\hline 28 & 6 & & 44 & 55.5 & 66 & 17.8 & 23 & $"$ \\
\hline 27 & 6 & & 44 & 58.5 & 66 & 22.8 & 23 & $"$ \\
\hline \multirow[t]{3}{*}{26} & 12 & $38358 \mathrm{~B}$ & 45 & 01.0 & 66 & 26.8 & 23 & $"$ \\
\hline & & 38359 B & 45 & 01.0 & 66 & 26.8 & 23 & $"$ \\
\hline & & $38360 \mathrm{~B}$ & 45 & 01.0 & 66 & 26.8 & 23 & $"$ \\
\hline 25 & 6 & $38369 \mathrm{~B}$ & 45 & 10.0 & 65 & 57.3 & 23 & $"$ \\
\hline 24 & 6 & 3837 I B & 45 & 06.8 & 65 & 53.5 & 23 & 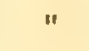 \\
\hline
\end{tabular}

$\begin{array}{lllll}44 & 38.6 & 65 & 39 & 23 \text { Dec. } 157\end{array}$

$44 \quad 22$

6606

4 Dec. 57

No Returns

$\begin{array}{llllll}43 & 26 & 65 & 38 & 14 & \text { Dec。 }\end{array}$

No Returns

$\begin{array}{llllll}44 & 23.5 & 66 & 12.5 & 25 & \text { Oct. '57 }\end{array}$

\begin{tabular}{lllll}
43 & 50 & 66 & 07.5 & $22 \mathrm{Jan}$. \\
\hline
\end{tabular} No Returns

$\begin{array}{llllll}44 & 23.5 & 66 & 12.5 & 25 & \text { Oct. ' } 57\end{array}$

$\begin{array}{llllll}43 & 29.5 & 65 & 43.5 & 1 \mathrm{Jan} . & \text { '58 }\end{array}$

$\begin{array}{llllll}43 & 27 & 65 & 35 & 23 \mathrm{Dec} . & \end{array}$

$\begin{array}{lllll}44 & 36 & 65 & 45 & 18 \text { Nov. '57 }\end{array}$

No Returns

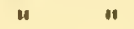

"

11

$\begin{array}{llllll}45 & 05 & 66 & 28 & 13 & \text { Nov. ' } 57\end{array}$

$\begin{array}{lllll}45 & 05 & 66 & 28 & 9\end{array}$

$\begin{array}{lllll}45 & 05.2 & 66 & 28 & 7 \text { Nov. ' } 57\end{array}$

$\begin{array}{llllll}43 & 31 & 65 & 37 & 27 & \mathrm{Dec} .\end{array}$

$\begin{array}{llllll}44 & 36.8 & 65 & 55.4 & 29 & \mathrm{Dec} .\end{array}$ 
Released

No.

Bottles

Sta.\# Released

\begin{tabular}{|c|c|c|c|c|c|c|c|c|}
\hline 23 & 6 & & 45 & 03.8 & 65 & 50.0 & 24 & Oct. \\
\hline 22 & 6 & $38384 \mathrm{~B}$ & 45 & 00.8 & 65 & 46.5 & 24 & $"$ \\
\hline 21 & 6 & & 44 & 57.5 & 65 & 42.5 & 24 & "1 \\
\hline 20 & 6 & & 44 & 54.2 & 65 & 38.8 & 24 & II \\
\hline 19 & 6 & & 44 & 51.0 & 65 & 35.2 & 24 & . \\
\hline 18 & 12 & & 44 & 59.2 & 65 & 17.5 & 24 & . \\
\hline 17 & 12 & & 45 & 06.0 & 65 & 03.0 & 24 & \\
\hline 16 & 6 & & 45 & 09.0 & 65 & 07.0 & 24 & " \\
\hline 15 & 6 & $38424 \mathrm{~B}$ & 45 & 12.5 & 65 & 11.0 & 24 &  \\
\hline 14 & 12 & & 45 & 15.5 & 65 & 15.2 & 24 & 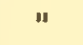 \\
\hline 13 & 6 & & 45 & 18.8 & 65 & 19.2 & 24 & " \\
\hline 12 & 12 & 38447 B & 45 & 22.0 & 65 & 25.2 & 24 & u \\
\hline 11 & 6 & 38452 B & 44 & 43.2 & 65 & 58.8 & 24 & " \\
\hline 10 & 12 & $38163 \mathrm{~N}$ & 44 & 18.8 & 66 & 21.2 & 24 & " \\
\hline 9 & 6 & & 44 & 22.2 & 66 & 23.8 & 25 & " \\
\hline 8 & 6 & & 44 & 26.8 & 66 & 26.8 & 25 & $"$ \\
\hline 7 & 6 & & 44 & 31.2 & 66 & 29.8 & 25 & . \\
\hline 6 & 6 & & 44 & 35.8 & 66 & 33.0 & 25 & 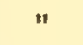 \\
\hline 5 & 6 & & 44 & 40.0 & 66 & 36.0 & 25 & " \\
\hline 4 & 6 & & 44 & 44.8 & 66 & 38.8 & 25 & $"$ \\
\hline 3 & 6 & & 44 & 49.0 & 66 & 42.0 & 25 & " \\
\hline 2 & 12 & & 44 & 53.5 & 66 & 45.0 & 25 & $"$ \\
\hline 1 & 12 & & 44 & 59.7 & 66 & 49.5 & 25 & $"$ \\
\hline
\end{tabular}

Recovered

Long. Date Lat. Long. Date

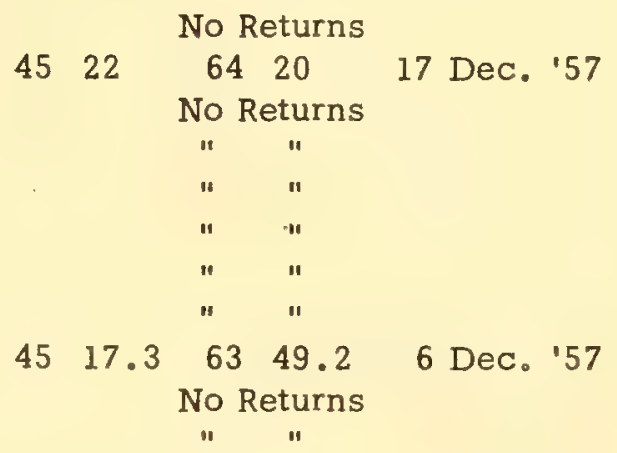

$\begin{array}{llllll}44 & 30 & 66 & 15 & 8 & \text { Dec. } \\ \text { '57 }\end{array}$

$\begin{array}{llllll}44 & 41.5 & 65 & 47 & 30 & \text { Oct. ' } 57\end{array}$

$\begin{array}{lllll}45 & 14 & 64 & 31.5 & 2 \\ & \text { Dec. ' } 57\end{array}$

No Returns

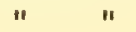

II

II 11

11 1

II

11

I1

II II 
Albatros S III Cruise 104

\section{9- 17 October}

1957

Odd numbered bottles - ballasted, even numbered - no ballast.

Released

Recovered

No.

Bottles

Sta.\# Released Bottle \# Lat. Long。

Date Lat. Long. Date

\begin{tabular}{|c|c|c|c|c|c|c|c|}
\hline 24 & & 41 & 31 & 69 & 32 & 9 & Oct. \\
\hline 12 & & 41 & 30 & 69 & 26 & 9 & " \\
\hline 12 & & 41 & 32 & 69 & 24 & 10 & 18 \\
\hline 10 & & 41 & 29 & 69 & 12.5 & 11 & . \\
\hline 14 & & 41 & 35 & 68 & 58 & 11 & " \\
\hline 15 & & 41 & 37 & 68 & 54 & 12 & $"$ \\
\hline 17 & & 41 & 31 & 68 & 30 & 12 & " \\
\hline 18 & 33534 & 41 & 35 & 68 & 28 & 12 & " \\
\hline 19 & & 41 & 48 & 68 & 15 & 12 & " \\
\hline 12 & & 42 & 02 & 67 & 42 & 12 & $"$ \\
\hline 23 & & 42 & 05 & 67 & 38 & 12 & 11 \\
\hline 24 & & 42 & 08 & 67 & 26 & 12 & " \\
\hline 25 & & 42 & 08 & 67 & 04 & 12 & " \\
\hline 28 & & 41 & 58 & 66 & 18 & 14 & " \\
\hline 32 & & 42 & 20 & 65 & 42 & 14 & $"$ \\
\hline 33 & 33630 & 42 & 45 & 65 & 59 & 14 & $"$ \\
\hline & 33639 & 42 & 45 & 65 & 59 & 14 & " \\
\hline 44 & & 41 & 29 & 67 & 01 & 16 & " \\
\hline 52 & & 40 & 54 & 66 & 45 & 16 & $"$ \\
\hline 60 & & 41 & 49 & 67 & 54 & 17 & $"$ \\
\hline 61 & & 42 & 06 & 67 & 39 & 17 & $"$ \\
\hline 66 & & 41 & 22 & 67 & 51 & 17 & " \\
\hline
\end{tabular}

$\begin{array}{llllllll}68 & 25 & 15 & 56 & \mathrm{E} & 6 \mathrm{Feb} \cdot & \end{array}$ No Returns

" "

"

"

"

"

"

$\begin{array}{llllll}44 & 38 & 65 & 41 & 19 & \text { Jan. '58 }\end{array}$

$\begin{array}{lllll}44 & 23 & 66 & 04 & 30 \text { Dec. } ' 57\end{array}$

No Returns 


\section{Albatross III Cruise 105}

\section{October - 8 November}

\section{7}

12 bottles dropped at each station, odd numbers - ballasted, even numbers - no ballast.

Released

\begin{tabular}{|c|c|c|c|c|c|c|c|}
\hline Sta.\# & Bottle \# & & at. & & $\mathrm{ng}$ 。 & & ate \\
\hline 20 & 33702 & 42 & 51 & 66 & 18 & 29 & Oct. \\
\hline & 33705 & 42 & 51 & 66 & 18 & 29 & $"$ \\
\hline 21 & 33712 & 42 & 51 & 65 & 57 & 30 & $"$ \\
\hline 23 & 33726 & 43 & 15 & 66 & 23 & 30 & $"$ \\
\hline 25 & & 43 & 31 & 66 & 26 & 30 & $"$ \\
\hline 27 & 33753 & 43 & 35 & 66 & 27 & 30 & $"$ \\
\hline 31 & 33761 & 44 & 09 & 67 & 06 & 31 & $"$ \\
\hline & 33762 & 44 & 09 & 67 & 06 & 31 & $"$ \\
\hline 32 & 33772 & 44 & 08 & 67 & 04 & 1 & Nov. \\
\hline & 33778 & 44 & 08 & 67 & 04 & 1 & $"$ \\
\hline 33 & 33786 & 44 & 01 & 67 & 18 & 1 & $"$ \\
\hline 35 & & 44 & 09 & 67 & 53 & 1 & $"$ \\
\hline 37 & 33806 & 43 & 47 & 68 & 13 & 1 & $"$ \\
\hline 39 & 33826 & 43 & 43 & 68 & 36 & 2 & $"$ \\
\hline 40 & 33832 & 43 & 35 & 68 & 39 & 2 & $"$ \\
\hline $\mathrm{X}$ & & 42 & 56 & 68 & 58 & 2 & $"$ \\
\hline 47 & & 43 & 40 & 68 & 59 & 5 & $"$ \\
\hline 49 & 33867 & 43 & 37 & 68 & 35 & 5 & $"$ \\
\hline 51 & & 43 & 44 & 68 & 11 & 5 & $"$ \\
\hline 53 & 33889 & 43 & 54 & 67 & 43 & 5 & $"$ \\
\hline 55 & & 44 & 03 & 67 & 20 & 5 & $"$ \\
\hline 57 & 33913 & 44 & 11 & 66 & 53 & 5 & $"$ \\
\hline & 33919 & 44 & 11 & 66 & 53 & 5 & $"$ \\
\hline 59 & 33933 & 44 & 15 & 66 & 35 & 5 & $"$ \\
\hline 60 & & 44 & 24 & 66 & 22 & 6 & $"$ \\
\hline 79 & 33950 & 43 & 10 & 69 & 22 & 7 & " \\
\hline 82 & & 42 & 51 & 69 & 23 & 7 & $"$ \\
\hline 88 & & 42 & 23 & 70 & 11 & 7 & $"$ \\
\hline 89 & & 42 & 04 & 69 & 53 & 8 & $"$ \\
\hline
\end{tabular}

Recovered

Lat. Long。 Date

$\begin{array}{llllll}44 & 30 & 66 & 06 & 21 \mathrm{Jan} . & \end{array}$

$\begin{array}{llllll}44 & 54.5 & 65 & 21 & 28 \text { Nov. '57 }\end{array}$

$\begin{array}{lllll}44 & 26 & 66 & 02 & 26 \\ 40 v & \text { ' } 57\end{array}$

\begin{tabular}{llllll}
44 & 34 & 65 & 54 & 24 & Dec. \\
\hline & 57
\end{tabular}

No Returns

$\begin{array}{llllll}44 & 11.5 & 66 & 10 & 26 & \text { Dec. } ' 57\end{array}$

\begin{tabular}{lllll}
43 & 53.3 & 66 & 09.8 & 3 \\
\hline Dec. & 57
\end{tabular}

$\begin{array}{llllll}44 & 10 & 66 & 10 & 18 & \mathrm{Dec} . \\ & 57\end{array}$

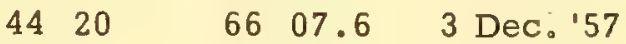

$\begin{array}{lllll}44 & 02 & 66 & 10 & 1\end{array}$ Dec. ' 57

$\begin{array}{llllll}44 & 03 & 66 & 10.2 & 29 & \text { Dec. '57 }\end{array}$

No Returns

$\begin{array}{llllll}45 & 05.4 & 66 & 55.0 & 27 & \text { Aug. '58 }\end{array}$

$\begin{array}{llllll}41 & 42.5 & 70 & 20 & 20 & \text { Mar. } 159\end{array}$

$4143.7 \quad 70 \quad 14 \quad 10$ Apr. '60

No Returns

$\begin{array}{llllll}43 & 59 & 66 & 09.3 & 13 & \text { Dec. ' } 57\end{array}$

No Returns

$\begin{array}{llllll}44 & 18 & 66 & 07.5 & 2 & \text { Dec. } ' 57\end{array}$

No Returns

$\begin{array}{llllll}43 & 59 & 66 & 09.3 & 3 & \text { Dec. } 57\end{array}$

$\begin{array}{llllll}44 & 18 & 66 & 08 & 7 & \text { Dec。' } 57\end{array}$

$\begin{array}{lllll}44 & 53 & 65 & 24 & 13 \\ \text { Nov. } & 57\end{array}$

No Returns

$\begin{array}{llllll}42 & 04.2 & 70 & 14.6 & 10 & \text { Apr。 } 58\end{array}$

No Returns 
Harengus Cruise 19

5 - 21 November

1957

At stations where 12 bottles were dropped, 6 were ballasted, 6 were without ballast. At stations where 6 bottles were dropped, all were ballasted.

Released

Recovered

No.

Bottles

Sta.\# Released Bottle \# Lat. Long. Date Lat. Long. Date

\begin{tabular}{|c|c|c|c|c|c|c|c|c|c|c|c|c|c|}
\hline 95 & 12 & $38514 \mathrm{~B}$ & 44 & 57.5 & 66 & 51.2 & 5 & Nov. & 44 & 46.5 & 66 & 49.4 & 7 Nov. \\
\hline & & 38515 B & 44 & 57.5 & 66 & 51.2 & 5 & $"$ & 45 & 00.2 & 66 & 56.7 & 17 Nov. \\
\hline 94 & 12 & $38990 \mathrm{~N}$ & 44 & 32.0 & 67 & 12.0 & 5 & $"$ & 43 & 31.5 & 65 & 44 & 4 Dec. \\
\hline & & $38994 \mathrm{~N}$ & 44 & 32.0 & 67 & 12.0 & 5 & $"$ & 44 & 33 & 66 & 02 & $30 \mathrm{Dec}$ \\
\hline 93 & 12 & $38996 \mathrm{~N}$ & 44 & 24.0 & 67 & 44.0 & 5 & $"$ & 43 & 27.5 & 65 & 39.3 & $14 \mathrm{Dec}$. \\
\hline 2 & 12 & 38535 B & 44 & 00.0 & 67 & 39.0 & 5 & $"$ & 44 & 30 & 66 & 06 & 13 Jan. \\
\hline & & $39003 \mathrm{~N}$ & 44 & 00.0 & 67 & 39.0 & 5 & " & 43 & 28.9 & 65 & 33.5 & 22 Dec. \\
\hline & & $39005 \mathrm{~N}$ & 44 & 00.0 & 67 & 39.0 & 5 & $"$ & 44 & 13 & 66 & 09 & 6 Jan. \\
\hline 1 & 12 & & 44 & 00.0 & 68 & 34.0 & 5 & $"$ & & & No $R$ & eturns & \\
\hline 30 & 12 & & 43 & 40.0 & 68 & 36.0 & 6 & $"$ & & & " & $"$ & \\
\hline 89 & 12 & & 43 & 41.5 & 69 & 01.0 & 6 & $"$ & & & $"$ & $"$ & \\
\hline 38 & 12 & & 43 & 22.0 & 70 & 20.0 & 6 & $"$ & & & $"$ & $"$ & \\
\hline 87 & 12 & $39035 \mathrm{~N}$ & 43 & 00.0 & 69 & 56.0 & 6 & $"$ & 62 & 30 & 08 & $20 \mathrm{E}$ & 3 Aug. \\
\hline 6 & 12 & $38605 \mathrm{~B}$ & 43 & 00.0 & 69 & 00.0 & 6 & $"$ & 43 & 31 & 65 & 38 & 6 Jan. \\
\hline & & $38609 \mathrm{~B}$ & 43 & 00.0 & 69 & 00.0 & 6 & $"$ & 43 & 29 & 65 & 40 & 9 Jan. \\
\hline & & $38610 \mathrm{~B}$ & 43 & 00.0 & 69 & 00.0 & 6 & $"$ & 43 & 32.3 & 65 & 47.5 & 1 Jan. \\
\hline & & $39039 \mathrm{~N}$ & 43 & 00.0 & 69 & 00.0 & 6 & $"$ & 43 & 25 & 66 & 01 & 14 Aug. \\
\hline 85 & 12 & $38616 \mathrm{~B}$ & 43 & 22.0 & 69 & 00.0 & 6 & $"$ & 41 & 17 & 70 & 14.5 & $5 \mathrm{Apr}$. \\
\hline 84 & 12 & $39054 \mathrm{~N}$ & 43 & 22.5 & 67 & 58.0 & 7 & $"$ & 44 & 22 & 66 & 06 & $31 \mathrm{Mar}$. \\
\hline 83 & 12 & $38623 \mathrm{~B}$ & 43 & 41.0 & 67 & 57.0 & 7 & $"$ & 43 & 32 & 65 & 45.5 & 9 Jan. \\
\hline & & $39055 \mathrm{~N}$ & 43 & 41.0 & 67 & 57.0 & 7 & $"$ & 43 & 47 & 66 & 08 & I Dec. \\
\hline & & $39057 \mathrm{~N}$ & 43 & 41.0 & 67 & 57.0 & 7 & $"$ & 44 & 13.2 & 66 & 08.6 & $4 \mathrm{Dec}$ \\
\hline 82 & 12 & & 43 & 41.0 & 67 & 01.0 & 7 & $"$ & & & No $R$ & eturns & \\
\hline 35 & 12 & & 43 & 51.0 & 66 & 45.0 & 12 & $"$ & & & $"$ & $"$ & \\
\hline 96 & 12 & $38643 \mathrm{~B}$ & 43 & 40.0 & 66 & 15.0 & 12 & " & 44 & 30.5 & 65 & 55 & 6 Dec. \\
\hline 97 & 12 & $39083 \mathrm{~N}$ & 43 & 24 & 66 & 22 & 12 & $"$ & 44 & 55 & 65 & 21 & 26 Nov. \\
\hline 98 & 12 & & 43 & 07 & 66 & 45 & 12 & " & & & No $R$ & eturns & \\
\hline
\end{tabular}


Harengus Cruise 19 (cont'd)

Released

Recovered

No.

Bottles

Sta.\# Released

Bottle \# Lat. Long. Date Lat. Long. Date

99

$12 \quad 39096 \mathrm{~N} \quad 42 \quad 51$

$66 \quad 59 \quad 12$ Nov.

$\begin{array}{lllll}44 & 03 & 66 & 10 & 27 \text { Nov. ' } 57\end{array}$

$\begin{array}{lllllllllllll}38659 & \text { B } & 42 & 51 & 66 & 59 & 12 & \text { " } & 44 & 23.7 & 66 & 12 & 26 \\ 3 & \text { Nov. ' } 57\end{array}$

\begin{tabular}{lllllllllllll}
38661 & B & 42 & 51 & 66 & 59 & 12 & $"$ & 43 & 37.5 & 66 & 03.5 & 4 \\
\hline
\end{tabular}

100

39101 B $42 \quad 34.5$

$67 \quad 14$

$58 \quad 15$

$06 \quad 46 \quad 12$ Aug. '59

$\begin{array}{lllllllllll}39102 & N & 42 & 34.5 & 67 & 14 & 12 & \text { " }\end{array}$

62

06

29 Apr. ' 59

$\begin{array}{llllllll}42 & 25.5 & 67 & 21 & 12 & \text { " }\end{array}$

$\begin{array}{ll}101 & 12 \\ 102 & 12\end{array}$

$42 \quad 17$

$\begin{array}{ll}67 & 20\end{array}$

No Returns

$103 \quad 12$

$\begin{array}{ll}42 & 09\end{array}$

$\begin{array}{llll}67 & 34.5 & 13\end{array}$

$104 \quad 12$

$42 \quad 04$

$67 \quad 23$
67

" "

$105 \quad 12$

$42 \quad 12$
42

$67 \quad 16$

"

n

$\begin{array}{ll}42 & 08\end{array}$

$\begin{array}{ll}67 & 00\end{array}$

a

$42 \quad 15$

$\begin{array}{ll}66 & 58\end{array}$

"

$\begin{array}{llllll}42 & 10 & 66 & 47 & 13\end{array}$

a

ห

$\begin{array}{lllllll}42 & 04.5 & 66 & 35 & 13 & \text { " }\end{array}$

$\begin{array}{llllll}42 & 13 & 66 & 26 & 13 & \text { * }\end{array}$

$110 \quad 12$

$111 \quad 12$

$42 \quad 22$

$\begin{array}{ll}66 & 28\end{array}$

"

"

$112 \quad 12$

$39169 \mathrm{~N} \quad 42 \quad 34$

$\begin{array}{ll}66 & 25\end{array}$

$"$

$\begin{array}{llll}43 & 24 & 65 & 37\end{array}$

11 Dec. ' 57

$39172 \mathrm{~N}$

$42 \quad 34$

$\begin{array}{ll}66 & 25\end{array}$

$\begin{array}{ll}66 & 07.6\end{array}$

4 Dec. '57

$\begin{array}{llll}42 & 44 & 66 & 22\end{array}$

$44 \quad 20$

No Returns

$\begin{array}{llllll}44 & 37 & 65 & 42 & 1\end{array}$

$\begin{array}{llllllll}38753 & \text { B } & 43 & 05 & 66 & 18 & 13 & \text { \% }\end{array}$

$\begin{array}{llll}66 & 14.5 & 13 & \end{array}$

$\begin{array}{lll}43 & 47\end{array}$

6608

25 Nov. ' 57

$\begin{array}{llllll}44 & 06.5 & 66 & 21 & 15\end{array}$

No Returns

$\begin{array}{llllll}44 & 30 & 66 & 15 & 4 & \text { Dec. '57 }\end{array}$

No Returns

$\begin{array}{llllll}45 & 00.5 & 65 & 09 & 2 \\ \text { Dec. '57 }\end{array}$

No Returns

$\begin{array}{llllll}44 & 52.5 & 66 & 13 & 18\end{array}$

" n

$\begin{array}{lllllll}44 & 55.2 & 66 & 17.3 & 18 & \text { " }\end{array}$

" $"$

$\begin{array}{lllll}45 & 05 & 66 & 28 & 25 \text { Nov. '57 }\end{array}$

$\begin{array}{lllll}45 & 04.5 & 66 & 44.4 & 23 \\ 4 & \text { Nov. ' } 57\end{array}$

$\begin{array}{llllll}45 & 04.5 & 66 & 44.4 & 13 \\ & \text { Jan. '58 }\end{array}$

$\begin{array}{llllll}45 & 07 & 66 & 21 & 7 & \text { Mar. '58 }\end{array}$

No Returns

$\begin{array}{llllll}45 & 06.8 & 65 & 53.5 & 18 & 18\end{array}$

$\begin{array}{lllllll}45 & 03.8 & 65 & 50 & 18\end{array}$ 
Harengus Cruise 19 (cont'd)

Released

Recovered

No.

Bottles

Sta.\# Reléased

$\begin{array}{rrllllllll}22 & 12 & 38838 \text { B } & 45 & 00.8 & 65 & 46.5 & 18 \text { Nov. } \\ 21 & 6 & & 44 & 57.5 & 65 & 42.5 & 18 & " \\ 20 & 6 & & 44 & 54.2 & 65 & 38.8 & 18 & " \\ 19 & 6 & & 44 & 51 & 65 & 35.2 & 18 & " \\ 18 & 12 & & 44 & 59.2 & 65 & 17.5 & 18 & " \\ 17 & 12 & 38863 \text { B } & 45 & 09 & 64 & 59 & 18 & " \\ & & 38867 \text { B } & 45 & 09 & 64 & 59 & 18 & " \\ 16 & 6 & & 45 & 09 & 65 & 07 & 18 & " \\ 15 & 6 & & 45 & 12.5 & 65 & 11 & 18 & " \\ 14 & 12 & 39240 \mathrm{~N} & 45 & 15.5 & 65 & 15.2 & 19 & " \\ 13 & 6 & 38892 \mathrm{~B} & 45 & 18.5 & 65 & 19 & 19 & " \\ 12 & 12 & 38894 \mathrm{~B} & 45 & 21.8 & 65 & 23 & 19 & " \\ & & 39246 \mathrm{~N} & 45 & 21.8 & 65 & 23 & 19 & " \\ 11 & 6 & & 44 & 43.2 & 65 & 58.8 & 21 & " \\ 10 & 12 & & 44 & 18.8 & 66 & 21.2 & 21 & " \\ 9 & 6 & & 44 & 22.5 & 66 & 23.8 & 21 & " \\ 8 & 6 & & 44 & 27 & 66 & 26.8 & 21 & " \\ 7 & 6 & 38927 \mathrm{~B} & 44 & 31.5 & 66 & 29.8 & 21 & " \\ 6 & 12 & & 44 & 35.8 & 66 & 32.8 & 21 & " \\ 5 & 6 & & 44 & 40 & 66 & 36 & 21 & " \\ 4 & 6 & & 44 & 44.5 & 66 & 39 & 21 & " \\ 3 & 6 & & 44 & 49 & 66 & 42 & 21 & " \\ 2 & 12 & 38953 & 44 & 53.5 & 66 & 45 & 21 & " \\ 1 & 12 & & 44 & 59.5 & 66 & 49.2 & 21 & "\end{array}$

$44 \quad 57.5^{\prime} 65 \quad 14.5^{\prime} 30$ Nov. '57 No Returns

$\begin{array}{llllll}45 & 18.5 & 64 & 45 & 25 & \text { Nov. '57 }\end{array}$

$\begin{array}{lllll}45 & 13 & 64 & 31.3 & 27 \\ \text { Nov。' } 57\end{array}$

No Returns

$\begin{array}{llllll}45 & 18.4 & 64 & 47.3 & 5 & \text { Mar. '58 }\end{array}$

$\begin{array}{llllll}45 & 19.5 & 64 & 46,5 & 27 & \text { Nov. ' } 57\end{array}$

$\begin{array}{lllll}45 & 20 & 64 & 46.5 & 29 \text { Nov. '57 }\end{array}$

$\begin{array}{llllll}45 & 22.5 & 64 & 41.2 & 20 & \text { Dec. '57 }\end{array}$

No Returns

"

14

" 1

$\begin{array}{llllll}44 & 40 & 65 & 46 & 28 & \text { Nov. '57 }\end{array}$

No Returns

" "

"

"

$44 \quad 30 \quad 66 \quad 06 \quad 31$ Dec. '57 No Returns 
Albatross III Cruise 106

3- 19 December

1957

All bottles ballasted.

Released

Recovered

No.

Bottles

Sta.\# Released Bottle \# Lat. Long. Date Lat. Long. Date

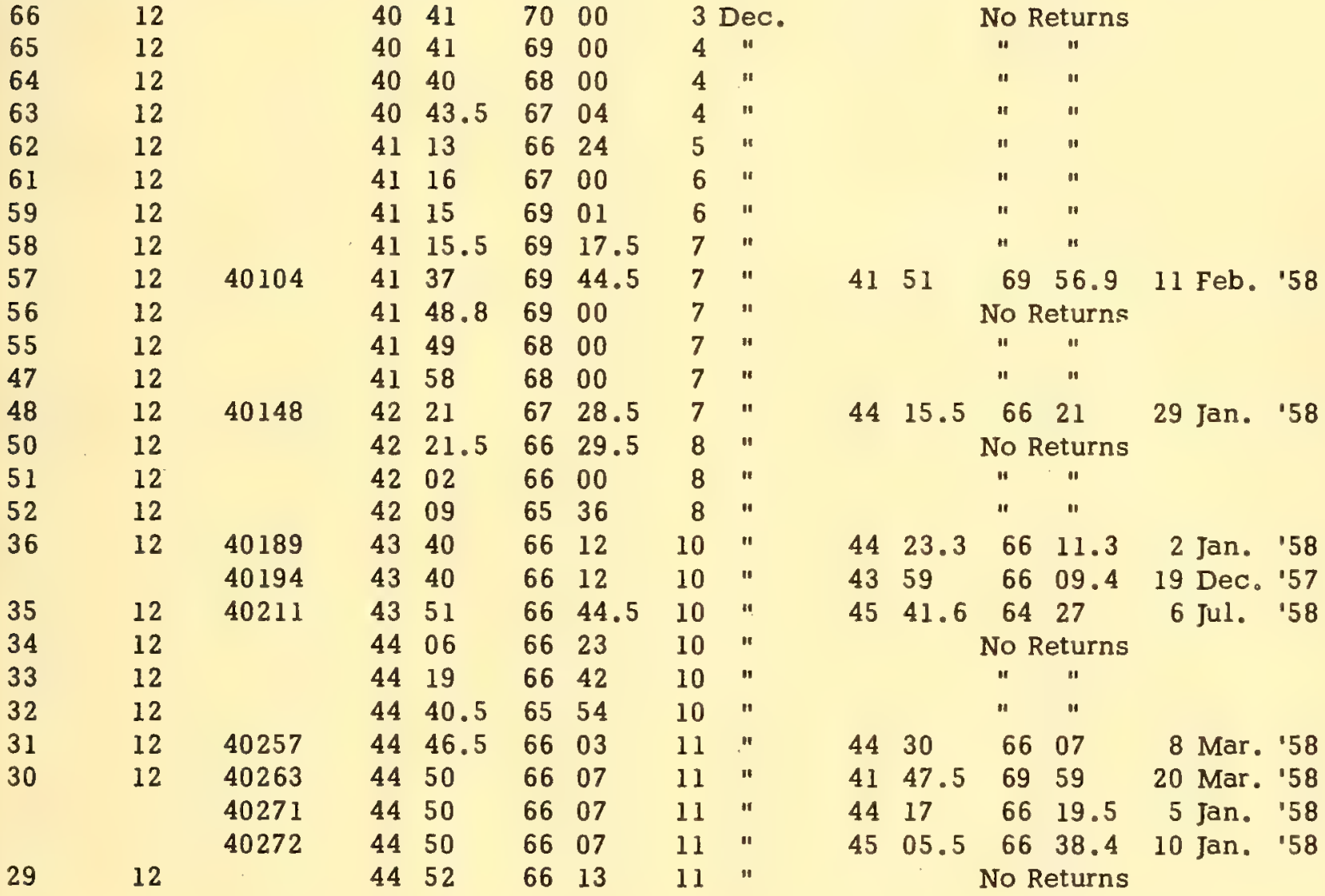


Released

Recovered

No.

Bottles

Sta.\# Released Bottle \# Lat. Long. Date Lat. Long. Date

\begin{tabular}{|c|c|c|c|c|c|c|c|c|c|c|c|c|c|c|}
\hline \multirow[t]{3}{*}{28} & 12 & 40285 & 44 & 54 & 66 & 19 & 11 & Dec. & 44 & 55 & 65 & 20.5 & $25 \mathrm{Jan}$. & '58 \\
\hline & & 40290 & 44 & 54 & 66 & 19 & 11 & $"$ & 44 & 24 & 66 & 13 & 7 Jan. & $' 58$ \\
\hline & & 40292 & 44 & 54 & 66 & 19 & 11 & $"$ & 45 & 13 & 66 & 07.5 & 2 Jan. & '58 \\
\hline \multirow[t]{2}{*}{2.7} & 12 & 40303 & 44 & 57.5 & 66 & 26 & 11 & $"$ & 45 & 03.4 & 66 & 47.7 & 2 Jan. & '58 \\
\hline & & 40304 & 44 & 57.5 & 66 & 26 & 11 & " & 45 & 00.8 & 66 & 54 & 20 Dec. & "57 \\
\hline \multirow[t]{3}{*}{26} & 12 & 40311 & 45 & 01.5 & 66 & 27 & 11 & $"$ & 44 & 48.5 & 66 & 58.7 & 7 Jan. & '58 \\
\hline & & 40314 & 45 & 01.5 & 66 & 27 & 11 & $"$ & 45 & 05.8 & 66 & 38.6 & i4 Jan. & '58 \\
\hline & & 40317 & 45 & 01.5 & 66 & 27 & 11 & $"$ & 45 & 01 & 66 & 51 & $20 \mathrm{Dec}$. & 157 \\
\hline 25 & 12 & 40327 & 45 & 10 & 65 & 57 & 11 & $"$ & 45 & 15 & 66 & 04 & 25 Dec. & ' 57 \\
\hline 24 & 12 & & 45 & 06 & 65 & 55 & 11 & $"$ & \multicolumn{6}{|c|}{ No Returns } \\
\hline \multirow[t]{2}{*}{23} & 12 & 40352 & 45 & 04 & 65 & 49 & 11 & $"$ & 45 & 20 & 64 & 46.5 & 29 Dec. & 157 \\
\hline & & 40354 & 45 & 04 & 65 & 49 & 11 & $"$ & 42 & 04.8 & 70 & 13 & 13 May & 58 \\
\hline 22 & 12 & & 45 & 01 & 65 & 47 & 11 & $"$ & \multicolumn{6}{|c|}{ No Returns } \\
\hline 21 & 12 & 40369 & 44 & 57 & 65 & 43 & 11 & $"$ & $45^{\circ}$ & 20.3 & 65 & 47.5 & 4 Aug. & 58 \\
\hline 20 & 12 & & 44 & 54 & 65 & 38 & 11 & $"$ & & & No $R$ & eturns & & \\
\hline 19 & 12 & & 44 & 51 & 65 & 35 & 11 & $"$ & & & $"$ & $"$ & & \\
\hline 18 & 12 & & 44 & 59 & 65 & 19 & 11 & $"$ & & & $"$ & $"$ & & \\
\hline 17 & 12 & 40422 & 45 & 07 & 65 & 04 & 11 & $"$ & 45 & 20 & 64 & 47 & 4 Jan. & ' 58 \\
\hline \multirow[t]{2}{*}{16} & 12 & 40430 & 45 & 10.5 & 65 & 06.5 & 11 & $"$ & 45 & 20 & 64 & 47 & 9 Jan. & 158 \\
\hline & & 40433 & 45 & 10.5 & 65 & 06.5 & 11 & $"$ & 45 & 28.5 & 64 & 51.5 & 27 Dec. & 157 \\
\hline \multirow[t]{2}{*}{15} & 12 & 40442 & 45 & 12.5 & 65 & 11 & 11 & $"$ & 45 & 20 & 64 & 47 & 2 Jan. & '58 \\
\hline & & 40447 & 45 & 12.5 & 65 & 11 & 11 & $"$ & 45 & 20 & 64 & 47 & $10 \mathrm{Jan}$. & 158 \\
\hline \multirow[t]{3}{*}{14} & 12 & 40458 & 45 & 16 & 65 & 14 & 11 & $"$ & 45 & 20 & 64 & 47 & 7 Jan. & 158 \\
\hline & & 40459 & 45 & 16 & 65 & 14 & 11 & $"$ & 45 & 10 & 64 & 46 & 25 Jan. & '58 \\
\hline & & 40522 & 45 & 16 & 65 & 14 & 11 & $"$ & 45 & 23 & 64 & 19 & 20 Feb. & 158 \\
\hline 13 & 12 & 40535 & 45 & 19 & 65 & 18 & 11 & $"$ & 45 & 22 & 64 & 20.3 & 19 Jan. & 158 \\
\hline 12 & 12 & 40545 & 45 & 22 & 65 & 22 & 12 & $"$ & 44 & 26.2 & 66 & 10.8 & $4 \mathrm{Feb}$. & '58 \\
\hline 11 & 12 & 40555 & 44 & 44 & 65 & 59 & 12 & $"$ & 45 & 20 & 64 & 47 & 3 Jan. & $' 58$ \\
\hline 10 & 12 & 40561 & 44 & 19 & 66 & 21 & 12 & $"$ & 45 & 04 & 66 & 42.8 & $30 \mathrm{Dec}$. & 57 \\
\hline 9 & 12 & & 44 & 22 & 66 & 23 & 12 & $"$ & \multicolumn{5}{|c|}{ No Returns } & \\
\hline \multirow[t]{5}{*}{8} & 12 & 40586 & 44 & 27 & 66 & 27 & 12 & $"$ & 45 & 08 & 66 & 21 & 10 Mar. & '58 \\
\hline & & 40588 & 44 & 27 & 66 & 27 & 12 & $"$ & 45 & 11 & 66 & 09 & 8 Apr. & 158 \\
\hline & & 40590 & 44 & 27 & 66 & 27 & 12 & $"$ & 44 & 17 & 66 & 20 & 12 Jan. & 158 \\
\hline & & 40594 & 44 & 27 & 66 & 27 & 12 & $"$ & 45 & 04.5 & 66 & 43.8 & 20 Apr. & '58 \\
\hline & & 40596 & 44 & 27 & 66 & 27 & 12 & $"$ & 45 & 03.8 & 66 & 43.9 & 27 Dec. & '57 \\
\hline
\end{tabular}


Released

Recovered

No.

Bottles

Sta.\# Released Bottle \# Lat. Long. Date Lat. Long. Date




Delaware 57-9

17 - 19 December

1957

All bottles ballasted.

Released

Recovered

No.

Bottles

\begin{tabular}{|c|c|c|c|c|c|c|c|c|c|c|c|c|c|}
\hline \multirow{2}{*}{$\begin{array}{c}\text { Sta.\# } \\
1\end{array}$} & \multirow{2}{*}{ Released } & \multirow{2}{*}{$\begin{array}{l}\text { Bottle \# } \\
41171\end{array}$} & \multicolumn{2}{|c|}{ Lat. } & \multicolumn{2}{|c|}{ Long. } & \multicolumn{2}{|c|}{ Date } & \multicolumn{2}{|c|}{ Lat. } & Long. & \multicolumn{2}{|l|}{ Date } \\
\hline & & & 43 & 39 & 66 & 33 & 17 & Dec. & 44 & 26 & $\begin{array}{ll}66 & 10.5\end{array}$ & 6 Jan. & '58 \\
\hline $3+$ & 12 & & 43 & 42 & 67 & 00 & 17 & $"$ & & & No Returns & & \\
\hline $8+$ & 12 & & 43 & 41 & 68 & 00 & 18 & 18 & & & $n$ & & \\
\hline $10+$ & 12 & & 43 & 22 & 68 & 00 & 18 & $"$ & & & $"$ & & \\
\hline $15+$ & 12 & 41217 & 43 & 12 & 67 & 00 & 18 & $"$ & 44 & 16 & $\begin{array}{ll}66 & 19\end{array}$ & 1 Feb. & '58 \\
\hline & & 41209 & 43 & 12 & 67 & 00 & 18 & $"$ & 44 & 17 & $\begin{array}{ll}66 & 19.5\end{array}$ & 5 Jan. & $\cdot 58$ \\
\hline 18 & 12 & & 42 & 41 & 67 & 00 & 18 & $"$ & & & No Returns & & \\
\hline $19+$ & 12 & 41233 & 42 & 30 & 66 & 55 & 18 & " & 41 & 40.2 & $70 \quad 00$ & 21 Aug. & 58 \\
\hline $27+$ & 12 & & 41 & 48 & 65 & 35 & 18 & " & & & No Returns & & \\
\hline 35 & 12 & & 41 & 50 & 69 & 00 & 19 & $"$ & & & " & & \\
\hline
\end{tabular}


Albatross III Cruise 107

8 - 20 January

1958

Some ballasted, som» not.

Released

Recovered

No.

Bottles

Sta.\# Released Bottle \# Lat. Long. Date Lat. Long. Date

\begin{tabular}{|c|c|c|c|c|c|c|c|c|c|c|c|c|}
\hline 66 & 12 & & 40 & 40 & 70 & 00 & \multicolumn{2}{|c|}{8 Jan. } & \multicolumn{4}{|c|}{ No Returns } \\
\hline 65 & 12 & & 40 & 40 & 69 & 00 & 9 & $"$ & & " & 4 & \\
\hline 64 & 12 & & 40 & 40.5 & 68 & 00 & 9 & $"$ & & $"$ & $1 "$ & \\
\hline 63 & 12 & & 40 & 41 & 67 & 00 & 9 & " & & $"$ & $n$ & \\
\hline 62 & 12 & & 41 & 15.5 & 66 & 13 & 10 & $n$ & & $"$ & n & \\
\hline 61 & 12 & & 41 & 16 & 67 & 04.5 & 10 & " & & n & $"$ & \\
\hline 60 & 12 & & 41 & 17 & 68 & 00 & 10 & $"$ & & " & $"$ & \\
\hline 59 & 12 & & 41 & 16 & 69 & 00 & 10 & $"$ & & $"$ & $"$ & \\
\hline 58 & 12 & & 41 & 17 & 69 & 24 & 10 & $"$ & & $"$ & $"$ & \\
\hline 57 & 12 & & 41 & 37 & 69 & 43 & 11 & $"$ & & $"$ & " & \\
\hline 56 & 12 & & 41 & 49 & 69 & 00 & 11 & " & & $"$ & $"$ & \\
\hline 55 & 12 & & 41 & 50 & 68 & 00 & 11 & $"$ & & " & $"$ & \\
\hline $5 \Delta$ & 12 & & 41 & 49 & 67 & 00 & 11 & $"$ & & $"$ & $"$ & \\
\hline 53 & 12 & & 41 & 50 & 65 & 37 & 12 & " & & $"$ & $"$ & \\
\hline 52 & 12 & & 42 & 09 & 65 & 35 & 12 & $"$ & & $"$ & $"$ & \\
\hline 51 & 12 & & 42 & 03 & 66 & 00 & 12 & $"$ & & " & $"$ & \\
\hline 50 & 12 & & 42 & 20 & 66 & 31 & 12 & " & & $"$ & $"$ & \\
\hline 49 & 12 & & 42 & 06 & 67 & 00 & 12 & $"$ & & $"$ & $n$ & \\
\hline 48 & 12 & & 42 & 21 & 67 & 29 & 12 & $"$ & & $"$ & $"$ & \\
\hline 47 & 12 & & 41 & 58 & 68 & 00 & 13 & " & & $"$ & $n$ & \\
\hline 46 & 12 & & 42 & 11 & 68 & 31 & 13 & $"$ & & $"$ & $"$ & \\
\hline 45 & 12 & & 42 & 12 & 69 & 29 & 13 & $n$ & & $"$ & $"$ & \\
\hline 44 & 12 & & 42 & 30 & 69 & 27 & 14 & $"$ & & $\checkmark$ & $"$ & \\
\hline 43 & 12 & & 42 & 30 & 68 & 00 & 14 & $"$ & & n & $"$ & \\
\hline 36 & 12 & & 43 & 40 & 66 & 12 & 17 & $"$ & & $"$ & $"$ & \\
\hline 35 & 12 & $41376 \mathrm{~B}$ & 43 & 51 & 66 & 46 & 18 & $"$ & $41 \quad 27.2$ & 70 & 35.9 & $31 \mathrm{Mar} .58$ \\
\hline 34 & 12 & & 44 & 05.5 & 66 & 22 & 18 & $"$ & & No $R e$ & turns & \\
\hline 33 & 12 & & 44 & 18 & 66 & 43 & 18 & " & & $"$ & '4 & \\
\hline
\end{tabular}


Released

Recovered

No.

Bottles

Sta.\# Released Bottle\# Lat. Long。 Date

\begin{tabular}{|c|c|c|c|c|c|c|c|c|}
\hline 32 & 12 & & 44 & 36.5 & 66 & 18 & 18 & Tan. \\
\hline 31 & 12 & & 44 & 43.5 & 66 & 14 & 18 & $"$ \\
\hline $3 C$ & 12 & $41425 \mathrm{~B}$ & 44 & 49 & 66 & 08 & 18 & $"$ \\
\hline 29 & 12 & & 44 & 52 & 66 & 13 & 18 & $"$ \\
\hline 28 & 12 & & 44 & 55 & 66 & 18 & 18 & $"$ \\
\hline 27 & 12 & & 44 & 58 & 66 & 24 & 18 & $"$ \\
\hline 26 & 12 & & 45 & 01 & 66 & 27 & 18 & $"$ \\
\hline 25 & 12 & $41480 \mathrm{~N}$ & 45 & 10 & 65 & 56 & 18 & $"$ \\
\hline 24 & 12 & $41517 \mathrm{~B}$ & 45 & 06.5 & 65 & 53 & 18 & n \\
\hline 23 & 12 & $41491 \mathrm{~N}$ & 45 & 03 & 65 & 50 & 18 & $"$ \\
\hline 21 & 12 & & 44 & 57 & 65 & 43 & 18 & $"$ \\
\hline 20 & 12 & & 44 & 53.5 & 65 & 39 & 18 & " \\
\hline 18 & 12 & & 44 & 58.5 & 65 & 18 & 18 & " \\
\hline 17 & 12 & & 45 & 06 & 65 & 04 & 18 & $"$ \\
\hline 16 & 11 & & 45 & 09 & 65 & 07 & 18 & $"$ \\
\hline 15 & 12 & & 45 & 12 & 65 & 11 & 18 & * \\
\hline 14 & 12 & & 45 & 16 & 65 & 14 & 18 & $"$ \\
\hline 13 & 12 & $41601 \mathrm{~N}$ & 45 & 19 & 65 & 18 & 18 & $\mu$ \\
\hline \multirow[t]{4}{*}{12} & 12 & $41606 \mathrm{~N}$ & 45 & 22 & 65 & 22 & 18 & $"$ \\
\hline & & $41607 \mathrm{~N}$ & 45 & 22 & 65 & 22 & 18 & " \\
\hline & & $41608 \mathrm{~N}$ & 45 & 22 & 65 & 22 & 18 & " \\
\hline & & $41631 \mathrm{~B}$ & 45 & 22 & 65 & 22 & 18 & $"$ \\
\hline \multirow[t]{2}{*}{10} & 12 & $41496 \mathrm{~N}$ & 44 & 19 & 66 & 21 & 19 & $"$ \\
\hline & & $41667 \mathrm{~N}$ & 44 & 19 & 66 & 21 & 19 & $"$ \\
\hline 9 & 12 & & 44 & 22 & 66 & 23 & 19 & $"$ \\
\hline 8 & 12 & & 44 & 27 & 66 & 25 & 19 & $"$ \\
\hline 7 & 12 & & 44 & 32 & 66 & 31 & 19 & $"$ \\
\hline 6 & 12 & & 44 & 36 & 66 & 34 & 19 & $"$ \\
\hline 5 & 12 & & 44 & 40 & 66 & 36 & 19 & $"$ \\
\hline \multirow[t]{2}{*}{4} & 12 & $41706 \mathrm{~N}$ & 44 & 40 & 66 & 41 & 19 & $"$ \\
\hline & & $41709 \mathrm{~N}$ & 44 & 40 & 66 & 41 & 19 & $"$ \\
\hline 3 & 12 & $41721 \mathrm{~B}$ & 44 & 49 & 66 & 42 & 19 & $"$ \\
\hline 2 & 12 & & 44 & 52 & 66 & 45 & 19 & " \\
\hline 1 & 12 & & 44 & 59 & 66 & 50 & 19 & $"$ \\
\hline
\end{tabular}

Lat. Long, Date

No Returns

$\begin{array}{lllll}44 & 37 & 65 & 45 & 27 \\ & \text { Jan. } & 58\end{array}$ No Returns

$41 \quad 35.3 \quad 70 \quad 50 \quad 21$ Sept. '58

$\begin{array}{llllll}44 & 42 & 66 & 44 & 5 \mathrm{Feb} . & \end{array}$

$\begin{array}{lllll}44 & 52 & 65 & 26.5 & 1 \mathrm{Feb} .\end{array}$ No Returns

$\begin{array}{llllll}45 & 08 & 64 & 51 & 24 \mathrm{Jan} \cdot & 58\end{array}$

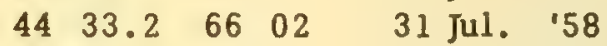

$\begin{array}{llllll}44 & 40.5 & 65 & 42.7 & 27 & \text { Feb. } 58\end{array}$

\begin{tabular}{lllll}
44 & 57 & 65 & 17 & $24 \mathrm{Jan}$. \\
\hline & 58
\end{tabular}

$\begin{array}{lllll}44 & 54.5 & 65 & 21 & 6 \mathrm{Mar} \text {. '58 }\end{array}$

$\begin{array}{llllll}44 & 18 & 66 & 19 & 20 \mathrm{Jan} . & 58\end{array}$

$\begin{array}{llllll}44 & 18 & 66 & 19 & 1 \text { Feb. '58 }\end{array}$

No Returns

$\begin{array}{llllll}44 & 15.3 & 66 & 20.3 & 25 \\ 4 a n . & \text { '58 }\end{array}$

$\begin{array}{llllll}44 & 11.5 & 66 & 10 & 4 \mathrm{Mar} . & \end{array}$

$\begin{array}{lllllll}37 & 56.6 & 75 & 19.2 & 12 \text { Oct. '59 }\end{array}$ No Returns 
Albatross III Cruise 107 (cont'd)

Released

Recovered

No.

Bottles

Sta.\# Released Bottle \# Lat. Long. Date

Lat. Long. Date

\begin{tabular}{|c|c|c|c|c|c|c|c|c|}
\hline 94 & 12 & & 44 & 31 & 67 & 11 & 19 & Jan. \\
\hline 93 & 12 & & 44 & 24 & 67 & 26 & 19 & $"$ \\
\hline 92 & 12 & & 44 & 00 & 67 & 37 & 19 & $"$ \\
\hline 91 & 12 & & 44 & 00 & 68 & 32 & 20 & " \\
\hline 90 & 12 & & 43 & 40 & 68 & 33 & 20 & " \\
\hline 89 & 12 & & 43 & 38 & 69 & 00 & 20 & " \\
\hline \multirow[t]{5}{*}{88} & 12 & $41826 \mathrm{~N}$ & 43 & 22 & 70 & 00 & 20 & \\
\hline & & $41830 \mathrm{~N}$ & 43 & 22 & 70 & 00 & 20 & $"$ \\
\hline & & $41832 N$ & 43 & 22 & 70 & 00 & 20 & 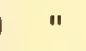 \\
\hline & & $41834 \mathrm{~N}$ & 43 & 22 & 70 & 00 & 20 &  \\
\hline & & $41836 \mathrm{~N}$ & 43 & 22 & 70 & 00 & 20 & \\
\hline
\end{tabular}

No Returns

"

"11

II

11

11

$\begin{array}{llllll}41 & 50.5 & 70 & 00.3 \quad \text { l Mar. '58 }\end{array}$

$\begin{array}{llllll}41 & 54 & 70 & 01 & 8 & \mathrm{Apr} . \\ 4\end{array}$

$\begin{array}{llllll}42 & 42.3 & 70 & 46.3 & 23 & \mathrm{Mar} . \\ 4 & 58\end{array}$

$\begin{array}{lllllll}41 & 53 & 70 & 00.6 & 22 \\ 4 a y & 58\end{array}$

$\begin{array}{lllllll}42 & 00 & 70 & 05 & \text { Spring } & \end{array}$ 
Delaware 57-9 III

\section{$28-29$ January}

1958

Odd numbers ballasted.

Released

No.

Bottles

Sta。\# Released Bottle \#

86

85

84

$83 \quad 12$

$82 \quad 12$

$81 \quad 12$
12

12

$41902 N$ $41904 \mathrm{~N}$ $41906 \mathrm{~N}$

12 $41839 \mathrm{~B}$ $41840 \mathrm{~N}$ $41841 \mathrm{~B}$ $41843 \mathrm{~B}$ $41848 \mathrm{~N}$

2

Lat.

Long.

\begin{tabular}{lllll}
42 & 39.5 & 69 & 31.5 & 28 \\
\hline$a n$
\end{tabular}

$41 \quad 56 \quad 70 \quad 26 \quad 28 \quad$ "

$4156 \quad 70 \quad 26 \quad 28 \quad$ "

$41 \quad 56 \quad 70 \quad 26 \quad 28 \quad "$

$\begin{array}{lllllll}42 & 20 & 70 & 38 & 28\end{array}$

$\begin{array}{lllllll}42 & 20 & 70 & 38 & 28\end{array}$

$\begin{array}{llllll}42 & 20 & 70 & 38 & 28\end{array}$

$42 \quad 20$

$\begin{array}{ll}70 & 38\end{array}$

28

28

$\begin{array}{ll}70 & 38\end{array}$

$42 \quad 20$

$\begin{array}{ll}70 & 00\end{array}$ $\begin{array}{llll}42 & 42 & 70 & 32 \\ 43 & 00 & 70 & 27\end{array}$ $\begin{array}{llll}42 & 42 & 70 & 32 \\ 43 & 00 & 70 & 27\end{array}$

\section{Recovered}

Lat. Long. Date

\section{No Returns}

$4150 \quad 70 \quad 00.2 \quad 3$ Feb. '58

$4150 \quad 70 \quad 00.2 \quad 2$ Mar. '58

$\begin{array}{llllll}4150 & 70 & 00.2 & 2 \mathrm{Mar} . & & \end{array}$

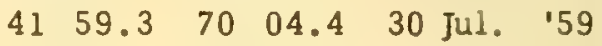

$\begin{array}{llllll}41 & 54 & 70 & 01.5 & 8 \text { Jun. '58 }\end{array}$

$\begin{array}{llllll}42 & 03.7 & 70 & 14.6 & 4 \mathrm{May} & 58\end{array}$

$41 \quad 59.5 \quad 70 \quad 04.5 \quad 4$ Mar. '58

$\begin{array}{llllll}41 & 48 & 70 & 00.7 & 9 & \text { Mar. '58 }\end{array}$

No Returns

" 
Sackville 37

$6-27$ February

1958

All bottles weighted.

$8 \mathrm{oz}$, beverage bottles.

$1 / 2$ gal. weighted bottles with metal drags.

Released

Recovered

No.

Bottles

Sta.\# Released Bottle \# Lat. Long。 Date

Lat. Long . Date

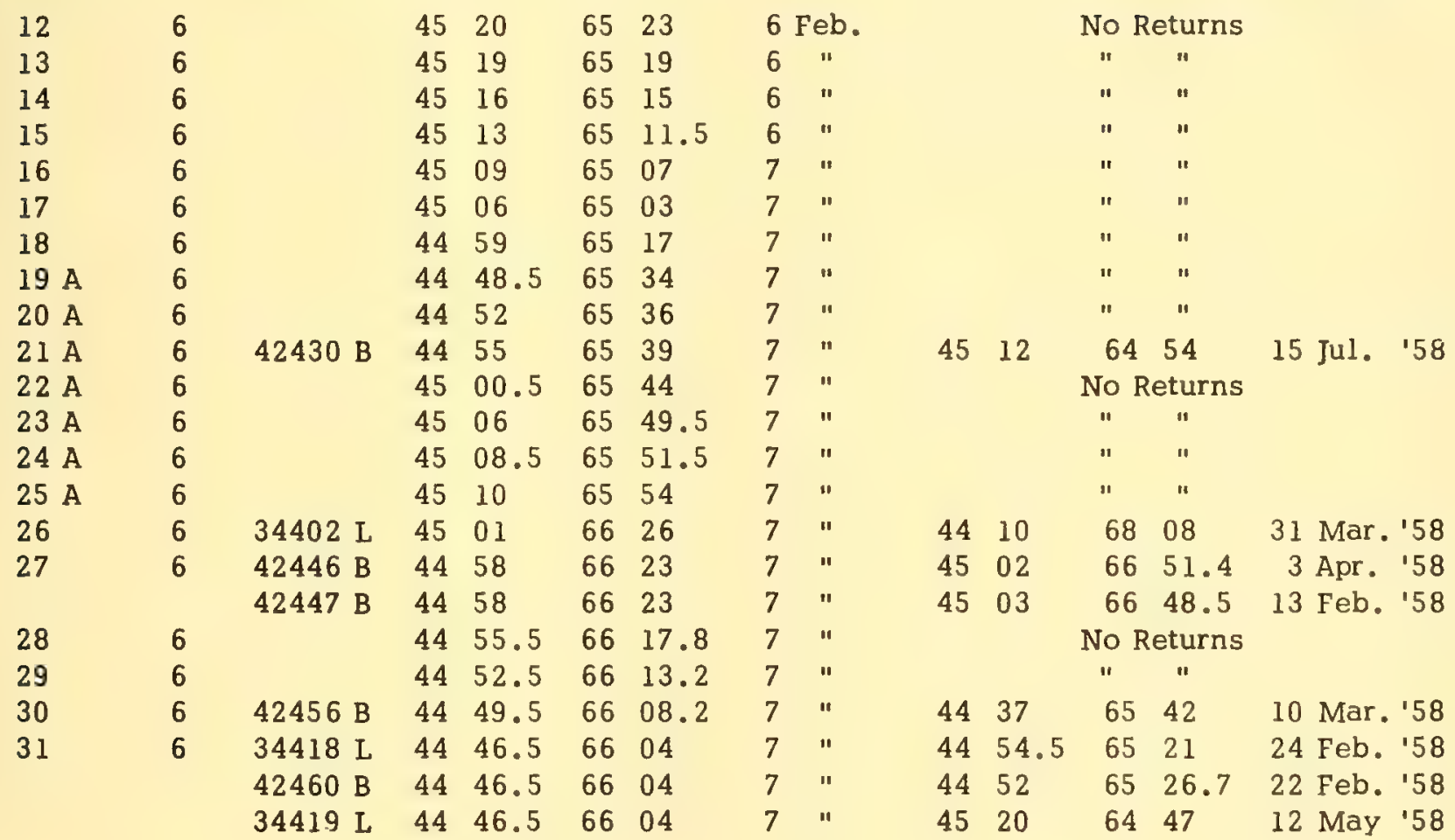


Sackville 37 (cont'd)

Released

Recovered

No.

Bottles

Sta.\# Released Bottle \# Lat. Long. Dat

\begin{tabular}{|c|c|c|c|c|c|c|c|c|c|c|c|c|c|c|}
\hline 11 & 6 & & 44 & 43.5 & 65 & 59 & & eb. & \multicolumn{6}{|c|}{ No Returns } \\
\hline 32 & 6 & $34425 \mathrm{~L}$ & 44 & 40.5 & 65 & 54 & 7 & $"$ & 44 & 56.6 & 65 & 17 & $15 \mathrm{Feb}$ & $' 58$ \\
\hline $10 \mathrm{~A}$ & 6 & & 44 & 25 & 66 & 14.2 & 8 & $"$ & \multicolumn{6}{|c|}{ No Returns } \\
\hline $9 \mathrm{~A}$ & 6 & & 44 & 28 & 66 & 17 & 8 & $"$ & & & $"$ & $"$ & & \\
\hline $8 \mathrm{~A}$ & 6 & $34434 \mathrm{~L}$ & 44 & 32 & 66 & 21 & 8 & $"$ & 42 & 02.6 & 70 & 10.6 & 27 Apr. & '58 \\
\hline $7 \mathrm{~A}$ & 6 & & 44 & 35 & 66 & 24 & 8 & $"$ & \multicolumn{6}{|c|}{ No Returns } \\
\hline $6 \mathrm{~A}$ & 6 & $34440 \mathrm{~L}$ & 44 & 39 & 66 & 28 & 8 & $"$ & 41 & 15 & 70 & 10 & 24 May & '58 \\
\hline $5 \mathrm{~A}$ & 6 & $42484 \mathrm{~B}$ & 44 & 42 & 66 & 31 & 8 & $"$ & 41 & 17.5 & 70 & 13 & 17 Jun. & '58 \\
\hline & & $34443 \mathrm{D}$ & 44 & 42 & 66 & 31 & 8 & $"$ & 44 & 39 & 65 & 51 & $24 \mathrm{Feb}$ & '58 \\
\hline $4 \mathrm{~A}$ & 6 & & 44 & 46 & 66 & 35 & 8 & $"$ & \multicolumn{6}{|c|}{ No Returns } \\
\hline $3 \mathrm{~A}$ & 6 & & 44 & 49.5 & 66 & 38.5 & 8 & $"$ & & & $"$ & $"$ & & \\
\hline $2 \mathrm{~A}$ & 6 & & 44 & 53 & 66 & 42 & 8 & $"$ & & & $"$ & $"$ & & \\
\hline $1 \mathrm{~A}$ & 5 & $42496 \mathrm{~B}$ & 44 & 59.5 & 66 & 49 & 8 & $"$ & 45 & 00.2 & 66 & 55.7 & 10 Mar. & $' 58$ \\
\hline 95 & 12 & & 44 & 57 & 66 & 51 & 9 & $"$ & \multicolumn{6}{|c|}{ No Returns } \\
\hline 94 & 12 & & 44 & 33 & 67 & 14 & 9 & $"$ & & & $"$ & $"$ & & \\
\hline $93 \mathrm{~A}$ & 12 & & 44 & 21 & 67 & 35 & 9 & $"$ & & & $"$ & $"$ & & \\
\hline 92 & 12 & & 44 & 00 & 67 & 37 & 9 & $"$ & & & $"$ & $"$ & & \\
\hline $91 \mathrm{~A}$ & 12 & & 43 & 56 & 68 & 33 & 10 & $n$ & & & $"$ & $"$ & & \\
\hline 90 & 12 & & 43 & 41 & 68 & 33 & 10 & $"$ & & & $"$ & " & & \\
\hline 89 & 12 & & 43 & 41 & 69 & 00 & 10 & $"$ & & & $"$ & $"$ & & \\
\hline 88 & 12 & & 43 & 21.5 & 69 & 00 & 10 & $"$ & & & $"$ & $"$ & & \\
\hline $87 \mathrm{~A}$ & 12 & & 43 & 20 & 70 & 12 & 10 & $"$ & & & $"$ & $"$ & & \\
\hline 86 & 12 & & 42 & 40 & 69 & 42 & 10 & $"$ & & & $"$ & $"$ & & \\
\hline $85 \mathrm{~A}$ & 12 & & 42 & 02 & 70 & 24 & 11 & $"$ & & & $"$ & $"$ & & \\
\hline $84 \mathrm{~A}$ & 12 & & 42 & 20 & 70 & 32 & 11 & $"$ & & & $"$ & $"$ & & \\
\hline 83 & 12 & & 42 & 41 & 70 & 00 & 11 & $"$ & & & $"$ & $"$ & & \\
\hline 80 & 12 & & 42 & 56 & 69 & 42 & 11 & ' & & & $"$ & $"$ & & \\
\hline 79 & 12 & & 42 & 52 & 69 & 00 & 11 & $"$ & & & $"$ & $"$ & & \\
\hline 78 & 12 & & 42 & 47 & 68 & 09 & 11 & " & & & $"$ & " & & \\
\hline 77 & 12 & & 42 & 35 & 66 & 00 & 12 & $"$ & & & " & " & & \\
\hline 76 & 12 & & 42 & 30 & 65 & 10.5 & 12 & $"$ & & & " & " & & \\
\hline A & 12 & & 42 & 38.5 & 65 & 00 & 12 & $"$ & & & $"$ & $"$ & & \\
\hline B & 12 & & 42 & 46.5 & 64 & 51 & 12 & $"$ & & & $"$ & " & & \\
\hline C & 12 & & 42 & 55 & 64 & 44 & 12 & " & & & " & " & & \\
\hline$D$ & 12 & & 43 & 03 & 64 & 37 & 12 & $"$ & & & $"$ & $"$ & & \\
\hline
\end{tabular}


No.

Bottles

Sta.\# Released

\begin{tabular}{|c|c|c|c|c|c|c|c|c|}
\hline$E$ & 12 & & 43 & 11 & 64 & 30 & 12 & Feb。 \\
\hline$F$ & 12 & & 43 & 18 & 64 & 25 & 12 & $n$ \\
\hline G & 12 & & 43 & 25 & 64 & 19 & 12 & $"$ \\
\hline $\mathrm{H}$ & 12 & 43567 B & 43 & 43 & 64 & 07 & 12 & $"$ \\
\hline I & 12 & & 43 & 57 & 63 & 51 & 12 & $"$ \\
\hline$J$ & 12 & & 44 & 04 & 63 & 45 & 12 & $"$ \\
\hline $\mathbf{K}$ & 12 & & 44 & 14 & 63 & 36 & 12 & " \\
\hline $\mathrm{L}$ & 12 & & 44 & 17.5 & 63 & 26 & 12 & $"$ \\
\hline \multirow[t]{2}{*}{$75 \mathrm{~A}$} & 12 & $42713 \mathrm{~B}$ & 43 & 15 & 66 & 05 & 16 & n \\
\hline & & $42723 \mathrm{~B}$ & 43 & 15 & 66 & 05 & 16 & $"$ \\
\hline 74 & 12 & 42734 B & 42 & 37.5 & 66 & 28 & 18 & " \\
\hline 73 & 12 & & 42 & 00 & 66 & 50.5 & 19 & $"$ \\
\hline 72 & 12 & & 41 & 30 & 67 & 07 & 19 & " \\
\hline 71 & 12 & & 41 & 00 & 67 & 26 & 19 & $"$ \\
\hline 70 & 12 & & 40 & 28 & 67 & 45 & 19 & $"$ \\
\hline 69 & 12 & & 40 & 00 & 68 & 00 & 19 & $"$ \\
\hline 68 & 12 & & 40 & 00 & 69 & 00 & 20 & $"$ \\
\hline 67 & 12 & & 40 & 00 & 70 & 00 & 20 & $"$ \\
\hline 66 & 12 & & 40 & 40 & 70 & 00 & 20 & $"$ \\
\hline 65 & 12 & & 40 & 40.5 & 69 & 00 & 20 & $n$ \\
\hline 64 & 12 & & 40 & 40.5 & 68 & 00 & 20 & " \\
\hline 63 & 12 & & 40 & 40.5 & 67 & 00 & 21 & $"$ \\
\hline 62 & 12 & & 41 & 15.5 & 66 & 13 & 21 & " \\
\hline 61 & 12 & & 41 & 15 & 67 & 00 & 21 & $"$ \\
\hline 60 & 12 & & 41 & 15.5 & 68 & 00 & 21 & $"$ \\
\hline 59 & 12 & & 41 & 15.5 & 69 & 00 & 22 & $"$ \\
\hline $58 \mathrm{~A}$ & 12 & & 41 & 15 & 69 & 16 & 22 & $"$ \\
\hline $57 \mathrm{~A}$ & 12 & 42935 & 41 & 37.5 & 69 & 39.5 & 22 & $"$ \\
\hline 56 & 12 & & 41 & 54 & 69 & 00 & 22 & $"$ \\
\hline 55 & 12 & & 41 & 53 & 68 & 00 & 22 & $n$ \\
\hline 54 & 12 & & 41 & 56 & 67 & 00 & 22 & $n$ \\
\hline 53 & 12 & & 41 & 49 & 65 & 37 & 23 & $"$ \\
\hline 52 & 12 & & 42 & 10 & 65 & 36 & 23 & $"$ \\
\hline 51 & 12 & & 42 & 03 & 66 & 00 & 23 & $"$ \\
\hline
\end{tabular}

Lat. Long. Date

No Returns

$\begin{array}{llllll}41 & 20 & 69 & 59.8 & 12 & \text { Aug. } \\ & 58\end{array}$ No Returns

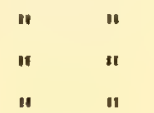

$\begin{array}{llllll}43 & 48 & 66 & 10 & 13 \\ \text { Mar. '58 }\end{array}$

$44 \quad 38.5 \quad 66 \quad 42.1 .30 \mathrm{Mar}$. '58

$\begin{array}{llllll}41 & 46.5 & 70 & 29.7 & 3 \mathrm{Apr} . & \end{array}$ No Returns

$\begin{array}{lllll}32 & 18.3 & 64 & 51.4 & 3 \text { Jun. '58 }\end{array}$ No Returns






\section{Released}

\section{Recovered}

No.

Bottles

\begin{tabular}{|c|c|c|c|c|c|c|c|c|}
\hline 50 & 12 & & 42 & 21.5 & 66 & 30 & 23 & Feb. \\
\hline 49 & 12 & & 42 & 04 & 67 & 00 & 23 & $"$ \\
\hline 48 & 12 & & 42 & 20 & 67 & 30 & 23 & $"$ \\
\hline 47 & 12 & & 41 & 58 & 68 & 00 & 23 & $"$ \\
\hline 46 & 12 & & 42 & 10.5 & 68 & 30 & 23 & $"$ \\
\hline 45 & 12 & & 42 & 11 & 69 & 30 & 24 & $"$ \\
\hline 44 & 12 & 43090 B & 42 & 30 & 69 & 30 & 25 & $"$ \\
\hline 43 & 12 & & 42 & 30 & 68 & 00 & 25 & $"$ \\
\hline 42 & 12 & $43114 \mathrm{~B}$ & 42 & 32 & 67 & 00 & 26 & $"$ \\
\hline 41 & 12 & & 42 & 40 & 67 & 00 & 26 & $"$ \\
\hline 40 & 12 & & 43 & 10.5 & 67 & 00 & 26 & $"$ \\
\hline \multirow[t]{2}{*}{39} & 12 & $43146 \mathrm{~B}$ & 43 & 22.5 & 68 & 00 & 26 & $"$ \\
\hline & & $43153 \mathrm{~B}$ & 43 & 22.5 & 68 & 00 & 26 & $"$ \\
\hline 38 & 12 & & 43 & 45 & 68 & 00 & 27 & $"$ \\
\hline \multirow[t]{2}{*}{37} & 12 & $43173 \mathrm{~B}$ & 43 & 40.5 & 67 & 00 & 27 & $"$ \\
\hline & & $43178 \mathrm{~B}$ & 43 & 40.5 & 67 & 00 & 27 & $"$ \\
\hline $36 \mathrm{~A}$ & 12 & & 43 & 44 & 66 & 31 & 27 & $"$ \\
\hline $35 \mathrm{~A}$ & 12 & $43194 \mathrm{~B}$ & 43 & 57 & 66 & 45 & 27 & $"$ \\
\hline $34 \mathrm{~A}$ & 12 & $43207 \mathrm{~B}$ & 44 & 08 & 66 & 31 & 27 & n \\
\hline $33 \mathrm{~A}$ & 12 & & 44 & 18 & 66 & 43 & 27 & " \\
\hline
\end{tabular}

Lat. Long. Date

\begin{tabular}{llllll}
40 & 50.8 & 72 & 27.5 & 5 & Mar. \\
\hline
\end{tabular}

No Returns

$\begin{array}{lllll}40 & 50.6 & 72 & 28.7 & 21 \text { Sept. '58 }\end{array}$ No Returns

$\begin{array}{llllll}41 & 44.9 & 70 & 25.7 & 6 \text { Dec. '58 }\end{array}$

$\begin{array}{llllll}41 & 56.7 & 70 & 36 & 27 & \text { Apr. } 58\end{array}$ No Returns

$\begin{array}{lllllll}41 & 20.4 & 70 & 43 & 27 & \mathrm{Jul} . & \text { '58 }\end{array}$

$\begin{array}{lllll}45 & 23.2 & 64 & 27.4 & 10 \\ \text { Sept. '58 }\end{array}$ No Returns

$\begin{array}{llllll}41 & 16 & 70 & 12.4 & 21 \mathrm{Jul} . & 159\end{array}$

$\begin{array}{lllll}41 & 53.3 & 70 & 00.9 & 21 \\ \text { Jun. } & 58\end{array}$ No Returns 
Sackville 38

4 - 5 April

1958

All bottles ballasted.

Released

No.

Bottles

Sta.\# Released Bottle \# Lat. Long. Date

$\begin{array}{llllllll}108 & 12 & 42 & 00 & 60 & 48 & 4 & \text { Apr . }\end{array}$

$109 \quad 12$

$110 \quad 12$

$111 \quad 12$

112

113

114

115

$\begin{array}{llllll}42 & 32 & 61 & 24 & 4 & \text { " }\end{array}$

$\begin{array}{llllllll}42 & 51 & 61 & 44 & 4\end{array}$

$\begin{array}{llllllll}43 & 10 & 62 & 03 & 4\end{array}$

$\begin{array}{llllll}43 & 28 & 62 & 22 & 4 & \end{array}$

$\begin{array}{llllllll}43 & 54 & 62 & 48 & 5 & \text { " }\end{array}$

$\begin{array}{lllllll}44 & 24 & 63 & 28 & 5 & \end{array}$

$\begin{array}{lllllll}44 & 24 & 63 & 28 & 5\end{array}$
$44 \quad 16 \quad 63 \quad 14 \quad 5$ "
Recovered

Lat. Long. Date

No Returns

"

11

MI

$\begin{array}{llllll}44 & 38.4 & 63 & 19 & 31 \text { May '58 }\end{array}$

$\begin{array}{lllll}44 & 27.3 & 63 & 44.7 & 21 \text { Jun. '58 }\end{array}$ No Returns

$\begin{array}{lllll}43 & 49 & 64 & 50 & 15 \\ 4 a y & & \end{array}$

$\begin{array}{lllll}43 & 49 & 64 & 50 & 4\end{array}$ Jun. '58 
Metacomet 58-1

18 - 26 April

1958

All bottles ballasted.

Released

Recovered

No.

Bottles

Sta.\# Released Bottle \# Lat. Long. Date Lat. Long. Date

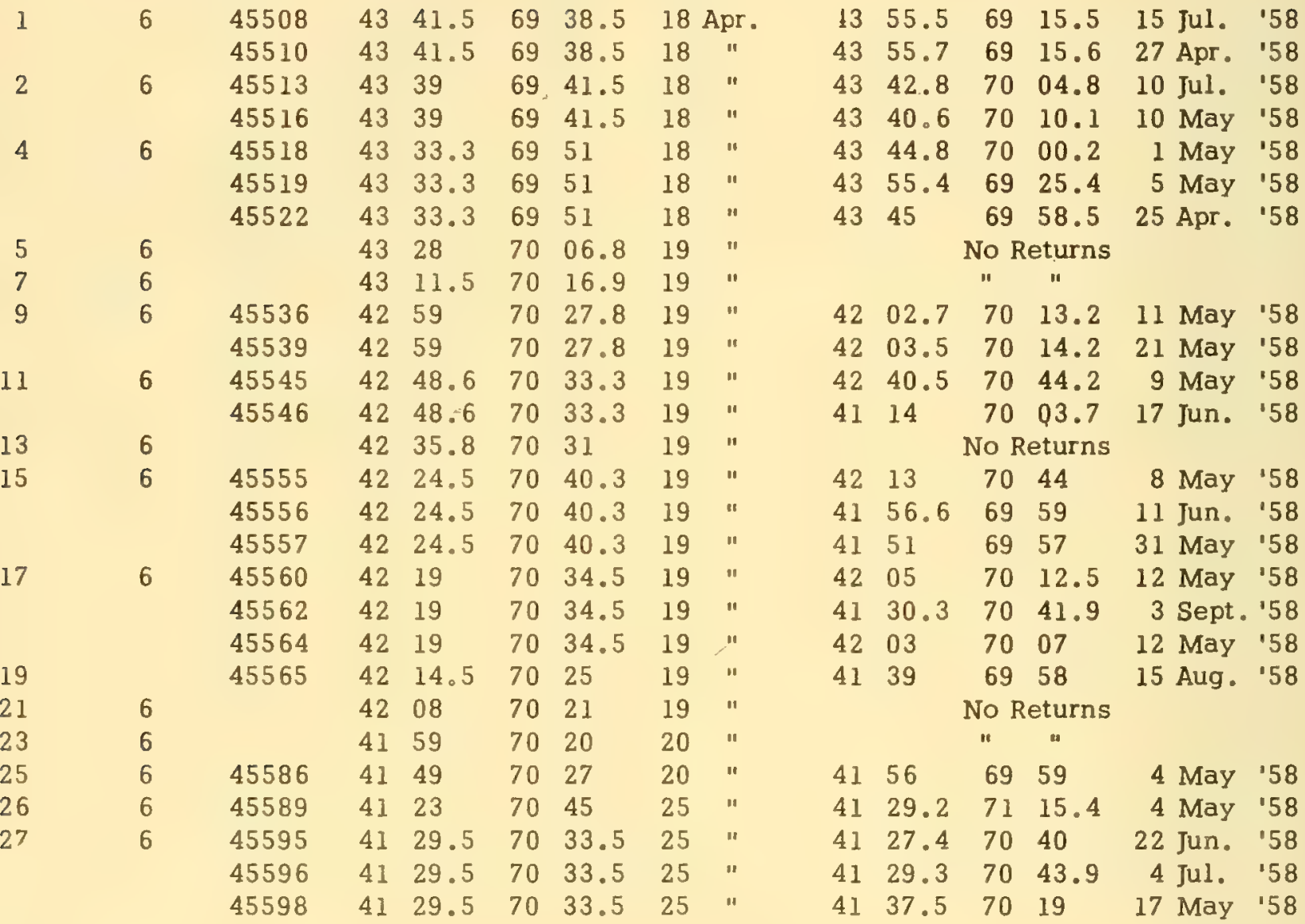


Metacomet 58- 1 (cont'd)

Released

Recovered

No.

Bottles

Sta.\# Released Bottle \# Lat. Long. Date Lat. Long. Date

\begin{tabular}{|c|c|c|c|c|c|c|c|c|c|c|c|c|c|c|}
\hline \multirow[t]{3}{*}{29} & 6 & 45603 & 41 & 28 & 70 & 13.5 & 25 & Apr. & 41 & 39 & 69 & 58 & 28 Jun. & $' 58$ \\
\hline & & 45604 & 41 & 28 & 70 & 13.5 & 25 & $"$ & 41 & 36.3 & 70 & 25.8 & 29 Jun. & $' 58$ \\
\hline & & 45606 & 41 & 28 & 70 & 13.5 & 25 & $"$ & 41 & 36.4 & 70 & 23.9 & 7 May & $' 58$ \\
\hline \multirow[t]{2}{*}{31} & 6 & 45610 & 41 & 26 & 70 & 01 & 25 & $"$ & 41 & 16.5 & 69 & 57.6 & 15 May & '58 \\
\hline & & 45612 & 41 & 26 & 70 & 01 & 25 & $n$ & 41 & 31.7 & 70 & 57.3 & 14 Jun. & .58 \\
\hline 33 & 6 & & 41 & 26 & 69 & 49 & 25 & $"$ & \multicolumn{6}{|c|}{ No Returns } \\
\hline \multirow[t]{2}{*}{35} & 6 & 45621 & 41 & 28 & 69 & 44 & 25 & $"$ & 41 & 23.5 & 70 & 30.3 & 7 Sept. & . 58 \\
\hline & & 45622 & 41 & 28 & 69 & 44 & 25 & $"$ & 41 & 30 & 71 & 02.7 & 26 May & 158 \\
\hline \multirow[t]{3}{*}{37} & $i$ & 45626 & 41 & 31 & 69 & 45.5 & 25 & $"$ & 41 & 37 & 70 & 52 & 25 Aug. & '58 \\
\hline & & 45628 & 41 & 31 & 69 & 45.5 & 25 & $"$ & 41 & 38.3 & 70 & 38.6 & 30 Jun. & 58 \\
\hline & & 45630 & 41 & 31 & 69 & 45.5 & 25 & $"$ & 41 & 23.4 & 70 & 30.6 & 28 May & 58 \\
\hline \multirow[t]{3}{*}{39} & 6 & 45632 & 41 & 40.5 & 69 & 47 & 25 & " & 41 & 15 & 70 & 09 & 2 Jun. & 8 \\
\hline & & 45633 & 41 & 40.5 & 69 & 47 & 25 & $"$ & 41 & 32.7 & 70 & 34.7 & 6 Jun. & 58 \\
\hline & & 45636 & 41 & 40.5 & 69 & 47 & 25 & $"$ & 41 & 19.2 & 69 & 59.3 & 26 Jun. & 58 \\
\hline \multirow[t]{3}{*}{41} & 6 & 45637 & 41 & 52 & 69 & 50.5 & 25 & $"$ & 41 & 22.6 & 70 & 30.4 & 27 May & 8 \\
\hline & & 45638 & 41 & 52 & 69 & 50.5 & 25 & $"$ & 41 & 23 & 70 & 23 & $19 \mathrm{Jul}$. & 58 \\
\hline & & 45639 & 41 & 52 & 69 & 50.5 & 25 & $"$ & 41 & 28 & 70 & 55 & $13 \mathrm{Jul}$ & 58 \\
\hline \multirow[t]{3}{*}{42} & 12 & 45644 & 42 & 06.5 & 69 & 58 & 26 & $"$ & 42 & 04 & 70 & 07 & 8 May & 58 \\
\hline & & 45648 & 42 & 06.5 & 69 & 58 & 26 & $"$ & 41 & 57 & 69 & 57 & 3 May & 58 \\
\hline & & 45652 & 42 & 06.5 & 69 & 58 & 26 & $"$ & 41 & 57 & 69 & 59.2 & 24 May & ' 58 \\
\hline \multirow[t]{5}{*}{43} & 12 & 45655 & 42 & 13.5 & 70 & 08 & 26 & $"$ & 41 & 17.4 & 70 & 05.5 & 21 Jun. & 58 \\
\hline & & 45660 & 42 & 13.5 & 70 & 08 & 26 & $"$ & 41 & 22.8 & 70 & 02.5 & 17 Jun. & 58 \\
\hline & & 45661 & 42 & 13.5 & 70 & 08 & 26 & $"$ & 41 & 22.6 & 70 & 41.4 & 4 Aug. & '58 \\
\hline & & 45662 & 42 & 13.5 & 70 & 08 & 26 & $"$ & 41 & 30.5 & 71 & 04 & $8 \mathrm{Jul}$. & 58 \\
\hline & & 45664 & 42 & 13.5 & 70 & 08 & 26 & $"$ & 41 & 39.8 & 70 & 39 & $23 \mathrm{Jul}$ & 58 \\
\hline \multirow[t]{8}{*}{44} & 12 & 45668 & 42 & 22 & 70 & 21 & 26 & $"$ & 41 & 45.5 & 70 & 07.4 & 24 Apr. & ' 59 \\
\hline & & 45670 & 42 & 22 & 70 & 21 & 26 & $"$ & 42 & 01.5 & 70 & 11.5 & 14 Jun. & 58 \\
\hline & & 45672 & 42 & 22 & 70 & 21 & 26 & $"$ & 41 & 58.5 & 70 & 40.8 & 2 May & '58 \\
\hline & & 45673 & 42 & 22 & 70 & 21 & 26 & $"$ & 41 & 50 & 69 & 56.5 & 24 Aug. & 158 \\
\hline & & 45.674 & 42 & 22 & 70 & 21 & 26 & $"$ & 41 & 45.6 & 70 & 08 & 17 May & '58 \\
\hline & & 45676 & 42 & 22 & 70 & 21 & 26 & $"$ & 42 & 02 & 70 & 05.7 & 17 May & 58 \\
\hline & & 45677 & 42 & 22 & 70 & 21 & 26 & $"$ & 41 & 18.5 & 70 & 47.6 & 17 Aug. & '58 \\
\hline & & 45678 & 42 & 22 & 70 & 21 & 26 & $"$ & 42 & 02 & 70 & 13 & $11 \mathrm{May}$ & \\
\hline
\end{tabular}


Metacomet 58-1 (cont'd)

Released

Recovered

No.

Bottles

Sta.\# Released Bottle \# Lat. Long. Date Lat. Long. Date

45

$1245681 \quad 42 \quad 31 \quad 70 \quad 35 \quad 26$ Apr.

$\begin{array}{llllll}42 & 27 & 70 & 56 & 29 & \mathrm{Apr} .158\end{array}$

$\begin{array}{llllllll}45685 & 42 & 31 & 70 & 35 & 26 & \end{array}$

$\begin{array}{lllll}42 & 27 & 70 & 56 & 29 \\ \mathrm{Apr} . & \text { '58 }\end{array}$

$\begin{array}{llllllll}45688 & 42 & 31 & 70 & 35 & 26 & \end{array}$

$\begin{array}{llllll}42 & 27 & 70 & 56.3 & 28 \text { Apr. '58 }\end{array}$

45690

4231

$\begin{array}{ll}70 & 35\end{array}$

$26 "$

$\begin{array}{llll}42 & 27 & 70 & 56\end{array}$

29 Apr. 58

$42 \quad 27$

29 May '58 
6 - 7 May

1958

All bottles ballasted.

Released

Recovered

No.

Bottles

Sta.\# Released Bottle\# Lat. Long. Date

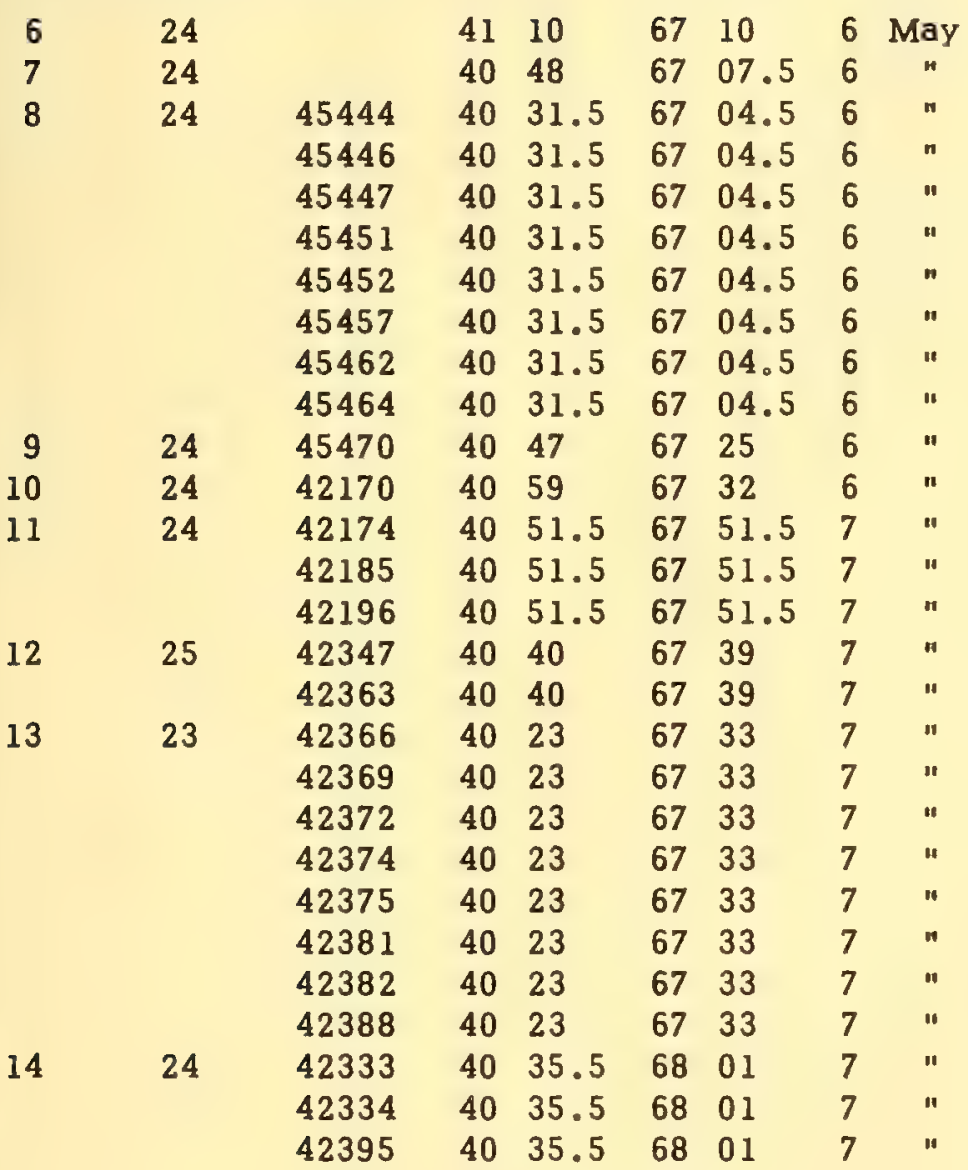

Lat. Long. Date

No Returns

$\begin{array}{llllll}41 & 19.2 & 71 & 48.5 & 5 \text { Aug. } 58\end{array}$

$\begin{array}{llllll}41 & 19 & 70 & 47.6 & 6 \mathrm{Jul} & \end{array}$

$\begin{array}{llllll}41 & 42 & 70 & 40 & 14 \mathrm{Jul} . & \end{array}$

$\begin{array}{llllll}41 & 29.2 & 71 & 15.4 & 3 \text { Nov. '58 }\end{array}$

$\begin{array}{lllll}40 & 38 & 73 & 12 & 1 \text {. Sept. '58 }\end{array}$

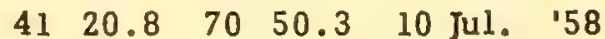

$\begin{array}{lllll}41 & 21.3 & 70 & 29.9 & \text { 17. Sept. "58 }\end{array}$

$\begin{array}{llllll}41 & 22.5 & 71 & 28.9 & 27 & \text { Jul. }\end{array}$

$\begin{array}{llllll}41 & 20.8 & 70 & 40.8 & 3 \text { Aug. "58 }\end{array}$

$\begin{array}{llllll}41 & 27 & 70 & 48.3 & 18 \text { Aug. '58 }\end{array}$

$\begin{array}{llllll}41 & 41 & 70 & 08 & 9 & \mathrm{Apr} \text {. '59 }\end{array}$

$\begin{array}{lllllll}41 & 30.5 & 71 & 04 & 17 & \mathrm{Jul} . & 58\end{array}$

$\begin{array}{lllllll}41 & 26.4 & 71 & 28.3 & 7 & \text { Aug. '58 }\end{array}$

$\begin{array}{llllll}41 & 22.5 & 71 & 33 & 7 \text { Aug. '58 }\end{array}$

$\begin{array}{lllll}41 & 39.5 & 69 & 59 & 15\end{array}$ Aug. '58

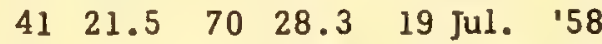

$\begin{array}{llllll}41 & 38 & 70 & 38.6 & 10 & \text { Sept. '58 }\end{array}$

$\begin{array}{llllll}41 & 30.3 & 71 & 03.5 & 12 & \text { Sept. '58 }\end{array}$

$\begin{array}{llllll}41 & 37.5 & 70 & 17 & 13 & \text { Aug. '58 }\end{array}$

$\begin{array}{llllll}41 & 37.6 & 70 & 15 & 29 \mathrm{Jul} . & 58\end{array}$

$\begin{array}{lllllll}41 & 30.5 & 71 & 04.5 & 12 & \mathrm{Jul} . & ' 58\end{array}$

$\begin{array}{lllllll}41 & 20.5 & 70 & 42.5 & 27 & \text { Jul. } & 58\end{array}$



$\begin{array}{llllll}41 & 28.2 & 70 & 38 & 21 \mathrm{Jul} . & \text { '58 }\end{array}$

$\begin{array}{llllll}41 & 14.8 & 70 & 05 & 20 & \mathrm{Jul} .\end{array}$

$\begin{array}{llllll}41 & 20.9 & 70 & 33 & 8 \text { Aug. '58 }\end{array}$ 
Released

Recovered

No.

Bottles

Sta.\# Released Bottle \# Lat. Long. Date Long. Date

\begin{tabular}{|c|c|c|c|c|c|c|c|c|c|c|c|c|}
\hline 24 & 42294 & 40 & 43 & 68 & 20 & 7 & May & 41 & 19 & 71 & 49 & I Aug. \\
\hline & 42298 & 40 & 43 & 68 & 20 & 7 & $"$ & 41 & 32.3 & 69 & 49.6 & $13 \mathrm{Jul}$. \\
\hline & 42301 & 40 & 43 & 68 & 20 & 7 & $"$ & 41 & 37.9 & 70 & 19.4 & $30 \mathrm{Jul}$. \\
\hline & 42304 & 40 & 43 & 68 & 20 & 7 & " & 41 & 20 & 70 & 49.5 & 8 Aug. \\
\hline & 42306 & 40 & 43 & 68 & 20 & 7 & $"$ & 41 & 39.8 & 70 & 05.3 & $21 \mathrm{Jul}$. \\
\hline & 42315 & 40 & 43 & 68 & 20 & 7 & $"$ & 41 & 31.8 & 70 & 40.5 & 17 Jul. \\
\hline 24 & 41910 & 40 & 25.5 & 68 & 16 & 7 & $"$ & 41 & 22.2 & 70 & 50 & $5 \mathrm{Jul}$. \\
\hline & 41914 & 40 & 25.5 & 68 & 16 & 7 & $"$ & 41 & 23.4 & 70 & 30.2 & 15 Aug. \\
\hline & 41920 & 40 & 25.5 & 68 & 16 & 7 & $"$ & 41 & 27 & 70 & 48 & 24 Aug. \\
\hline & 42321 & 40 & 25.5 & 68 & 16 & 7 & $"$ & 41 & 18.5 & 70 & 05 & 24 Aug. \\
\hline 23 & 41924 & 40 & 31 & 68 & 40.5 & 7 & $"$ & 41 & 30.5 & 71 & 04 & 13 Aug. \\
\hline & 41928 & 40 & 31 & 68 & 40.5 & 7 & $"$ & 41 & 29.2 & 71 & 15.2 & $31 \mathrm{Jul}$. \\
\hline & 41936 & 40 & 31 & 68 & 40.5 & 7 & $"$ & 41 & 19 & 70 & 47.6 & $6 \mathrm{Jul}$. \\
\hline & 41935 & 40 & 31 & 68 & 40.5 & 7 & " & 40 & 35 & 69 & 15 & $25 \mathrm{Apr}$. \\
\hline
\end{tabular}




\section{Metacomet 58-2}

8 - 11 May

1958

Surface type bottles, odd numbers ballasted.

Released

Recovered

No.

Bottles

Sta.\# Released Bottle \# Lat. Long. Date Lat. Long. Date



$\begin{array}{llllllllllllllll}18 & 8 & 45706 & \mathrm{~N} & 43 & 42 & 69 & 56 & 10 & \prime & 45 & 22 & 64 & 23.5 & 12 & \text { May }\end{array}$

$\begin{array}{llllllll}19 & 8 & 43 & 42 & 69 & 59 & 10 & \end{array}$

No Returns

$\begin{array}{lllllllllll}21 & 8 & 45718 & \mathrm{~N} & 42 & 14 & 70 & 20 & 11 & \text { " }\end{array}$

$\begin{array}{lllllll}41 & 38.5 & 69 & 57.8 & 27 & \text { Jul. '58 }\end{array}$

$\begin{array}{llllllll}45722 & \mathrm{~N} & 42 & 14 & 70 & 20 & 11\end{array}$

$\begin{array}{lllllll}41 & 21 & 70 & 33 & 17 & \mathrm{Jul} . & 158\end{array}$

22 $\begin{array}{llllllll}45730 & N & 42 & 21 & 70 & 18 & 11\end{array}$

$\begin{array}{llllll}41 & 20.8 & 70 & 50.4 & 14 & \text { Jun. '58 }\end{array}$ No Returns

$\begin{array}{llllll}42 & 35 & 70 & 13 & 11 & \end{array}$

" $"$

24

$\begin{array}{lllllllll}8 & & & 42 & 40 & 70 & 09 & 11 & " \\ 8 & 45759 & \text { B } & 42 & 45 & 70 & 06 & 11 & \end{array}$

"

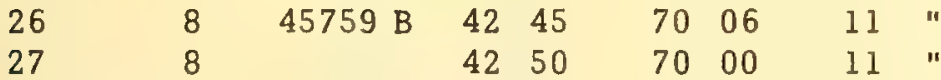

$\begin{array}{llllll}44 & 39.1 & 66 & 43.6 \quad 8 \mathrm{Nov} . & & \end{array}$

$\begin{array}{llllllll}8 & 45778 & \mathrm{~N} & 42 & 57 & 69 & 58 & 11\end{array}$

$43 \quad 04$

$69 \quad 57 \quad 11 \quad "$ No Returns

$\begin{array}{llllll}58 & 54 & 03 & 10 & 13 & \text { Aug. '59 }\end{array}$ No Returns 
Crawford 18

17 May - 3 June

1958

All 8 oz. ballasted bottles.

Released

Recovered

No.

Bottles

Sta.\# Released Bottle \# Lat. Long. Date Lat. Long. Date

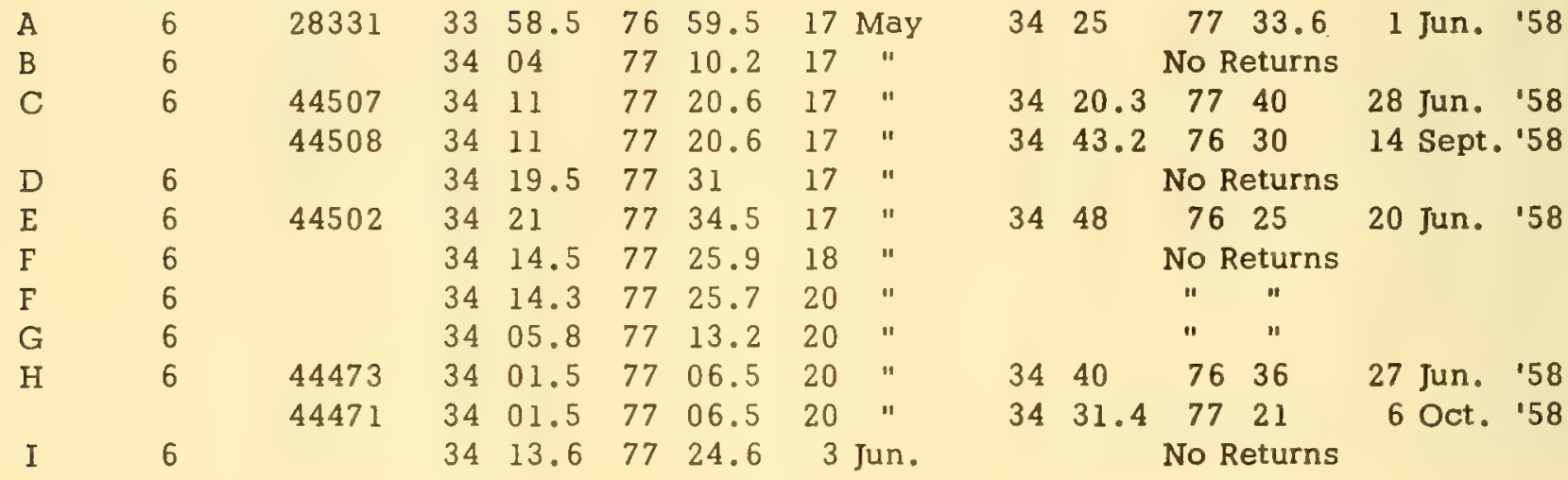


Albatross III Cruise 111

$20-27$ May

1958

Odd numbers ballasted.

Released

Recovered

No.

Bottles

Sta.\# Released Bottle \# Lat. Long. Date Lat. Long. Date

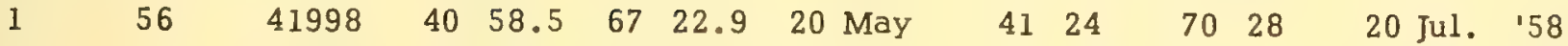

$\begin{array}{lllllllllllllllll}2 & 56 & 42002 & 41 & 01 & 67 & 15 & 21 & \| & 41 & 42 & 67 & 45 & 4 & \text { Jul. } & 58\end{array}$

$\begin{array}{llllllllllll}42003 & 41 & 01 & 67 & 15 & 21 & \text { " } & 35 & 13.8 & 75 & 31.6 & 23 \\ \text { Oct. '58 }\end{array}$

$\begin{array}{llllllllllll}42014 & 41 & 01 & 67 & 15 & 21 & \prime & 41 & 30.5 & 71 & 05 & 13 . \text { Aug. '58 }\end{array}$

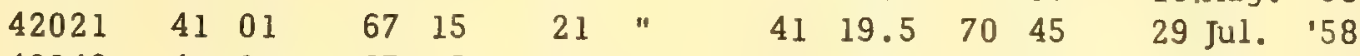

$\begin{array}{lllllllllllll}42043 & 41 & 01 & 67 & 15 & 21 & \text { " } & 41 & 39.5 & 69 & 59 & 15 & \text { Aug. '58 }\end{array}$

$\begin{array}{llllllllllll}42054 & 41 & 01 & 67 & 15 & 21 & \text { " } & 41 & 39 & 70 & 10 & 30 \text { Aug. '58 }\end{array}$

$\begin{array}{llllllllllllllll}3 & 56 & 42057 & 41 & 03 & 67 & 16.8 & 21 & \text { " } & 41 & 38 & 70 & 38 & 20 & \text { Aug. '58 }\end{array}$

$\begin{array}{lllllllllllll}42060 & 41 & 03 & 67 & 16.8 & 21 & \prime & 41 & 16.3 & 70 & 13.2 & 12 & \text { Jul. } 58\end{array}$

$\begin{array}{lllllllllllllllll}42066 & 41 & 03 & 67 & 16.8 & 21 & \text { " } & 41 & 20 & 70 & 34 & 18 \text { Aug. '58 }\end{array}$

$\begin{array}{llllllllllllll}42096 & 41 & 03 & 67 & 16.8 & 21 & 1 & 41 & 21.5 & 71 & 37.5 & 4 \text { Aug. '58 }\end{array}$

$\begin{array}{lllllllllllll}45106 & 41 & 03 & 67 & 16.8 & 21 & 41 & 25 & 70 & 56.7 & 17 & \text { Sept. '58 }\end{array}$

\begin{tabular}{llllllllllllll}
4 & 47 & & 41 & 36.2 & 68 & 33.8 & 23 & \multicolumn{4}{c}{ No Returns } \\
5 & 48 & 42259 & 41 & 39.6 & 68 & 38 & 24 & $\prime \prime$ & 43 & 39 & 6507 & 20 & Oct. '58
\end{tabular}

$\begin{array}{lllllllllllllllll}6 & 48 & 42198 & 40 & 21.4 & 68 & 53.2 & 26 & \| & 41 & 39.2 & 70 & 07.5 & 27 & \text { Jul. } & 58\end{array}$

$\begin{array}{llllllllllll}42199 & 40 & 21.4 & 68 & 53.2 & 26 & " & 41 & 19 & 71 & 49.5 & 6 \text { Aug. '58 }\end{array}$

\begin{tabular}{llllllllllll}
42206 & 40 & 21.4 & 68 & 53.2 & 26 & 4 & 40 & 23 & 70 & 30 & 21 \\
\hline
\end{tabular}

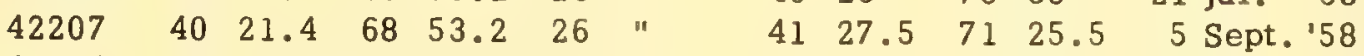

$\begin{array}{llllllllllll}42214 & 40 & 21.4 & 68 & 53.2 & 26 & \text { " } & 41 & 21.7 & 70 & 28.5 & 9\end{array}$ Jul. '58

$\begin{array}{llllllllllll}42229 & 40 & 21.4 & 68 & 53.2 & 26 & \| & 41 & 17.6 & 70 & 06.1 & 2 \\ \text { Jul. } 58\end{array}$

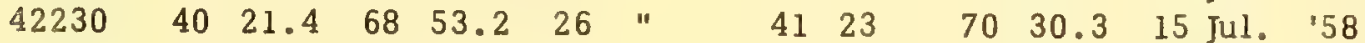

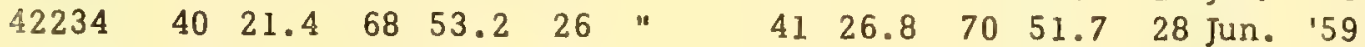



$\begin{array}{lllllllllllll}42241 & 40 & 21.4 & 68 & 53.2 & 26 & \prime & 41 & 49.2 & 71 & 51 & 30 & \text { Jul. '58 }\end{array}$

$\begin{array}{lllllllllllll}42244 & 40 & 21.4 & 68 & 53.2 & 26 & \text { " } & 41 & 23 & 71 & 28.6 & 3 \text { Aug. '58 }\end{array}$ 
No.

Bottles

Sta.\# Released Bottle \# Lat. Long. Date Lat. Long. Date

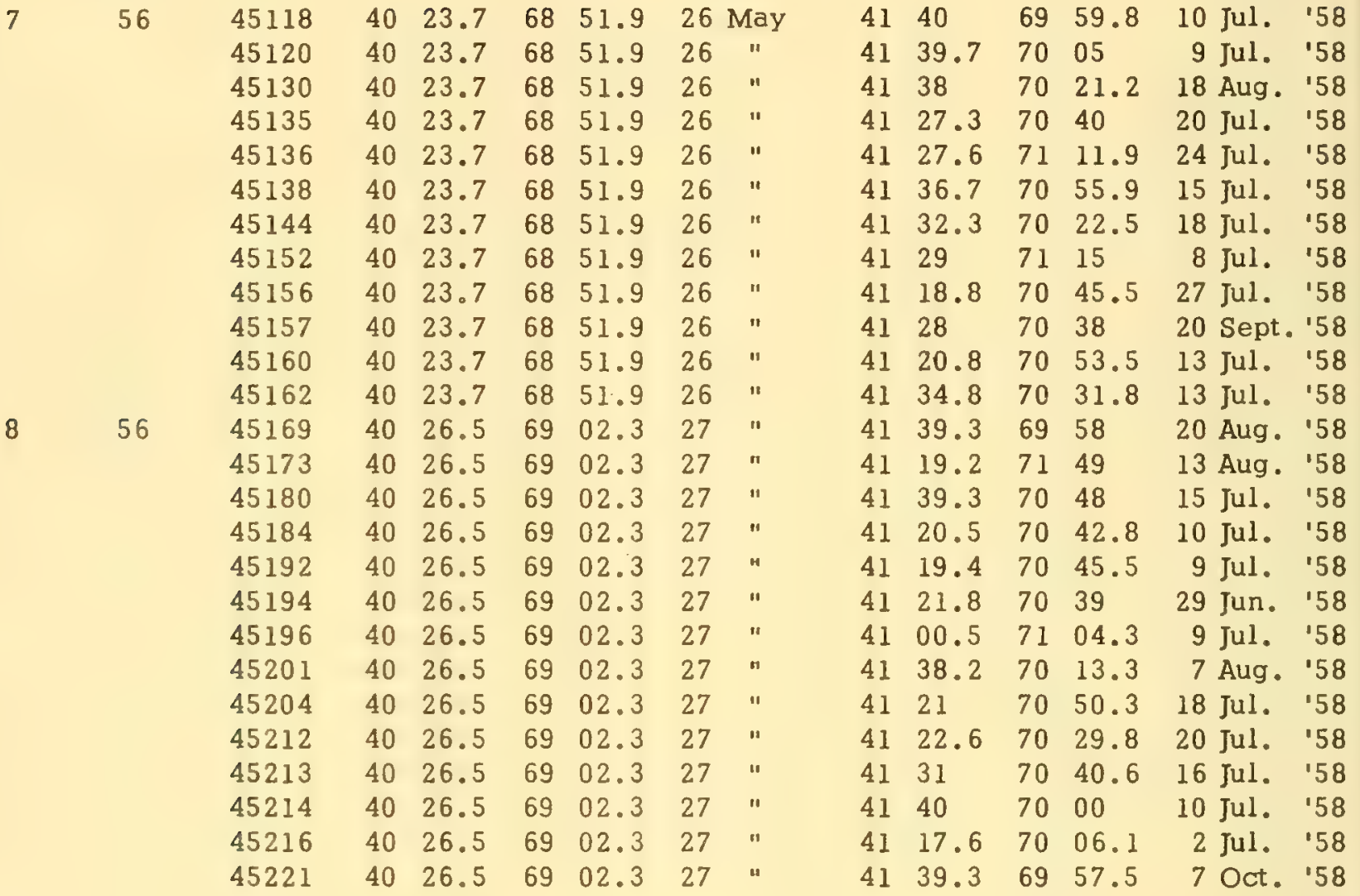


$\underline{\text { Sackville }} 39$

22 - 27 May

1958

Ballast and drag bottles.

Released

Recovered

No.

Bottles

Sta.\# Released Bottle \# Lat. Long. Date Lat. Long. Date

\begin{tabular}{|c|c|c|c|c|c|c|c|c|c|c|c|c|c|c|}
\hline \multirow[t]{2}{*}{12} & \multirow[t]{2}{*}{6} & $34696 \mathrm{~L}$ & 45 & 22 & 65 & 21 & 22 & May & 45 & 20 & 64 & 46.5 & 16 Jun. & 58 \\
\hline & & $51802 \mathrm{~B}$ & 45 & 22 & 65 & 21 & 22 & и & 45 & 23.5 & 64 & 27 & 24 Aug. & 158 \\
\hline 3 & 6 & $34701 \mathrm{D}$ & 45 & 19 & 65 & 19 & 22 & $"$ & 45 & 20 & 64 & 46.5 & 15 Jun. & 158 \\
\hline 4 & 6 & $34704 \mathrm{~L}$ & 45 & 16 & 65 & 15 & 22 & " & 45 & 26 & 64 & 53.5 & 6 Aug. & 158 \\
\hline 5 & 6 & $51810 \mathrm{~B}$ & 45 & 12.5 & 65 & 10.5 & 22 & $"$ & 44 & 54.4 & 66 & 21 & 2 Nov. & '58 \\
\hline \multirow[t]{2}{*}{16} & \multirow[t]{2}{*}{6} & $34710 \mathrm{D}$ & 45 & 07.5 & 65 & 07 & 22 & $"$ & 45 & 24 & 64 & 31.4 & 20 Jun. & - \\
\hline & & $34713 \mathrm{D}$ & 45 & 07.5 & 65 & 07 & 22 & $"$ & 45 & 10.4 & 64 & 46 & $14 \mathrm{Jul}$. & 58 \\
\hline \multirow[t]{2}{*}{7} & \multirow[t]{2}{*}{6} & $34711 \mathrm{~B}$ & 45 & 05 & 65 & 03 & 22 & $"$ & 45 & 10.3 & 64 & 46.8 & $14 \mathrm{Jul}$. & 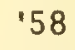 \\
\hline & & $34712 \mathrm{~L}$ & 45 & 05 & 65 & 03 & 22 & $"$ & 45 & 17 & 64 & 46.5 & 8 Jun. & \\
\hline \multirow[t]{3}{*}{18} & \multirow[t]{3}{*}{6} & $51819 \mathrm{~B}$ & 44 & 59.3 & 65 & 17.5 & 22 & $"$ & 45 & 04 & 66 & 44 & $25 \mathrm{Jul}$. & \\
\hline & & $34714 \mathrm{D}$ & 44 & 59.3 & 65 & 17.5 & 22 & $"$ & 44 & 57 & 65 & 54 & 2 Aug. & \\
\hline & & $34716 \mathrm{~L}$ & 44 & 59.3 & 65 & 17.5 & 22 & $"$ & 45 & 01.4 & 66 & 57.3 & 25 Nov. & 8 \\
\hline A & 6 & & 44 & 49.5 & 65 & 33.5 & 22 & $"$ & \multicolumn{6}{|c|}{ No Returns } \\
\hline$A$ & 6 & $34720 \mathrm{D}$ & 44 & 52.5 & 65 & 36.5 & 22 & $"$ & 44 & 54.5 & 65 & 24 & 23 May & \\
\hline \multirow[t]{4}{*}{$1 \mathrm{~A}$} & \multirow[t]{4}{*}{6} & $34723 \mathrm{D}$ & 44 & 55 & 65 & 39 & 22 & $"$ & 45 & 20 & 64 & 46.5 & 14 Jun. & 58 \\
\hline & & $34724 \mathrm{D}$ & 44 & 55 & 65 & 39 & 22 & $"$ & 44 & 57 & 65 & 17 & 24 May & 58 \\
\hline & & $34725 \mathrm{D}$ & 44 & 55 & 65 & 39 & 22 & $"$ & 44 & 55 & 65 & 19 & 24 May & \\
\hline & & $51830 \mathrm{~B}$ & 44 & 55 & 65 & 39 & 22 & $"$ & 45 & 14 & 64 & 03.5 & 25 Oct. & 58 \\
\hline & 6 & $34727 \mathrm{D}$ & 45 & 00.5 & 65 & 44 & 22 & $"$ & 45 & 05.1 & 66 & 39.2 & 14 Jun. & 58 \\
\hline & 6 & & 45 & 06.5 & 65 & 49 & 22 & $"$ & \multicolumn{6}{|c|}{ No Returns } \\
\hline & 6 & $34733 \mathrm{D}$ & 45 & 08.5 & 65 & 52 & 22 & $"$ & 45 & 05.7 & 66 & 38 & 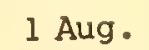 & \\
\hline & 6 & & 45 & 10 & 65 & 54 & 22 & $"$ & \multicolumn{6}{|c|}{ No Returns } \\
\hline & 6 & $34739 \mathrm{D}$ & 45 & 01.5 & 66 & 26.5 & 22 & $"$ & 45 & 06 & 66 & 29 & 28 May & 58 \\
\hline A & 6 & $51847 \mathrm{~B}$ & 44 & 58.5 & 66 & 22.5 & 22 & $"$ & 44 & 32 & 65 & 52.1 & 7 Jul. & 58 \\
\hline \multirow[t]{2}{*}{$8 \mathrm{~A}$} & \multirow[t]{2}{*}{6} & $34746 \mathrm{D}$ & 44 & 55.5 & 66 & 18 & 22 & $"$ & 44 & 50 & 66 & 59 & 6 Jun. & \\
\hline & & $51850 \mathrm{~B}$ & 44 & 55.5 & 66 & 18 & 22 & $"$ & 44 & 48.5 & 66 & 58.7 & 1 Jun. & 58 \\
\hline \multirow[t]{3}{*}{$9 \AA$} & \multirow[t]{3}{*}{6} & $34747 \mathrm{~L}$ & 44 & 52.5 & 66 & 13 & 22 & $"$ & 44 & 58 & 66 & 43.4 & 14 Jun. & 58 \\
\hline & & $34749 \mathrm{I}$ & 44 & 52.5 & 66 & 13 & 22 & $"$ & 45 & 29 & 65 & 10 & 28 Aug. & 8 \\
\hline & & $51854 \mathrm{~B}$ & 44 & 52.5 & 66 & 13 & 22 & $"$ & 45 & 10.3 & 64 & 46.8 & $14 \mathrm{Jul}$. & \\
\hline
\end{tabular}


Released

Recovered

No.

Bottles

Sta.\# Released Bottle \# Lat. Long. Date Lat. Long. Date

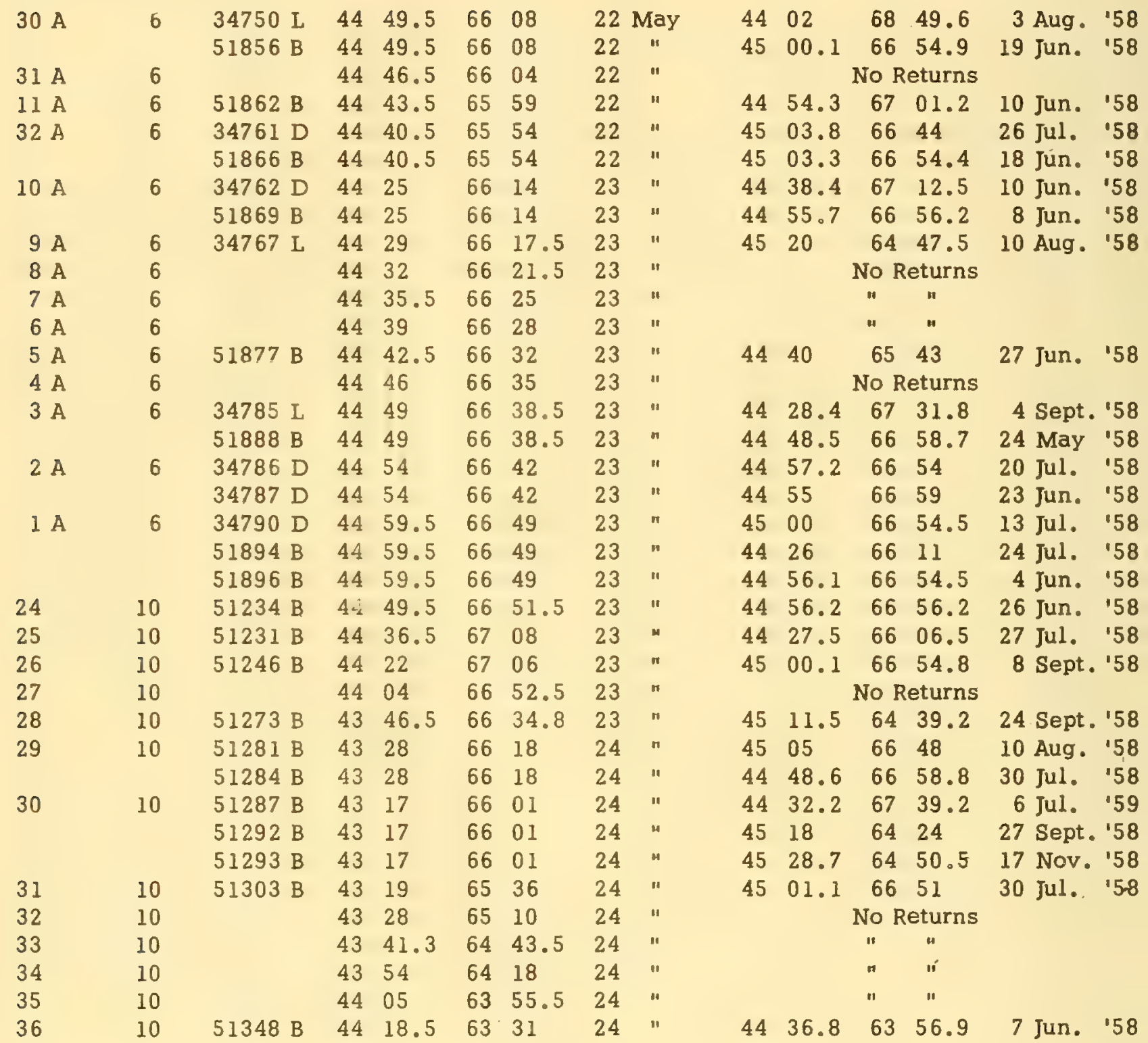


Sackville 39 (cont'd)

Released

Recovered

No.

Bottles

Sta.\# Released Bottle \# Lat. Long. Date Lat. Long. Date

\begin{tabular}{|c|c|c|c|c|c|c|c|c|c|c|c|c|c|c|}
\hline \multirow[t]{8}{*}{37} & 10 & $51356 \mathrm{~B}$ & 44 & 26 & 63 & 24 & 24 & May & 44 & 35.6 & 63 & 55.5 & 17 Jun. & 158 \\
\hline & & $51357 \mathrm{~B}$ & 44 & 26 & 63 & 24 & 24 & $"$ & 44 & 31.6 & 63 & 56.7 & 27 Sept. & 158 \\
\hline & & $51358 \mathrm{~B}$ & 44 & 26 & 63 & 24 & 24 & $"$ & 44 & 40 & 62 & 52 & 18 Jun. & 158 \\
\hline & & $51359 \mathrm{~B}$ & 44 & 26 & 63 & 24 & 24 & " & 44 & 34 & 63 & 55.7 & 2 Jul. & 158 \\
\hline & & $51360 \mathrm{~B}$ & 44 & 26 & 63 & 24 & 24 & $"$ & 44 & 36 & 63 & 29 & $30 \mathrm{May}$ & '58 \\
\hline & & $51361 \mathrm{~B}$ & 44 & 26 & 63 & 24 & 24 & $"$ & 44 & 30 & 63 & 52 & 1 Jul. & '58 \\
\hline & & $51362 \mathrm{~B}$ & 44 & 26 & 63 & 24 & 24 & $"$ & 44 & 39.5 & 63 & 33.5 & 2 Jun. & 158 \\
\hline & & $51363 \mathrm{~B}$ & 44 & 26 & 63 & 24 & 24 & $"$ & 44 & 38 & 63 & 56 & 13 Jun. & 158 \\
\hline \multirow[t]{2}{*}{39} & 10 & $51366 \mathrm{~B}$ & 44 & 15 & 63 & 17 & 24 & $"$ & 44 & 37.5 & 63 & 56 & 30 Jun. & 58 \\
\hline & & $51370 \mathrm{~B}$ & 44 & 15 & 63 & 17 & 24 & $"$ & 44 & 35 & 63 & 27.1 & $31 \mathrm{MaY}$ & '58 \\
\hline 41 & 10 & & 43 & 53.5 & 62 & 54 & 24 & $"$ & \multicolumn{6}{|c|}{ No Returns } \\
\hline 43 & 10 & & 43 & 30 & 62 & 27.5 & 25 & $"$ & & & $"$ & $"$ & & \\
\hline 45 & 10 & & 43 & 12 & 62 & 07 & 25 & $"$ & & & $"$ & $"$ & & \\
\hline 47 & 10 & & 42 & 51 & 61 & 45 & 25 & $"$ & & & $"$ & " & & \\
\hline 49 & 10 & $51419 \mathrm{~B}$ & 42 & 31 & 61 & 23 & 25 & $"$ & 63 & 50 & 09 & $40 \mathrm{E}$ & 4 Oct. & 159 \\
\hline 61 & 10 & & 41 & 55.5 & 62 & 58 & 26 & $"$ & \multicolumn{6}{|c|}{ No Returns } \\
\hline 62 & 10 & & 42 & 07.5 & 63 & 28 & 26 & $"$ & & & $"$ & $"$ & & \\
\hline 63 & 10 & & 42 & 17 & 63 & 49 & 26 & $"$ & & & $"$ & $"$ & & \\
\hline 64 & 10 & & 42 & 27 & 64 & 12 & 26 & $"$ & & & " & n & & \\
\hline 65 & 10 & & 42 & 35 & 64 & 37 & 26 & $n$ & & & $"$ & $"$ & & \\
\hline 66 & 10 & & 42 & 44 & 65 & 00 & 26 & $"$ & & & $"$ & $"$ & & \\
\hline 67 & 10 & & 42 & 56 & 65 & 23 & 26 & $"$ & & & $"$ & " & & \\
\hline 68 & 10 & & 42 & 51 & 65 & 46 & 26 & $"$ & & & $"$ & $"$ & & \\
\hline \multirow[t]{3}{*}{69} & 10 & $51506 \mathrm{~B}$ & 43 & 08 & 65 & 55 & 26 & $"$ & 44 & 22 & 66 & 05.6 & 16 Apr. & '59 \\
\hline & & $51507 \mathrm{~B}$ & 43 & 08 & 65 & 55 & 26 & $"$ & 44 & 26.8 & 66 & 01 & $6 \mathrm{Jul}$. & $' 58$ \\
\hline & & $51513 \mathrm{~B}$ & 43 & 08 & 65 & 55 & 26 & " & 44 & 26.3 & 66 & 01 & $30 \mathrm{Jul}$. & '58 \\
\hline 70 & 10 & & 43 & 28 & 66 & 16 & 26 & $"$ & \multicolumn{6}{|c|}{ No Returns } \\
\hline 71 & 10 & $51525 \mathrm{~B}$ & 43 & 47.5 & 66 & 32 & 26 & $"$ & 45 & 20 & 64 & 48 & 15 Sept. & 158 \\
\hline 72 & 10 & $51537 \mathrm{~B}$ & 44 & 09 & 66 & 33.5 & 26 & $"$ & 44 & 33 & 65 & 52 & 11 Aug. & '58 \\
\hline 73 & 10 & & 44 & 30 & 66 & 33.5 & 27 & $"$ & \multicolumn{5}{|c|}{ No Returns } & \\
\hline
\end{tabular}


Albatross III Cruise 117

9 - 15 September

1958

All $8 \mathrm{oz}$. bottles, ballasted.

Released

Recovered

No.

Bottles

Sta.\# Released Bottle \# Lat. Long. Date

\begin{tabular}{|c|c|c|c|c|c|c|c|}
\hline 12 & & 41 & 21.8 & 69 & 31 & 9 & Sept \\
\hline 12 & & 41 & 06 & 68 & 51 & 9 & $"$ \\
\hline 12 & & 40 & 49.4 & 68 & 14.1 & 10 & $"$ \\
\hline 12 & & 40 & 37.2 & 67 & 41 & 10 & $"$ \\
\hline 12 & & 40 & 31.5 & 67 & 04.5 & 10 & $"$ \\
\hline 12 & & 40 & 43 & 66 & 47.2 & 10 & $"$ \\
\hline 12 & & 40 & 55.2 & 66 & 30 & 10 & $"$ \\
\hline 12 & & 41 & 11 & 66 & 07.7 & 11 & " \\
\hline 12 & & 41 & 25.7 & 65 & 58.3 & 11 & $"$ \\
\hline 12 & & 41 & 39 & 65 & 58 & 12 & $"$ \\
\hline 12 & & 41 & 57 & 65 & 50 & 12 & $"$ \\
\hline 12 & 55817 & 42 & 14.3 & 65 & 53 & 12 & $"$ \\
\hline & 55821 & 42 & 14.3 & 65 & 53 & 12 & $"$ \\
\hline 12 & & 42 & 34 & 65 & 55 & 12 & $"$ \\
\hline 12 & 55845 & 42 & 53 & 65 & 52 & 12 & $"$ \\
\hline 12 & & 43 & 02 & 65 & 51 & 12 & $"$ \\
\hline 12 & 55867 & 42 & 37.3 & 66 & 10 & 12 & $"$ \\
\hline 12 & & 42 & 32 & 66 & 15 & 12 & $"$ \\
\hline 12 & & 42 & 18.6 & 66 & 24 & 12 & $"$ \\
\hline 12 & & 41 & 59.6 & 66 & 37.6 & 12 & $"$ \\
\hline 12 & & 41 & 32 & 66 & 56 & 13 & $"$ \\
\hline 12 & & 41 & 05 & 67 & 15 & 13 & " \\
\hline 12 & & 40 & 37.4 & 67 & 34.3 & 13 & $"$ \\
\hline 12 & & 40 & 20 & 67 & 46 & 13 & " \\
\hline 12 & & 40 & 00 & 68 & 00 & 13 & " \\
\hline 12 & & 40 & 24.2 & 68 & 13.7 & 13 & $"$ \\
\hline 12 & & 40 & 53.8 & 68 & 30.2 & 13 & " \\
\hline 12 & & 41 & 18 & 68 & 46.5 & 14 & $"$ \\
\hline 12 & & 41 & 43.4 & 69 & 06.8 & 14 & $"$ \\
\hline 12 & & 42 & 06.8 & 69 & 12.2 & 14 & $"$ \\
\hline 12 & 56035 & 42 & 07.0 & 69 & 50 & 14 & $"$ \\
\hline 12 & & 42 & 20.1 & 69 & 34 & 15 & " \\
\hline
\end{tabular}

Lat. Long. Date

No Returns

" "

"

"

II 11

II II

11

11

11 II

11 I1

$\begin{array}{llllll}43 & 59 & 66 & 09.2 & 23 & \text { Oct. '58 }\end{array}$

$\begin{array}{lllll}4411 & 11 & 10.5 & 1 \text { Nov. '58 }\end{array}$ No Returns

$\begin{array}{llllll}44 & 37.2 & 66 & 42.5 & 21 & \text { Oct. '58 }\end{array}$ No Returns

$\begin{array}{llllll}43 & 26.5 & 65 & 38 & \text { I Feb. '59 }\end{array}$

No Returns

$\begin{array}{lllllll}41 & 43.5 & 69 & 55.7 & 10 & \text { Oct. '58 }\end{array}$ No Returns 


\section{Dartmouth Cruise 1}

9 - 15 September

1958

All 8 oz. bottles, ballasted.

Released

Recovered

No.

Bottles

Sta.\# Released Bottle \# Lat. Long. Date Lat. Long. Date

\begin{tabular}{|c|c|c|c|c|c|c|c|c|}
\hline 12 & 41 & 26 & 69 & 30 & & ept. & No & turns \\
\hline 12 & 41 & 15.5 & 68 & 26 & 10 & $"$ & " & $"$ \\
\hline 12 & 41 & 02 & 67 & 18 & 10 & " & " & " \\
\hline 12 & 41 & 01.5 & 66 & 50 & 10 & " & $"$ & $"$ \\
\hline 12 & 41 & 21 & 66 & 27 & 10 & $"$ & " & $"$ \\
\hline 12 & 41 & 23 & 66 & 30 & 10 & " & " & " \\
\hline 12 & 41 & 23 & 66 & 23.5 & 11 & $"$ & n & $"$ \\
\hline 12 & 41 & 27 & 66 & 20.5 & 11 & $"$ & $"$ & $"$ \\
\hline 12 & 41 & 26 & 66 & 28 & 11 & $"$ & " & $"$ \\
\hline 12 & 41 & 37 & 66 & 17.5 & 12 & $"$ & $"$ & $"$ \\
\hline 12 & 41 & 51 & 66 & 22 & 12 & $"$ & " & " \\
\hline 12 & 41 & 52.5 & 66 & 23 & 12 & $"$ & $"$ & " \\
\hline 12 & 41 & 39 & 66 & 18.5 & 12 & $"$ & " & $"$ \\
\hline 12 & 41 & 47.5 & 66 & 17.5 & 13 & $"$ & $"$ & $"$ \\
\hline 12 & 41 & 50.5 & 66 & 21 & 13 & $"$ & $"$ & $"$ \\
\hline 12 & 41 & 52 & 66 & 20 & 13 & $"$ & $"$ & $"$ \\
\hline 12 & 41 & 51 & 66 & 21.5 & 13 & $"$ & $"$ & $"$ \\
\hline 12 & 41 & 51 & 66 & 20.5 & 14 & " & " & " \\
\hline 12 & 42 & 05.5 & 66 & 30 & 14 & $"$ & " & $"$ \\
\hline 12 & 42 & 04.5 & 67 & 07 & 14 & $"$ & $"$ & $"$ \\
\hline 12 & 41 & 12 & 68 & 44 & 15 & $"$ & $"$ & $"$ \\
\hline 12 & 41 & 09 & 68 & 41 & 15 & $"$ & $"$ & $"$ \\
\hline 12 & 41 & 05.5 & 68 & 46 & 15 & $"$ & " & $"$ \\
\hline 12 & 41 & 04.5 & 68 & 46 & 15 & $"$ & $"$ & $"$ \\
\hline 12 & 41 & 16 & 69 & 04 & 15 & $"$ & $"$ & $"$ \\
\hline
\end{tabular}


$\underline{\text { Albatross III Cruise } 118}$

23 September - 1 October

1958

Odd numbers ballasted.

Released

Recovered

No.

Bottles

Sta.\# Released Bottle \# Lat. Long. Date Lat. Long. Date

\begin{tabular}{|c|c|c|c|c|c|c|c|c|}
\hline 2 & 12 & & 43 & 47.3 & 68 & 32.8 & 23 & Sept \\
\hline 3 & 12 & 56618 & 43 & 52.1 & 67 & 57.9 & 23 & $"$ \\
\hline $3 \mathrm{~A}$ & 12 & 56631 & 43 & 52.7 & 67 & 34.8 & 23 & " \\
\hline 4 & 12 & & 43 & 53.9 & 67 & 16.4 & 23 & " \\
\hline 5 & 12 & & 43 & 54.5 & 66 & 32.3 & 23 & $"$ \\
\hline 6 & 12 & & 43 & 38.6 & 66 & 19.3 & 24 & $"$ \\
\hline 7 & 12 & & 43 & 33.7 & 66 & 52.8 & 24 & $"$ \\
\hline 8 & 12 & & 43 & 39.1 & 67 & 34 & 24 & " \\
\hline 9 & 12 & 56700 & 43 & 34.9 & 68 & 12.6 & 24 & $"$ \\
\hline & 12 & & 43 & 36.4 & 68 & 51 & 24 & " \\
\hline & 12 & & 43 & 36.2 & 69 & 34.8 & 24 & " \\
\hline & 12 & & 43 & 13 & 70 & 10.3 & 25 & $\mu$ \\
\hline 3 & 12 & & 43 & 17.3 & 69 & 55.8 & 25 & " \\
\hline 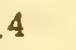 & 12 & & 43 & 18.8 & 69 & 19.7 & 25 & $"$ \\
\hline & 12 & & 43 & 15 & 68 & 35.8 & 25 & $"$ \\
\hline 0 & 12 & & 43 & 16 & 67 & 58 & 25 & " \\
\hline & 12 & & 43 & 19 & 67 & 19 & 25 & $"$ \\
\hline 3 & 12 & 56816 & 43 & 10.5 & 66 & 34 & 26 & " \\
\hline & 12 & 56819 & 43 & 12.9 & 66 & 02.5 & 26 & ." \\
\hline & 12 & & 43 & 11.4 & 65 & 16.7 & 26 & " \\
\hline I & 12 & & 43 & 29.9 & 64 & 56.3 & 26 & " \\
\hline & 12 & & 43 & 16 & 64 & 38 & 26 & 18 \\
\hline & 12 & & 42 & 53 & 64 & 53.3 & 27 & $"$ \\
\hline & 12 & & 42 & 54.9 & 65 & 33.3 & 27 & " \\
\hline 8 & 12 & & 42 & 58.4 & 66 & 32.8 & 27 & $" 1$ \\
\hline $4 \mathrm{~A}$ & 12 & & 42 & 51.5 & 70 & 36 & 27 & " \\
\hline 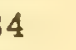 & 12 & & 42 & 52 & 70 & 15.6 & 29 & $"$ \\
\hline & 12 & & 42 & 53.5 & 69 & 38.2 & 29 & " \\
\hline & 12 & & 42 & 58.2 & 68 & 53 & 30 & $"$ \\
\hline
\end{tabular}

No Returns

$\begin{array}{lllllll}44 & 41.3 & 65 & 45.5 & 22 & \text { Oct. '58 }\end{array}$

$\begin{array}{lllllll}44 & 41.2 & 65 & 47.5 & 22 & \text { Oct. '58 }\end{array}$

No Returns

$\begin{array}{lllllll}44 & 15.5 & 66 & 08 & 20 & \text { Oct. '58 }\end{array}$ No Returns

$\begin{array}{llllll}44 & 20 & 66 & 07.5 & 24 \mathrm{Feb} . & \end{array}$

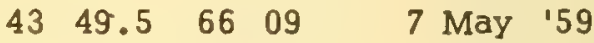

No Returns

$\begin{array}{lllll}44 & 15.7 & 66 & 08 & 3 \text { Nov。 "58 }\end{array}$ No Returns






\section{Albatross III Cruive 118 (cont'd)}

Released

Recovered

No.

Bottles

Sta.\# Released Bottle \# Lat. Long. Date Lat. Long. Date

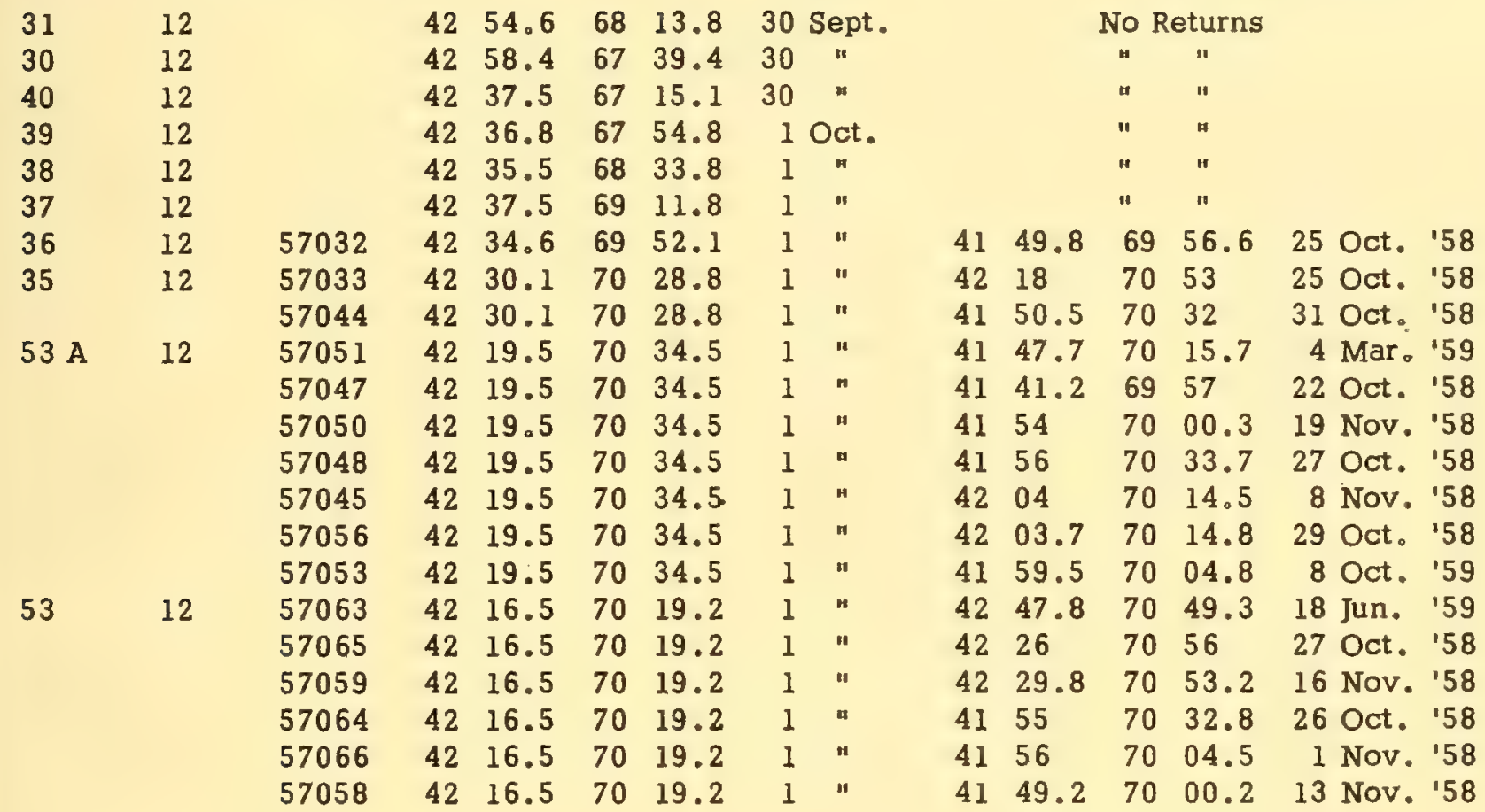


Delaware 58-5

6 - 14 October

1958

All bottles ballasted.

Released

Recovered

No.

Bottles

St.1.\# Released Bottle \# Lat. Long. Date Lat. Long. Date

\begin{tabular}{|c|c|c|c|c|c|c|c|c|c|c|c|c|c|}
\hline 44 & & 42 & 29 & 69 & 31 & & Det. & \multicolumn{6}{|c|}{ No Returns } \\
\hline 43 & & 42 & 30 & 67 & 57 & 7 & $"$ & & & $"$ & $"$ & & \\
\hline 42 & & 42 & 30 & 67 & 02 & 7 & $"$ & & & " & . & & \\
\hline 74 & & 42 & 38 & 66 & 30 & 7 & $n$ & & & $n$ & $"$ & & \\
\hline 77 & & 42 & 35 & 66 & 00 & 7 & $"$ & & & 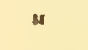 & $"$ & & \\
\hline 76 & & 42 & 30 & 65 & 10 & 7 & $\omega$ & & & $n$ & $"$ & & \\
\hline 75 & 45984 & 43 & 24 & 66 & 03 & 8 & $"$ & 43 & 52.5 & 66 & 09.8 & 18 Oct. & '58 \\
\hline & 45987 & 43 & 24 & 66 & 03 & 8 & $"$ & 44 & 33 & 66 & 02 & 2 Nov. & '58 \\
\hline & 45991 & 43 & 24 & 66 & 03 & 8 & $"$ & 43 & 59.5 & 66 & 09.2 & 16 Nov. & $' 58$ \\
\hline 73 & & 42 & 00 & 66 & 50 & 8 & $"$ & \multicolumn{6}{|c|}{ No Returns } \\
\hline 12 & & 41 & 29 & 67 & 10 & 8 & $"$ & & & $"$ & $"$ & & \\
\hline 71 & & 40 & 59 & 67 & 26 & 8 & $"$ & & & $"$ & n & & \\
\hline 12 & & 40 & 29 & 67 & 44 & 9 & $"$ & & & $"$ & " & & \\
\hline 12 & & 40 & 02 & 68 & 01 & 9 & $"$ & & & " & $"$ & & \\
\hline 12 & & 40 & 01 & 68 & 59 & 9 & $"$ & & & " & " & & \\
\hline 12 & & 39 & 59 & 69 & 52 & 9 & " & & & $"$ & $"$ & & \\
\hline 12 & & 40 & 37 & 70 & 00 & 9 & $"$ & & & " & $"$ & & \\
\hline 12 & & 40 & 41 & 69 & 05 & 10 & " & & & $"$ & $"$ & & \\
\hline 12 & & 40 & 40 & 68 & 01 & 10 & $\mu$ & & & $"$ & $"$ & & \\
\hline 12 & & 40 & 42 & 67 & 01 & 10 & $"$ & & & $"$ & $"$ & & \\
\hline 12 & & 41 & 16 & 67 & 01 & 10 & $"$ & & & $"$ & $"$ & & \\
\hline 12 & & 41 & 15 & 68 & 00 & 11 & $"$ & & & $"$ & $"$ & & \\
\hline 12 & & 41 & 16.5 & 68 & 57 & 11 & $"$ & & & $n$ & n & & \\
\hline 12 & & 41 & 14 & 69 & 18 & II & $"$ & & & " & $"$ & & \\
\hline 12 & 46184 & 41 & 49 & 69 & 00 & 12 & $"$ & 41 & 50.3 & 69 & 56.6 & 23 Oct. & $\cdot 58$ \\
\hline & 46180 & 41 & 49 & 69 & 00 & 12 & $"$ & 41 & 20 & 70 & 02.2 & 3 May & 159 \\
\hline
\end{tabular}


Released

Recovered

No.

Bottles

Sta.\# Released Bottle \# Lat. Long. Date Lat. Iong. Date

\begin{tabular}{|c|c|c|c|c|c|c|c|c|}
\hline 55 & 12 & 41 & 50.5 & 68 & 00.5 & 12 Oct. & No & Returns \\
\hline 54 & 12 & 41 & 50 & 67 & 06 & 12 & $"$ & " \\
\hline 53 & 12 & 41 & 48 & 65 & 37 & 13 & " & $"$ \\
\hline 52 & 12 & 42 & 09.5 & 65 & 37.5 & 13 & $"$ & $"$ \\
\hline 51 & 12 & 42 & 07 & 66 & 02 & 13 & $"$ & n \\
\hline 50 & 12 & 42 & 22 & 66 & 31.5 & 13 & $"$ & " \\
\hline 49 & 12 & 42 & 07.5 & 66 & 58 & 13 & " & $"$ \\
\hline 48 & 12 & 42 & 20 & 67 & 24.5 & 13 & $"$ & $"$ \\
\hline 47 & 12 & 41 & 58.5 & 68 & 00 & 14 & $n$ & " \\
\hline 46 & 12 & 42 & 09.5 & 68 & 29 & 14 & $"$ & " \\
\hline 45 & 12 & 42 & 10.5 & 69 & 29 & 14 & " & " \\
\hline
\end{tabular}


Albatross III Cruise 119

$8-16$ October

1958

Odd numbers ballasted.

Released

Recovered

No.

Bottles

Sta.\# Released Bottle\# Lat. Long. Date Lat. Long. Date

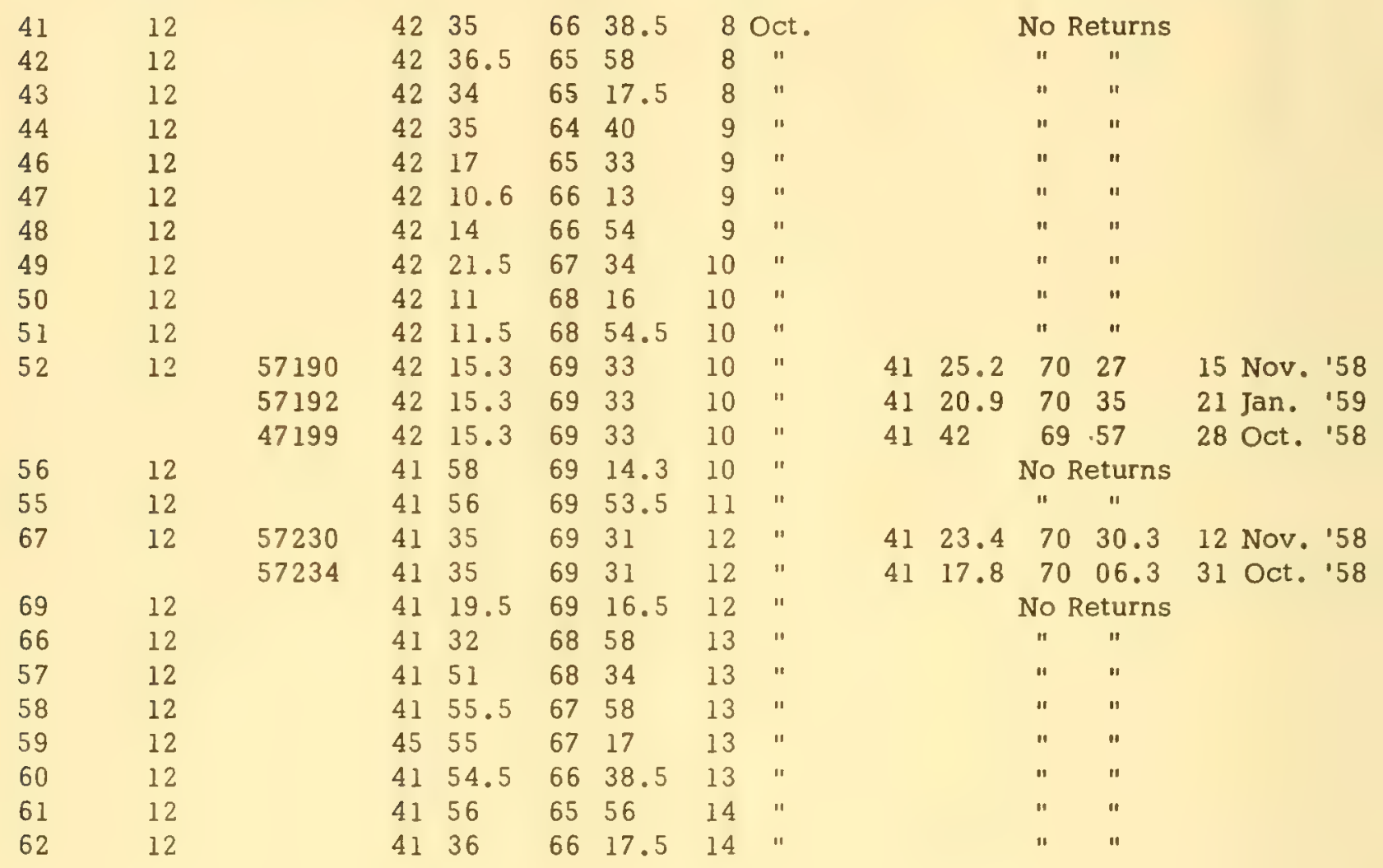




$$
\text { Released }
$$

Recovered

No.

Bottles

Sta.\# Released

$\begin{array}{llllllll}63 & 12 & 41 & 40.5 & 66 & 56 & 14 & \text { Oct. } \\ 64 & 12 & 41 & 37.5 & 67 & 37.5 & 14 & " \\ 65 & 12 & 41 & 40 & 68 & 12.5 & 14 & " \\ 70 & 12 & 41 & 18.5 & 68 & 32.5 & 15 & " \\ 71 & 12 & 41 & 14 & 67 & 58.5 & 15 & " \\ 72 & 12 & 41 & 14 & 67 & 19.5 & 15 & " \\ 75 & 12 & 40 & 59 & 67 & 34 & 15 & " \\ 76 & 12 & 40 & 54.7 & 68 & 11 & 15 & " \\ 77 & 12 & 40 & 53 & 68 & 50.5 & 15 & . " \\ 78 & 12 & 40 & 58.3 & 69 & 38 & 16 & " \\ 79 & 12 & 40 & 58 & 70 & 10 & 16 & "\end{array}$

Lat.

Long.

Date

No Returns

" .

"

"

"

"

"

"

"

"

" 
Harengus Cruise 25

$15-30$ October

1958

All $8 \mathrm{oz}$, bottles, ballasted.

Released

Recovered

No.

Bottles

Sta.\# Released Bottle \# Lat. Long. Date Lat. Long. Date

95

94

12

93

92

91

90

89

88

87

8

8

\section{4}

83

82

35

8

\section{7}

98

99

100

101

12

12

12

12

12

12

12

12

12

12

12

12

12

12

12

12

12

12

12
12

61085

61088

61092

61095

$\begin{array}{lllllll}61001 & 44 & 57.8 & 66 & 51.2 & 15 & \text { Oct. } \\ 61003 & 44 & 57.8 & 66 & 51.2 & 15 & " \\ 61005 & 44 & 57.8 & 66 & 51.2 & 15 & " \\ 61008 & 44 & 57.8 & 66 & 51.2 & 15 & " \\ 61009 & 44 & 57.8 & 66 & 51.2 & 15 & " \\ & 44 & 31.2 & 67 & 12 & 15 & " \\ & 44 & 22.8 & 67 & 38 & 15 & " \\ & 44 & 00 & 67 & 38 & 15 & " \\ & 44 & 00 & 68 & 33.5 & 16 & " \\ & 43 & 40.2 & 68 & 33.5 & 16 & " \\ & 43 & 40.2 & 69 & 00 & 16 & " \\ 61085 & 43 & 22.4 & 70 & 21 & 16 & " \\ 61088 & 43 & 22.4 & 70 & 21 & 16 & " \\ 61092 & 43 & 22.4 & 70 & 21 & 16 & " \\ 61095 & 43 & 22.4 & 70 & 21 & 16 & " \\ & 43 & 00 & 69 & 55.2 & 16 & " \\ & 43 & 00 & 69 & 00 & 17 & " \\ & 43 & 22.4 & 69 & 00 & 17 & " \\ & 43 & 22.4 & 68 & 00 & 17 & " \\ & 43 & 40.2 & 68 & 00 & 17 & " \\ & 43 & 40.2 & 67 & 00 & 17 & " \\ & 43 & 51.8 & 66 & 45.5 & 17 & " \\ & 43 & 40.2 & 66 & 13.4 & 20 & " \\ 61202 & 43 & 24 & 66 & 30 & 20 & " \\ & 43 & 07.2 & 66 & 44.5 & 20 & " \\ & 42 & 50.5 & 66 & 58.5 & 20 & " \\ & 42 & 33.8 & 66 & 13.5 & 20 & " \\ & 42 & 25.5 & 67 & 20.5 & 20 & "\end{array}$

$\begin{array}{llllll}44 & 58.9 & 67 & 01.1 & 1 \text { Nov. '58 }\end{array}$

$\begin{array}{llllll}45 & 01.1 & 66 & 51 & 1\end{array}$

$\begin{array}{lllllll}44 & 53.6 & 67 & 05.2 & 21 & \text { Oct. '58 }\end{array}$



$\begin{array}{llllll}44 & 56 & 66 & 58 & 25 & \text { Oct. '58 }\end{array}$

No Returns

$\begin{array}{llllll}42 & 59 & 70 & 45 & 21 \mathrm{Mar} . & \\ 6 & 60\end{array}$

$\begin{array}{llllll}42 & 59 & 70 & 45.8 & 9 \text { Nov. '58 }\end{array}$

$\begin{array}{lllllll}42 & 59.6 & 70 & 45 & 30 & \text { Oct. '58 }\end{array}$

$\begin{array}{lllllll}42 & 53 & 70 & 49 & 29 & \text { Oct. '58 }\end{array}$

No Returns

$\begin{array}{llllll}43 & 59 & 66 & 09.5 & 27 & \text { Dec. '58 }\end{array}$ No Returns 
Harengus Cruise 25 (cont'd)

Released

Recovered

No.

Bottles

Sta.\# Released Bottle\# Lat. Long. Date Lat. Long. Date




Released

Recovered

No.

Bottles

Sta.\# Released Bottle \# Lat. Long. Date Lat. Long. Date

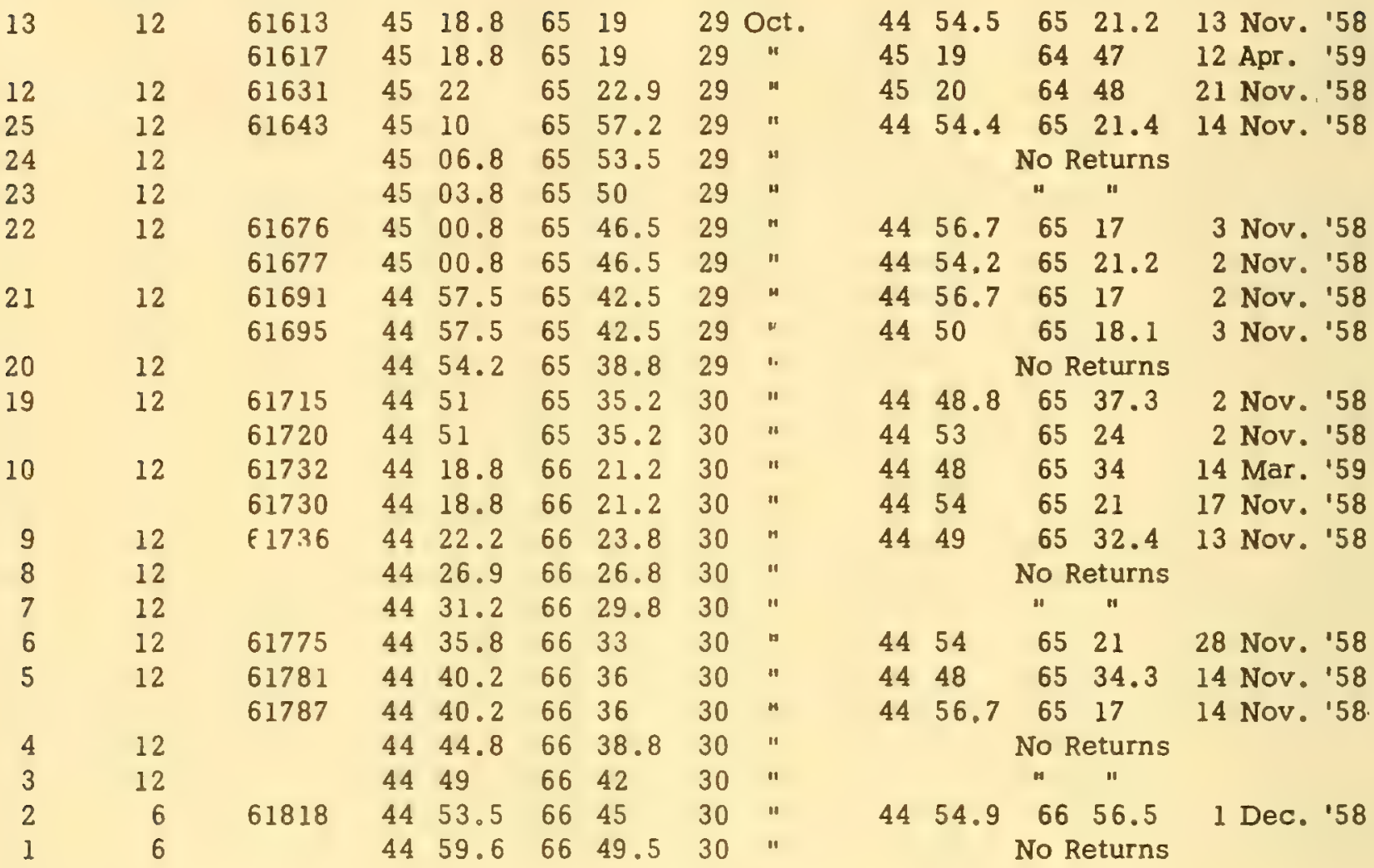


Albatross III Cruise 120

\section{$21-28$ October \\ 1958}

Odd numbers ballasted.

Released

Recovered

No.

Bottles

Sta.\# Released Bottle\# Lat. Long. Date Lat. Long. Date

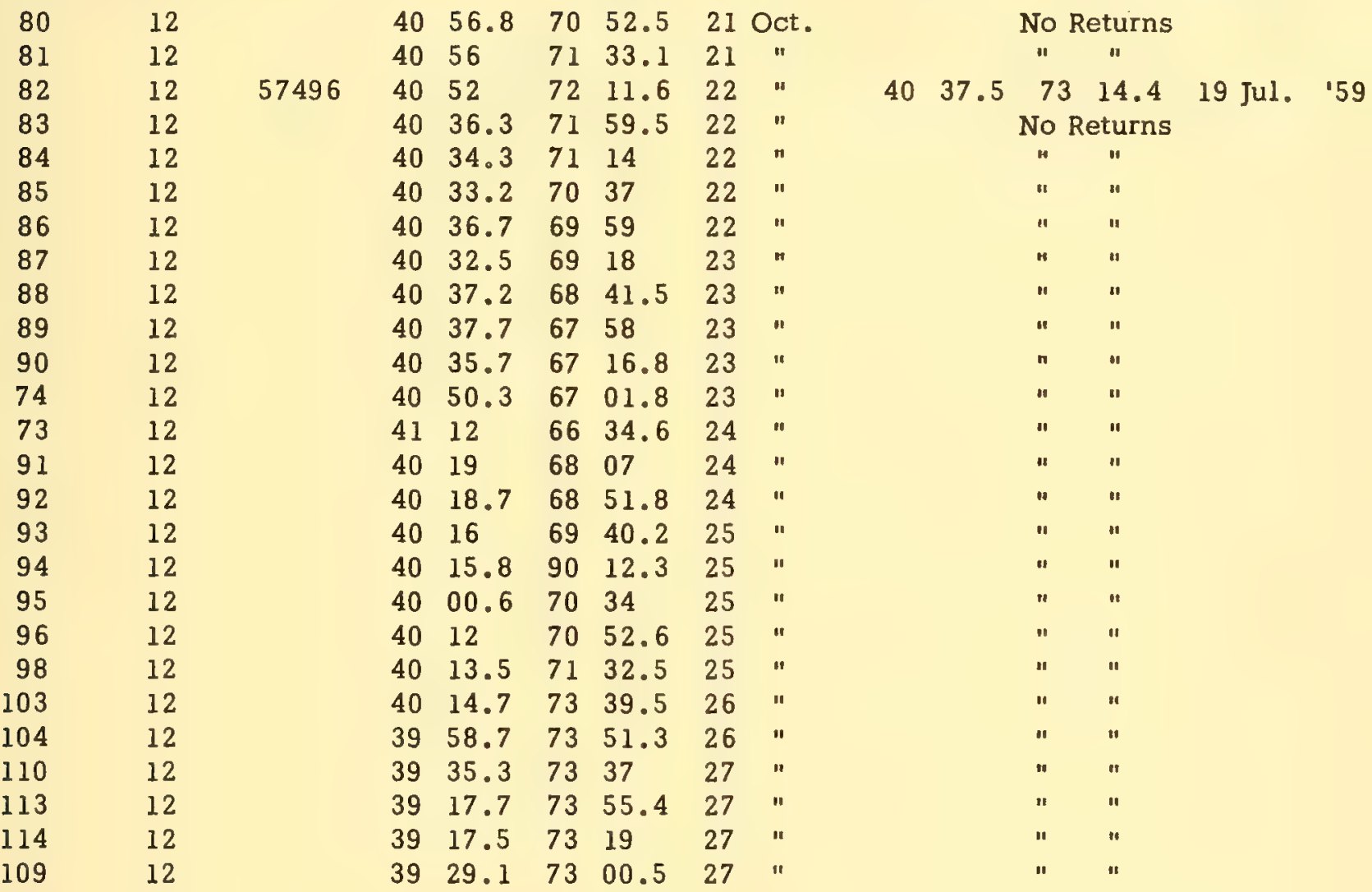


Albatross III Cruise 120 (cont'd)

Released

Recovered

No.

Bottles

Sta.\# Released

Bottle \#

Lat. Long. Date

Lat. Long D Date

$\begin{array}{rr}105 & 12 \\ 101 & 12 \\ 102 & 12 \\ 100 & 12 \\ 99 & 12 \\ 106 & 12 \\ 108 & 12 \\ 107 & 12 \\ 97 & 12\end{array}$

$\begin{array}{llllll}39 & 50.2 & 73 & 10 & 27 & \text { Oct. }\end{array}$

$\begin{array}{llllll}40 & 11 & 72 & 57 & 27\end{array}$

$\begin{array}{lllllll}40 & 28.8 & 73 & 12.1 & 27\end{array}$

$\begin{array}{llllll}40 & 31.3 & 72 & 39.5 & 27\end{array}$

$\begin{array}{llllll}40 & 13.6 & 72 & 18 & 28\end{array}$

$\begin{array}{lllllll}39 & 53.5 & 72 & 32.2 & 28\end{array}$

$\begin{array}{lllllll}39 & 36 & 72 & 18.1 & 28\end{array}$

$\begin{array}{lllllll}39 & 51.5 & 71 & 53.7 & 28\end{array}$

$\begin{array}{llllll}39 & 58.5 & 71 & 20 & 28\end{array}$

No Returns

" "

"

"

" 1

" 1

" "

"

" 
Harengus Cruise 27

10 - 24 November

1958

All $8 \mathrm{oz}$. bottles, weighted.

Released

Recovered

No.

Bottles

Sta.\# Released Bottle\# Lat. Long. Date Lat. Long. Date

\begin{tabular}{|c|c|c|c|c|c|c|c|c|c|c|c|c|c|}
\hline 95 & & 44 & 57.8 & 66 & 51.2 & 10 & Nov. & \multicolumn{6}{|c|}{ No Returns } \\
\hline 94 & 61849 & 44 & 31.2 & 67 & 12 & 10 & $"$ & 44 & 54.1 & 65 & $2 I^{\prime}$ & 21 May & 159 \\
\hline 93 & 61857 & 44 & 22.8 & 67 & 28 & 10 & $"$ & 44 & 23.8 & 66 & $12^{\prime}$ & 8 Jan. & '59 \\
\hline 92 & 61868 & 44 & 00 & 67 & 38 & 10 & $"$ & 44 & 11.5 & 66 & 10 & 3 Dec. & 158 \\
\hline 91 & & 44 & 00 & 68 & 33.5 & 11 & $"$ & \multicolumn{6}{|c|}{ No Returns } \\
\hline 90 & & 43 & 40.2 & 68 & 33.5 & 11 & $"$ & & & $"$ & $"$ & & \\
\hline 89 & & 43 & 40.2 & 69 & 00 & 11 & $"$ & & & $"$ & $"$ & & \\
\hline 88 & & 43 & 22.4 & 70 & 21 & 11 & $"$ & & & $"$ & $"$ & & \\
\hline 87 & & 43 & 00 & 69 & 55.2 & 11 & $"$ & & & $"$ & $"$ & & \\
\hline 86 & & 43 & 00 & 69 & 00 & 11 & $"$ & & & $"$ & $"$ & & \\
\hline 85 & & 43 & 22.4 & 69 & 00 & 12 & $"$ & & & $"$ & $"$ & & \\
\hline 84 & 61965 & 43 & 22.4 & 68 & 00 & 12 & $"$ & 43 & 39.5 & 65 & 49 & 20 Jan. & 159 \\
\hline & 61972 & 43 & 22.4 & 68 & 00 & 12 & $"$ & 44 & 00 & 66 & 09.3 & $18 \mathrm{Jan}$. & '59 \\
\hline 83 & & 43 & 40,2 & 68 & 00 & 12 & $"$ & \multicolumn{6}{|c|}{ No Returns } \\
\hline 82 & & 43 & 40.2 & 67 & 00 & 12 & $"$ & & & $"$ & $"$ & & \\
\hline 81 & 61999 & 43 & 40.2 & 66 & 13.6 & 14 & $"$ & 44 & 16 & 66 & 08 & 22 Nov. & '58 \\
\hline 97 & 62011 & 43 & 24 & 66 & 30 & 14 & $"$ & 44 & 22 & 66 & 05.5 & $14 \mathrm{Feb}$ & $' 59$ \\
\hline & 62016 & 43 & 24 & 66 & 30 & 14 & $"$ & 44 & 33 & 66 & 02 & 27 Dec. & 58 \\
\hline & 62020 & 43 & 24 & 66 & 30 & 14 & $"$ & 44 & 11.5 & 66 & 10 & 28 Nov. & 58 \\
\hline 12 & & 43 & 07.2 & 66 & 44.5 & 14 & $"$ & \multicolumn{6}{|c|}{ No Returns } \\
\hline 12 & & 42 & 50.5 & 66 & 58.5 & 14 & $"$ & & & $"$ & $"$ & & \\
\hline 12 & & 42 & 33.8 & 67 & 13.5 & 14 & $"$ & & & $"$ & " & & \\
\hline 12 & & 42 & 25.5 & 67 & 20.5 & 15 & $"$ & & & $"$ & $"$ & & \\
\hline 12 & & 42 & 17 & 67 & 27.5 & 15 & $"$ & & & $"$ & $"$ & & \\
\hline 12 & & 42 & 09 & 67 & 34.5 & 15 & $"$ & & & $"$ & $"$ & & \\
\hline 12 & & 42 & 04.2 & 67 & 23.2 & 15 & $"$ & & & $"$ & $"$ & & \\
\hline 12 & & 42 & 12 & 67 & 16.5 & 15 & $"$ & & & $"$ & $"$ & & \\
\hline
\end{tabular}


Released

Recovered

No.

Bottles

Sta.\# Released Bottle\# Lat. Long. Date Lat. Long. Date

\begin{tabular}{|c|c|c|c|c|c|c|c|c|c|c|c|c|c|c|}
\hline 106 & 12 & & 42 & 36.5 & 67 & 05 & 15 & Nov. & \multicolumn{6}{|c|}{ No Returns } \\
\hline 107 & 12 & & 42 & 15 & 66 & 57.2 & 15 & $"$ & & & $"$ & $"$ & & \\
\hline 108 & 12 & & 42 & 09.5 & 66 & 46.8 & 15 & $"$ & & & $"$ & $"$ & & \\
\hline 109 & 12 & & 42 & 04.5 & 66 & 35 & 15 & $n$ & & & $"$ & $"$ & & \\
\hline 110 & 12 & & 42 & 12.5 & 66 & 28 & 15 & $"$ & & & $"$ & $"$ & & \\
\hline 111 & 12 & & 42 & 22.8 & 66 & 25.5 & 15 & $"$ & & & $"$ & $"$ & & \\
\hline 112 & 12 & & 42 & 32.4 & 66 & 24 & 15 & $"$ & & & $"$ & $"$ & & \\
\hline 113 & 12 & & 42 & 42.2 & 66 & 22 & 15 & $"$ & & & " & $"$ & & \\
\hline 114 & 12 & & 43 & 02 & 66 & 18 & 15 & $"$ & & & $"$ & " & & \\
\hline 115 & 12 & 62230 & 43 & 21.5 & 66 & 14.5 & 15 & $"$ & 43 & 59 & 66 & 09.2 & 24 Nov. & $' 58$ \\
\hline & & 62233 & 43 & 21.5 & 66 & 14.5 & 15 & $"$ & 44 & 11.5 & 66 & 09.7 & 8 Dec. & $' 58$ \\
\hline & & 62234 & 43 & 21.5 & 66 & 14.5 & 15 & $"$ & 44 & 12 & 66 & 09.5 & 8 Dec. & 58 \\
\hline 81 & 12 & & 43 & 40.2 & 66 & 13.4 & 16 & $"$ & \multicolumn{6}{|c|}{ No Returns } \\
\hline 35 & 12 & 62252 & 43 & 51.8 & 66 & 45.5 & 18 & $"$ & 44 & 16.5 & 66 & 08.2 & 5 Dec. & $' 58$ \\
\hline & & 62257 & 43 & 51.8 & 66 & 45.5 & 18 & $"$ & 44 & 20 & 66 & 0.7 .5 & 25 Nov. & 158 \\
\hline & & 62258 & 43 & 51.8 & 66 & 45.5 & 18 & $"$ & 44 & 49 & 65 & 32.5 & 1 Dec. & '58 \\
\hline 34 & 12 & 62263 & 44 & 06.8 & 66 & 21.5 & 18 & $"$ & 44 & 20 & 66 & 07.5 & 14 Dec. & '58 \\
\hline & & 62264 & 44 & 06.8 & 66 & 21.5 & 18 & $"$ & 44 & 14.3 & 66 & 23 & 7 Dec。 & $' 58$ \\
\hline & & 62271 & 44 & 06.8 & 66 & 21.5 & 18 & $"$ & 44 & 20 & 66 & 07.5 & 24 Nov. & '58 \\
\hline 33 & 12 & & 44 & 18.1 & 66 & 42.8 & 18 & $"$ & \multicolumn{6}{|c|}{ No Returns } \\
\hline 32 & 12 & & 44 & 40.2 & 65 & 54.2 & 18 & $"$ & & & $"$ & $"$ & & \\
\hline 11 & 12 & & 44 & 43.2 & 65 & 58.8 & 18 & " & & & $"$ & $"$ & & \\
\hline 31 & 12 & 62316 & 45 & 46.5 & 66 & 03.8 & 18 & $"$ & 44 & 54.3 & 65 & 21.2 & 25 Nov. & \\
\hline 30 & 12 & & 44 & 49.2 & 66 & 08.3 & 19 & $"$ & \multicolumn{6}{|c|}{ No Returns } \\
\hline 29 & 12 & 62335 & 44 & 52.2 & 66 & 13 & 19 & $"$ & 44 & 54.5 & 65 & 21.3 & 10 Dec. & \\
\hline 27 & 12 & & 44 & 58.5 & 66 & 22.8 & 19 & $"$ & \multicolumn{6}{|c|}{ No Returns } \\
\hline 26 & 12 & 62357 & 45 & 01 & 66 & 26.8 & 19 & $"$ & 44 & 47 & 65 & 36.5 & 3 Dec. & '58 \\
\hline 25 & 12 & 62380 & 45 & 10 & 65 & 57.2 & 19 & $"$ & 45 & 00.5 & 65 & 09.2 & 25 Nov. & \\
\hline 24 & 12 & & 45 & 06.8 & 65 & 53.5 & 19 & " & \multicolumn{6}{|c|}{ No Returns } \\
\hline 23 & 12 & & 45 & 03.8 & 65 & 50 & 19 & $"$ & & & $"$ & $"$ & & \\
\hline 22 & 12 & & 45 & 00.8 & 65 & 46.5 & 19 & $"$ & & & $"$ & $"$ & & \\
\hline 21 & 12 & & 44 & 57.5 & 65 & 42.5 & 19 & " & & & $"$ & $"$ & & \\
\hline 20 & 12 & & 44 & 54.2 & 65 & 38.8 & 19 & $"$ & & & $"$ & " & & \\
\hline
\end{tabular}


Released

No.

Bottles

Sta.\# Released Bottle \# Lat. Long. Date Lat. Long. Date

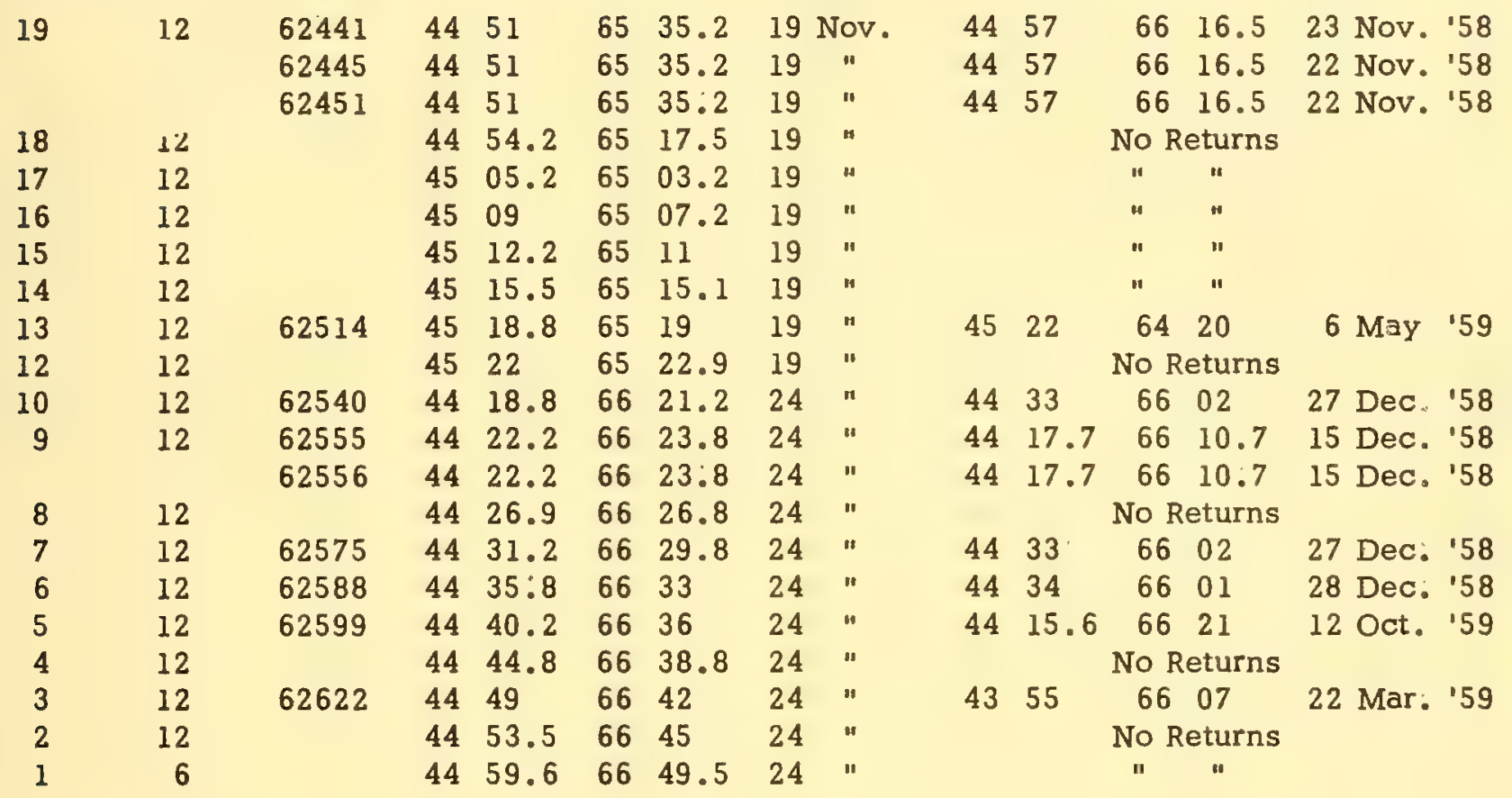


Delaware 58-7

12 - 19 December

1958

All bottles ballasted

Released

Recovered

No.

Bottles

Sta.\# Released Bottle \#

Lat. Long.

Date

Lat.

Long.

Date

$\begin{array}{rr}1 & 12 \\ 3 & 12 \\ 6 & 12 \\ 8 & 12 \\ 9 & 12 \\ 11 & 12 \\ 12 & 12\end{array}$

$41 \quad 42$

$69 \quad 10$

12 Dec.

4130

$\begin{array}{ll}69 & 15\end{array}$

12 n

$40 \quad 02$

$\begin{array}{ll}69 & 08\end{array}$

18 "

$40 \quad 10$

$\begin{array}{lll}68 & 12\end{array}$

18 "

$40 \quad 21$

$\begin{array}{ll}67 & 40\end{array}$

$18 "$

4128

$\begin{array}{ll}68 & 49\end{array}$

19 "

$42 \quad 05$

$\begin{array}{ll}69 & 57\end{array}$

19 "

No Returns


Sambro Lightship, $44^{\circ} 21.7^{\prime} \mathrm{N} \quad 63^{\circ} 24.3^{\prime} \mathrm{W}$

2 ballasted bottles launched each day at noon

commencing on 9 October 1958

\begin{tabular}{|c|c|c|c|c|c|}
\hline \multirow[b]{2}{*}{ Bottle \# } & \multirow[b]{2}{*}{ Release Date } & \multicolumn{4}{|c|}{ Recovery } \\
\hline & & Lat. & & ong. & Date \\
\hline 44658 & 15 Oct. & $44 \quad 33.8$ & 63 & 56 & 28 Oct. '58 \\
\hline 44676 & 24 Oct & $44 \quad 07.6$ & 64 & 31 & 29 Aug. '59 \\
\hline 44835 & 3 Dec. & $44 \quad 28$ & 63 & 42.6 & 12 Jun. '59 \\
\hline 14856 & 3 Dec. & $44 \quad 26.5$ & 63 & 46 & 10 Dec. '58 \\
\hline * & 31 Dec. & & & & \\
\hline
\end{tabular}

* No bottles released. 
Lurcher Lightship, $43^{\circ} 48.5^{\prime} \mathrm{N} \quad 66^{\circ} 31.8^{\prime} \mathrm{W}$

2 ballasted bottles launched each day at noon

commencing on 28 October 1956

Recovery

Bottle \# Release Date

Lat.

Long.

Date

15947

1956

15950

15996

8 Nov.

6 Dec.

11 "

$44 \quad 23$

$\begin{array}{ll}66 & 04\end{array}$

7 Dec. ' 56

16024

$17 "$

$\begin{array}{ll}65 & 45.5\end{array}$

12 Apr. 157

$44 \quad 37.5$

$\begin{array}{ll}66 & 02\end{array}$

20 Dec. ' 56

4348

$\begin{array}{ll}66 & 10\end{array}$

24 Dec. ' 56

\section{7}

16059

3 Jan.

16069

5 "

$44 \quad 27.5$

$\begin{array}{ll}66 & 00\end{array}$

24 Jan. ' 57

13 "

$44 \quad 22$

$\begin{array}{ll}66 & 05.5\end{array}$

24 Jan. 57

16072

14 "

$\begin{array}{ll}44 & 11.5\end{array}$

$\begin{array}{ll}66 & 10\end{array}$

4. Mar. 57

16095

16093

15 "

18 "

$\begin{array}{ll}66 & 08.8\end{array}$

24 Jan. 157

4411.5

$\begin{array}{ll}66 & 10\end{array}$

24 Jan. 157

16084

23 Jan. -2 Mar.

$44 \quad 23$

$66 \quad 05$

18 Mar. '57

28641

28622

4 Mar.

$44 \quad 17$

$\begin{array}{ll}66 & 08\end{array}$

26 Mar. '57

8 "

$44 \quad 15$

$\begin{array}{ll}66 & 08\end{array}$

26 Mar。 57

15 "

$66 \quad 10$

5 Apr. 157

28651

16 "

$\begin{array}{ll}66 & 19\end{array}$

5 Apr. '57

28671

28687

2 - 4 Apr.

$10 \mathrm{Apr}$.

18 "

28693

28694

28697

20 "

$21 "$

23 "

28704

28711

28714

28719

28717

26

30 "

1 May

$44 \quad 18$

6620

30 Apr. 57

$44 \quad 14$

$66 \quad 06.1$

30 Apr. $\quad 57$

$\begin{array}{ll}44 & 29.8\end{array}$

$\begin{array}{ll}64 & 27\end{array}$

29 Apr. 157

4421.6

$\begin{array}{ll}66 & 06.3\end{array}$

8 Jul. ' 57

$45 \quad 17$

6424

14 Nov. '57

$\begin{array}{ll}45 & 22.3\end{array}$

$\begin{array}{ll}64 & 25.5\end{array}$

8 Dec. '57

$45 \quad 19$

$\begin{array}{ll}64 & 27\end{array}$

29 Sept. 157

$44 \quad 36.6$

$\begin{array}{ll}65 & 55.3\end{array}$

$8 \mathrm{Feb}$. '58

2 "

$\begin{array}{ll}45 & 07.8\end{array}$

$\begin{array}{ll}67 & 02.5\end{array}$

21 Jul. 57

4 "

6456

3 Nov. '57

28718

4 "

$64 \quad 34$

6 Oct. ' 57

* No bottles released. 


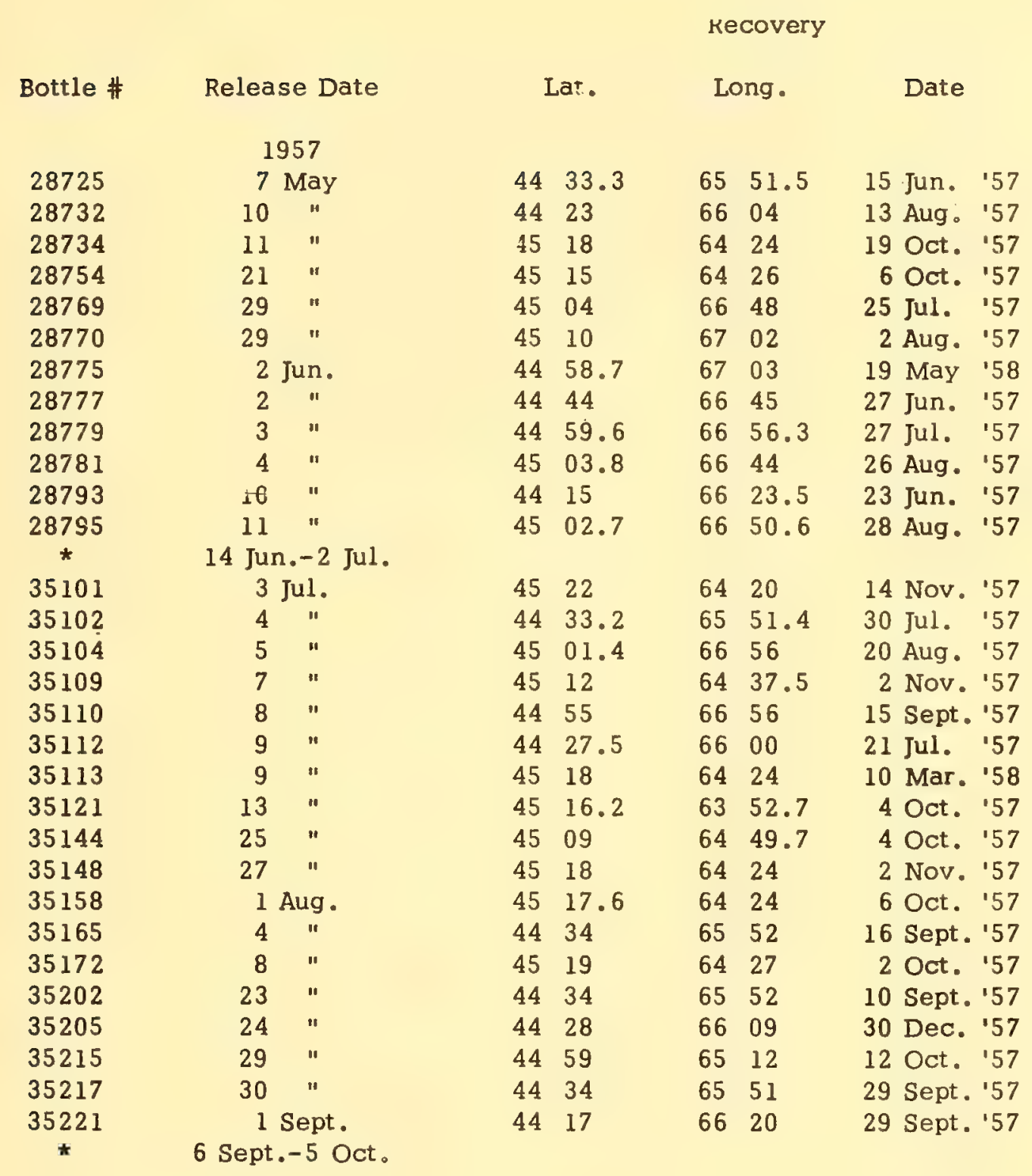

* No bottles released. 
Recovery

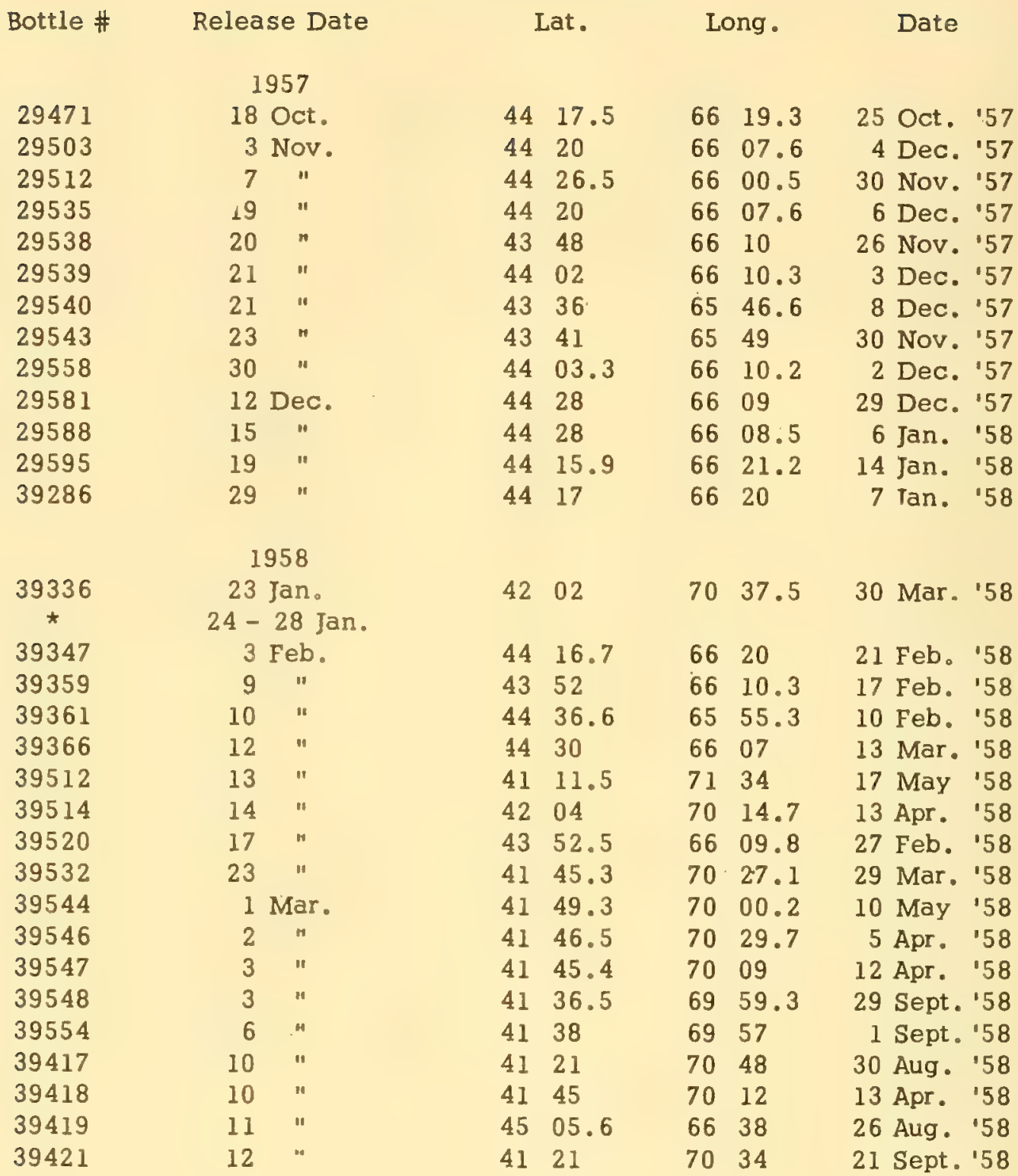

* No bottles released. 
Recovery

\begin{tabular}{|c|c|c|c|c|c|c|c|}
\hline \multirow{2}{*}{$\begin{array}{l}\text { Bottle \# } \\
39422\end{array}$} & \multirow{2}{*}{$\begin{array}{c}\text { Release Date } \\
1958 \\
12 \text { Mar. }\end{array}$} & \multicolumn{2}{|c|}{ Lat. } & \multicolumn{2}{|c|}{ Long. } & \multicolumn{2}{|l|}{ Date } \\
\hline & & 42 & 28 & 70 & 55.4 & 30 Apr. & '58 \\
\hline 39424 & $13 "$ & 41 & 46 & 70 & 05 & 13 Apr. & '58 \\
\hline 39443 & 23 & 42 & 41 & 70 & 45 & $10 \mathrm{May}$ & '58 \\
\hline 39449 & 26 & 44 & 57.2 & 66 & 54.4 & 20 Sept. & $: 58$ \\
\hline 39461 & 1 Apr. & 42 & 41.3 & 70 & 46.1 & 29 Jun. & 158 \\
\hline 39467 & 4 " & 44 & 20 & 66 & 07.6 & 3 May & '58 \\
\hline 39470 & 5 & 45 & 18.4 & 64 & 47.3 & 18 Jun. & $' 58$ \\
\hline 39472 & 6 & 44 & 28.5 & 67 & 40.5 & 27 May & '58 \\
\hline 39447 & $9 N$ & 45 & 10.2 & 64 & 44.7 & 3 Sept. & '58 \\
\hline$*$ & $13-25$ Apr. & & & & & & \\
\hline 39486 & 26 Apr. & 44 & 37 & 66 & 52 & 20 May & '58 \\
\hline 39491 & 29 & 44 & 38 & 66 & 43 & 11 May & '58 \\
\hline 39496 & 1 May & 45 & 02.5 & 66 & 50.8 & $29 \mathrm{Jul}$. & '58 \\
\hline 39499 & $"$ & 44 & 48.5 & 66 & 58.7 & 30 May & '58 \\
\hline 39373 & 12 & 44 & 23 & 66 & 04 & 3 Sept. & '58 \\
\hline 39377 & 14 & 44 & 42 & 66 & 44 & 5 Jun. & '58 \\
\hline 39383 & 17 & 45 & 00.2 & 66 & 56.8 & 6 Jun. & 158 \\
\hline 39388 & 19 & 44 & 48.6 & 66 & 58.7 & 27 Aug. & '58 \\
\hline $\begin{array}{c}39414 \\
*\end{array}$ & $\begin{array}{l}1 \text { Jun. } \\
2-7 \text { Jun. }\end{array}$ & 45 & 18 & 64 & 2.4 & 4 Apr. & '59 \\
\hline 51625 & 15 Jun. & 44 & 54 & 65 & 20 & 20 May & 159 \\
\hline 51640 & 23 & 44 & 53 & 66 & 57.2 & 2 Nov. & '58 \\
\hline 51649 & 28 & 45 & 23 & 64 & 19 & 17 Jan. & '59 \\
\hline 51652 & 29 & 44 & 48.6 & 66 & 58.8 & $31 \mathrm{Jul}$. & 158 \\
\hline 51658 & 2 Jul. & 45 & 00.2 & 66 & 55.9 & 24 Sept. & '58 \\
\hline 51691 & $19 \quad x$ & 45 & 18 & 64 & 24 & 27 Sept. & '58 \\
\hline 51710 & 28 & 45 & 00.5 & 65 & 08.5 & 6 Oct. & '58 \\
\hline 51711 & 29 & 44 & 55.6 & 67 & 01 & 20 Sept. & '58 \\
\hline 51727 & 6 Aug. & 44 & 23 & 66 & 04 & 19 Sept. & '58 \\
\hline 51761 & $23 \quad 1$ & 45 & 02.4 & 66 & 53.5 & 12 Oct. & '58 \\
\hline $\begin{array}{c}51783 \\
*\end{array}$ & $\begin{array}{c}3 \text { Sept. } \\
5 \text { Sept.-28 Cat. }\end{array}$ & 44 & 20 & 66 & 07.5 & 13 Sept. & '58 \\
\hline 51898 & 7 Nov. & 44 & 48.8 & 65 & 32.5 & 27 Nov. & '58 \\
\hline 51921 & $19 \quad "$ & 43 & 48 & 66 & 09.5 & 3 Mar. & 159 \\
\hline
\end{tabular}

* No bottles released. 


\section{Recovery}

\begin{tabular}{|c|c|c|c|c|}
\hline Bottle \# & $\begin{array}{c}\text { Release Date } \\
1958\end{array}$ & Lat. & Long. & Date \\
\hline 51937 & 23 Nov. & 4430 & $\begin{array}{ll}66 & 06\end{array}$ & 1 Dec. \\
\hline 51965 & 9 Dec. & $44 \quad 33$ & $\begin{array}{ll}66 & 02\end{array}$ & 27 Dec. \\
\hline 51967 & 10 & $43 \quad 59.2$ & $\begin{array}{ll}66 & 09.3\end{array}$ & 22 Dec. \\
\hline 51971 & 12 & $43 \quad 59.2$ & $\begin{array}{ll}66 & 09.3\end{array}$ & 22 Dec. \\
\hline 51986 & 19 & $43 \quad 26.5$ & $\begin{array}{ll}65 & 38\end{array}$ & $4 \mathrm{May}$ \\
\hline 51999 & 26 & $43 \quad 42.4$ & $65 \quad 50$ & 10 Jun. \\
\hline
\end{tabular}


Princess Helene

2 ballasted bottles released each day at

$45^{\circ} 07^{\prime} \mathrm{N}, 65^{\circ} 59^{\prime} \mathrm{W}$

beginning 18 December 1958

Recovery

Bottle \#

Release Date

Lat.

Long .

Date

1958

$21 \mathrm{Dec}$.

50448

26 "

4423.7

$66 \quad 12$

10 Jan. '59

50449

$27 "$

$28 "$

4433

6602

10 Feb. '59 
Portland Lightship

\section{$43^{\circ} 32^{\prime} \mathrm{N} \quad 70^{\circ} 06^{\prime} \mathrm{W}$}

2 ballasted bottles released each day

beginning 23 July 1958

\section{Recovery}

\begin{tabular}{|c|c|c|c|c|c|c|c|}
\hline Bottle \# & Release Date & & at. & & ng. & Date & \\
\hline & 1958 & & & & & & \\
\hline 55388 & $24 \mathrm{Jul}$. & 43 & 41 & 70 & 10.4 & 31 Jul. & 158 \\
\hline 55368 & $25 \quad "$ & 43 & 31.6 & 70 & 18.9 & '27 Jul. & 158 \\
\hline 55369 & 25 & 43 & 33.5 & 70 & 17 & 29 Jul. & 158 \\
\hline 55378 & $26 \quad "$ & 43 & 30 & 70 & 23 & $27 \mathrm{Jul}$. & 158 \\
\hline 55367 & $26 \quad " 1$ & 43 & 31 & 70 & 22.5 & $27 \mathrm{Jul}$. & '58 \\
\hline 55380 & Aug. & 43 & 44.4 & 70 & 11 & 7 Aug. & '58 \\
\hline 55389 & $1 "$ & 43 & 44.3 & 70 & 00 & 6 Aug. & $' 58$ \\
\hline 55409 & 10 & 43 & 42.1 & 69 & 50.0 & 22 Aug. & 158 \\
\hline 55397 & $11 "$ & 43 & 45.5 & 69 & 58.4 & 19 Aug. & 158 \\
\hline 55401 & $12 \quad "$ & 43 & 46.5 & 69 & 59.5 & 23 Aug. & 158 \\
\hline 55126 & 15 & 43 & 33.2 & 70 & 14.6 & 22 Aug. & $' 58$ \\
\hline 55128 & $16 \quad$ & 42 & 00 & 70 & 05 & 17 May & '59 \\
\hline 55139 & 19 & 43 & 33.2 & 70 & 14.6 & I Sept. & .58 \\
\hline 55138 & 19 & 43 & 35.2 & 70 & 12.3 & 30 Oct. & 158 \\
\hline 55137 & 21 & 43 & 32.5 & 70 & 19.5 & 3 I Aug. & 158 \\
\hline 55142 & 23 & 43 & 33.2 & 70 & 14.6 & 1 Sept. & 158 \\
\hline 55141 & 25 & 43 & 45 & 70 & 03.3 & 31 Aug. & '58 \\
\hline 55143 & 25 & 43 & 45 & 69 & 47 & 8 May & 159 \\
\hline 55165 & 28 & 43 & 46.7 & 70 & 01 & 12 Nov. & $' 58$ \\
\hline 55167 & 28 & 43 & 49 & 69 & 58 & 20 Jun. & '59 \\
\hline 55156 & 30 & 43 & 44 & 69 & 51.2 & 5 Sept. & 158 \\
\hline 55164 & 3 Sept. & 43 & 47 & 69 & 54 & 14 Sept. & .58 \\
\hline 55146 & 311 & 43 & 39.4 & 70 & 02.2 & 16 Oct. & '58 \\
\hline 53395 & 23 & 42 & 01.7 & 70 & 37.5 & 19 Oct. & '58 \\
\hline 55432 & 6 Oct. & 42 & 41 & 70 & 44.7 & 26 Oct. & ' 58 \\
\hline 55417 & 22 & 43 & 10.5 & 70 & 36.4 & 2 Nov. & '58 \\
\hline
\end{tabular}

9 Nov. -18 Dec.

* No bottles released. 
Texas Tower \#2

$$
41^{\circ} 41^{\prime} \mathrm{N} \quad 67^{\circ} 45^{\prime} \mathrm{W}
$$

2 ballasted bottles released near noon each day

commencing on 31 January 1958 and continuing through 31 December 1958

$$
\text { Recovery }
$$

\begin{tabular}{|c|c|c|c|c|}
\hline Bottle \# & Release Date & Lat. & Long. & Date \\
\hline $\begin{array}{l}44325 \\
* \\
*\end{array}$ & $\begin{array}{l}4 \text { Mar. } \\
5 \text { - } 21 \text { Mar. } \\
25 \text { Apr. }\end{array}$ & $41 \quad 39$ & $70 \quad 12$ & 7 Sept. ${ }^{\prime} 58$ \\
\hline $\begin{array}{l}44398 \\
* \\
*\end{array}$ & $\begin{array}{l}24 \text { Jun. } \\
30 \text { " } \\
21,22 \text { Sept. }\end{array}$ & 4150 & $67 \quad 23$ & $31 \mathrm{Jan} .159^{* *}$ \\
\hline
\end{tabular}

* No bottles released.

** Apparently over ballasted, picked up in 31-32 fathoms of water. 
Nantucket Lightship

$$
40^{\circ} 33^{\prime} \mathrm{N} \quad 69^{\circ} 28^{\prime} \mathrm{W}
$$

2 ballasted bottles released each day

commencing on 28 July 1958

\section{Recovery}

\begin{tabular}{lcrrrrr} 
Bottle \# & Release Date & \multicolumn{2}{c}{ Lat. } & \multicolumn{2}{c}{ Long. } & \multicolumn{2}{c}{ Date } \\
& & & & & & \\
53171 & 17 Aug. & 41 & 25.3 & 70 & 54.6 & 7 Sept.'58 \\
53172 & 17 " & 41 & 25.3 & 70 & 54.6 & 7 Sept.'58 \\
53242 & 28 & 41 & 15.5 & 70 & 13.5 & 11 Sept.'58
\end{tabular}

* $\quad 15,20,30$ Oct.

* $\quad 15,16,26 \mathrm{Nov},-3 \mathrm{Dec}$.

* $\quad 6,7,24,25,28-31 \mathrm{Dec}$.

* No bottles released. 
Texas Tower \#4

$39^{\circ} 48^{\prime} \mathrm{N} \quad 72^{\circ} 40^{\prime} \mathrm{W}$

2 ballasted bottles released near noon each day commencing on 7 October 1958 and continuing through 31 December 1958 except for 4, 5, 9, 25 - 30 November and 1 - 6 December.

No Returns 


\section{Appendix}

Returns from Albatross III cruises of 1953, 1955, and 1956 received since 22 October 1957 , subsequent to returns published in S.S.R. No. 242.

Released

Sta.\# Bottle \#

$43 \quad 13421 \mathrm{~B}$

Lat. Long.

Date

Albatross III, Cruise 48

$41 \quad 44.2 \quad 69 \quad 50.2 \quad 30$ Apr. '53

$\begin{array}{llllll}41 & 11 & 69 & 15 & 1\end{array}$ $\begin{array}{llll}41 & 41 & 69 & 56\end{array}$

$\begin{array}{llll}41 & 05 & 70 & 40\end{array}$
Recovered

Lat. Long.

Date

Albatross III, Cruise 58



\section{Albatross III, Cruise 60}

\begin{tabular}{|c|c|}
\hline 2 & $17597 \mathrm{~B}$ \\
\hline 25 & $17887 \mathrm{~B}$ \\
\hline 30 & $17945 \mathrm{~B}$ \\
\hline 34 & 17988 \\
\hline 36 & 18011 \\
\hline 37 & 18027 \\
\hline
\end{tabular}

$\begin{array}{lllllll}41 & 00.5 & 71 & 01.5 & 19 \text { Apr. '55 } \\ 43 & 01.5 & 70 & 21 & \text { 22 Apr. '55 } \\ 42 & 52.5 & 67 & 05 & 23 \text { Apr. } 55 \\ 42 & 39.2 & 64 & 57 & 23 \text { Apr. '55 } \\ 42 & 16 & 65 & 31 & \text { 23 Apr. '55 } \\ 42 & 15.5 & 65 & 59.3 & \text { 23 Apr. '55 }\end{array}$

$\begin{array}{llll}35 & 24 & 75 & 29\end{array}$

5 Dec. ' 59

Albatross III, Cruise 61

$24 \quad 19092 \mathrm{~N}$

$42 \quad 33$

6438.8

21 May '55

$40 \quad 56$

$72 \quad 14$

30 Mar. '58

* Bottle recovered from bottom. 
Lat. Long. Date

Lat.

Long .

Date

\section{Albatross III, Cruise 71}

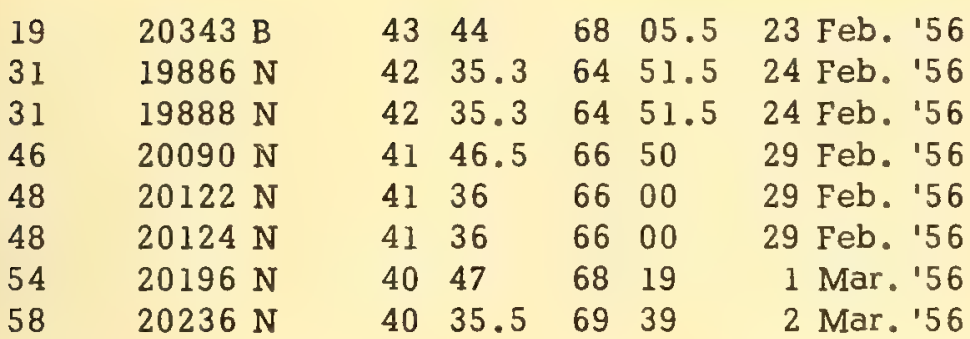

$\begin{array}{llllll}44 & 35 & 01 & 15 & 7 & \text { Mar. '59 }\end{array}$

$\begin{array}{llll}50 & 44 & 00 & 13 \mathrm{E}\end{array} 7 \mathrm{Nov}$. '57

$\begin{array}{llllll}41 & 28.7 & 70 & 45.5 & 30 \mathrm{Mar} \cdot{ }^{\prime 58}\end{array}$

\begin{tabular}{llllll}
49 & 32 & 01 & 55 & 27 & Dec. \\
\hline
\end{tabular}

$\begin{array}{llllll}44 & 30 & 01 & 14 & 9 \mathrm{Jan} . \text { '58 }\end{array}$

$\begin{array}{llllllll}56 & 45 & 08 & 15 & \mathrm{E} & 28 & \text { Oct. ' } 57\end{array}$

$\begin{array}{llllll}48 & 50 & 03 & 18 & 12 \mathrm{Jan} . & \end{array}$

$\begin{array}{lllll}65 & 41.7 & 12 & 26.3 \mathrm{E} & 16 \\ \mathrm{Nov} . & & 57\end{array}$

\section{Albatross III, Cruise 72}






\section{Albatross III, Cruise 73}

\begin{tabular}{|c|c|c|c|c|c|c|c|c|c|c|}
\hline 20973 B & 40 & 31.4 & 71 & 00.2 & 17 Apr. '56 & 44 & 08 & 01 & 15 & 12 Jan. \\
\hline 20991 B & 40 & 01.5 & 69 & 53.8 & 18 Apr. 156 & 52 & 05 & 10 & 00 & 21 Jan. \\
\hline $21023 \mathrm{~B}$ & 40 & 00 & 68 & 00 & 18 Apr. '56 & 47 & 54 & 04 & $00 \mathrm{~W}$ & $22 \mathrm{Mar}$. \\
\hline $21024 \mathrm{~N}$ & 40 & 00 & 68 & 00 & 18 Apr. 156 & 62 & 40 & 06 & $18 \mathrm{E}$ & 23 Dec. \\
\hline $21081 \mathrm{~B}$ & 43 & 22.2 & 66 & 20.4 & $19 \mathrm{Apr} .156$ & 45 & 53.2 & 64 & 32.7 & 4 Oct. \\
\hline $21126 \mathrm{~N}$ & 43 & 40.5 & 69 & 00 & 19 Apr. 156 & 41 & 51 & 69 & 57 & 17 May \\
\hline $21187 \mathrm{~B}$ & 42 & 48.5 & 68 & 03.0 & $20 \mathrm{Apr} . \quad 56$ & 58 & 20 & 11 & $20 E$ & 19 Oct. \\
\hline $21235 \mathrm{~B}$ & 42 & 01 & 65 & 12 & 21 Apr. '56 & 55 & 27 & 05 & 42 & 26 Jan. \\
\hline $21240 \mathrm{~N}$ & 42 & 01 & 65 & 12 & $21 \mathrm{Apr} . \quad 156$ & 55 & 33 & 08 & $05 \mathrm{E}$ & 21 Oct. \\
\hline $21249 \mathrm{~B}$ & 42 & 01.5 & 66 & 02 & $21 \mathrm{Apr} .156$ & 56 & 30 & 06 & 12 & 4 Jan. \\
\hline $21373 \mathrm{~B}$ & 41 & 30 & 66 & 54 & 23 Apr. '56 & 53 & 39 & 09 & 58 & 30 Oct. \\
\hline $21494 \mathrm{~N}$ & 42 & 13.2 & 65 & 46.8 & 25 Apr. 156 & 55 & 28 & 08 & $25 E$ & 30 Oct. \\
\hline $21495 \mathrm{~B}$ & 42 & 13.2 & 65 & 46.8 & 25 Apr. $\quad 56$ & 52 & 46 & 04 & 47 & 31 Jul. \\
\hline $21525 \mathrm{~B}$ & 42 & 59.5 & 65 & 10.1 & 26 Apr. '56 & 47 & & 04 & & $23 \mathrm{Feb}$ \\
\hline $21559 \mathrm{~B}$ & 42 & 19.8 & 66 & 56.7 & 26 Apr. '56 & 44 & 30 & 01 & 17 & 17 Aug. \\
\hline $21684 \mathrm{~N}$ & 40 & 44.5 & 68 & 08 & $28 \mathrm{Apr} .156$ & 36 & 42 & 75 & 55.5 & 11 \\
\hline $21712 \mathrm{~N}$ & 41 & 04.4 & 70 & 34.7 & $28 \mathrm{Apr} \cdot{ }^{\prime} 56$ & 41 & 39.6 & 70 & 00.7 & 28 \\
\hline
\end{tabular}

\section{Albatross III, Cruise 75}

121737 B

$321762 \mathrm{~N}$

$421771 \mathrm{~B}$

$421776 \mathrm{~N}$

$14 \quad 21891 \mathrm{~B}$

$1421894 \mathrm{~N}$

$1921952 \mathrm{~N}$

$1921960 \mathrm{~N}$

$32 \quad 22107 \mathrm{~B}$

$33 \quad 22123 \mathrm{~B}$

$47 \quad 22293$ B

$7022568 \mathrm{~N}$

$7122584 \mathrm{~N}$

$7222596 \mathrm{~N}$

$\begin{array}{lllllll}41 & 01.7 & 70 & 57.7 & 16 \text { May '56 } \\ 40 & 00.5 & 71 & 00 & 16 & \text { May '56 } \\ 39 & 57.8 & 69 & 33 & 17 \text { May '56 } \\ 39 & 58.7 & 69 & 33 & 17 \text { May '56 } \\ 43 & 17 & 66 & 43 & 18 \text { May '56 } \\ 43 & 17 & 66 & 43 & 18 \text { May '56 } \\ 44 & 15.5 & 66 & 55 & 19 \text { May '56 } \\ 44 & 15.5 & 66 & 55 & 19 \text { May '56 } \\ 43 & 40 & 68 & 58 & \text { 20 May '56 } \\ 43 & 32.4 & 70 & 01.9 & \text { 20 May '56 } \\ 41 & 59.2 & 65 & 04.2 & 23 \text { May '56 } \\ 40 & 40.6 & 67 & 55 & \text { 26 May '56 } \\ 40 & 37.2 & 67.24 & \text { 26 May '56 } \\ 40 & 38.5 & 66 & 46.6 & \text { 26 May '56 }\end{array}$

$\begin{array}{llll}41 & 24.3 & 70 & 31\end{array}$

$\begin{array}{lllll}47 & 48 & 04 & 20\end{array}$

22 Feb. ' 60

$\begin{array}{llll}39 & 00 & 32 & 50\end{array}$

$11 \mathrm{Feb}$. '58

6530

1215

$21 \mathrm{Jul.} \quad 158$

$\begin{array}{llllll}41 & 52.5 & 70 & 00.6 & 2 \text { Aug. } 58\end{array}$

$\begin{array}{lllll}41 & 46 & 69 & 56 & 26 \mathrm{Jan} . ' 58\end{array}$

$\begin{array}{llllll}42 & 03.5 & 70 & 14.7 & 9 & \text { Mar. '58 }\end{array}$

$\begin{array}{lllll}44 & 05.7 & 66 & 12.5 & 8 \mathrm{Jan} .\end{array}$

39

28

$14 \mathrm{Feb}$ ' 60

$\begin{array}{llllll}42 & 03.6 & 70 & 14.7 & 21 \text { May } & 60\end{array}$

$\begin{array}{llllll}52 & 16 & 10 & 04 & 3 \text { Nov. '57 }\end{array}$

\begin{tabular}{llllll}
38 & 02.0 & 75 & 20.3 & 30 \\
\hline
\end{tabular}

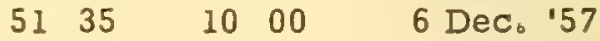

48

$23 \mathrm{Feb} . ' 58$ 
Lat. Long. Date Lat. Long.

Date

\section{Albatross III, Cruise 76}

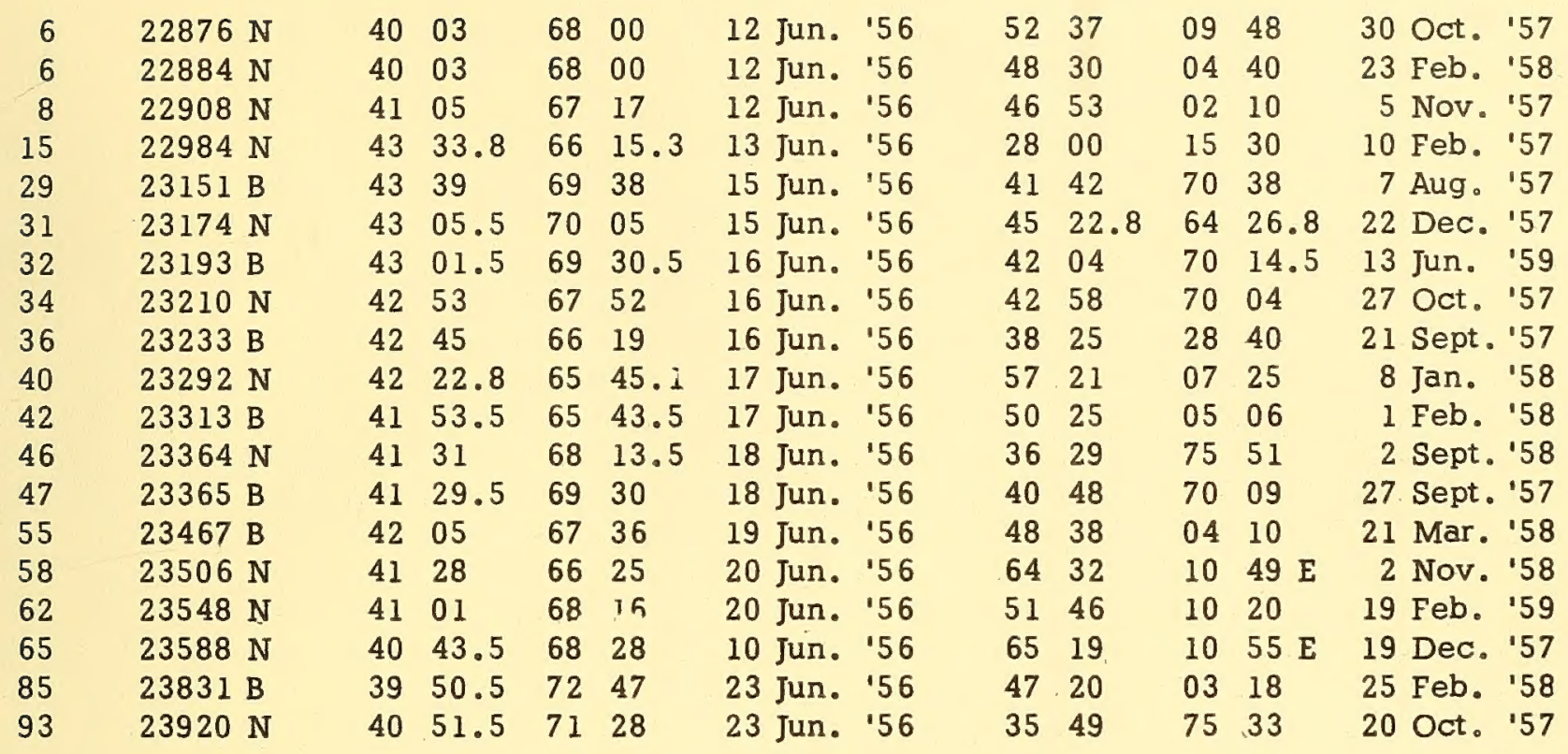

T. N. Gill, Cruise 8






